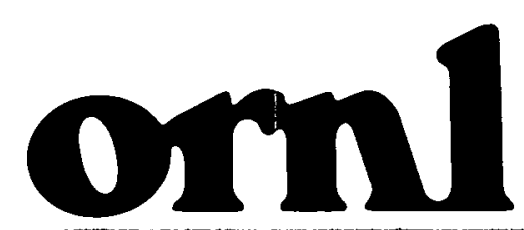

OAK RIDGE NATIONAL LABORATORY

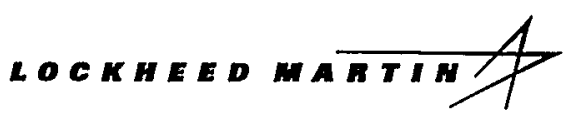

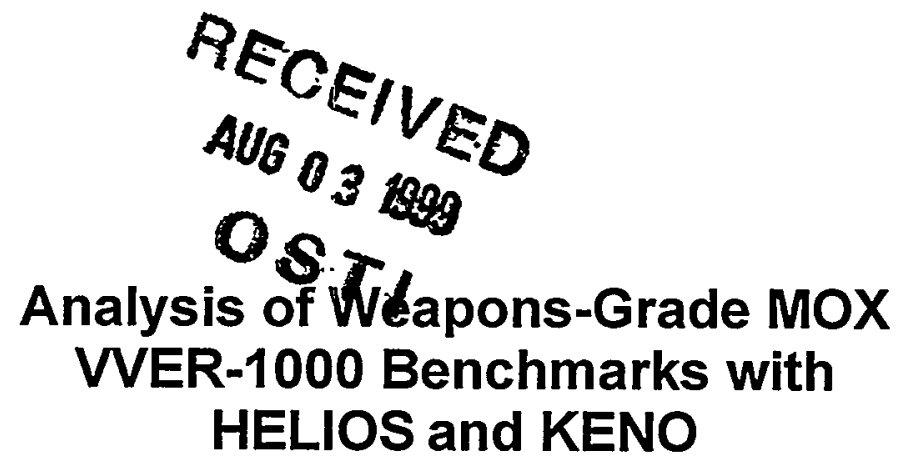

J. C. Gehin

C. Dourougie

M. B. Emmett

R. A. Lillie

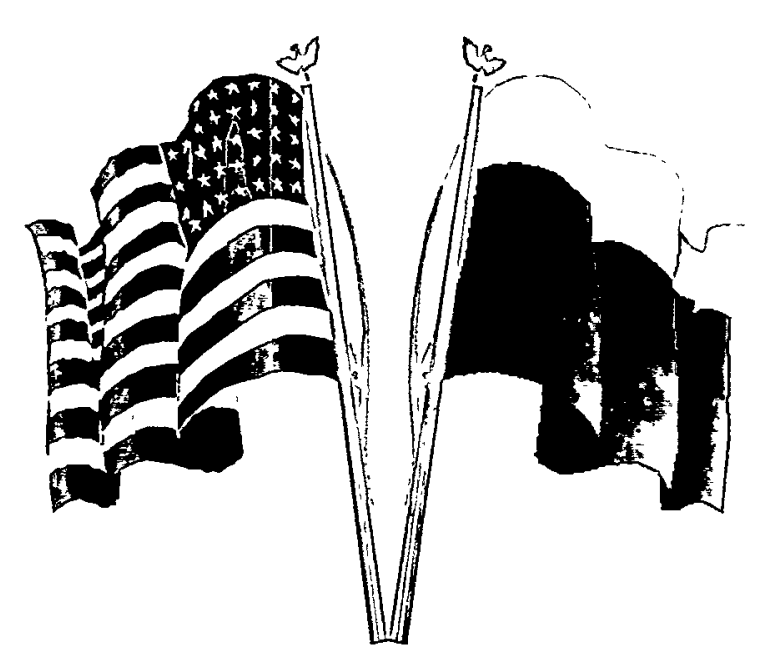

Fissile Materials Disposition Program
MANAGED AND OPERATED BY

LOCKHEED MARTIN ENERGY RESEARCH CORPORATION FOR THE UNTED STATES

DEPARTMENT OF ENERGY 
This report has been reproduced from the best available copy.

Reports are available to the public from the following source.

National Technical Information Service

5285 Port Royal Road

Springfield, VA 22161

Telephone 703-605-6000 (1-800-553-6847)

TDD 703-487-4639

Fax 703-605-6900

E-mail orders@ntis.fedworld.gov

Web site http://www.ntis.gov/ordering.htm

Reports are available to U.S. Department of Energy (DOE) employees, DOE contractors, Energy Technology Data Exchange (ETDE) representatives, and International Nuclear Information System (INIS) representatives from the following source.

Office of Scientific and Technical Information

P.O. Box 62

Oak Ridge, TN 37831

Telephone 423-576-8401

Fax 423-576-5728

E-mail reports@adonis.osti.gov

Web site http://www.osti.gov/products/sources.html

Reports produced after January 1,1996, are generally available via the DOE Information Bridge.

Web'site http://www.doe.gov/bridge

This report was prepared as an account of work sponsored by an agency of the United States Government. Neither the United States Government nor any agency thereof, nor any of their employees, makes any warranty, express or implied, or assumes any legal liability or responsibility for the accuracy, completeness, or usefulness or any information, apparatus, product, or process disclosed, or represents that its use would not infringe privately owned rights. Reference herein to any specific commercial product, process, or service by trade name, trademark, manufacturer, or otherwise, does not necessarily constitute or imply its endorsement, recommendation, or favoring by the United States Government or any agency thereof. The views and opinions of authors expressed herein do not necessarily state or reflect those of the United States Government or any agency thereof. 


\section{DISCLAIMER}

Portions of this document may be illegible in electronic image products. Images are produced from the best available original document. 
Computational Physics and Engineering Division

\title{
ANALYSIS OF WEAPONS-GRADE MOX VVER-1000 BENCHMARKS WITH HELIOS AND KENO
}

\author{
J. C. Gehin \\ C. Dourougie \\ M. B. Emmett \\ R. A. Lillie
}

July 1999

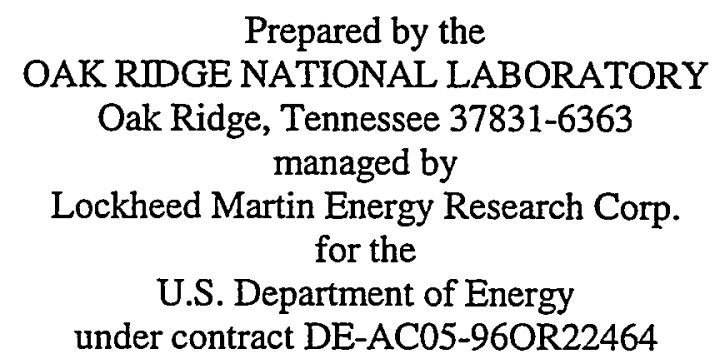


I 


\section{CONTENTS}

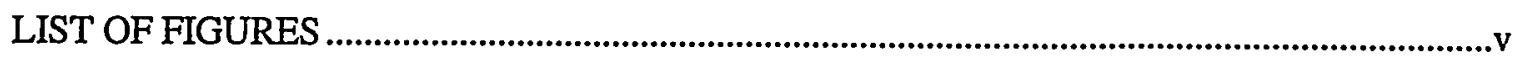

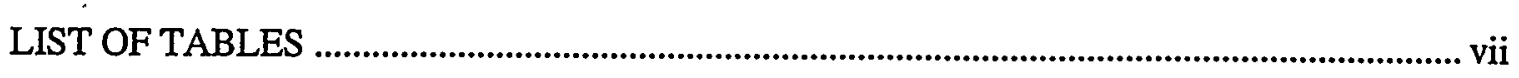

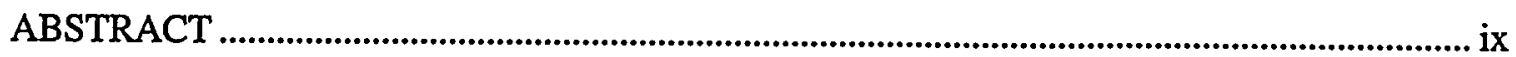

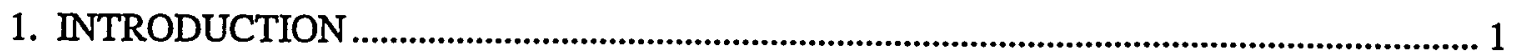

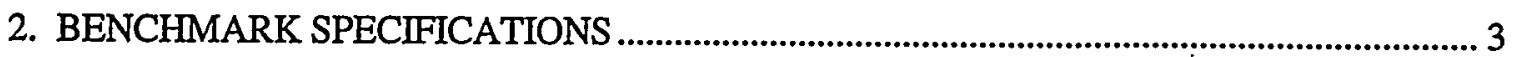

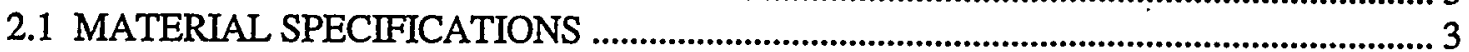

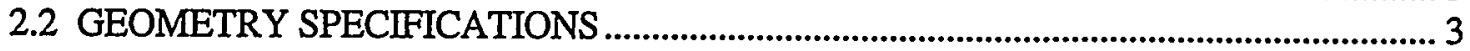

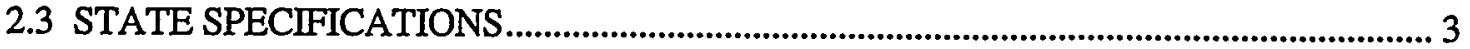

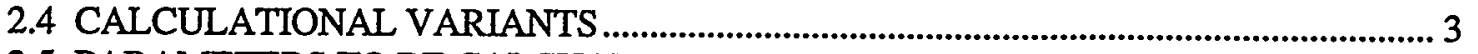

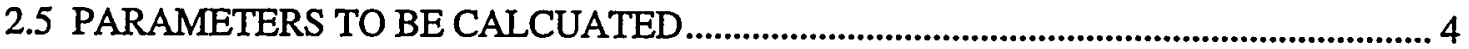

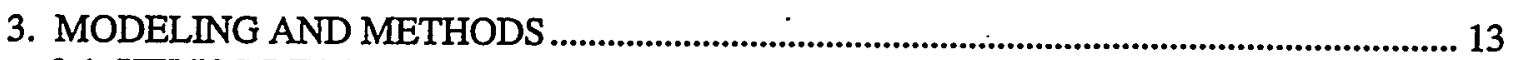

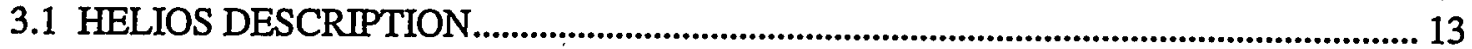

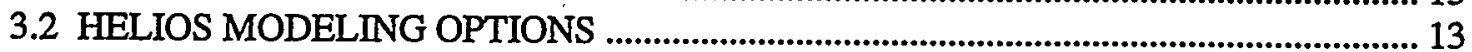

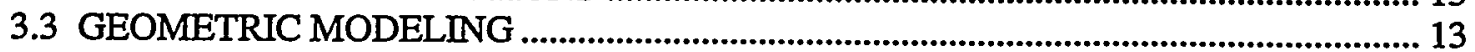

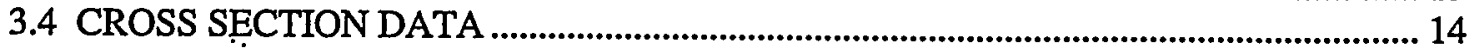

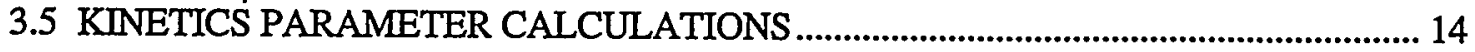

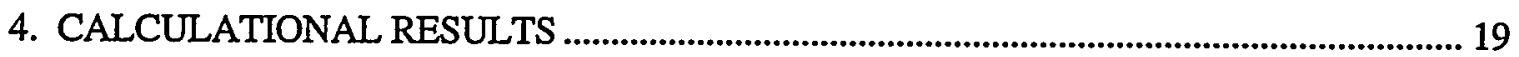

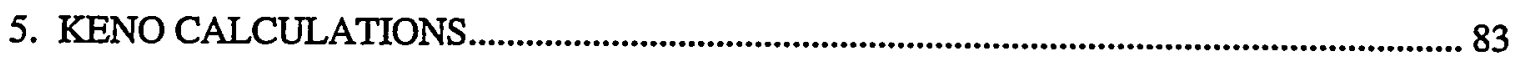

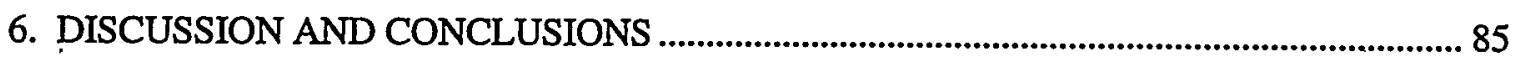

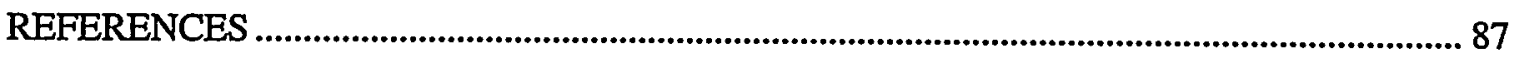


Page Intentionally Blank

I 


\section{LIST OF FIGURES}

Figure

Page

2.1 Diagram of $\mathrm{K} 331$ assembly design showing cell locations and types..................... 10

$2.2 \quad$ Geometry of MK2 multiassembly structure .................................................................. 11

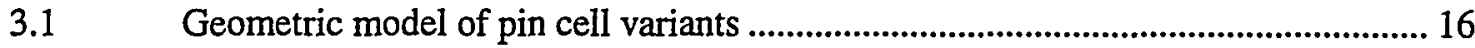

3.2 Geometric model of assembly variants (one-sixth assembly) ................................ 16

3.3 Geometric model of multiassembly structure..................................................... 17

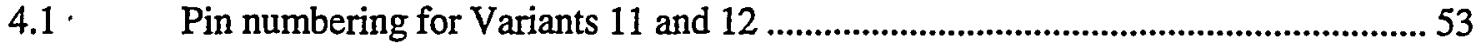

4.2 Relative fission rate distribution for Variant 11, State 1, at $0 \mathrm{MWd} / \mathrm{kg} \ldots \ldots \ldots \ldots \ldots \ldots . . . . .53$

4.3 Relative fission rate distribution for Variant 11, State 1, at $10 \mathrm{MWd} / \mathrm{kg} \ldots \ldots . . . . . . . . . .54$

4.4 Relative fission rate distribution for Variant 11, State 1, at $20 \mathrm{MWd} / \mathrm{kg}$................. 54

4.5 Relative fission rate distribution for Variant 11, State 1, at $30 \mathrm{MWd} / \mathrm{kg}$................ 55

4.6 Relative fission rate distribution for Variant 11, State 1, at $40 \mathrm{MWd} / \mathrm{kg}$................. 55

4.7 Relative fission rate distribution for Variant 11, State 1, at $50 \mathrm{MWd} / \mathrm{kg} \ldots \ldots \ldots \ldots . . . . .56$

4.8 Relative fission rate distribution for Variant 11, State 1, at $60 \mathrm{MWd} / \mathrm{kg}$.................56

4.9 Relative fission rate distribution for Variant 11, State 2, at $0 \mathrm{MWd} / \mathrm{kg} \ldots . . . . . . . . . . . . . .55$

4.10 Relative fission rate distribution for Variant 11, State 2, at $10 \mathrm{MWd} / \mathrm{kg}$................ 57

4.11 Relative fission rate distribution for Variant 11, State 2, at $20 \mathrm{MWd} / \mathrm{kg} \ldots \ldots \ldots \ldots . . . . . .58$

4.12 Relative fission rate distribution for Variant 11, State 2, at $30 \mathrm{MWd} / \mathrm{kg}$................. 58

4.13 Relative fission rate distribution for Variant 11, State 2, at $40 \mathrm{MWd} / \mathrm{kg}$................ 59

4.14 Relative fission rate distribution for Variant 11, State 2, at $50 \mathrm{MWd} / \mathrm{kg}$................. 59

4.15 Relative fission rate distribution for Variant 11, State 2, at $60 \mathrm{MWd} / \mathrm{kg}$................ 60

4.16 Relative fission rate distribution for Variant 12, State 1, at $0 \mathrm{MWd} / \mathrm{kg}$.................. 60

4.17 Relative fission rate distribution for Variant 12, State 1, at $10 \mathrm{MWd} / \mathrm{kg}$................. 61

4.18 Relative fission rate distribution for Variant 12, State 1, at $20 \mathrm{MWd} / \mathrm{kg}$................. 61

4.19 Relative fission rate distribution for Variant 12, State 1, at $30 \mathrm{MWd} / \mathrm{kg}$................. 62

4.20 Relative fission rate distribution for Variant 12, State 1, at $40 \mathrm{MWd} / \mathrm{kg}$.................. 62

4.21 Relative fission rate distribution for Variant 12, State 1, at $50 \mathrm{MWd} / \mathrm{kg}$..................63

4.22 Relative fission rate distribution for Variant 12, State 1, at $60 \mathrm{MWd} / \mathrm{kg}$.................. 63

4.23 Relative fission rate distribution for Variant 12, State 2, at $0 \mathrm{MWd} / \mathrm{kg} . . . . . . . . . . . . . . . . .64$

4.24 Relative fission rate distribution for Variant 12, State 2, at $10 \mathrm{MWd} / \mathrm{kg}$................ 64

4.25 Relative fission rate distribution for Variant 12, State 2, at $20 \mathrm{MWd} / \mathrm{kg}$................. 65

4.26 Relative fission rate distribution for Variant 12, State 2, at $30 \mathrm{MWd} / \mathrm{kg} \ldots \ldots \ldots \ldots \ldots . . . . . .65$

4.27 Relative fission rate distribution for Variant 12, State 2, at $40 \mathrm{MWd} / \mathrm{kg} . . . . . . . . . . . . . . .666$

4.28 Relative fission rate distribution for Variant 12, State 2, at $50 \mathrm{MWd} / \mathrm{kg}$................ 66

4.29 Relative fission rate distribution for Variant 12, State 2, at $60 \mathrm{MWd} / \mathrm{kg}$..................67

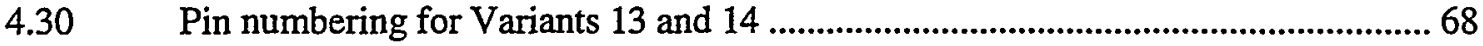

4.31 Relative fission rate distribution for Variant 13, State 1, at $0 \mathrm{MWd} / \mathrm{kg}$................... 68

4.32 Relative fission rate distribution for Variant 13, State 1, at $10 \mathrm{MWd} / \mathrm{kg}$..................69

4.33 Relative fission rate distribution for Variant 13, State 1, at $20 \mathrm{MWd} / \mathrm{kg}$................. 69

4.34 Relative fission rate distribution for Variant 13, State 1, at $30 \mathrm{MWd} / \mathrm{kg}$................. 70

4.35 Relative fission rate distribution for Variant 13, State 1, at $40 \mathrm{MWd} / \mathrm{kg}$................ 70

4.36 Relative fission rate distribution for Variant 13, State 1, at $50 \mathrm{MWd} / \mathrm{kg}$................ 71

4.37 Relative fission rate distribution for Variant 13, State 1, at $60 \mathrm{MWd} / \mathrm{kg}$.................. 71

4.38 Relative fission rate distribution for Variant 13, State 4, at $0 \mathrm{MWd} / \mathrm{kg}$................... 72

4.39 Relative fission rate distribution for Variant 13, State 4, at $10 \mathrm{MWd} / \mathrm{kg}$................. 72

4.40 Relative fission rate distribution for Variant 13, State 4, at $20 \mathrm{MWd} / \mathrm{kg}$................ 73

4.41 Relative fission rate distribution for Variant 13, State 4, at $30 \mathrm{MWd} / \mathrm{kg} \ldots \ldots \ldots \ldots . . . . . .73$

4.42 Relative fission rate distribution for Variant 13, State 4, at $40 \mathrm{MWd} / \mathrm{kg} . . . . . . . . . . . . . . .74$ 
4.43 Relative fission rate distribution for Variant 13, State 4, at $50 \mathrm{MWd} / \mathrm{kg} \ldots \ldots \ldots \ldots \ldots . . . . .74$

$4.44 \quad$ Relative fission rate distribution for Variant 13, State 4, at $60 \mathrm{MWd} / \mathrm{kg}$................ 75

$4.45 \quad$ Relative fission rate distribution for Variant 14, State 1, at $0 \mathrm{MWd} / \mathrm{kg} \ldots \ldots \ldots \ldots \ldots . . . . . . .75$

4.46 Relative fission rate distribution for Variant 14, State 1, at $10 \mathrm{MWd} / \mathrm{kg} \ldots \ldots \ldots \ldots \ldots . . . . .76$

4.47 Relative fission rate distribution for Variant 14, State 1, at $20 \mathrm{MWd} / \mathrm{kg}$................ 76

4.48 Relative fission rate distribution for Variant 14, State 1 , at $30 \mathrm{MWd} / \mathrm{kg}$................ 77

4.49 Relative fission rate distribution for Variant 14, State 1, at $40 \mathrm{MWd} / \mathrm{kg}$................. 77

4.50 Relative fission rate distribution for Variant 14, State 1, at $50 \mathrm{MWd} / \mathrm{kg}$................. 78

4.51 Relative fission rate distribution for Variant 14, State 1, at $60 \mathrm{MWd} / \mathrm{kg}$................. 78

4.52 Relative fission rate distribution for Variant 14, State 6, at $0 \mathrm{MWd} / \mathrm{kg} \ldots \ldots . . . . . . . . . . .79$

4.53 Relative fission rate distribution for Variant 14, State 6, at $10 \mathrm{MWd} / \mathrm{kg} \ldots \ldots \ldots \ldots . . . .79$

4.54 Relative fission rate distribution for Variant 14, State 6, at $20 \mathrm{MWd} / \mathrm{kg}$................ 80

4.55 Relative fission rate distribution for Variant 14, State 6 , at $30 \mathrm{MWd} / \mathrm{kg}$................ 80

4.56 Relative fission rate distribution for Variant 14, State 6, at $40 \mathrm{MWd} / \mathrm{kg}$................ 81

4.57 Relative fission rate distribution for Variant 14, State 6, at $50 \mathrm{MWd} / \mathrm{kg}$............... 81

4.58 Relative fission rate distribution for Variant 14, State 6, at $60 \mathrm{MWd} / \mathrm{kg}$................ 82

$6.1 \quad$ Variant 14 relative pin power along assembly axis.............................................. 86 


\section{LIST OF TABLES}

Table

Page

2.1

Material specifications

.5

2.2

Cell types. 7

2.3

State parameters 7

Specification of pin cell variants

.8

Specification of assembly variants

Multiassembly variant specifications.

Actinides and FPs in HELIOS library

Comparison of $k_{\text {inf }}$ values from the 34-, 89-, and 190-group libraries

Values of $k_{\text {eff }}$ and $k_{0}$ at zero burnup point for Variants $1-4,7-14$, States 1-6.

Values of $\mathrm{k}_{\mathrm{eff}}, \mathrm{k}_{0}$ and migration area vs burnup for Variants $1,2,10-12$,

State 1

Values of $\mathrm{k}_{\mathrm{eff}}, \mathrm{k}_{0}$, and migration area vs burnup for Variants $1,2,10-12$,

States 1-6.

Cell macroscopic cross sections, flux, and flux ratios vs burnup,

Variants $1-4$ and 7-10, State 1

4.5 Cell macroscopic cross sections, flux, and flux ratios vs burnup,

Variant 11, States 1 and 2 .

Cell macroscopic cross sections, flux, and flux ratios vs burnup,

Variant 12, States 1 and 2.

Isotopic concentration vs burnup for Variant 1 , State 1 .

Isotopic concentration vs burnup for Variant 2, State 1

Fuel-averaged microscopic absorption cross section for Variant 11,

States 1 and 2 .

Fuel-averaged microscopic absorption cross section for Variant 12,

States 1 and 2

4.21 Cell relative production reaction rate for uranium and plutonium isotopes vs burnup

4.22 Cell relative production reaction rates for Variant 11, States 1 and $2 \ldots \ldots \ldots \ldots \ldots . . . . . . . . .41$

Fuel-averaged microscopic production cross section for Variant 12,

States 1 and 2 .

4.26 Fuel-averaged microscopic absorption cross sections for uranium and plutonium isotopes vs burnup for Variants $1-4$ and $1-10$, State 1

4.27 Fuel-averaged microscopic production cross section for uranium and plutonium isotopes vs burnup, Variant $1-4$ and $7-10$, State 1 


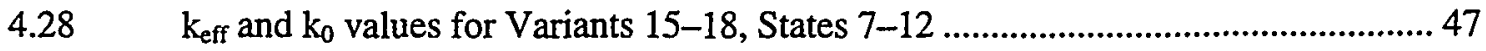

4.29 Values of critical buckling for Variants 15-18, States 11 and 12 ............................ 47

4.30 Fuel-averaged microscopic production cross sections $\left(v \sigma_{f}\right)$ for uranium and plutonium isotopes for Variants $15-18$................................................................. 48

4.31 Cell relative production rates for uranium and plutonium isotopes

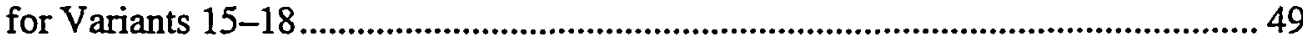

4.32 Fuel-averaged microscopic absorption cross sections $\left(\sigma_{\mathrm{a}}\right)$ for uranium

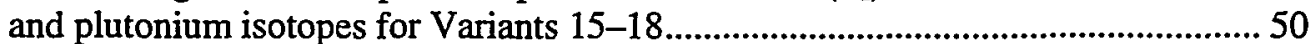

4.33 Cell relative absorption rates in uranium and plutonium isotopes for Variants $15-18$

4.34 Delayed neutron fraction $(\beta)$, effective delayed neutron fraction $\left(\beta_{\mathrm{eff}}\right)$, and prompt neutron lifetime for Variants $15-18$....................................................... 52

5.1 Comparison of HELIOS and KENO-VI $k_{\text {eff }}$ results for Variant 14 , State $1 \ldots \ldots . . . . .83$ 


\title{
CALCULATION OF THE RUSSIAN VVER-1000 BENCHMARKS WITH HELIOS AND KENO
}

\author{
J. C. Gehin \\ C. Dourougie \\ M. B. Emmett \\ R. A. Lillie
}

\begin{abstract}
Calculations of computational benchmark problems for the disposition of weapons-grade mixed-oxide fuel in VVER-1000 reactors have been performed with the HELIOS fuel assembly analysis code. The benchmarks cover pin cell, single fuel assembly, and multiassembly structures with several different fuel types, moderator densities, and boron content for operational and offnormal conditions. Fuel depletion is performed to a burnup of $60 \mathrm{MWd} / \mathrm{kg}$. The detailed results have been tabulated for multiplication factors, macroscopic and microscopic cross sections, reaction rates, fuel isotopics, and fission distributions. Additional results are given for a preliminary analysis of a multiassembly variant using the KENO Monte Carlo code with cross sections from HELIOS.
\end{abstract}




\section{Page Intentionally Blank}




\section{INTRODUCTION}

As part of the effort to eliminate surplus weapons plutonium, the options for burning the material in commercial power reactors will be analyzed. In Russia, a primary candidate for the disposition is the VVER-1000 reactor. To ensure that our existing calculational methods and data can accurately model VVER-1000 reactors with mixed-oxide (MOX) fuel of high-grade plutonium $\left(>90 \%{ }^{239} \mathrm{Pu}\right)$, benchmarking is required. Recently, many computational and experimental benchmarks have been analyzed ${ }^{1}$ in Russia. The computational benchmarks were formulated for detailed comparisons of calculational methods and data. This report documents the results of these benchmarks using the HELIOS ${ }^{2,3}$ fuel assembly analysis code.

The goal of this work is to perform calculations of the VVER-1000 computational benchmarks for direct comparison of the methods in use at Oak Ridge National Laboratory (ORNL) with those in use in Russia. The purpose of this benchmarking is to ensure that the U.S. and Russian methods agree early in the process so that fewer problems arise in the design and analysis process.

The benchmark specifications consist of a total of 20 calculational variants. The geometry of these variants includes single pin cells, single fuel assemblies, and a multiassembly structure for investigation of $\mathrm{UO}_{2}$ and $\mathrm{MOX}$ interface issues. Several fuel materials representing $\mathrm{UO}_{2}$, weapons-grade (WG) MOX, and reactor-grade (RG) MOX are considered. The conditions are typical of operation and off-normal states. Fuel depletion of $60 \mathrm{MWd} / \mathrm{kg}$ is performed for several of the variants. A detailed description of the benchmark specifications is given in Chap. 2 .

Calculations of the VVER benchmark variants were performed at ORNL using the HELIOS fuel assembly analysis code. The HELIOS code is very flexible and allows for precise modeling of benchmark problems. A discussion of the models and methods is presented in Chap. 3. The final calculational results are presented in Chap. 4.

Preliminary calculations of one of the multiassembly configurations were also performed with the KENO ${ }^{4}$ Monte Carlo code with cross sections from HELIOS. The results of these calculations are given in Chap. 5. A final discussion of the work is presented in Chap. 6. 
Page Intentionally Blank 


\section{BENCHMARK SPECIFICATIONS}

Two sets of benchmark variants were formulated by the Russian participants in the joint U.S./Russian Federation plutonium disposition program. The first set ${ }^{1}$ covered different aspects of $\mathrm{UO}_{2}$ and MOX in VVER-1000 reactors in normal operating conditions. The second set ${ }^{5}$ was formulated to cover off-normal conditions and provide for a comparison of kinetics parameters. Specifications for these benchmark problems were formulated by the Russian participants in the joint U.S./Russian Federation plutonium disposition program. The following specifications are a combination of these two sets of benchmark variants.

\subsection{MATERIAL SPECIFICATIONS}

Material specifications for the different fuel materials, cladding, absorbers, and moderators are given in Table 2.1. The fuel materials include fresh $\mathrm{UO}_{2}$ (FU1), fresh WG MOX(FU2), and RG MOX (FU10); spent $\mathrm{UO}_{2}$ (FU3 and FU4) and WG MOX (FU11); fresh MOX with individual plutonium isotopes (FU7-FU9); and WG MOX with different plutonium contents for graded fuel designs (FU12-FU14). The cladding material is zirconium (CL1), and the absorber material is $\mathrm{B}_{4} \mathrm{C}(\mathrm{ABS})$. Six different types of moderator materials differing in density and boron concentration are given (MOD1-MOD6).

\subsection{GEOMETRY SPECIFICATIONS}

The geometry consists of different basic cell types (fuel, absorber, central tube, and guide tube), a standard VVER-1000 hexagonal assembly with 331 cell locations, and a multiassembly structure formed by two different assembly types. The cell types are presented in Table 2.2. Each cell type consists of concentric cylinders of materials in a hexagonal region. The assembly configuration, referred to as the K331 assembly, is shown in Fig. 2.1. The flat-to-flat dimension of the "container" zone is $23.6 \mathrm{~cm}$, which represents the interassembly spacing. The multiassembly structure (MK2) is formed by an infinite array of two assembly types (K1 and K2), as shown in Fig. 2.2. The resulting ratio of $K 2$ to $K 1$ assemblies is 3:1. Note that the container zone shown in Fig. 2.1 is intended only to represent the assembly pitch; there is no physical container surrounding the VVER-1000 assemblies.

\subsection{STATE SPECIFICATIONS}

The specification of the temperatures, moderator density and boron content, absorber presence, and fission product (FP) poisoning is indicated by the different states. For these benchmark problems, twelve states are used, as presented in Table 2.3. Fuel burnup calculations are performed with State 1 only, and the remaining states are used for branch calculations (for every $10-\mathrm{MWd} / \mathrm{kg}$ step). The power density for the burnup calculations is $108 \mathrm{MW} / \mathrm{m}^{3}$ taken over the entire volume (fuel, cladding, moderator, etc.).

\subsection{CALCULATIONAL VARIANTS}

The variants to be calculated consist of different combinations of the material, geometric, and state specifications given previously. The specific variants are presented in Tables 2:4 and 2.5. The first set of variants (V1-V14) represent standard operating conditions, while the second set (V15-V20) represent off-normal and accident conditions. 


\subsection{PARAMETERS TO BE CALCULATED}

quantities:

The parameters to be calculated are variant-dependent and consist of the following

(a) The $\mathrm{k}_{\text {eff }}$ or critical buckling, $\mathrm{k}_{0}$ (ratio of neutron production to neutron absorption).

(b) Migration area, $\mathrm{M}^{2}$.

(c) Concentrations of ${ }^{235} \mathrm{U},{ }^{236} \mathrm{U},{ }^{238} \mathrm{U},{ }^{238} \mathrm{Pu},{ }^{239} \mathrm{Pu},{ }^{240} \mathrm{Pu},{ }^{241} \mathrm{Pu},{ }^{242} \mathrm{Pu},{ }^{135} \mathrm{Xe},{ }^{149} \mathrm{Sm}$, and total concentrations of FPs in fuel (for assembly geometry type in cells numbered 1, 29, and 72 in Fig. 2.1).

(d) Concentrations of ${ }^{241} \mathrm{Am},{ }^{242 \mathrm{~m}} \mathrm{Am},{ }^{243} \mathrm{Cm}$, and ${ }^{244} \mathrm{Cm}$ in fuel.

(e) Pin-by-pin power (fission) distribution.

(f) Neutron flux in the cell and the ratio of the neutron flux in the fuel, cladding, and moderator regions to that of the cell.

(g) One-group macroscopic cross sections $\left(\Sigma_{\mathrm{a}}, \Sigma_{\mathrm{f}}, \mathrm{v} \Sigma_{\mathrm{f}}\right)$ averaged over the cell (for the assembly geometry types in cells numbered 1,29 , and 72 in Fig. 2.1).

(h) Fuel-averaged, one-group microscopic cross sections $\left(\sigma_{\mathrm{a}}\right.$ and $\left.v \sigma_{f}\right)$ of ${ }^{235} \mathrm{U},{ }^{238} \mathrm{U},{ }^{238} \mathrm{Pu}$, ${ }^{239} \mathrm{Pu},{ }^{240} \mathrm{Pu},{ }^{241} \mathrm{Pu},{ }^{242} \mathrm{Pu},{ }^{135} \mathrm{Xe},{ }^{149} \mathrm{Sm}$, and total FPs and relative reactions for these nuclides in the cell (for the assembly geometry type in cells numbered 1,29, and 72).

(i) Real and effective delayed neutron fractions ( $\beta$ and $\beta_{\text {eff }}$ ).

(j) Prompt neutron lifetime $(\Lambda)$.

The particular parameters to be computed for each variant are indicated in Tables 2.4-2.6. Note that for the variants that require burnup, items (a-d) are to be provided at every $2 \mathrm{MWd} / \mathrm{kg}$ for State 1 and every $10 \mathrm{MWd} / \mathrm{kg}$ for the remaining states. Items $(\mathrm{e}-\mathrm{h})$ are to be provided at every $10 \mathrm{MWd} / \mathrm{kg}$. 
Table 2.1. Material specifications

\begin{tabular}{|c|c|c|c|c|c|c|c|c|c|}
\hline Material & Description & Isotope & $\begin{array}{c}\text { Content } \\
\text { (atoms } / \mathrm{b} \cdot \mathrm{cm})\end{array}$ & Isotope & $\begin{array}{c}\text { Content } \\
\text { (atoms } / \mathrm{b} \cdot \mathrm{cm})\end{array}$ & Isotope & $\begin{array}{c}\text { Content } \\
\text { (atoms } / \mathrm{b} \cdot \mathrm{cm})\end{array}$ & Isotope & $\begin{array}{c}\text { Content } \\
(\text { atoms } / \mathrm{b} \cdot \mathrm{cm})\end{array}$ \\
\hline FUl & Fresh uranium fuel & ${ }^{235} \mathrm{U}$ & $8.7370 \times 10^{-4}$ & ${ }^{238} \mathrm{U}$ & $1.8744 \times 10^{-2}$ & ${ }^{16} \mathrm{O}$ & $3.9235 \times 10^{-2}$ & & \\
\hline $\mathrm{FU} 2$ & Fresh MOX fuel & ${ }^{238} \mathrm{U}$ & $\begin{array}{l}3.8393 \times 10^{-5} \\
1.8917 \times 10^{-2}\end{array}$ & ${ }^{239} \mathrm{Pu}$ & $\begin{array}{l}6.5875 \times 10^{-4} \\
4.2323 \times 10^{-5}\end{array}$ & ${ }^{241} \mathrm{Pu}$ & $7.0246 \times 10^{-6}$ & ${ }^{16} \mathrm{O}$ & $4.1707 \times 10^{-2}$ \\
\hline FU3 & $\begin{array}{l}\text { Spent uranium fuel without fission } \\
\text { products } \\
\quad \text {. }\end{array}$ & 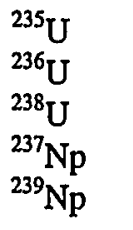 & $\begin{array}{l}3.7843 \times 10^{-4} \\
8.6365 \times 10^{-5} \\
1.8327 \times 10^{-2} \\
2.4823 \times 10^{-5} \\
1.8332 \times 10^{-6}\end{array}$ & $\begin{array}{l}{ }^{238} \mathrm{Pu} \\
{ }^{239} \mathrm{Pu} \\
{ }^{241} \mathrm{Pu} \\
\mathrm{Pu}\end{array}$ & $\begin{array}{l}6.7254 \times 10^{-6} \\
1.3111 \times 10^{-4} \\
3.6233 \times 10^{-5} \\
2.1701 \times 10^{-5}\end{array}$ & $\begin{array}{l}{ }^{242} \mathrm{Pu} \\
{ }^{241} \mathrm{Am} \\
{ }^{242 \mathrm{~m}} \mathrm{Am} \\
{ }^{243} \mathrm{Am}\end{array}$ & $\begin{array}{l}4.7576 \times 10^{-6} \\
4.9491 \times 10^{-7} \\
7.9194 \times 10^{-9} \\
6.6925 \times 10^{-7}\end{array}$ & $\begin{array}{l}{ }^{242} \mathrm{Cm} \\
{ }^{243} \mathrm{Cm} \\
{ }^{244} \mathrm{Cm} \\
{ }^{16} \mathrm{O}\end{array}$ & $\begin{array}{l}1.2582 \times 10^{-7} \\
2.0629 \times 10^{-9} \\
1.2387 \times 10^{-7} \\
3.9235 \times 10^{-2}\end{array}$ \\
\hline FU4 & Spent uranium fuel with fission products & $\begin{array}{l}{ }^{235} \mathrm{U} \\
{ }^{236} \mathrm{U} \\
{ }^{238} \mathrm{U} \\
{ }^{237} \mathrm{~Np} \\
{ }^{238} \mathrm{Pu} \\
{ }^{239} \mathrm{~Np} \\
{ }^{239} \mathrm{Pu} \\
{ }^{240} \mathrm{Pu}\end{array}$ & $\begin{array}{l}3.7843 \times 10^{-4} \\
8.6365 \times 10^{-5} \\
1.8327 \times 10^{-2} \\
2.4823 \times 10^{-5} \\
6.7254 \times 10^{-6} \\
1.8332 \times 10^{-6} \\
1.3111 \times 10^{-4} \\
3.6233 \times 10^{-5}\end{array}$ & $\begin{array}{l}{ }^{241} \mathrm{Pu} \\
{ }^{242} \mathrm{Pu} \\
{ }^{241} \mathrm{Am} \\
{ }^{242 \mathrm{~m}} \mathrm{Am} \\
{ }^{243} \mathrm{Am} \\
{ }^{242} \mathrm{Cm} \\
{ }^{243} \mathrm{Cm} \\
{ }^{244} \mathrm{Cm}\end{array}$ & $\begin{array}{l}2.1701 \times 10^{-5} \\
4.7576 \times 10^{-6} \\
4.9491 \times 10^{-7} \\
7.9194 \times 10^{-9} \\
6.6925 \times 10^{-7} \\
1.2582 \times 10^{-7} \\
2.0629 \times 10^{-9} \\
1.2387 \times 10^{-7}\end{array}$ & $\begin{array}{l}{ }^{95} \mathrm{Mo} \\
{ }^{99} \mathrm{Tc} \\
{ }^{101} \mathrm{Ru} \\
{ }^{103} \mathrm{Rh} \\
{ }^{109} \mathrm{Ag} \\
{ }^{131} \mathrm{Xe} \\
{ }^{133} \mathrm{Cs} \\
{ }^{143} \mathrm{Nd}\end{array}$ & $\begin{array}{l}3.3720 \times 10^{-5} \\
3.3320 \times 10^{-5} \\
3.1134 \times 10^{-5} \\
1.8890 \times 10^{-5} \\
2.2037 \times 10^{-6} \\
1.4255 \times 10^{-5} \\
3.5974 \times 10^{-5} \\
2.6692 \times 10^{-5}\end{array}$ & $\begin{array}{l}{ }^{145} \mathrm{Nd} \\
{ }^{147} \mathrm{Pm} \\
{ }^{151} \mathrm{Sm} \\
{ }^{152} \mathrm{Sm} \\
{ }^{153} \mathrm{Eu} \\
{ }^{155} \mathrm{Eu} \\
{ }^{154} \mathrm{Eu} \\
{ }^{16} \mathrm{O}\end{array}$ & $\begin{array}{l}1.9975 \times 10^{-5} \\
6.1574 \times 10^{-6} \\
3.0757 \times 10^{-7} \\
2.6842 \times 10^{-6} \\
2.4801 \times 10^{-6} \\
9.6857 \times 10^{-8} \\
5.1189 \times 10^{-7} \\
3.9235 \times 10^{-2}\end{array}$ \\
\hline FU7 & Fresh MOX fuel with ${ }^{239} \mathrm{Pu}$ only & ${ }^{235} \mathrm{U}$ & $3.8393 \times 10^{-5}$ & ${ }^{238} \mathrm{U}$ & $1.8917 \times 10^{-2}$ & ${ }^{239} \mathrm{Pu}$ & $6.5875 \times 10^{-4}$ & ${ }^{16} \mathrm{O}$ & $4.1707 \times 10^{-2}$ \\
\hline FU8 & Fresh MOX fuel with ${ }^{240} \mathrm{Pu}$ only & ${ }^{235} \mathrm{U}$ & $3.8393 \times 10^{-5}$ & ${ }^{238} \mathrm{U}$ & $1.8917 \times 10^{-2}$ & ${ }^{240} \mathrm{Pu}$ & $4.2323 \times 10^{-5}$ & ${ }^{16} \mathrm{O}$ & $4.1707 \times 10^{-2}$ \\
\hline FU9 & Fresh MOX fuel with ${ }^{24 !} \mathrm{Pu}$ only & ${ }^{235} \mathrm{U}$ & $3.8393 \times 10^{-5}$ & ${ }^{238} \mathrm{U}$ & $1.8917 \times 10^{-2}$ & ${ }^{24 I} \mathrm{Pu}$ & $6.6577 \times 10^{-4}$ & ${ }^{16} \mathrm{O}$ & $4.1707 \times 10^{-2}$ \\
\hline FU10 & Fresh MOX fuel (RG) & $\begin{array}{l}{ }^{235} \mathrm{U} \\
{ }^{238} \mathrm{U} \\
{ }^{238} \mathrm{Pu}\end{array}$ & $\begin{array}{l}5.0000 \times 10^{-5} \\
2.2100 \times 10^{-2} \\
3.0000 \times 10^{-5}\end{array}$ & $\begin{array}{l}{ }^{239} \mathrm{Pu} \\
{ }^{240} \mathrm{Pu}\end{array}$ & $\begin{array}{l}1.1600 \times 10^{-3} \\
4.9000 \times 10^{-4}\end{array}$ & ${ }^{241} \mathrm{Pu}$ & $\begin{array}{l}1.9000 \times 10^{-4} \\
1.0500 \times 10^{-4}\end{array}$ & ${ }^{241} \mathrm{Am}$ & $\begin{array}{l}2.5000 \times 10^{-5} \\
4.6300 \times 10^{-2}\end{array}$ \\
\hline
\end{tabular}


Table 2.1. Material specifications (continued)

\begin{tabular}{|c|c|c|c|c|c|c|c|c|c|}
\hline Material & Description & Isotope & $\begin{array}{c}\text { Content } \\
\text { (atoms } / \mathrm{b} \cdot \mathrm{cm} \text { ) }\end{array}$ & Isotope & $\begin{array}{c}\text { Content } \\
(\text { atoms } / \mathrm{b} \cdot \mathrm{cm})\end{array}$ & Isotope & $\begin{array}{c}\text { Content } \\
\text { (atoms } / \mathrm{b} \cdot \mathrm{cm} \text { ) }\end{array}$ & Isotope & $\begin{array}{c}\text { Content } \\
(\text { atoms } / \mathrm{b} \cdot \mathrm{cm})\end{array}$ \\
\hline FU11 & Spent plutonium fuel with fission products & $\begin{array}{l}{ }^{235} \mathrm{U} \\
{ }^{236} \mathrm{U} \\
{ }^{238} \mathrm{U} \\
{ }^{237} \mathrm{~Np} \\
{ }^{239} \mathrm{~Np} \\
{ }^{238} \mathrm{Pu} \\
{ }^{239} \mathrm{Pu} \\
{ }^{240} \mathrm{Pu}\end{array}$ & $\begin{array}{l}1.9990 \times 10^{-5} \\
3.5520 \times 10^{-6} \\
1.8480 \times 10^{-2} \\
2.0710 \times 10^{-6} \\
2.0200 \times 10^{-6} \\
1.1180 \times 10^{-6} \\
3.2190 \times 10^{-4} \\
1.4640 \times 10^{-4}\end{array}$ & $\begin{array}{l}{ }^{241} \mathrm{Pu} \\
{ }^{242} \mathrm{Pu} \\
{ }^{241} \mathrm{Am} \\
{ }^{242 \mathrm{~m}} \mathrm{Am} \\
{ }^{243} \mathrm{Am} \\
{ }^{242} \mathrm{Cm} \\
{ }^{243} \mathrm{Cm} \\
{ }^{244} \mathrm{Cm}\end{array}$ & $\begin{array}{l}7.6080 \times 10^{-5} \\
1.7300 \times 10^{-5} \\
2.6120 \times 10^{-6} \\
4.4530 \times 10^{-8} \\
3.2580 \times 10^{-6} \\
6.2890 \times 10^{-7} \\
1.4150 \times 10^{-8} \\
8.0640 \times 10^{-7}\end{array}$ & $\begin{array}{l}{ }^{95} \mathrm{Mo} \\
{ }^{99} \mathrm{Tc} \\
{ }^{101} \mathrm{Ru} \\
{ }^{103} \mathrm{Rh} \\
{ }^{109} \mathrm{Ag} \\
{ }^{131} \mathrm{Xe} \\
{ }^{133} \mathrm{Cs} \\
{ }^{143} \mathrm{Nd}\end{array}$ & $\begin{array}{l}1.3470 \times 10^{-5} \\
1.6610 \times 10^{-5} \\
1.7120 \times 10^{-5} \\
1.5060 \times 10^{-5} \\
3.4130 \times 10^{-6} \\
8.1400 \times 10^{-6} \\
1.8250 \times 10^{-5} \\
1.1490 \times 10^{-5}\end{array}$ & $\begin{array}{l}{ }^{145} \mathrm{Nd} \\
{ }^{147} \mathrm{Pm} \\
{ }^{151} \mathrm{Sm} \\
{ }^{152} \mathrm{Sm} \\
{ }^{153} \mathrm{Eu} \\
{ }^{154} \mathrm{Eu} \\
{ }^{155} \mathrm{Eu} \\
{ }^{16} \mathrm{O}\end{array}$ & $\begin{array}{l}8.4160 \times 10^{-6} \\
2.8310 \times 10^{-6} \\
3.4630 \times 10^{-7} \\
2.0600 \times 10^{-6} \\
2.0570 \times 10^{-6} \\
5.1640 \times 10^{-7} \\
8.8120 \times 10^{-8} \\
4.1707 \times 10^{-2}\end{array}$ \\
\hline FU12 & MOX fuel of type 1 in zoned fuel assembly & ${ }^{233} \mathrm{U}$ & $\begin{array}{l}3.7918 \times 10^{-5} \\
1.8683 \times 10^{-2}\end{array}$ & ${ }^{239} \mathrm{Pu}$ & $\begin{array}{l}8.7697 \times 10^{-4} \\
5.6343 \times 10^{-5}\end{array}$ & ${ }^{241} \mathrm{Pu}$ & $9.3516 \times 10^{-6}$ & ${ }^{16} \mathrm{O}$ & $4.1707 \times 10^{-2}$ \\
\hline FU13 & MOX fuel of type 2 in zoned fuel assembly & ${ }^{235} \mathrm{U} U$ & $\begin{array}{l}3.8786 \times 10^{-5} \\
1.9111 \times 10^{-2}\end{array}$ & ${ }^{239} \mathrm{Pu}$ & $\begin{array}{l}4.7835 \times 10^{-4} \\
3.0733 \times 10^{-5}\end{array}$ & ${ }^{241} \mathrm{Pu}$ & $5.1009 \times 10^{-6}$ & ${ }^{16} \mathrm{O}$ & $4.1707 \times 10^{-2}$ \\
\hline FU14 & MOX fuel of type 3 in zoned fuel assembly & ${ }^{2335} U$ & $\begin{array}{l}3.8959 \times 10^{-5} \\
1.9196 \times 10^{-2}\end{array}$ & ${ }^{230} \mathrm{Pu}$ & $\begin{array}{l}3.9862 \times 10^{-4} \\
2.5610 \times 10^{-5}\end{array}$ & ${ }^{241} \mathrm{Pu}$ & $4.2507 \times 10^{-6}$ & ${ }^{16} \mathrm{O}$ & $4.1707 \times 10^{-2}$ \\
\hline CL1 & Cladding & $\mathrm{Zr}$ & $4.2300 \times 10^{-2}$ & & & & & & \\
\hline MOD1 & $\begin{array}{l}\text { Hot moderator with } 0.6 \mathrm{~g} / \mathrm{kg} \text { of natural } \\
\text { boron }\end{array}$ & $\mathrm{H}$ & $4.7830 \times 10^{-2}$ & ${ }^{16} \mathrm{O}$ & $2.3910 \times 10^{-2}$ & ${ }^{10} \mathrm{~B}$ & $4.7344 \times 10^{-6}$ & ${ }^{11} \mathrm{~B}$ & $1.9177 \times 10^{-5}$ \\
\hline MOD2 & Hot moderator without boron & $\mathrm{H}$ & $4.7830 \times 10^{-2}$ & ${ }^{16} \mathrm{O}$ & $2.3910 \times 10^{-2}$ & & & & \\
\hline MOD3 & $\begin{array}{l}\text { Cold moderator with } 0.6 \mathrm{~g} / \mathrm{kg} \text { of natural } \\
\text { boron }\end{array}$ & $\mathrm{H}$ & $6.6940 \times 10^{-2}$ & ${ }^{16} \mathrm{O}$ & $3.3470 \times 10^{-2}$ & ${ }^{10} \mathrm{~B}$ & $6.6262 \times 10^{-6}$ & ${ }^{11} \mathrm{~B}$ & $2.6839 \times 10^{-5}$ \\
\hline MOD4 & Hot moderator of low density & $\mathrm{H}$ & $1.3390 \times 10^{-2}$ & ${ }^{16} \mathrm{O}$ & $6.6900 \times 10^{-3}$ & & & & \\
\hline MOD5 & Cold moderator without natural boron & $\mathrm{H}$ & $6.6940 \times 10^{-2}$ & ${ }^{16} \mathrm{O}$ & $3.3470 \times 10^{-2}$ & & & & \\
\hline MOD6 & Hot moderator with $1.2 \mathrm{~g} / \mathrm{kg}$ of boron & $\mathrm{H}$ & $4.7830 \times 10^{-2}$ & ${ }^{16} \mathrm{O}$ & $2.3910 \times 10^{-2}$ & ${ }^{10} \mathrm{~B}$ & $9.4688 \times 10^{-6}$ & ${ }^{11} \mathrm{~B}$ & $3.8354 \times 10^{-5}$ \\
\hline
\end{tabular}


Table 2.2. Cell types

\begin{tabular}{clclc}
\hline \multirow{2}{*}{ Cell type } & \multirow{2}{*}{ Description } & \multicolumn{3}{c}{ Cell cylindrical zone specifications $^{a}$} \\
\cline { 3 - 5 } & Fumber & \multicolumn{1}{c}{ Description $^{b}$} & $\begin{array}{c}\text { Outer radius } \\
\text { (cm) }\end{array}$ \\
\hline G1 & Fuel cell & 1 & Fuel (FUn) & 0.386 \\
& & 2 & Clad (CL1) & 0.4582 \\
G2 & Central tube cell & 1 & Coolant (MODn) & 0.45 \\
& & 2 & Structure (CL1) & 0.51277 \\
G3 & \multirow{2}{*}{ Absorber cell } & 1 & Coolant (MODn) & 0.35 \\
& & 2 & Clad (CL1) & 0.41 \\
& & 3 & Absorber (ABS) & 0.545 \\
& & 4 & Clad (CL1) & 0.6323 \\
\hline G4 & Guide tube cell & 1 & Coolant (MODn) & 0.545 \\
& & 2 & Structure (CL1) & 0.6323 \\
\hline
\end{tabular}

${ }^{a}$ All cell types are surrounded by a moderator/coolant region with a hexagonal shape and a flat-to-flat dimension of $1.275 \mathrm{~cm}$.

${ }^{b}$ Values in parentheses refer to information in Table 2.1.

Table 2.3. State parameters

\begin{tabular}{lcccccc}
\hline State & $\begin{array}{c}\text { Fuel } \\
\text { temperature } \\
(\mathrm{K})\end{array}$ & $\begin{array}{c}\text { Nonfuel } \\
\text { temperature } \\
(\mathrm{K})\end{array}$ & $\begin{array}{c}\text { Moderator } \\
\text { material }\end{array}$ & $\begin{array}{c}\text { Cell type } \\
3^{a}\end{array}$ & $\begin{array}{c}\mathbf{1 3 5}_{\text {Xe and }}{ }^{149} \mathrm{Sm} \\
\text { FPs }^{b}\end{array}$ & $\begin{array}{c}\text { Buckling }^{{ }^{\mathbf{2}}} \\
\left(\mathrm{cm}^{-2}\right)\end{array}$ \\
\hline S1 & 1027 & 579 & MOD1 & G4 & Present & 0.003 \\
S2 & 1027 & 579 & MOD1 & G3 & Present & 0.003 \\
S3 & 1027 & 579 & MOD2 & G4 & None & 0.003 \\
S4 & 1027 & 579 & MOD1 & G4 & Present & 0.003 \\
S5 & 579 & 579 & MOD1 & G4 & None & 0.003 \\
S6 & 300 & 300 & MOD3 & G4 & None & 0.003 \\
S7 & 1027 & 579 & MOD2 & G4 & None & 0.0 \\
S8 & 2000 & 579 & MOD2 & G4 & None & 0.0 \\
S9 & 1027 & 579 & MOD4 & G4 & None & 0.0 \\
S10 & 1027 & 579 & MOD6 & G4 & None & 0.0 \\
S11 & 1027 & 579 & MOD2 & G4 & None & Critical \\
S12 & 300 & 300 & MOD5 & G4 & None & Critical \\
\hline
\end{tabular}

${ }^{a}$ See Fig. 2.1.

${ }^{b 135} \mathrm{Xe}$ and ${ }^{149} \mathrm{Sm}$ number densities are $9.4581 \times 10^{-9}$ and $7.3667 \times 10^{-8}$ atoms/(b.cm), respectively, at zero . burnup and equilibrium values at other burnups. 
Table 2.4. Specification of pin cell variants

\begin{tabular}{|c|c|c|c|c|c|}
\hline Variant & Description $^{a}$ & $\begin{array}{c}\text { Fuel } \\
\text { material }\end{array}$ & States $^{c}$ & $\begin{array}{l}\text { Maximum } \\
\text { burnup } \\
\text { (MWd } / \mathrm{kg} \text { ) }\end{array}$ & $\begin{array}{l}\text { Parameters } \\
\text { to compute }^{d}\end{array}$ \\
\hline $\mathrm{V} 1$ & $\mathrm{UO}_{2}$ pin cell & FU1 & S1, S3-S6 & 60 & (a)-(h) \\
\hline V2 & MOX pin cell & FU2 & S1, S3-S6 & 60 & (a)-(h) \\
\hline V3 & Spent $\mathrm{UO}_{2}$ pin cell, no FPs & FU3 & S1, S3-S6 & 0 & (a)-(h) \\
\hline V4 & Spent $\mathrm{UO}_{2}$ pin cell, with FPs & FU4 & S1, S3-S6 & 0 & (a)-(h) \\
\hline V7 & MOX pin cell with ${ }^{239} \mathrm{Pu}$ only & FU7 & S1, s3-S6 & 0 & (a)-(h) \\
\hline V8 & MOX pin cell with ${ }^{240} \mathrm{Pu}$ only & FU8 & S1, S3-S6 & 0 & (a)-(h) \\
\hline v9 & MOX pin cell with ${ }^{241} \mathrm{Pu}$ only & FU9 & S1, S3-S6 & 0 & (a)-(h) \\
\hline v10 & Reactor MOX pin cell & FU10 & S1, S3-S6 & 60 & (a)-(h) \\
\hline V15 & $\begin{array}{l}\mathrm{UO}_{2} \text { pin cell, accident conditions, } \\
\text { kinetics parameters }\end{array}$ & FU1 & S7-S12 & 0 & $(\mathrm{a}),(\mathrm{h})-(\mathrm{j})$ \\
\hline V16 & $\begin{array}{l}\text { Spent } \mathrm{UO}_{2} \text { pin cell, accident } \\
\text { conditions, kinetics parameters }\end{array}$ & FU4 & S7-S12 & 0 & (a), (h)-(j) \\
\hline V17 & $\begin{array}{l}\text { MOX pin cell, accident conditions, } \\
\text { kinetics parameters }\end{array}$ & FU2 & S7-S12 & 0 & (a), (h)-(j) \\
\hline V18 & $\begin{array}{l}\text { Spent MOX pin cell, accident } \\
\text { conditions, kinetics parameters }\end{array}$ & FU11 & S7-S12 & 0 & (a), (h)-(j) \\
\hline
\end{tabular}

${ }^{\circ}$ All cases have G1 cell geometry given in Table 2.2.

${ }^{b}$ Refer to Table 2.1 for material definitions.

Refer to Table 2.3 for state definitions.

${ }^{d}$ Parameter definitions given in Sect. 2.5 .

Table 2.5. Specification of assembly variants

\begin{tabular}{cccccccc}
\hline Variant & Description & $\begin{array}{c}\text { Assembly } \\
\text { cell }^{a}\end{array}$ & $\begin{array}{c}\text { Cell } \\
\text { type }^{b}\end{array}$ & $\begin{array}{c}\text { Fuel } \\
\text { material }^{c}\end{array}$ & States $^{d}$ & $\begin{array}{c}\text { Maximum } \\
\text { burnup } \\
\text { (MWd/kg) }\end{array}$ & $\begin{array}{c}\text { Parameters to } \\
\text { compute }^{e}\end{array}$ \\
\hline V11 & UO $_{2}$ assembly & $1,4,5$ & $\mathrm{G} 1$ & FU1 & S1-S6 & 60 & $(\mathrm{a})-(\mathrm{h})$ \\
& & 2 & $\mathrm{G} 2$ & & & & \\
& & 3 & $\mathrm{G} 3 / \mathrm{G} 4^{f}$ & & & & \\
V12 & MOX assembly & $1,4,5$ & $\mathrm{G} 1$ & FU1 & S1-S6 & 60 & (a)-(h) \\
& & 2 & $\mathrm{G} 2$ & & & & \\
\hline
\end{tabular}

${ }^{a}$ Refer to Fig. 2.1 for assembly cell locations.

${ }^{b}$ Refer to Table 2.2 for cell types.

Refer to Table 2.1 for material definitions.

${ }^{d}$ Refer to Table 2.3 for state definitions.

'Parameter definitions given in Sect. 2.5.

${ }^{f}$ Cell type G3 is used only for State 2; G4 is used for all other states. 
Table 2.6. Multiassembly variant specifications

\begin{tabular}{|c|c|c|c|c|c|c|c|}
\hline Variant & Description & Assembly $^{a}$ & $\begin{array}{l}\text { Assembly } \\
\text { cell }^{b}\end{array}$ & $\begin{array}{c}\text { Fuel } \\
\text { material }^{c}\end{array}$ & State $^{d}$ & $\begin{array}{l}\text { Maximum } \\
\text { burnup } \\
\text { (MWd/kg) }\end{array}$ & $\begin{array}{l}\text { Parameters to } \\
\text { compute }^{e}\end{array}$ \\
\hline V13 & $\begin{array}{l}\text { Multiassembly with uniform } \\
\text { MOX assembly }\end{array}$ & $\begin{array}{l}\mathrm{K} 1 \\
\mathrm{~K} 2\end{array}$ & $\begin{array}{l}1-5 \\
1-5\end{array}$ & $\begin{array}{l}\text { FU1 } \\
\text { FU2 }\end{array}$ & $\mathrm{S} 1, \mathrm{~S} 4$ & 60 & (a), (b), (e) \\
\hline V14 & $\begin{array}{l}\text { Multiassembly with graded } \\
\text { MOX assembly }\end{array}$ & $\begin{array}{l}\mathrm{K} 1 \\
\mathrm{~K} 2\end{array}$ & $\begin{array}{l}1-3 \\
4 \\
5 \\
1-5\end{array}$ & $\begin{array}{l}\text { FU12 } \\
\text { FU13 } \\
\text { FU14 } \\
\text { FU1 }\end{array}$ & S1, S6 & 60 & (a), (b), (e) \\
\hline V19 & $\begin{array}{l}\text { Multiassembly, accident } \\
\text { conditions, kinetics } \\
\text { parameters }\end{array}$ & $\begin{array}{l}\mathrm{K} 1 \\
\mathrm{~K} 2\end{array}$ & $\begin{array}{l}1-5 \\
1-5\end{array}$ & $\begin{array}{l}\text { FU2 } \\
\text { FU1 }\end{array}$ & S7-S12 & 0 & $\begin{array}{l}\text { (a), (b), (i), } \\
\text { (j) }\end{array}$ \\
\hline V20 & $\begin{array}{l}\text { Multiassembly with spent } \\
\mathrm{UO}_{2} \text { assemblies, accident } \\
\text { conditions, kinetics } \\
\text { parameters }\end{array}$ & $\begin{array}{l}\mathrm{K} 1 \\
\mathrm{~K} 2\end{array}$ & $\begin{array}{l}1-5 \\
1-5\end{array}$ & $\begin{array}{l}\text { FU2 } \\
\text { FU4 }\end{array}$ & S7-S12 & 0 & $\begin{array}{l}\text { (a), (b), (i), } \\
\text { (j) }\end{array}$ \\
\hline
\end{tabular}

${ }^{a}$ Refer to Fig. 2.2.

${ }^{b}$ Refer to Fig. 2.1 for assembly cell locations.

'Refer to Table 2.2 for material definitions.

${ }^{d}$ Refer to Table 2.3 for state definitions.

'Parameter definitions given in Sect. 2.5. 


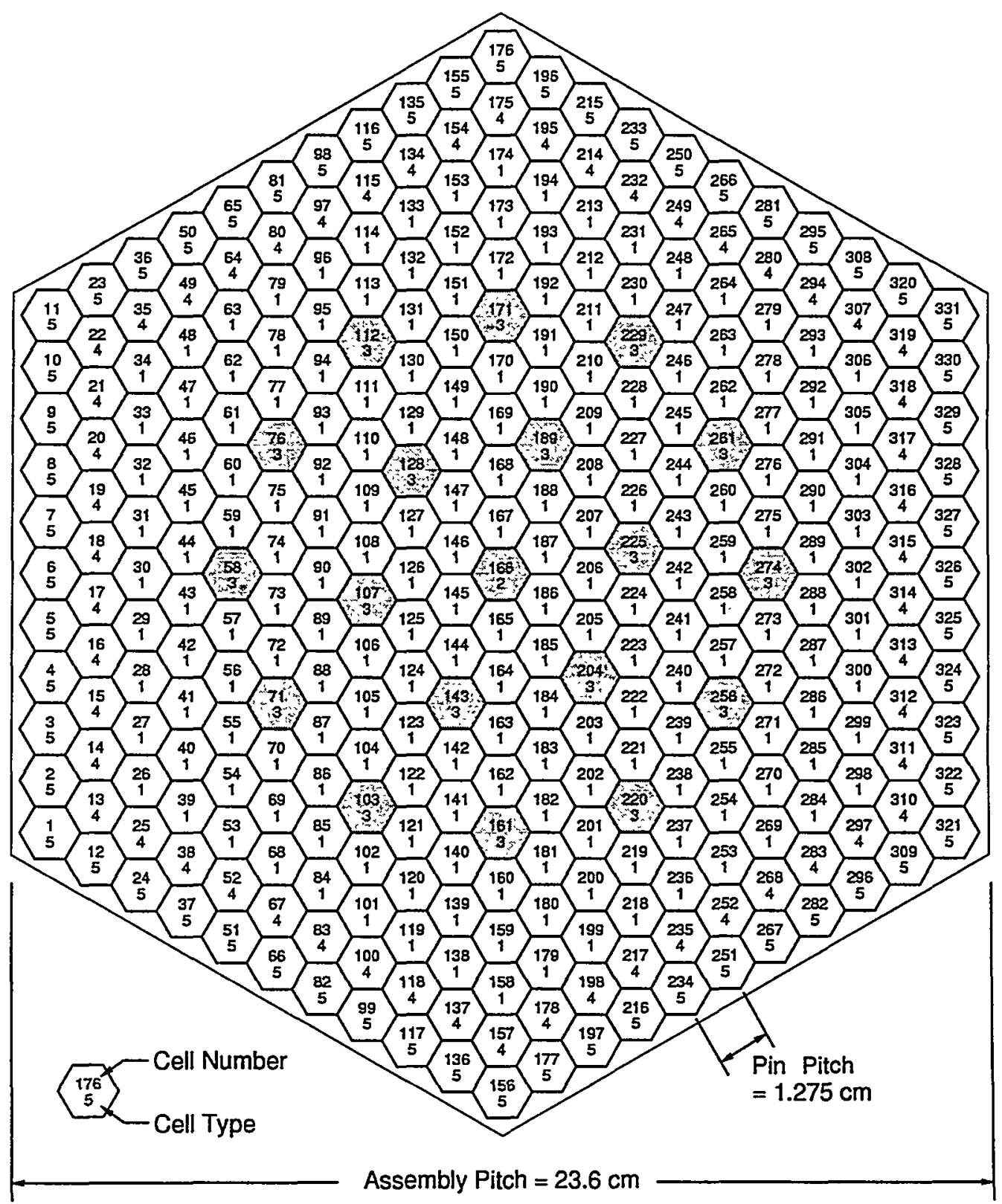

Fig. 2.1. Diagram of K331 assembly design showing cell locations and types. 


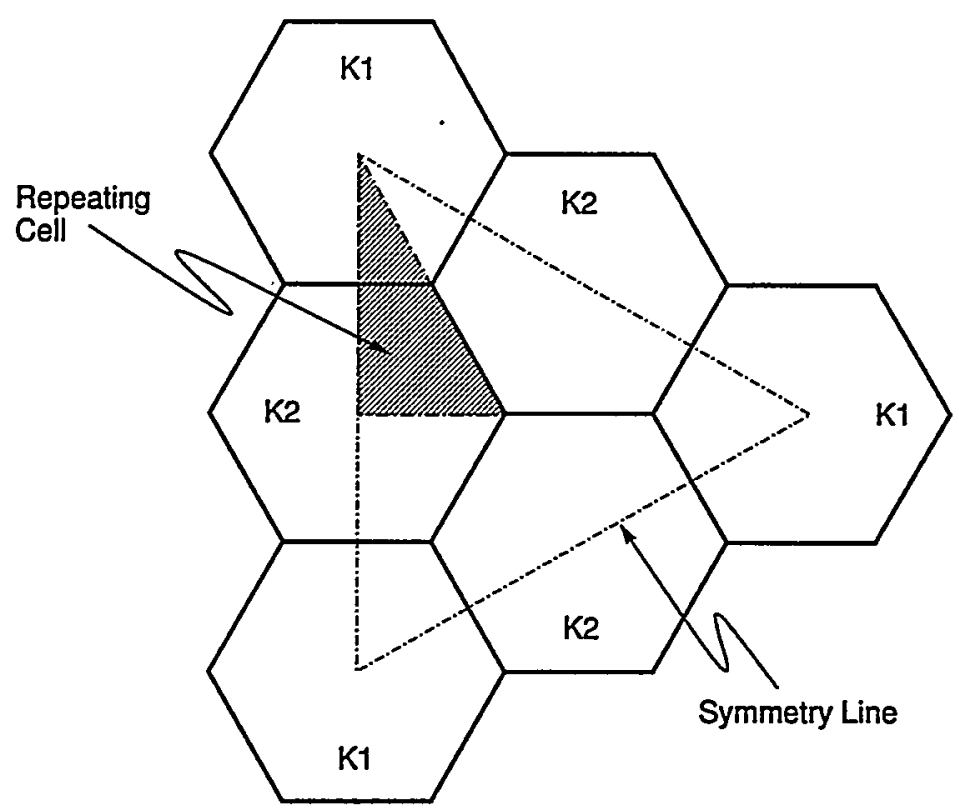

$\mathrm{K} 1, \mathrm{~K} 2=$ Assembly Type

Fig. 2.2. Geometry of MK2 multiassembly structure. 
Page Intentionally Blank 


\section{MODELING AND METHODS}

\subsection{HELIOS DESCRIPTION}

The benchmark calculations were performed with the HELIOS code system. HELIOS is a two-dimensional (2-D) fuel assembly analysis code developed by Studsvik Scandpower, Inc. The neutron and gamma transport calculations are based on current coupling of space elements, which are internally treated by collision probabilities. The resonance treatment is based on the subgroup method. Further details can be found in Refs. 2 and 3. The HELIOS system consists of the AURORA input processor, HELIOS calculation module, ZENITH output processor, and ORION geometry viewer.

\subsection{HELIOS MODELING OPTIONS}

HELIOS has several options that can be used to control the resonance processing, cell coupling order, collision probability meshing, and iterative solution. HELIOS assumes default values for these parameters, which generally provide the best solution in a reasonable amount of computer time. The values used in these calculations are as follows:

Coupling parameter:

Resonance option: Critical spectrum:

Relaxation factors:
4 (4 azimuthal angular segments, 1 polar segment)

4 (separate groups for ${ }^{238} \mathrm{U}$ and all other isotopes)

Default option to use critical spectrum for depletion and output disabled $\omega 3$ (thermal iteration relaxation factor) and $\omega 4$ (deep thermal iteration relaxation factor) were set to 1.0 . Several benchmark variants do not converge with the default value of 1.4 with the 89- and 190-group libraries.

\subsection{GEOMETRIC MODELING}

The benchmark problems consist of three different configurations: pin cells, assemblies, and multiple assemblies.

The pin cell geometric modeling is very simple. It consists of a single fuel pin, cladding, and moderator region with reflective boundary conditions. A diagram of the pin cell geometry, obtained from the ORION geometry viewer, is presented in Fig. 3.1. The fuel pin is modeled using two regions to capture the higher depletion at the periphery of the fuel material. The outer rim is $10 \%$ of the pin radius. The moderator is subdivided into six regions. This geometric model is used for benchmark Variants $1-4,7-10,15-18$, as described in Chap. 2.

The VVER-1000 assembly is modeled using one-sixth assembly symmetry with rotational boundary conditions. A diagram of the assembly geometry is shown in Fig. 3.2. The assembly consists of 312 fuel pins, 18 control pins or water hole locations, and a central instrumentation tube. The fuel pin model is identical to that described previously. Additional regions are included in and around control pins to model the sharp flux gradients near these pins. The assembly model is used to model the benchmark Variants 11 and 12 , which are $\mathrm{UO}_{2}$ and $\mathrm{MOX}$ assemblies, respectively.

Several of the benchmark variants represent an infinite lattice of MOX assemblies surrounded by $\mathrm{UO}_{2}$ assemblies at a ratio of $1 \mathrm{MOX}$ assembly per $3 \mathrm{UO}_{2}$ assemblies. This system of assemblies is modeled with one-twelfth of a MOX assembly with one-fourth of a $\mathrm{UO}_{2}$ assembly, as shown in Fig. 3.3. This model represents the repeating section shown in Fig. 2.1. The modeling details are the same as for the assembly model. Note, however, that in these benchmarks 
there is no central tube or control rod locations. The boundary conditions are specular mirror reflection on all surfaces.

\subsection{CROSS-SECTION DATA}

HELIOS's cross-section libraries are based on ENDF/B-VI revision 2 with ${ }^{235} \mathrm{U}$ from revision 3 . The ${ }^{238} \mathrm{U}$ resonance integral in the production libraries has been reduced by $3.4 \%$ to produce better agreement with critical experiments. Unadjusted libraries are also available but were not used for the benchmark calculations.

The HELIOS library contains data for 271 nuclides, of which 32 are actinides and 115 are FPs. No lumped FPs are used. The available actinides and FPs are given in Table 3.1.

The cross sections are available in 34-, 89-, and 190-group structures. Preliminary calculations of Variants 1,2 , and $10\left(\mathrm{UO}_{2}\right.$, WG MOX, and RG MOX pin cells, respectively) were performed with each of the libraries to determine their accuracy. The comparison $k_{\text {inf }}$ values at $0 \mathrm{MWd} / \mathrm{kg}$ and $60 \mathrm{MWd} / \mathrm{kg}$ are shown in Table 3.2. The results show that both the 34- and 89group libraries agree well with the 190-group results for the $\mathrm{UO}_{2}$ fuel. However, for the WG and RG MOX, the 34-group results show rather large differences at $0 \mathrm{MWd} / \mathrm{kg}$. The 89 -group results for these cases agree well with the reference 190-group results.

The 190-group library was used for all calculations of these benchmarks to provide the highest accuracy available. Based on the library comparisons, the 89-group library was shown to produce accurate results and is recommended for the analysis of MOX fuel. The 34-group library is sufficient for $\mathrm{UO}_{2}$ analysis but should be avoided when considering MOX systems.

\subsection{KINETICS PARAMETER CALCULATIONS}

Several of the benchmark variants (V15-V20) were designed to provide benchmarking of accident conditions and kinetics parameters (delayed neutron fractions and prompt neutron lifetimes). HELIOS has the capability to compute effective delayed neutron fractions via the BONE operator in ZENITH. This option, however, uses the critical spectrum for the calculation and therefore is not consistent with the effective delayed neutron fraction that is required for this benchmark. Therefore, the VENTURE ${ }^{6}$ code system was used to compute the kinetics parameters.

VENTURE is a reactor analysis system developed at ORNL. The transport method is based on finite-difference diffusion theory, and several modules are available for fuel depletion, fuel management, and perturbation analyses.

The calculational procedure for the kinetics parameters consists of transferring the homogenized cross section from HELIOS to VENTURE with the recently developed PEGASUS module. VENTURE is then used to compute the effective delayed neutron fraction and prompt neutron lifetime using an infinite, homogenous model. The effective delayed neutron fraction is computed as the real delayed neutron fraction from HELIOS multiplied by the ratio of the multiplication factor with the total (prompt and delayed) fission spectrum to the multiplication factor computed with the delayed neutron spectrum. This method of computing the effective delayed neutron fraction as the ratio of two eigenvalues is a common approximation of the rigorous adjoint-weighting method. The ratio of multiplication factors is obtained from two VENTURE eigenvalue calculations. The prompt neutron lifetime is computed as part of the VENTURE adjoint calculation. 
Table 3.1. Actinides and FPs in HELIOS library

\begin{tabular}{|c|c|c|c|c|c|c|c|}
\hline $\begin{array}{l}{ }^{233} \mathrm{Th} \\
{ }^{235} \mathrm{U} \\
{ }^{238} \mathrm{Pu} \\
{ }^{242} \mathrm{Cm}\end{array}$ & $\begin{array}{l}{ }^{242} \mathrm{Th} \\
{ }^{236} \mathrm{U} \\
{ }^{239} \mathrm{Pu} \\
{ }^{243} \mathrm{Cm}\end{array}$ & $\begin{array}{l}{ }^{231} \mathrm{~Pa} \\
{ }^{237} \mathrm{U} \\
{ }^{240} \mathrm{Pu} \\
{ }^{244} \mathrm{Cm}\end{array}$ & $\begin{array}{l}{ }^{232} \mathrm{~Pa} \\
{ }^{238} \mathrm{U} \\
{ }^{241} \mathrm{Pu} \\
{ }^{245} \mathrm{Cm}\end{array}$ & $\begin{array}{l}{ }^{233} \mathrm{~Pa} \\
{ }^{237} \mathrm{~Np} \\
{ }^{242} \mathrm{Pu} \\
{ }^{246} \mathrm{Cm}\end{array}$ & $\begin{array}{l}{ }^{234} \mathrm{~Pa} \\
{ }^{238} \mathrm{~Np} \\
{ }^{241} \mathrm{Am} \\
{ }^{232} \mathrm{U}\end{array}$ & $\begin{array}{l}{ }^{239} \mathrm{~Np} \\
{ }^{242} \mathrm{Am} \\
{ }^{233} \mathrm{U} \\
{ }^{236} \mathrm{U}\end{array}$ & $\begin{array}{l}{ }^{242 m} \mathrm{Am} \\
{ }^{234} \mathrm{U}\end{array}$ \\
\hline${ }^{81} \mathrm{Rr}_{\mathrm{r}}$ & ${ }^{83} \mathrm{Kr}$ & ${ }^{85} \mathrm{Kr}$ & ${ }^{89} \mathrm{Cr}$ & $89 y$ & ${ }^{90} \mathrm{Cr}$ & $91 \mathrm{Y}$ & 937. \\
\hline${ }^{96} \mathrm{Zr}$ & ${ }^{95} \mathrm{Zr}$ & ${ }^{95} \mathrm{Nb}$ & ${ }^{95} \mathrm{Mo}$ & ${ }^{96} \mathrm{Mo}$ & ${ }^{97} \mathrm{Mo}$ & ${ }^{98} \mathrm{Mo}$ & ${ }^{99} \mathrm{Mo}$ \\
\hline${ }^{99} \mathrm{Tc}$ & ${ }^{100} \mathrm{Ru}$ & ${ }^{101} \mathrm{Ru}$ & ${ }^{102} \mathrm{Ru}$ & ${ }^{103} \mathrm{Ru}$ & ${ }^{104} \mathrm{Ru}$ & ${ }^{105} \mathrm{Ru}$ & ${ }^{106} \mathrm{Ru}$ \\
\hline${ }^{106} \mathrm{Pd}$ & ${ }^{105} \mathrm{Rh}$ & ${ }^{105} \mathrm{Pd}$ & ${ }^{103} \mathrm{Rh}$ & ${ }^{104} \mathrm{Pd}$ & ${ }^{107} \mathrm{Pd}$ & ${ }^{108} \mathrm{Pd}$ & ${ }^{109} \mathrm{Ag}$ \\
\hline${ }^{110} \mathrm{Cd}$ & ${ }^{111} \mathrm{Cd}$ & ${ }^{110} \mathrm{Ag}$ & ${ }^{111} \mathrm{Ag}$ & ${ }^{125} \mathrm{Sb}$ & ${ }^{127} \mathrm{Sb}$ & ${ }^{127} \mathrm{I}$ & ${ }^{127 \mathrm{~m}} \mathrm{Te}$ \\
\hline${ }^{129 \mathrm{~m}} \mathrm{Te}$ & ${ }^{129} \mathrm{I}$ & ${ }^{132} \mathrm{Te}$ & ${ }^{132} \mathrm{Xe}$ & ${ }^{131}{ }_{I}$ & ${ }^{131} \mathrm{Xe}$ & ${ }^{135} \mathrm{I}$ & ${ }^{135} \mathrm{Xe}$ \\
\hline${ }^{136} \mathrm{Xe}$ & ${ }^{137} \mathrm{Cs}$ & ${ }^{137} \mathrm{Ba}$ & ${ }^{135} \mathrm{Cs}$ & ${ }^{136} \mathrm{Cs}$ & ${ }^{133} \mathrm{Xe}$ & ${ }^{134} \mathrm{Xe}$ & ${ }^{133} \mathrm{Cs}$ \\
\hline${ }^{134} \mathrm{Cs}$ & ${ }^{134} \mathrm{Ba}$ & $\begin{array}{l}{ }^{140} \mathrm{Ba} \\
\end{array}$ & ${ }^{140} \mathrm{La}$ & ${ }^{139} \mathrm{La}$ & ${ }^{142} \mathrm{Ce}$ & ${ }^{143} \mathrm{Ce}$ & ${ }^{143} \mathrm{Ce}$ \\
\hline${ }^{144} \mathrm{Ce}$ & ${ }^{147} \mathrm{Nd}$ & ${ }^{148} \mathrm{Nd}$ & ${ }^{149} \mathrm{Pm}$ & ${ }^{150} \mathrm{Sm}$ & ${ }^{151} \mathrm{Sm}$ & ${ }^{152} \mathrm{Sm}$ & ${ }^{153} \mathrm{Sm}$ \\
\hline${ }^{154} \mathrm{Sm}$ & ${ }^{153} \mathrm{Eu}$ & ${ }^{154} \mathrm{Eu}$ & ${ }^{155} \mathrm{Eu}$ & ${ }^{156} \mathrm{Eu}$ & ${ }^{157} \mathrm{Eu}$ & ${ }^{157} \mathrm{Gd}$ & ${ }^{156} \mathrm{Gd}$ \\
\hline${ }^{155} \mathrm{Gd}$ & ${ }^{154} \mathrm{Gd}$ & ${ }^{151} \mathrm{EU}$ & ${ }^{149} \mathrm{Sm}$ & ${ }^{147} \mathrm{Pm}$ & ${ }^{148} \mathrm{Pm}$ & ${ }^{148} \mathrm{Sm}$ & ${ }^{148 \mathrm{~m}} \mathrm{Pm}$ \\
\hline${ }^{147} \mathrm{Sm}$ & ${ }^{150} \mathrm{Nd}$ & ${ }^{151} \mathrm{Pm}$ & ${ }^{158} \mathrm{Gd}$ & ${ }^{159} \mathrm{~Tb}$ & ${ }^{160} \mathrm{~Tb}$ & ${ }^{161} \mathrm{~Tb}$ & ${ }^{161} \mathrm{Dy}$ \\
\hline $\begin{array}{l}{ }^{162} \mathrm{Dy} \\
{ }^{115} \mathrm{In}\end{array}$ & ${ }^{163} \mathrm{Dy}$ & $\begin{array}{l}164 \mathrm{Dy} \\
{ }^{90} \mathrm{Y}\end{array}$ & ${ }^{165} \mathrm{Ho}$ & ${ }^{160} \mathrm{Dy}$ & ${ }^{160} \mathrm{Gd}$ & ${ }^{100} \mathrm{Mo}$ & ${ }^{113} \mathrm{Cd}$ \\
\hline
\end{tabular}

Table 3.2. Comparison of $\mathbf{k}_{\text {in }}$ values from calculations using the 34-, 89-, and 190-group libraries

\begin{tabular}{|c|c|c|c|c|c|c|c|}
\hline \multirow{3}{*}{ Variant } & \multirow{3}{*}{ State } & \multicolumn{3}{|c|}{ Burnup $=0 \mathrm{MWd} / \mathrm{kg}$} & \multicolumn{3}{|c|}{ Burnup $=60 \mathrm{MWd} / \mathrm{kg}$} \\
\hline & & \multirow{2}{*}{$\begin{array}{c}\begin{array}{c}\text { Ref. } \\
\mathrm{k}_{\text {inf }}\end{array} \\
\begin{array}{c}190 \\
\text { groups }\end{array}\end{array}$} & \multicolumn{2}{|c|}{$\begin{array}{l}\text { Difference from ref. } \\
\left(\% \mathrm{dk} / \mathrm{k}^{2}\right)\end{array}$} & \multirow{2}{*}{$\begin{array}{c}\begin{array}{c}\text { Ref. } \\
\mathrm{k}_{\text {inf }}\end{array} \\
190 \\
\text { groups }\end{array}$} & \multicolumn{2}{|c|}{$\begin{array}{c}\text { Difference from ref. } \\
\left(\% \mathrm{dk} / \mathrm{k}^{2}\right)\end{array}$} \\
\hline & & & $\begin{array}{c}34 \\
\text { groups }\end{array}$ & $\begin{array}{c}89 \\
\text { groups }\end{array}$ & & $\begin{array}{c}34 \\
\text { groups }\end{array}$ & $\begin{array}{c}89 \\
\text { groups }\end{array}$ \\
\hline \multirow[t]{5}{*}{ V1 } & S1 & 1.2750 & -0.15 & -0.15 & 0.8577 & -0.26 & 0.04 \\
\hline & S3 & 1.3315 & -0.18 & -0.15 & 0.8932 & -0.21 & 0.03 \\
\hline & S4 & 1.3227 & -0.15 & $-0,15$ & 0.8894 & -0.25 & 0.01 \\
\hline & S5 & 1.3393 & -0.14 & -0.13 & 0.9046 & -0.40 & 0.00 \\
\hline & S6 & 1.3754 & -0.05 & -0.09 & 0.9321 & -0.56 & -0.20 \\
\hline \multirow[t]{5}{*}{ V2 } & S1 & 1.2188 & 0.58 & 0.15 & 0.8465 & -0.10 & 0.14 \\
\hline & S3 & 1.2481 & 0.55 & 0.15 & 0.8752 & -0.05 & 0.14 \\
\hline & S4 & 1.2383 & 0.57 & 0.15 & 0.8762 & -0.08 & 0.12 \\
\hline & S5 & 1.2583 & 0.46 & 0.13 & 0.8916 & -0.20 & 0.10 \\
\hline & S6 & 1.3309 & 0.34 & 0.12 & 0.9262 & -0.33 & 0.07 \\
\hline \multirow[t]{5}{*}{ V10 } & S1 & 1.1135 & -0.39 & -0.19 & 0.9274 & 0.22 & 0.31 \\
\hline & S3 & 1.1270 & -0.45 & -0.21 & 0.9394 & 0.19 & 0.32 \\
\hline & S4 & 1.1195 & -0.44 & -0.20 & 0.9486 & 0.21 & 0.32 \\
\hline & S5 & 1.1381 & -0.41 & -0.19 & 0.9646 & 0.19 & 0.31 \\
\hline & S6 & 1.2091 & -0.24 & -0.15 & 1.0334 & 0.33 & 0.29 \\
\hline
\end{tabular}




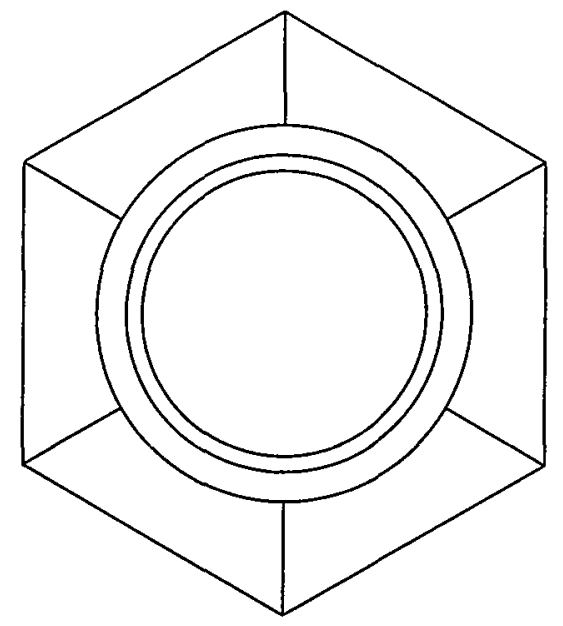

Fig. 3.1. Geometric model of pin cell variants.

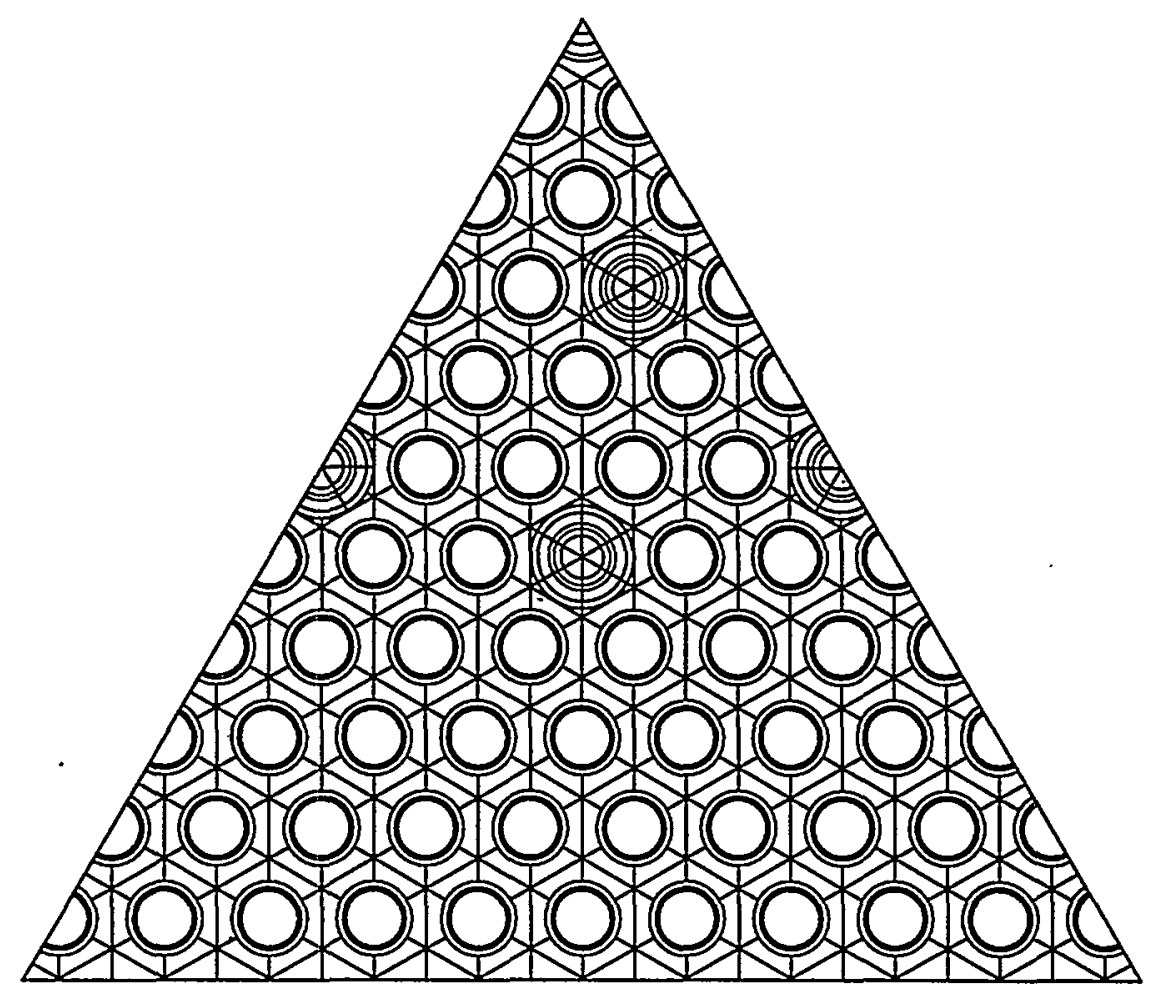

Fig. 3.2. Geometric model of assembly variants (one-sixth assembly). 


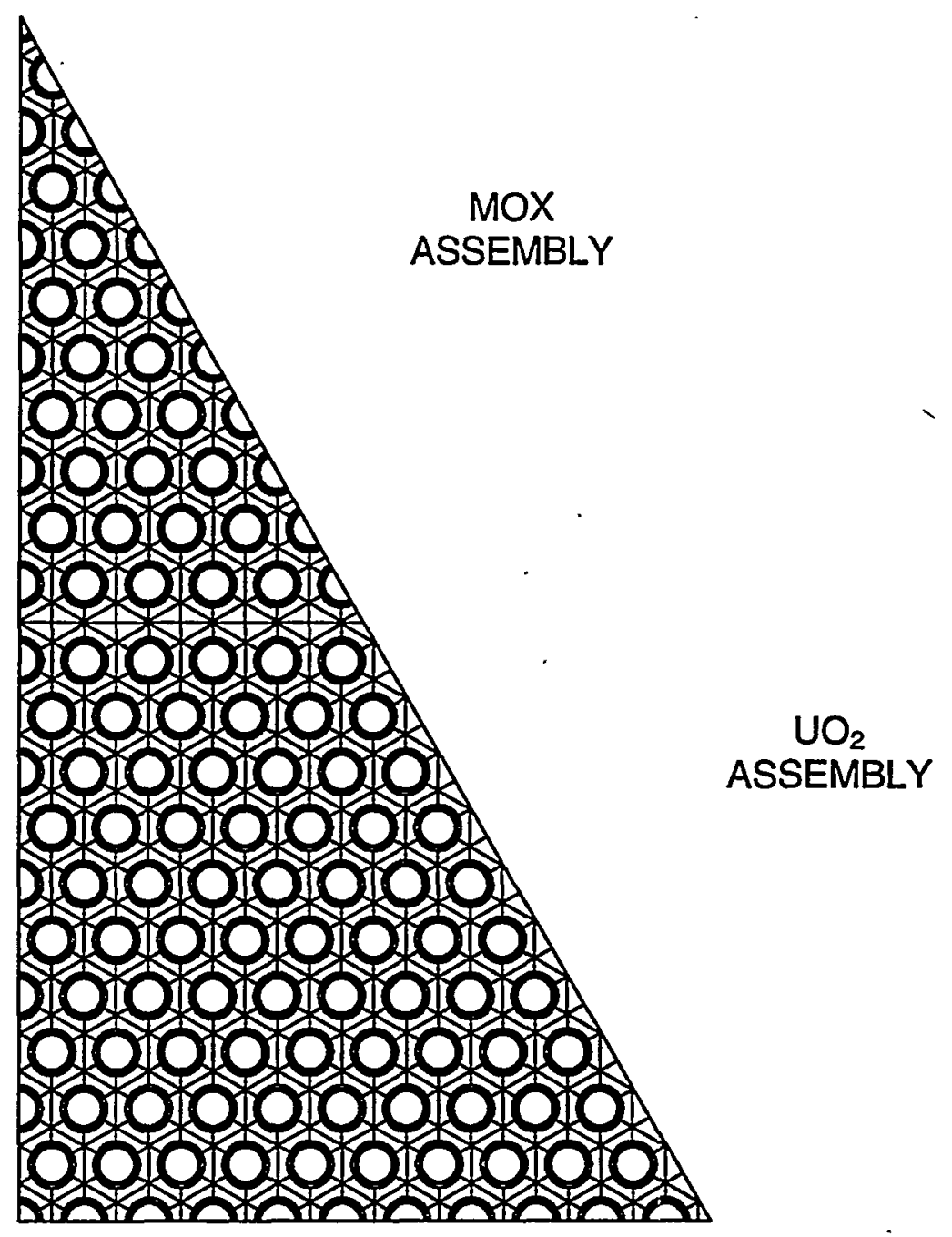

Fig. 3.3. Geometric model of multiassembly structure.

17 


\section{Page Intentionally Blank}




\section{CALCULATIONAL RESULTS}

All of the calculational results that were requested in the benchmark specifications were computed with HELIOS and are presented in Table 4.1 through Table 4.34 and Fig. 4.1 through Fig. 4.58. The quantities presented are those discussed in the problem specifications in Sect. 2.5. In particular, note that the $k_{0}$ value is the ratio of the system's total production rate to total absorption rate computed with the problem's specified buckling value and is therefore not equivalent to $k_{\text {inf. }}$ The cell relative reaction rates given in the tables (e.g., Table 4.16) are relative to the total corresponding reaction rate for the entire cell.

Table 4.1. Values of $k_{e f f}$ and $k_{0}$ at zero burnup point for Variants $1-4,7-14$, and States 1-6

\begin{tabular}{|c|c|c|c|c|c|c|c|c|c|c|c|c|}
\hline \multirow[b]{3}{*}{ Variant } & \multicolumn{12}{|c|}{ State } \\
\hline & \multicolumn{2}{|c|}{$S 1^{a}$} & \multicolumn{2}{|c|}{ S2 } & \multicolumn{2}{|c|}{$\mathrm{S}^{a}$} & \multicolumn{2}{|c|}{$S 4^{b}$} & \multicolumn{2}{|c|}{$\mathrm{S}^{b}$} & \multicolumn{2}{|c|}{$56^{b}$} \\
\hline & $\mathrm{k}_{\mathrm{eff}}$ & $\mathrm{k}_{0}$ & & $\mathrm{k}_{0}$ & $\mathrm{k}_{\mathrm{eff}}$ & $\mathrm{k}_{0}$ & $\mathrm{k}_{\text {eff }}$ & $\mathrm{k}_{0}$ & $\mathrm{k}_{\mathrm{eff}}$ & $\mathrm{k}_{0}$ & $\mathrm{k}_{\mathrm{eff}}$ & $k_{0}$ \\
\hline V1 & 1.0648 & 1.2703 & - & - & 1.1110 & 1.3259 & 1.1039 & 1.3172 & 1.1173 & 1.3339 & 1.2207 & 1.3727 \\
\hline V2 & 1.0227 & 1.2166 & - & - & 1.0469 & 1.2455 & 1.0389 & 1.2358 & 1.0551 & 1.2558 & 1.1832 & 1.3292 \\
\hline V3 & 0.9128 & 1.0915 & - & - & 0.9500 & 1.1364 & 0.9453 & 1.1305 & 0.9604 & 1.1492 & 1.0603 & 1.1946 \\
\hline V4 & 0.8602 & 1.0269 & - & - & 0.8936 & 1.0672 & 0.8890 & 1.0615 & 0.9029 & 1.0787 & 1.0005 & 1.1262 \\
\hline V7 & 1.1046 & 1.3156 & - & - & 1.1321 & 1.3487 & 1.1229 & 1.3376 & 1.1369 & 1.3550 & 1.2595 & 1.4160 \\
\hline V8 & 0.9240 & 1.1005 & - & - & 0.9690 & 1.1547 & 0.9646 & 1.1492 & 0.9800 & 1.1682 & 1.0831 & 1.2167 \\
\hline V9 & 1.3228 & 1.5676 & - & - & 1.3543 & 1.6052 & 1.3425 & 1.5911 & 1.3593 & 1.6117 & 1.4713 & 1.6484 \\
\hline V10 & 0.9593 & 1.1154 & - & - & 0.9707 & 1.1287 & 0.9643 & 1.1212 & 0.9799 & 1.1399 & 1.0910 & 1.2093 \\
\hline V11 & 1.0791 & 1.2854 & 0.8681 & 1.0312 & 1.1351 & 1.3528 & 1.1203 & 1.3347 & 1.1326 & 1.3500 & 1.2226 & 1.3713 \\
\hline V12 & 1.0483 & 1.2451 & 0.8696 & 1.0313 & 1.0788 & 1.2816 & 1.0663 & 1.2665 & 1.0812 & 1.2849 & 1.1995 & 1.3445 \\
\hline V13 & 1.0627 & 1.2652 & - & - & - & - & 1.1006 & 1.3106 & - & - & 1.2179 & 1.3672 \\
\hline V14 & 1.2726 & 1.2726 & - & - & - & - & - & - & - & - & 1.3681 & 1.3681 \\
\hline
\end{tabular}


Table 4.2. Values of $k_{\mathrm{eff}}, \mathrm{k}_{0}$, and migration area vs burnup for Variants 1,2 , and 10-12, State 1

\begin{tabular}{|c|c|c|c|c|c|c|c|c|c|c|c|c|c|c|c|}
\hline \multirow[b]{2}{*}{$\begin{array}{c}\text { Burnup } \\
\text { (MWd/kg) }\end{array}$} & \multicolumn{3}{|c|}{ Variant 1} & \multicolumn{3}{|c|}{ Variant 2} & \multicolumn{3}{|c|}{ Variant 10} & \multicolumn{3}{|c|}{ Variant 11} & \multicolumn{3}{|c|}{ Variant 12} \\
\hline & $k_{\text {eff }}$ & $\mathrm{k}_{0}$ & $\begin{array}{c}\mathrm{M}^{2} \\
\left(\mathrm{~cm}^{2}\right)\end{array}$ & $k_{\text {eff }}$ & $\mathrm{k}_{0}$ & $\begin{array}{c}\mathrm{M}^{2} \\
\left(\mathrm{~cm}^{2}\right) \\
\end{array}$ & $k_{\text {eff }}$ & $\mathrm{k}_{0}$ & $\begin{array}{c}\mathrm{M}^{2} \\
\left(\mathrm{~cm}^{2}\right) \\
\end{array}$ & $k_{\text {eff }}$ & $\mathrm{k}_{0}$ & $\begin{array}{c}\mathrm{M}^{2} \\
\left(\mathrm{~cm}^{2}\right) \\
\end{array}$ & $k_{\text {eff }}$ & $\mathrm{k}_{0}$ & $\begin{array}{c}\mathrm{M}^{2} \\
\left(\mathrm{~cm}^{2}\right) \\
\end{array}$ \\
\hline 0 & 1.0648 & 1.2703 & 66.66 & 1.0227 & 1.2166 & 64.17 & 0.9593 & 1.1154 & 53.28 & 1.0791 & 1.2854 & 66.08 & 1.0483 & 1.2451 & 63.84 \\
\hline 2 & 1.0477 & 1.2498 & 66.36 & 0.9854 & 1.1721 & 63.62 & 0.9416 & 1.0947 & 53.08 & 1.0638 & 1.2671 & 65.86 & 1.0103 & 1.1998 & 63.32 \\
\hline 4 & 1.0300 & 1.2287 & 66.07 & 0.9657 & 1.1485 & 63.37 & 0.9339 & 1.0858 & 53.02 & 1.0467 & 1.2468 & 65.61 & 0.9898 & 1.1755 & 63.09 \\
\hline 6 & 1.0118 & 1.2069 & 65.78 & 0.9486 & 1.1282 & 63.16 & 0.9275 & 1.0783 & 52.96 & 1.0291 & 1.2259 & 65.36 & 0.9719 & 1.1542 & 62.90 \\
\hline 8 & 0.9942 & 1.1859 & 65.52 & 0.9331 & 1.1098 & 62.97 & 0.9215 & 1.0714 & 52.92 & 1.0118 & 1.2053 & 65.14 & 0.9555 & 1.1348 & 62.73 \\
\hline 10 & 0.9775 & 1.1660 & 65.28 & 0.9189 & 1.0929 & 62.81 & 0.9157 & 1.0647 & 52.88 & 0.9952 & 1.1855 & 64.93 & 0.9402 & 1.1167 & 62.58 \\
\hline 12 & 0.9617 & 1.1472 & 65.07 & 0.9057 & 1.0772 & 62.67 & 0.9102 & 1.0582 & 52.85 & 0.9792 & 1.1665 & 64.75 & 0.9258 & 1.0996 & 62.45 \\
\hline 14 & 0.9468 & 1.1295 & 64.88 & 0.8933 & 1.0624 & 62.53 & 0.9046 & 1.0518 & 52.82 & 0.9639 & 1.1484 & 64.58 & 0.9122 & 1.0834 & 62.32 \\
\hline 16 & 0.9328 & 1.1127 & 64.71 & 0.8815 & 1.0484 & 62.41 & 0.8992 & 1.0456 & 52.79 & 0.9492 & 1.1309 & 64.42 & 0.8991 & 1.0679 & 62.21 \\
\hline 18 & 0.9193 & 1.0968 & 64.55 & 0.8703 & 1.0351 & 62.30 & 0.8939 & 1.0394 & 52.77 & 0.9350 & 1.1141 & 64.28 & 0.8866 & 1.0531 & 62.11 \\
\hline 20 & 0.9064 & 1.0814 & 64.41 & 0.8596 & 1.0223 & 62.20 & 0.8887 & 1.0334 & 52.74 & 0.9213 & 1.0979 & 64.15 & 0.8744 & 1.0387 & 62.01 \\
\hline 22 & 0.8940 & 1.0667 & 64.27 & 0.8493 & 1.0101 & 62.10 & 0.8836 & 1.0275 & 52.72 & 0.9080 & 1.0821 & 64.03 & 0.8627 & 1.0248 & 61.93 \\
\hline 24 & 0.8821 & 1.0525 & 64.15 & 0.8395 & 0.9984 & 62.28 & 0.8785 & 1.0216 & 52.71 & 0.8950 & 1.0667 & 63.92 & 0.8513 & 1.0113 & 61.84 \\
\hline 26 & 0.8705 & 1.0388 & 64.03 & 0.8299 & 0.9871 & 61.94 & 0.8735 & 1.0158 & 52.69 & 0.8823 & 1.0517 & 63.81 & 0.8402 & 0.9982 & 62.11 \\
\hline 28 & 0.8593 & 1.0255 & 63.93 & 0.8207 & 0.9762 & 61.87 & 0.8685 & 1.0101 & 52.67 & 0.8698 & 1.0370 & 63.71 & 0.8294 & 0.9855 & 61.70 \\
\hline 30 & 0.8483 & 1.0125 & 63.84 & 0.8118 & 0.9657 & 61.80 & 0.8636 & 1.0045 & 52.66 & 0.8576 & 1.0225 & 63.62 & 0.8189 & 0.9731 & 61.64 \\
\hline 32 & 0.8375 & 0.9997 & 63.76 & 0.8032 & 0.9554 & 61.73 & 0.8588 & 0.9989 & 52.64 & 0.8456 & 1.0083 & 63.53 & 0.8088 & 0.9611 & 61.58 \\
\hline 34 & 0.8271 & 0.9874 & 63.66 & 0.7949 & 0.9456 & 61.68 & 0.8540 & 0.9934 & 52.64 & 0.8338 & 0.9944 & 63.45 & 0.7989 & 0.9494 & 61.53 \\
\hline 36 & 0.8171 & 0.9755 & 63.58 & 0.7868 & 0.9360 & 61.63 & 0.8494 & 0.9880 & 52.63 & 0.8222 & 0.9807 & 63.37 & 0.7892 & 0.9380 & 61.49 \\
\hline 38 & 0.8072 & 0.9637 & 63.49 & 0.7791 & 0.9269 & 61.58 & 0.8448 & 0.9827 & 52.62 & 0.8109 & 0.9673 & 63.29 & 0.7799 & 0.9270 & 61.45 \\
\hline 40 & 0.7976 & 0.9524 & 63.42 & 0.7716 & 0.9180 & 61.54 & 0.8403 & 0.9775 & 52.61 & 0.7998 & 0.9541 & 63.22 & 0.7709 & 0.9163 & 61.41 \\
\hline 42 & 0.7883 & 0.9414 & 63.34 & 0.7644 & 0.9094 & 61.50 & 0.8357 & 0.9723 & 52.61 & 0.7888 & 0.9412 & 63.15 & 0.7621 & 0.9060 & 61.38 \\
\hline 44 & 0.7793 & 0.9306 & 63.28 & 0.7574 & 0.9012 & 61.47 & 0.8314 & 0.9673 & 52.61 & 0.7781 & 0.9285 & 63.09 & 0.7536 & 0.8961 & 61.36 \\
\hline 46 & 0.7704 & 0.9202 & 63.21 & 0.7506 & 0.8932 & 61.44 & 0.8270 & 0.9623 & 52.61 & 0.7677 & 0.9162 & 63.03 & 0.7455 & 0.8864 & 61.33 \\
\hline 48 & 0.7619 & 0.9100 & 63.16 & 0.7441 & 0.8855 & 61.41 & 0.8226 & 0.9572 & 52.60 & 0.7575 & 0.9041 & 62.97 & 0.7376 & 0.8771 & 61.32 \\
\hline 50 & 0.7536 & 0.9002 & 63.10 & 0.7379 & 0.8781 & 61.39 & 0.8183 & 0.9523 & 52.61 & 0.7476 & 0.8924 & 62.92 & 0.7300 & 0.8682 & 61.31 \\
\hline 52 & 0.7455 & 0.8906 & 63.05 & 0.7318 & 0.8709 & 61.37 & 0.8142 & 0.9475 & 52.61 & 0.7380 & 0.8810 & 62.87 & 0.7227 & 0.8596 & 61.30 \\
\hline 54 & 0.7378 & 0.8814 & 62.99 & 0.7259 & 0.8640 & 61.36 & 0.8101 & 0.9428 & 52.61 & 0.7287 & 0.8700 & 62.83 & 0.7156 & 0.8513 & 61.29 \\
\hline 56 & 0.7303 & 0.8726 & 62.95 & 0.7203 & 0.8574 & 61.35 & 0.8060 & 0.9382 & 52.62 & 0.7197 & 0.8594 & 62.79 & 0.7089 & 0.8434 & 61.29 \\
\hline 58 & 0.7230 & 0.8639 & 62.91 & 0.7149 & 0.8511 & 61.34 & 0.8021 & 0.9336 & 52.62 & 0.7110 & 0.8491 & 62.75 & 0.7024 & 0.8358 & 61.29 \\
\hline 60 & 0.7161 & 0.8557 & 62.87 & 0.7098 & 0.8451 & 61.32 & 0.7982 & 0.9291 & 52.62 & 0.7027 & 0.8393 & 62.72 & 0.6962 & 0.8285 & 61.30 \\
\hline
\end{tabular}


Table 4.3. Values of $k_{e f f}, k_{0}$, and migration area vs burnup for Variants 1,2 , and 10-12, States 1-6

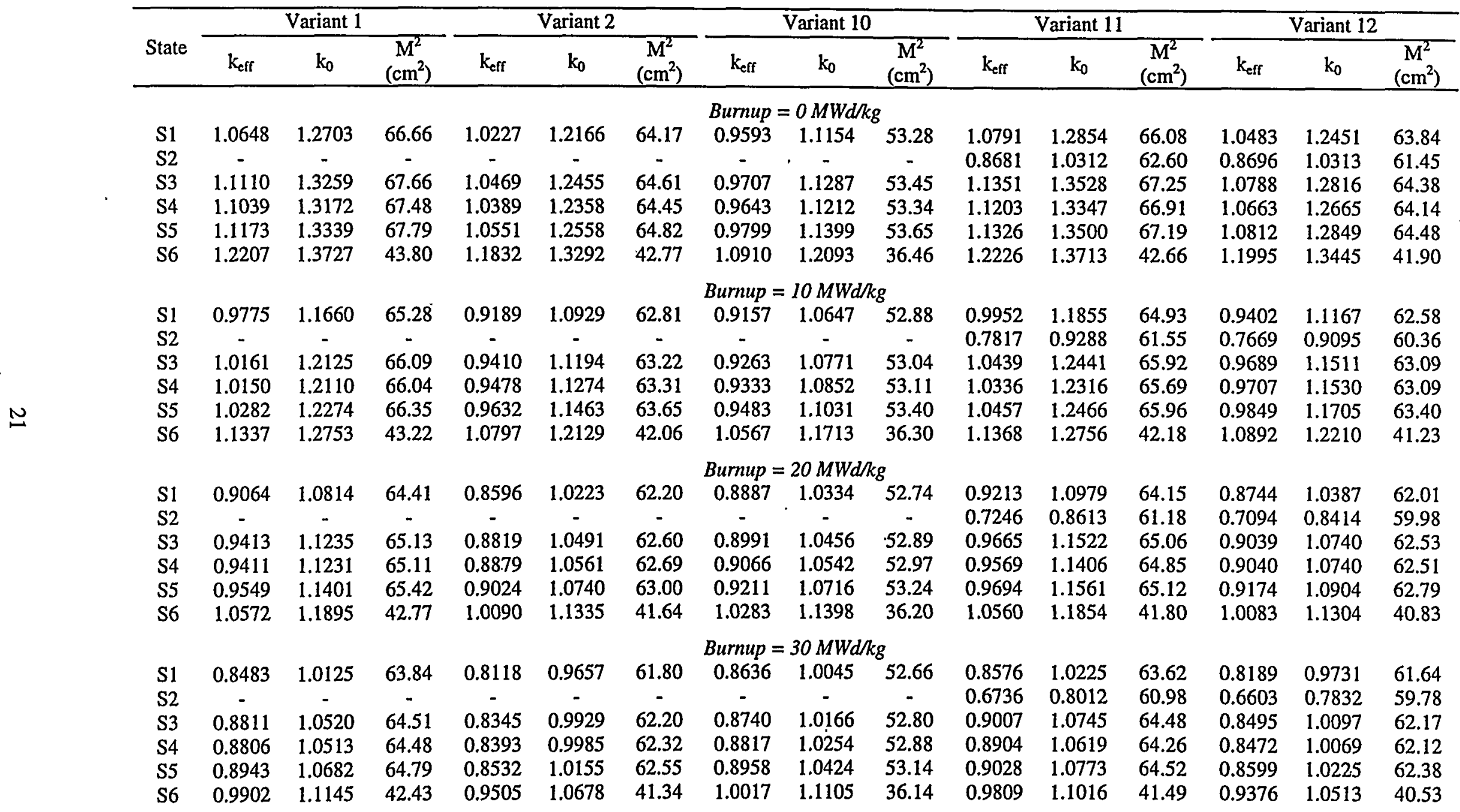


Table 4.3. (continued)

\begin{tabular}{|c|c|c|c|c|c|c|c|c|c|c|c|c|c|c|c|}
\hline \multirow[b]{2}{*}{ State } & \multicolumn{3}{|c|}{ Variant 1} & \multicolumn{3}{|c|}{ Variant 2} & \multicolumn{3}{|c|}{ Variant 10} & \multicolumn{3}{|c|}{ Variant 11} & \multicolumn{3}{|c|}{ Variant 12} \\
\hline & $k_{\text {eff }}$ & $\mathrm{k}_{0}$ & $\begin{array}{c}\mathrm{M}^{2} \\
\left(\mathrm{~cm}^{2}\right)\end{array}$ & $\mathrm{k}_{\text {eff }}$ & $\mathrm{k}_{0}$ & $\begin{array}{c}\mathrm{M}^{2} \\
\left(\mathrm{~cm}^{2}\right)\end{array}$ & $k_{\text {eff }}$ & $\mathrm{k}_{0}$ & $\begin{array}{c}\mathrm{M}^{2} \\
\left(\mathrm{~cm}^{2}\right)\end{array}$ & $k_{\text {eff }}$ & $\mathrm{k}_{0}$ & $\begin{array}{c}\mathrm{M}^{2} \\
\left(\mathrm{~cm}^{2}\right)\end{array}$ & $k_{\text {eff }}$ & $\mathrm{k}_{0}$ & $\begin{array}{c}\mathrm{M}^{2} \\
\left(\mathrm{~cm}^{2}\right) \\
\end{array}$ \\
\hline \multicolumn{16}{|c|}{ Burmup $=40 \mathrm{MWd} / \mathrm{kg}$} \\
\hline S1 & 0.7976 & 0.9524 & 63.42 & 0.7716 & 0.9180 & 61.54 & 0.8403 & 0.9775 & 52.61 & 0.7998 & 0.9541 & 63.22 & 0.7709 & 0.9163 & 61.41 \\
\hline$S 2$ & - & - & - & - & - & - & - & - & - & 0.6267 & 0.7459 & 60.88 & 0.6175 & 0.7327 & 59.71 \\
\hline S3 & 0.8288 & 0.9900 & 64.04 & 0.7947 & 0.9457 & 61.94 & 0.8505 & 0.9895 & 52.75 & 0.8414 & 1.0043 & 64.04 & 0.8025 & 0.9542 & 61.96 \\
\hline S4 & 0.8275 & 0.9883 & 64.00 & 0.7981 & 0.9497 & 61.98 & 0.8583 & 0.9985 & 52.84 & 0.8297 & 0.9901 & 63.79 & 0.7976 & 0.9483 & 61.86 \\
\hline S5 & 0.8409 & 1.0049 & 64.29 & 0.8115 & 0.9661 & 62.26 & 0.8721 & 1.0151 & 53.09 & 0.8417 & 1.0049 & 64.05 & 0.8097 & 0.9632 & 62.10 \\
\hline S6 & 0.9289 & 1.0459 & 42.16 & 0.9005 & 1.0118 & 41.14 & 0.9768 & 1.0830 & 36.09 & 0.9090 & 1.0212 & 41.22 & 0.8751 & 0.9815 & 40.32 \\
\hline \multicolumn{16}{|c|}{ Burnup $=50 \mathrm{MWd} / \mathrm{kg}$} \\
\hline S1 & 0.7536 & 0.9002 & 63.10 & 0.7379 & 0.8781 & 61.39 & 0.8183 & 0.9523 & 52.61 & 0.7476 & 0.8924 & 62.92 & 0.7300 & 0.8682 & 61.31 \\
\hline$S 2$ & & & & & & & & & & 0.5846 & 0.6961 & 60.90 & 0.5812 & 0.6900 & 59.77 \\
\hline S3 & 0.7835 & 0.9363 & 63.68 & 0.7613 & 0.9062 & 61.78 & 0.8285 & 0.9642 & 52.75 & 0.7881 & 0.9413 & 63.70 & 0.7625 & 0.9073 & 61.86 \\
\hline S4 & 0.7813 & 0.9335 & 63.63 & 0.7633 & 0.9085 & 61.81 & 0.8364 & 0.9733 & 52.83 & 0.7749 & 0.9252 & 63.43 & 0.7552 & 0.8984 & 61.72 \\
\hline S5 & 0.7942 & 0.9494 & 63.90 & 0.7763 & 0.9245 & 62.07 & 0.8500 & 0.9896 & 53.07 & 0.7863 & 0.9393 & 63.67 & 0.7668 & 0.9126 & 61.95 \\
\hline S6 & 0.8738 & 0.9841 & 41.94 & 0.8582 & 0.9645 & 41.01 & 0.9534 & 1.0573 & 36.08 & 0.8419 & 0.9463 & 41.02 & 0.8217 & 0.9218 & 40.21 \\
\hline \multicolumn{16}{|c|}{ Burnup $=60 \mathrm{MWd} / \mathrm{kg}$} \\
\hline S1 & 0.7161 & 0.8557 & 62.87 & 0.7098 & 0.8451 & 61.32 & 0.7982 & 0.9291 & 52.62 & 0.7027 & 0.8393 & 62.72 & 0.6962 & 0.8285 & 61.30 \\
\hline $\mathrm{S} 2$ & - & - & - & - & - & - & - & - & - & 0.5485 & 0.6534 & 61.01 & 0.5515 & 0.6550 & 59.92 \\
\hline \$3 & 0.7450 & 0.8906 & 63.41 & 0.7334 & 0.8734 & 61.72 & 0.8083 & 0.9410 & 52.77 & 0.7421 & 0.8868 & 63.46 & 0.7295 & 0.8686 & 61.86 \\
\hline S4 & 0.7420 & 0.8868 & 63.34 & 0.7342 & 0.8743 & 61.73 & 0.8161 & 0.9501 & 52.85 & 0.7277 & 0.8693 & 63.17 & 0.7200 & 0.8571 & 61.69 \\
\hline S5 & 0.7544 & 0.9021 & 63.60 & 0.7469 & 0.8898 & 61.97 & 0.8295 & 0.9661 & 53.08 & 0.7383 & 0.8825 & 63.38 & 0.7311 & 0.8707 & 61.89 \\
\hline S6 & 0.8262 & 0.9307 & 41.79 & 0.8229 & 0.9249 & 40.94 & 0.9318 & 1.0335 & 36.08 & 0.7831 & 0.8805 & 40.89 & 0.7775 & 0.8726 & 40.18 \\
\hline
\end{tabular}


Table 4.4. Cell macroscopic cross sections, flux, and flux ratios vs burnup for Variants 1-4 and 7-10, State 1

\begin{tabular}{|c|c|c|c|c|c|c|c|}
\hline $\begin{array}{c}\text { Burnup } \\
(\mathrm{MWd} / \mathrm{kg})\end{array}$ & $\begin{array}{c}\Sigma_{\mathrm{a}} \\
\left(\mathrm{cm}^{-1}\right)\end{array}$ & $\begin{array}{c}\Sigma_{\mathrm{f}} \\
\left(\mathrm{cm}^{-1}\right)\end{array}$ & $\begin{array}{c}v \Sigma_{\mathrm{f}} \\
\left(\mathrm{cm}^{-1}\right)\end{array}$ & $\begin{array}{c}\phi_{\text {cell }} \\
\left(\mathrm{cm}^{-2} \cdot \mathrm{s}^{-1}\right)\end{array}$ & $\phi_{\text {fued }} / \phi_{\text {cell }}$ & $\phi_{\text {clad }} / \phi_{\text {cell }}$ & $\phi_{\text {mod }} / \phi_{\text {cell }}$ \\
\hline \multicolumn{8}{|c|}{ Variant 1} \\
\hline 0 & $2.066 \mathrm{E}-2$ & $1.068 \mathrm{E}-2$ & $2.624 \mathrm{E}-2$ & $3.109 \mathrm{E}+14$ & 0.9974 & 0.9990 & 1.0019 \\
\hline 10 & $2.096 \mathrm{E}-2$ & $9.581 \mathrm{E}-3$ & $2.445 \mathrm{E}-2$ & $3.434 E+14$ & 0.9973 & 0.9989 & 1.0020 \\
\hline 20 & $2.107 \mathrm{E}-2$ & $8.709 \mathrm{E}-3$ & $2.279 \mathrm{E}-2$ & $3.752 E+14$ & 0.9971 & 0.9988 & 1.0021 \\
\hline 30 & $2.110 \mathrm{E}-2$ & $8.001 E-3$ & $2.136 \mathrm{E}-2$ & $4.061 E+14$ & 0.9968 & 0.9989 & 1.0023 \\
\hline 40 & $2.110 \mathrm{E}-2$ & $7.400 \mathrm{E}-3$ & $2.010 \mathrm{E}-2$ & $4.371 E+14$ & 0.9965 & 0.9990 & 1.0025 \\
\hline 50 & $2.110 \mathrm{E}-2$ & $6.891 \mathrm{E}-3$ & $1.899 \mathrm{E}-2$ & $4.677 E+14$ & 0.9966 & 0.9992 & 1.0023 \\
\hline 60 & $2.110 \mathrm{E}-2$ & $6.472 \mathrm{E}-3$ & $1.806 \mathrm{E}-2$ & $4.966 \mathrm{E}+14$ & 0.9967 & 0.9993 & 1.0023 \\
\hline \multicolumn{8}{|c|}{ Variant 2} \\
\hline 0 & $2.199 \mathrm{E}-2$ & $9.365 \mathrm{E}-3$ & $2.675 \mathrm{E}-2$ & $3.410 \mathrm{E}+14$ & 0.9950 & 0.9998 & 1.0032 \\
\hline .10 & $2.200 \mathrm{E}-2$ & $8.396 \mathrm{E}-3$ & $2.402 \mathrm{E}-2$ & $3.797 E+14$ & 0.9951 & 0.9997 & 1.0032 \\
\hline 20 & $2.194 \mathrm{E}-2$ & $7.818 \mathrm{E}-3$ & $2.241 \mathrm{E}-2$ & $4.074 E+14$ & 0.9952 & 0.9997 & 1.0031 \\
\hline 30 & $2.186 \mathrm{E}-2$ & $7.346 \mathrm{E}-3$ & $2.110 \mathrm{E}-2$ & $4.332 \mathrm{E}+14^{\circ}$ & 0.9953 & 0.9997 & 1.0030 \\
\hline 40 & $2.179 \mathrm{E}-2$ & $6.947 \mathrm{E}-3$ & $1.999 \mathrm{E}-2$ & $4.579 \mathrm{E}+14$ & 0.9954 & 0.9998 & 1.0029 \\
\hline 50 & $2.171 \mathrm{E}-2$ & $6.613 E-3$ & $1.906 \mathrm{E}-2$ & $4.810 \mathrm{E}+14$ & 0.9958 & 0.9996 & 1.0027 \\
\hline 60 & $2.164 \mathrm{E}-2$ & $6.338 \mathrm{E}-3$ & $1.829 \mathrm{E}-2$ & $5.020 \mathrm{E}+14$ & 0.9961 & 0.9997 & 1.0025 \\
\hline \multicolumn{8}{|c|}{ Variant 3} \\
\hline 0 & $2.083 \mathrm{E}-2$ & $8.524 \mathrm{E}-3$ & $2.273 \mathrm{E}-2$ & $3.813 \mathrm{E}+14$ & 0.9969 & 0.9990 & 1.0022 \\
\hline \multicolumn{8}{|c|}{ Variant 4} \\
\hline 0 & $2.108 \mathrm{E}-2$ & $8.116 \mathrm{E}-3$ & $2.165 \mathrm{E}-2$ & $4.005 E+14$ & 0.9967 & 0.9991 & 1.0023 \\
\hline \multicolumn{8}{|c|}{ Variant 7} \\
\hline 0 & $2.171 \mathrm{E}-2$ & $1.000 \mathrm{E}-2$ & $2.856 \mathrm{E}-2$ & $3.193 E+14$ & 0.9951 & 0.9996 & 1.0032 \\
\hline \multicolumn{8}{|c|}{ Variant 8} \\
\hline 0 & $2.054 \mathrm{E}-2$ & $9.187 \mathrm{E}-3$ & $2.261 \mathrm{E}-2$ & $3.612 E+14$ & 0.9967 & 0.9995 & 1.0022 \\
\hline \multicolumn{8}{|c|}{ Variant 9} \\
\hline 0 & $2.213 \mathrm{E}-2$ & $1.185 \mathrm{E}-2$ & $3.469 \mathrm{E}-2$ & $2.667 E+14$ & 0.9955 & 0.9994 & 1.0030 \\
\hline \multicolumn{8}{|c|}{ Variant 10} \\
\hline 0 & $2.458 \mathrm{E}-2$ & $9.522 \mathrm{E}-3$ & $2.742 \mathrm{E}-2$ & $3.346 E+14$ & 0.9937 & 1.0002 & 1.0039 \\
\hline 10 & $2.461 E-2$ & $9.086 \mathrm{E}-3$ & $2.620 \mathrm{E}-2$ & $3.505 E+14$ & 0.9938 & 1.0001 & 1.0039 \\
\hline 20 & $2.460 \mathrm{E}-2$ & $8.805 E-3$ & $2.542 \mathrm{E}-2$ & $3.615 E+14$ & 0.9938 & 1.0001 & 1.0038 \\
\hline 30 & $2.457 \mathrm{E}-2$ & $8.542 \mathrm{E}-3$ & $2.469 \mathrm{E}-2$ & $3: 725 E+14$ & 0.9939 & 1.0001 & 1.0038 \\
\hline 40 & $2.455 \mathrm{E}-2$ & $8.295 \mathrm{E}-3$ & $2.399 \mathrm{E}-2$ & $3.836 \mathrm{E}+14$ & 0.9940 & 1.0001 & 1.0037 \\
\hline 50 & $2.451 \mathrm{E}-2$ & $8.061 \mathrm{E}-3$ & $2.334 \mathrm{E}-2$ & $3.948 E+14$ & 0.9939 & 1.0004 & 1.0037 \\
\hline 60 & $2.447 \mathrm{E}-2$ & $7.844 \mathrm{E}-3$ & $2.274 \mathrm{E}-2$ & $4.057 \mathrm{E}+14$ & 0.9941 & 1.0004 & 1.0036 \\
\hline
\end{tabular}


Table 4.5. Cell macroscopic cross sections, flux, and flux ratios vs burnup for Variant 11, States 1 and 2

\begin{tabular}{|c|c|c|c|c|c|c|c|c|}
\hline State & $\begin{array}{c}\text { Burnup } \\
\text { (MWd/kg) }\end{array}$ & $\begin{array}{c}\Sigma_{\mathrm{a}} \\
\left(\mathrm{cm}^{-1}\right)\end{array}$ & $\begin{array}{c}\Sigma_{\mathrm{f}} \\
\left(\mathrm{cm}^{-1}\right)\end{array}$ & $\begin{array}{c}v \Sigma_{\mathrm{f}} \\
\left(\mathrm{cm}^{-1}\right) \\
\end{array}$ & $\begin{array}{c}\phi_{\text {cell }} \\
\left(\mathrm{cm}^{-2} \cdot \mathrm{s}^{-1}\right)\end{array}$ & $\phi_{\text {fuel }} / \phi_{\text {cell }}$ & $\phi_{\text {clad }} / \phi_{c e l l}$ & $\phi_{\text {mod }} / \phi_{\text {cell }}$ \\
\hline S1 & $\begin{array}{r}0 \\
10 \\
20 \\
30 \\
40 \\
50 \\
60\end{array}$ & $\begin{array}{l}2.376 \mathrm{E}-2 \\
2.407 \mathrm{E}-2 \\
2.409 \mathrm{E}-2 \\
2.398 \mathrm{E}-2 \\
2.383 \mathrm{E}-2 \\
2.370 \mathrm{E}-2 \\
2.361 \mathrm{E}-2\end{array}$ & $\begin{array}{l}1.265 \mathrm{E}-2 \\
1.134 \mathrm{E}-2 \\
1.019 \mathrm{E}-2 \\
9.203 \mathrm{E}-3 \\
8.344 \mathrm{E}-3 \\
7.617 \mathrm{E}-3 \\
7.036 \mathrm{E}-3\end{array}$ & $\begin{array}{c}\text { Pin } 1 \\
3.103 \mathrm{E}-2 \\
2.884 \mathrm{E}-2 \\
2.659 \mathrm{E}-2 \\
2.453 \mathrm{E}-2 \\
2.266 \mathrm{E}-2 \\
2.103 \mathrm{E}-2 \\
1.970 \mathrm{E}-2\end{array}$ & $\begin{array}{l}2.795 E+14 \\
3.075 E+14 \\
3.372 E+14 \\
3.679 E+14 \\
4.008 E+14 \\
4.351 E+14 \\
4.689 E+14\end{array}$ & $\begin{array}{l}1.0040 \\
1.0037 \\
1.0035 \\
1.0033 \\
1.0032 \\
1.0031 \\
1.0031\end{array}$ & $\begin{array}{l}1.0004 \\
1.0005 \\
1.0006 \\
1.0006 \\
1.0006 \\
1.0007 \\
1.0007\end{array}$ & $\begin{array}{l}0.9974 \\
0.9975 \\
0.9977 \\
0.9978 \\
0.9978 \\
0.9979 \\
0.9979\end{array}$ \\
\hline$S 2$ & $\begin{array}{r}0 \\
10 \\
20 \\
30 \\
40 \\
50 \\
60\end{array}$ & $\begin{array}{l}2.227 \mathrm{E}-2 \\
2.264 \mathrm{E}-2 \\
2.269 \mathrm{E}-2 \\
2.259 \mathrm{E}-2 \\
2.245 \mathrm{E}-2 \\
2.231 \mathrm{E}-2 \\
2.221 \mathrm{E}-2\end{array}$ & $\begin{array}{l}1.171 \mathrm{E}-2 \\
1.054 \mathrm{E}-2 \\
9.488 \mathrm{E}-3 \\
8.570 \mathrm{E}-3 \\
7.764 \mathrm{E}-3 \\
7.081 \mathrm{E}-3 \\
6.534 \mathrm{E}-3\end{array}$ & $\begin{array}{l}2.876 \mathrm{E}-2 \\
2.685 \mathrm{E}-2 \\
2.478 \mathrm{E}-2 \\
2.285 \mathrm{E}-2 \\
2.109 \mathrm{E}-2 \\
1.955 \mathrm{E}-2 \\
1.829 \mathrm{E}-2\end{array}$ & $\begin{array}{l}3.772 E+14 \\
4.123 E+14 \\
4.514 E+14 \\
4.930 E+14 \\
5.379 E+14 \\
5.848 E+14 \\
6.309 E+14\end{array}$ & $\begin{array}{l}1.0081 \\
1.0077 \\
1.0074 \\
1.0072 \\
1.0070 \\
1.0069 \\
1.0069\end{array}$ & $\begin{array}{l}1.0007 \\
1.0008 \\
1.0008 \\
1.0009 \\
1.0010 \\
1.0010 \\
1.0010\end{array}$ & $\begin{array}{l}0.9948 \\
0.9950 \\
0.9952 \\
0.9953 \\
0.9954 \\
0.9954 \\
0.9954\end{array}$ \\
\hline S1 & $\begin{array}{r}0 \\
10 \\
20 \\
30 \\
40 \\
50 \\
60\end{array}$ & $\begin{array}{l}2.181 \mathrm{E}-2 \\
2.207 \mathrm{E}-2 \\
2.215 \mathrm{E}-2 \\
2.215 \mathrm{E}-2 \\
2.211 \mathrm{E}-2 \\
2.205 \mathrm{E}-2 \\
2.199 \mathrm{E}-2\end{array}$ & $\begin{array}{l}1.154 \mathrm{E}-2 \\
1.042 \mathrm{E}-2 \\
9.482 \mathrm{E}-3 \\
8.683 \mathrm{E}-3 \\
7.970 \mathrm{E}-3 \\
7.336 \mathrm{E}-3 \\
6.797 \mathrm{E}-3\end{array}$ & $\begin{array}{l}P \text { in } 29 \\
2.833 \mathrm{E}-2 \\
2.648 \mathrm{E}-2 \\
2.468 \mathrm{E}-2 \\
2.306 \mathrm{E}-2 \\
2.154 \mathrm{E}-2 \\
2.014 \mathrm{E}-2 \\
1.892 \mathrm{E}-2\end{array}$ & $\begin{array}{l}2.774 \mathrm{E}+14 \\
3.054 \mathrm{E}+14 \\
3.348 \mathrm{E}+14 \\
3.655 \mathrm{E}+14 \\
3.982 \mathrm{E}+14 \\
4.324 \mathrm{E}+14 \\
4.661 \mathrm{E}+14\end{array}$ & $\begin{array}{l}1.0037 \\
1.0035 \\
1.0034 \\
1.0033 \\
1.0033 \\
1.0033 \\
1.0033\end{array}$ & $\begin{array}{l}1.0003 \\
1.0004 \\
1.0004 \\
1.0005 \\
1.0005 \\
1.0005 \\
1.0005\end{array}$ & $\begin{array}{l}0.9976 \\
0.9977 \\
0.9978 \\
0.9978 \\
0.9978 \\
0.9978 \\
0.9978\end{array}$ \\
\hline S2 & $\begin{array}{r}0 \\
10 \\
20 \\
30 \\
40 \\
50 \\
60\end{array}$ & $\begin{array}{l}1.904 \mathrm{E}-2 \\
1.934 \mathrm{E}-2 \\
1.945 \mathrm{E}-2 \\
1.945 \mathrm{E}-2 \\
1.941 \mathrm{E}-2 \\
1.935 \mathrm{E}-2 \\
1.929 \mathrm{E}-2\end{array}$ & $\begin{array}{l}9.726 \mathrm{E}-3 \\
8.824 \mathrm{E}-3 \\
8.035 \mathrm{E}-3 \\
7.349 \mathrm{E}-3 \\
6.733 \mathrm{E}-3 \\
6.185 \mathrm{E}-3 \\
5.720 \mathrm{E}-3\end{array}$ & $\begin{array}{l}2.394 \mathrm{E}-2 \\
2.246 \mathrm{E}-2 \\
2.094 \mathrm{E}-2 \\
1.953 \mathrm{E}-2 \\
1.820 \mathrm{E}-2 \\
1.699 \mathrm{E}-2 \\
1.593 \mathrm{E}-2\end{array}$ & $\begin{array}{l}3.527 \mathrm{E}+14 \\
3.862 \mathrm{E}+14 \\
4.232 \mathrm{E}+14 \\
4.624 \mathrm{E}+14 \\
5.045 \mathrm{E}+14 \\
5.484 \mathrm{E}+14 \\
5.915 \mathrm{E}+14\end{array}$ & $\begin{array}{l}1.0062 \\
1.0060 \\
1.0058 \\
1.0058 \\
1.0058 \\
1.0057 \\
1.0057\end{array}$ & $\begin{array}{l}1.0003 \\
1.0004 \\
1.0004 \\
1.0005 \\
1.0005 \\
1.0006 \\
1.0006\end{array}$ & $\begin{array}{l}0.9960 \\
0.9962 \\
0.9962 \\
0.9963 \\
0.9963 \\
0.9963 \\
0.9963\end{array}$ \\
\hline S1 & $\begin{array}{r}0 \\
10 \\
20 \\
30 . \\
40 \\
50 \\
60\end{array}$ & $\begin{array}{l}2.291 \mathrm{E}-2 \\
2.320 \mathrm{E}-2 \\
2.325 \mathrm{E}-2 \\
2.319 \mathrm{E}-2 \\
2.309 \mathrm{E}-2 \\
2.298 \mathrm{E}-2 \\
2.290 \mathrm{E}-2\end{array}$ & $\begin{array}{l}1.217 \mathrm{E}-2 \\
1.095 \mathrm{E}-2 \\
9.893 \mathrm{E}-3 \\
8.986 \mathrm{E}-3 \\
8.186 \mathrm{E}-3 \\
7.493 \mathrm{E}-3 \\
6.924 \mathrm{E}-3\end{array}$ & $\begin{array}{l}\text { Pin } 72 \\
2.988 \mathrm{E}-2 \\
2.784 \mathrm{E}-2 \\
2.579 \mathrm{E}-2 \\
2.391 \mathrm{E}-2 \\
2.218 \mathrm{E}-2 \\
2.064 \mathrm{E}-2 \\
1.934 \mathrm{E}-2\end{array}$ & $\begin{array}{l}2.790 \mathrm{E}+14 \\
3.071 \mathrm{E}+14 \\
3.367 \mathrm{E}+14 \\
3.674 \mathrm{E}+14 \\
4.002 \mathrm{E}+14 \\
4.345 \mathrm{E}+14 \\
4.682 \mathrm{E}+14\end{array}$ & $\begin{array}{l}1.0038 \\
1.0035 \\
1.0033 \\
1.0032 \\
1.0031 \\
1.0030 \\
1.0030\end{array}$ & $\begin{array}{l}1.0003 \\
1.0003 \\
1.0004 \\
1.0004 \\
1.0005 \\
1.0005 \\
1.0005\end{array}$ & $\begin{array}{l}0.9976 \\
0.9977 \\
0.9978 \\
0.9979 \\
0.9979 \\
0.9980 \\
0.9980\end{array}$ \\
\hline$S 2$ & $\begin{array}{r}0 \\
10 \\
20 \\
30 \\
40 \\
50 \\
60\end{array}$ & $\begin{array}{l}1.623 \mathrm{E}-2 \\
1.651 \mathrm{E}-2 \\
1.660 \mathrm{E}-2 \\
1.658 \mathrm{E}-2 \\
1.653 \mathrm{E}-2 \\
1.648 \mathrm{E}-2 \\
1.644 \mathrm{E}-2 \\
\end{array}$ & $\begin{array}{l}7.815 \mathrm{E}-3 \\
7.057 \mathrm{E}-3 \\
6.376 \mathrm{E}-3 \\
5.781 \mathrm{E}-3 \\
5.255 \mathrm{E}-3 \\
4.802 \mathrm{E}-3 \\
4.433 \mathrm{E}-3 \\
\end{array}$ & $\begin{array}{l}1.929 \mathrm{E}-2 \\
1.802 \mathrm{E}-2 \\
1.667 \mathrm{E}-2 \\
1.542 \mathrm{E}-2 \\
1.426 \mathrm{E}-2 \\
1.324 \mathrm{E}-2 \\
1.238 \mathrm{E}-2 \\
\end{array}$ & $\begin{array}{l}3.182 \mathrm{E}+14 \\
3.496 \mathrm{E}+14 \\
3.834 \mathrm{E}+14 \\
4.188 \mathrm{E}+14 \\
4.565 \mathrm{E}+14 \\
4.957 \mathrm{E}+14 \\
5.343 \mathrm{E}+14 \\
\end{array}$ & $\begin{array}{l}1.0037 \\
1.0034 \\
1.0032 \\
1.0030 \\
1.0029 \\
1.0028 \\
1.0028 \\
\end{array}$ & $\begin{array}{l}1.0002 \\
1.0003 \\
1.0003 \\
1.0004 \\
1.0004 \\
1.0004 \\
1.0004 \\
\end{array}$ & $\begin{array}{l}0.9977 \\
0.9978 \\
0.9979 \\
0.9980 \\
0.9981 \\
0.9981 \\
0.9981 \\
\end{array}$ \\
\hline
\end{tabular}


Table 4.6. Cell macroscopic cross sections, flux, and flux ratios vs burnup for Variant 12, States 1 and 2

\begin{tabular}{|c|c|c|c|c|c|c|c|c|}
\hline State & $\begin{array}{c}\text { Burnup } \\
\text { (MWd/kg) }\end{array}$ & $\begin{array}{c}\begin{array}{c}\Sigma_{\mathrm{a}} \\
\left(\mathrm{cm}^{-1}\right)\end{array} \\
\end{array}$ & $\begin{array}{c}\Sigma_{\mathrm{f}} \\
\left(\mathrm{cm}^{-1}\right)\end{array}$ & $\begin{array}{c}v \Sigma_{\mathrm{f}} \\
\left(\mathrm{cm}^{-1}\right)\end{array}$ & $\begin{array}{c}\phi_{\text {cell }} \\
\left(\mathrm{cm}^{-2} \cdot \mathrm{s}^{-1}\right)\end{array}$ & $\phi_{\text {fruel }} / \phi_{\text {cell }}$ & $\phi_{\text {clad }} / \phi_{\text {cell }}$ & $\phi_{\text {mod }} / \phi_{\text {cell }}$ \\
\hline S1 & $\begin{array}{r}0 \\
10 \\
20 \\
30 \\
40 \\
50 \\
60\end{array}$ & $\begin{array}{l}2.638 \mathrm{E}-2 \\
2.603 \mathrm{E}-2 \\
2.554 \mathrm{E}-2 \\
2.509 \mathrm{E}-2 \\
2.471 \mathrm{E}-2 \\
2.441 \mathrm{E}-2 \\
2.418 \mathrm{E}-2\end{array}$ & $\begin{array}{l}1.173 \mathrm{E}-2 \\
1.023 \mathrm{E}-2 \\
9.231 \mathrm{E}-3 \\
8.421 \mathrm{E}-3 \\
7.774 \mathrm{E}-3 \\
7.269 \mathrm{E}-3 \\
6.878 \mathrm{E}-3\end{array}$ & $\begin{array}{c}\text { Pin } 1 \\
3.351 \mathrm{E}-2 \\
2.926 \mathrm{E}-2 \\
2.647 \mathrm{E}-2 \\
2.420 \mathrm{E}-2 \\
2.239 \mathrm{E}-2 \\
2.097 \mathrm{E}-2 \\
1.986 \mathrm{E}-2\end{array}$ & $\begin{array}{l}3.027 \mathrm{E}+14 \\
3.383 \mathrm{E}+14 \\
3.661 \mathrm{E}+14 \\
3.941 \mathrm{E}+14 \\
4.224 \mathrm{E}+14 \\
4.499 \mathrm{E}+14 \\
4.754 \mathrm{E}+14\end{array}$ & $\begin{array}{l}1.0025 \\
1.0024 \\
1.0023 \\
1.0023 \\
1.0024 \\
1.0025 \\
1.0026\end{array}$ & $\begin{array}{l}1.0011 \\
1.0011 \\
1.0010 \\
1.0010 \\
1.0009 \\
1.0009 \\
1.0009\end{array}$ & $\begin{array}{l}0.9981 \\
0.9982 \\
0.9983 \\
0.9983 \\
0.9983 \\
0.9982 \\
0.9982\end{array}$ \\
\hline S2 & $\begin{array}{r}0 \\
10 \\
20 \\
30 \\
40 \\
50 \\
60\end{array}$ & $\begin{array}{l}2.511 \mathrm{E}-2 \\
2.479 \mathrm{E}-2 \\
2.431 \mathrm{E}-2 \\
2.385 \mathrm{E}-2 \\
2.345 \mathrm{E}-2 \\
2.314 \mathrm{E}-2 \\
2.288 \mathrm{E}-2\end{array}$ & $\begin{array}{l}1.111 \mathrm{E}-2 \\
9.693 \mathrm{E}-3 \\
8.735 \mathrm{E}-3 \\
7.951 \mathrm{E}-3 \\
7.321 \mathrm{E}-3 \\
6.827 \mathrm{E}-3 \\
6.442 \mathrm{E}-3\end{array}$ & $\begin{array}{l}3.174 \mathrm{E}-2 \\
2.773 \mathrm{E}-2 \\
2.505 \mathrm{E}-2 \\
2.285 \mathrm{E}-2 \\
2.108 \mathrm{E}-2 \\
1.969 \mathrm{E}-2 \\
1.859 \mathrm{E}-2\end{array}$ & $\begin{array}{l}3.906 \mathrm{E}+14 \\
4.373 \mathrm{E}+14 \\
4.758 \mathrm{E}+14 \\
5.152 \mathrm{E}+14 \\
5.554 \mathrm{E}+14 \\
5.947 \mathrm{E}+14 \\
6.315 \mathrm{E}+14\end{array}$ & $\begin{array}{l}1.0063 \\
1.0061 \\
1.0060 \\
1.0060 \\
1.0060 \\
1.0062 \\
1.0063\end{array}$ & $\begin{array}{l}1.0013 \\
1.0013 \\
1.0013 \\
1.0013 \\
1.0012 \\
1.0012 \\
1.0012\end{array}$ & $\begin{array}{l}0.9957 \\
0.9959 \\
0.9959 \\
0.9960 \\
0.9959 \\
0.9958 \\
0.9958\end{array}$ \\
\hline S1 & $\begin{array}{r}0 \\
10 \\
20 \\
30 \\
40 \\
50 \\
60\end{array}$ & $\begin{array}{l}2.308 \mathrm{E}-2 \\
2.313 \mathrm{E}-2 \\
2.312 \mathrm{E}-2 \\
2.306 \mathrm{E}-2 \\
2.295 \mathrm{E}-2 \\
2.281 \mathrm{E}-2 \\
2.266 \mathrm{E}-2\end{array}$ & $\begin{array}{l}1.006 \mathrm{E}-2 \\
9.094 \mathrm{E}-3 \\
8.490 \mathrm{E}-3 \\
7.955 \mathrm{E}-3 \\
7.465 \mathrm{E}-3 \\
7.030 \mathrm{E}-3 \\
6.660 \mathrm{E}-3\end{array}$ & $\begin{array}{l}\text { Pin } 29 \\
2.874 \mathrm{E}-2 \\
2.602 \mathrm{E}-2 \\
2.433 \mathrm{E}-2 \\
2.284 \mathrm{E}-2 \\
2.148 \mathrm{E}-2 \\
2.026 \mathrm{E}-2 \\
1.921 \mathrm{E}-2\end{array}$ & $\begin{array}{l}2.999 E+14 \\
3.354 E+14 \\
3.632 E+14 \\
3.912 E+14 \\
4.194 E+14 \\
4.468 E+14 \\
4.722 E+14\end{array}$ & $\begin{array}{l}1.0019 \\
1.0021 \\
1.0023 \\
1.0025 \\
1.0026 \\
1.0027 \\
1.0028\end{array}$ & $\begin{array}{l}1.0009 \\
1.0008 \\
1.0008 \\
1.0008 \\
1.0008 \\
1.0007 \\
1.0007\end{array}$ & $\begin{array}{l}0.9986 \\
0.9985 \\
0.9984 \\
0.9983 \\
0.9982 \\
0.9981 \\
0.9981\end{array}$ \\
\hline S2 & $\begin{array}{r}0 \\
10 \\
20 \\
30 \\
40 \\
50 \\
60\end{array}$ & $\begin{array}{l}2.062 \mathrm{E}-2 \\
2.066 \mathrm{E}-2 \\
2.060 \mathrm{E}-2 \\
2.049 \mathrm{E}-2 \\
2.034 \mathrm{E}-2 \\
2.017 \mathrm{E}-2 \\
2.001 \mathrm{E}-2\end{array}$ & $\begin{array}{l}8.785 E-3 \\
7.927 E-3 \\
7.363 E-3 \\
6.862 E-3 \\
6.406 E-3 \\
6.002 E-3 \\
5.664 E-3\end{array}$ & $\begin{array}{l}2.510 \mathrm{E}-2 \\
2.267 \mathrm{E}-2 \\
2.110 \mathrm{E}-2 \\
1.970 \mathrm{E}-2 \\
1.842 \mathrm{E}-2 \\
1.729 \mathrm{E}-2 \\
1.633 \mathrm{E}-2\end{array}$ & $\begin{array}{l}3.685 \mathrm{E}+14 \\
4.129 \mathrm{E}+14 \\
4.490 \mathrm{E}+14 \\
4.858 \mathrm{E}+14 \\
5.232 \mathrm{E}+14 \\
5.596 \mathrm{E}+14 \\
5.936 \mathrm{E}+14\end{array}$ & $\begin{array}{l}1.0044 \\
1.0045 \\
1.0047 \\
1.0049 \\
1.0050 \\
1.0052 \\
1.0053\end{array}$ & $\begin{array}{l}1.0008 \\
1.0008 \\
1.0008 \\
1.0008 \\
1.0008 \\
1.0007 \\
1.0007\end{array}$ & $\begin{array}{l}0.9971 \\
0.9970 \\
0.9969 \\
0.9968 \\
0.9967 \\
0.9966 \\
0.9965\end{array}$ \\
\hline S1 & $\begin{array}{r}0 \\
10 \\
20 \\
30 \\
40 \\
50 \\
60\end{array}$ & $\begin{array}{l}2.488 \mathrm{E}-2 \\
2.475 \mathrm{E}-2 \\
2.450 \mathrm{E}-2 \\
2.423 \mathrm{E}-2 \\
2.397 \mathrm{E}-2 \\
2.373 \mathrm{E}-2 \\
2.353 \mathrm{E}-2\end{array}$ & $\begin{array}{l}1.098 \mathrm{E}-2 \\
9.738 \mathrm{E}-3 \\
8.926 \mathrm{E}-3 \\
8.236 \mathrm{E}-3 \\
7.650 \mathrm{E}-3 \\
7.166 \mathrm{E}-3 \\
6.778 \mathrm{E}-3\end{array}$ & $\begin{array}{l}\text { Pin } 72 \\
3.137 \mathrm{E}-2 \\
2.786 \mathrm{E}-2 \\
2.559 \mathrm{E}-2 \\
2.366 \mathrm{E}-2 \\
2.202 \mathrm{E}-2 \\
2.066 \mathrm{E}-2 \\
1.956 \mathrm{E}-2\end{array}$ & $\begin{array}{l}3.019 \mathrm{E}+14 \\
3.375 \mathrm{E}+14 \\
3.654 \mathrm{E}+14 \\
3.934 \mathrm{E}+14 \\
4.216 \mathrm{E}+14 \\
4.491 \mathrm{E}+14 \\
4.747 \mathrm{E}+14\end{array}$ & $\begin{array}{l}1.0022 \\
1.0022 \\
1.0022 \\
1.0023 \\
1.0023 \\
1.0024 \\
1.0026\end{array}$ & $\begin{array}{l}1.0009 \\
1.0009 \\
1.0008 \\
1.0008 \\
1.0008 \\
1.0007 \\
1.0007\end{array}$ & $\begin{array}{l}0.9984 \\
0.9984 \\
0.9984 \\
0.9984 \\
0.9983 \\
0.9983 \\
0.9982\end{array}$ \\
\hline S2 & $\begin{array}{r}0 \\
10 \\
20 \\
30 \\
40 \\
50 \\
60\end{array}$ & $\begin{array}{l}1.787 \mathrm{E}-2 \\
1.782 \mathrm{E}-2 \\
1.766 \mathrm{E}-2 \\
1.747 \mathrm{E}-2 \\
1.729 \mathrm{E}-2 \\
1.712 \mathrm{E}-2 \\
1.698 \mathrm{E}-2\end{array}$ & $\begin{array}{l}7.297 \mathrm{E}-3 \\
6.466 \mathrm{E}-3 \\
5.895 \mathrm{E}-3 \\
5.408 \mathrm{E}-3 \\
4.996 \mathrm{E}-3 \\
4.658 \mathrm{E}-3 \\
4.388 \mathrm{E}-3\end{array}$ & $\begin{array}{l}2.084 \mathrm{E}-2 \\
1.849 \mathrm{E}-2 \\
1.689 \mathrm{E}-2 \\
1.553 \mathrm{E}-2 \\
1.437 \mathrm{E}-2 \\
1.341 \mathrm{E}-2 \\
1.265 \mathrm{E}-2\end{array}$ & $\begin{array}{l}3.402 \mathrm{E}+14 \\
3.804 \mathrm{E}+14 \\
4.125 \mathrm{E}+14 \\
4.449 \mathrm{E}+14 \\
4.777 \mathrm{E}+14 \\
5.098 \mathrm{E}+14 \\
5.394 \mathrm{E}+14\end{array}$ & $\begin{array}{l}1.0025 \\
1.0024 \\
1.0024 \\
1.0023 \\
1.0023 \\
1.0024 \\
1.0024\end{array}$ & $\begin{array}{l}1.0006 \\
1.0006 \\
1.0006 \\
1.0006 \\
1.0006 \\
1.0006 \\
1.0005\end{array}$ & $\begin{array}{l}0.9983 \\
0.9983 \\
0.9984 \\
0.9984 \\
0.9984 \\
0.9984 \\
0.9984\end{array}$ \\
\hline
\end{tabular}


Table 4.7. Isotopic concentration vs burnup for Variant 1, State 1

\begin{tabular}{|c|c|c|c|c|c|c|c|c|c|c|c|c|c|c|c|c|c|}
\hline \multirow{2}{*}{$\begin{array}{c}\text { Bumup } \\
\text { (MWd/kg) }\end{array}$} & \multicolumn{17}{|c|}{ Concentration (atoms $/ \mathrm{b} \cdot \mathrm{cm}$, in fuel) } \\
\hline & ${ }^{235} \mathrm{U}$ & ${ }^{236} \mathrm{U}$ & ${ }^{238} \mathrm{U}$ & ${ }^{238} \mathrm{Pu}$ & ${ }^{239} \mathrm{Pu}$ & ${ }^{240} \mathrm{Pu}$ & ${ }^{241} \mathrm{Pu}$ & ${ }^{242} \mathrm{Pu}$ & ${ }^{211} \mathrm{Am}$ & ${ }^{242 m} \mathrm{Am}$ & ${ }^{243} \mathrm{Am}$ & ${ }^{242} \mathrm{Cm}$ & ${ }^{243} \mathrm{Cm}$ & ${ }^{244} \mathrm{Cm}$ & ${ }^{135} \mathrm{Xe}$ & ${ }^{149} \mathrm{Sm}$ & Total FP \\
\hline 0 & $8.737 \mathrm{E}-4$ & 0.0 & $1.874 \mathrm{E}-2$ & 0.0 & 0.0 & 0.0 & 0.0 & 0.0 & 0.0 & 0.0 & 0.0 & 0.0 & 0.0 & 0.0 & $9.458 \mathrm{E}-9$ & $7.367 \mathrm{E}-8$ & 8.313E-8 \\
\hline 2 & $8.271 \mathrm{E}-4$ & $8.912 \mathrm{E}-6$ & $1.872 \mathrm{E}-2$ & $1.509 \mathrm{E}-9$ & $1.786 \mathrm{E}-5$ & $5.448 \mathrm{E}-7$ & $4.873 \mathrm{E}-8$ & $4.852 \mathrm{E}-10$ & $7.284 \mathrm{E}-11$ & $2.278 \mathrm{E}-13$ & $3.978 \mathrm{E}-12$ & $1.264 \mathrm{E}-12$ & 0.0 & 0.0 & $1.009 \mathrm{E}-8$ & $8.031 \mathrm{E}-8$ & $6.640 \mathrm{E}-5$ \\
\hline 4 & $7.832 \mathrm{E}-4$ & $1.726 \mathrm{E}-5$ & $1.870 \mathrm{E}-2$ & $9.533 \mathrm{E}-9$ & $3.424 \mathrm{E}-5$ & $1.982 \mathrm{E}-6$ & $3.591 \mathrm{E}-7$ & $7.440 \mathrm{E}-9$ & $1.115 \mathrm{E}-9$ & $6.460 \mathrm{E}-12$ & $1.299 \mathrm{E}-10$ & $3.895 \mathrm{E}-11$ & $6.427 \mathrm{E}-14$ & $2.744 \mathrm{E}-12$ & $1.022 \mathrm{E}-8$ & $8.594 \mathrm{E}-8$ & $1.326 \mathrm{E}-4$ \\
\hline 6 & $7.416 \mathrm{E}-4$ & $2.513 \mathrm{E}-5$ & $1.867 \mathrm{E}-2$ & $2.744 \mathrm{E}-8$ & $4.832 \mathrm{E}-5$ & $3.942 \mathrm{E}-6$ & $1.044 \mathrm{E}-6$ & $3.347 \mathrm{E}-8$ & $4.969 \mathrm{E}-9$ & $3.988 \mathrm{E}$ & $9.168 \mathrm{E}$ & $2.599 \mathrm{E}$ & $6.825 \mathrm{E}-13$ & 3.094E-11 & $1.030 \mathrm{E}-8$ & $9.107 \mathrm{E}-8$ & $1.991 \mathrm{E}-4$ \\
\hline 8 & $7.021 \mathrm{E}-4$ & $3.255 \mathrm{E}-5$ & $1.865 \mathrm{E}-2$ & $5.807 \mathrm{E}-8$ & $6.051 \mathrm{E}-5$ & $6.211 \mathrm{E}-6$ & $2.103 \mathrm{E}-6$ & $9.246 \mathrm{E}-8$ & $1.355 \mathrm{E}-8$ & $1.342 \mathrm{E}-10$ & $3.496 \mathrm{E}-9$ & $98 \mathrm{E}$ & $385 E-12$ & $630 \mathrm{E}-10$ & $1.034 \mathrm{E}-8$ & $9.550 \mathrm{E}-8$ & $2.658 \mathrm{E}-4$ \\
\hline 10 & $6.645 \mathrm{E}-4$ & $3.956 \mathrm{E}-5$ & $1.862 \mathrm{E}-2$ & $1.041 \mathrm{E}-7$ & $7.113 \mathrm{E}-5$ & $8.666 \mathrm{E}-6$ & $3.487 \mathrm{E}-6$ & $1.967 \mathrm{E}-7$ & $2.836 \mathrm{E}-8$ & $3.262 \mathrm{E}-10$ & $9.589 \mathrm{E}-9$ & $2.445 \mathrm{E}-9$ & $1.129 \mathrm{E}-11$ & 5.755E-10 & $1.035 \mathrm{E}-8$ & $9.931 \mathrm{E}-8$ & $3.325 \mathrm{E}-4$ \\
\hline 12 & $6.286 \mathrm{E}-4$ & $4.619 \mathrm{E}-5$ & $1.860 \mathrm{E}-2$ & $1.678 \mathrm{E}-7$ & $8.042 \mathrm{E}-5$ & $1.123 \mathrm{E}-5$ & $5.134 \mathrm{E}-6$ & $3.558 \mathrm{E}-7$ & $5.039 \mathrm{E}-8$ & $6.479 \mathrm{E}-10$ & $2.139 \mathrm{E}-8$ & $5.179 \mathrm{E}-9$ & $2.935 \mathrm{E}-11$ & $1.582 \mathrm{E}-9$ & $1.034 \mathrm{E}-8$ & $1.026 \mathrm{E}-7$ & $.993 \mathrm{E}-4$ \\
\hline 14 & $5.943 \mathrm{E}-4$ & $5.245 \mathrm{E}-5$ & $1.857 \mathrm{E}-2$ & $2.514 \mathrm{E}-7$ & $8.855 \mathrm{E}-5$ & $1.386 \mathrm{E}-5$ & $6.981 \mathrm{E}-6$ & 5.770E-7 & $8.011 \mathrm{E}-8$ & $1.123 \mathrm{E}-9$ & $4.141 \mathrm{E}-8$ & $9.536 \mathrm{E}-9$ & $6.426 \mathrm{E}-11$ & $3.664 \mathrm{E}-9$ & $1.032 \mathrm{E}-8$ & $1.053 \mathrm{E}-7$ & $4.661 \mathrm{E}-4$ \\
\hline 16 & $5.616 \mathrm{E}-4$ & $5.836 \mathrm{E}-5$ & $1.854 \mathrm{E}-2$ & $3.568 \mathrm{E}-7$ & $9.569 \mathrm{E}-5$ & $1.652 \mathrm{E}-5$ & 8.972E-6 & $8.648 \mathrm{E}-7$ & $1.175 \mathrm{E}-7$ & $1.764 \mathrm{E}-9$ & $7.236 \mathrm{E}-8$ & $1.587 \mathrm{E}-8$ & $1.243 \mathrm{E}-10$ & $7.492 \mathrm{E}-9$ & $1.028 \mathrm{E}-8$ & $1.076 \mathrm{E}-7$ & $E-4$ \\
\hline 18 & 03E-4 & $6.393 \mathrm{E}-5$ & $1.852 \mathrm{E}-2$ & $4.858 \mathrm{E}-7$ & $1.020 \mathrm{E}-4$ & $1.918 \mathrm{E}-5$ & $1.106 \mathrm{E}-5$ & 8.6 & -7 & -9 & $\mathrm{E}-7$ & $2.448 \mathrm{E}-8$ & $2.188 \mathrm{E}-10$ & $1.394 \mathrm{E}-8$ & $1.023 \mathrm{E}-8$ & $1.095 \mathrm{E}-7$ & $.998 \mathrm{E}-4$ \\
\hline 20 & $5.004 \mathrm{E}-4$ & $6.918 \mathrm{E}-5$ & $1.849 \mathrm{E}-2$ & $6.400 \mathrm{E}-7$ & $1.075 \mathrm{E}-4$ & $2.182 \mathrm{E}-5$ & 1.319E-5 & $1.650 \mathrm{E}-6$ & $2.141 \mathrm{E}-7$ & $3.551 \mathrm{E}-9$ & $1.780 \mathrm{E}-7$ & $3.558 \mathrm{E}-8$ & $3.578 \mathrm{E}-10$ & $2.407 \mathrm{E}-8$ & $1.017 \mathrm{E}-8$ & $1.111 \mathrm{E}-7$ & $6.666 \mathrm{E}-4$ \\
\hline 22 & $4.718 \mathrm{E}-4$ & $7.412 \mathrm{E}-5$ & $1.846 \mathrm{E}-2$ & 8.207E-7 & $1.123 \mathrm{E}-4$ & $2.443 \mathrm{E}-5$ & $1.535 \mathrm{E}-5$ & $2.147 \mathrm{E}-6$ & $2.718 \mathrm{E}-7$ & $4.681 \mathrm{E}-9$ & $2.579 \mathrm{E}-7$ & $4.932 \mathrm{E}-8$ & $5.516 \mathrm{E}-10$ & $3.915 \mathrm{E}-8$ & $1.010 \mathrm{E}-8$ & $1.124 \mathrm{E}-7$ & $E-4$ \\
\hline 24 & $4.445 \mathrm{E}-4$ & $7.875 \mathrm{E}-5$ & $1.843 \mathrm{E}-2$ & $1.029 \mathrm{E}-6$ & $1.165 \mathrm{E}-4$ & $2.701 \mathrm{E}-5$ & $1.750 \mathrm{E}-5$ & $2.714 \mathrm{E}-6$ & $3.348 \mathrm{E}-7$ & $5.948 \mathrm{E}-9$ & $3.591 \mathrm{E}-7$ & $6.577 \mathrm{E}-8$ & $8.102 \mathrm{E}-10$ & $6.064 \mathrm{E}-8$ & $1.002 \mathrm{E}-8$ & $1.134 \mathrm{E}-7$ & $8.003 E-4$ \\
\hline 26 & $4.184 \mathrm{E}-4$ & $8.310 \mathrm{E}-5$ & $1.840 \mathrm{E}-2$ & $1.266 \mathrm{E}-6$ & $1.202 \mathrm{E}-4$ & $2.952 \mathrm{E}-5$ & $1.962 \mathrm{E}-5$ & $3.347 \mathrm{E}-6$ & $4.021 \mathrm{E}-7$ & $7.333 \mathrm{E}-9$ & $4.836 \mathrm{E}-7$ & $8.492 \mathrm{E}-8$ & $1.143 E-9$ & $9.014 \mathrm{E}-8$ & $9.943 \mathrm{E}-9$ & $1.142 \mathrm{E}-7$ & E-4 \\
\hline 28 & $3.935 \mathrm{E}-4$ & $8.716 \mathrm{E}-5$ & $1.837 \mathrm{E}-2$ & $1.532 \mathrm{E}-6$ & $1.235 \mathrm{E}-4$ & $3.199 \mathrm{E}-5$ & $2.170 \mathrm{E}-5$ & $4.045 \mathrm{E}-6$ & $4.727 \mathrm{E}-7$ & $8.812 \mathrm{E}-9$ & $6.330 \mathrm{E}-7$ & $1.067 \mathrm{E}-7$ & $1.558 \mathrm{E}-9$ & $1.294 \mathrm{E}-7$ & $9.857 \mathrm{E}-9$ & $1.147 \mathrm{E}-7$ & $9.340 \mathrm{E}-4$ \\
\hline 30 & $3.697 \mathrm{E}-4$ & $9.094 \mathrm{E}-5$ & $1.834 \mathrm{E}-2$ & $1.828 \mathrm{E}-6$ & $1.263 \mathrm{E}-4$ & $3.438 \mathrm{E}-5$ & 2.371E-5 & $4.803 \mathrm{E}-6$ & $5.457 \mathrm{E}-7$ & $1.036 \mathrm{E}-8$ & $8.087 \mathrm{E}-7$ & $1.310 \mathrm{E}-7$ & $2.062 \mathrm{E}-9$ & $1.803 \mathrm{E}-7$ & $9.767 \mathrm{E}-9$ & $1.150 \mathrm{E}-7$ & E-3 \\
\hline 32 & $3.471 \mathrm{E}-4$ & $9.446 \mathrm{E}-5$ & $1.831 \mathrm{E}-2$ & $2.154 \mathrm{E}-6$ & $1.287 \mathrm{E}-4$ & $3.670 \mathrm{E}-5$ & $2.565 \mathrm{E}-5$ & $5.619 \mathrm{E}-6$ & $6.201 \mathrm{E}-7$ & $1.197 \mathrm{E}-8$ & $2 \mathrm{E}-6$ & $1.577 \mathrm{E}-7$ & $2.660 \mathrm{E}-9$ & $2.448 \mathrm{E}-7$ & $9.673 \mathrm{E}-9$ & $1.151 \mathrm{E}-7$ & $1: 068 \mathrm{E}-3$ \\
\hline 34 & $3.255 \mathrm{E}-4$ & $9.773 \mathrm{E}-5$ & $1.828 \mathrm{E}-2$ & $2.510 \mathrm{E}-6$ & $1.308 \mathrm{E}-4$ & 3.895E-5 & $2.751 \mathrm{E}-5$ & $6.488 \mathrm{E}-6$ & $6.950 \mathrm{E}-7$ & $1.359 \mathrm{E}-8$ & $1.243 \mathrm{E}-6$ & $1.866 \mathrm{E}-7$ & $3.357 \mathrm{E}-9$ & $3.249 \mathrm{E}-7$ & $9.578 \mathrm{E}-9$ & $1.151 \mathrm{E}-7$ & E-3 \\
\hline 36 & $3.050 \mathrm{E}-4$ & $1.007 \mathrm{E}-4$ & $1.825 \mathrm{E}-2$ & $2.895 \mathrm{E}-6$ & $1.327 \mathrm{E}-4$ & $4.112 \mathrm{E}-5$ & $2.929 \mathrm{E}-5$ & $7.408 \mathrm{E}-6$ & $7.698 \mathrm{E}-7$ & $1.523 \mathrm{E}-8$ & $1.503 \mathrm{E}-6$ & $2.173 \mathrm{E}-7$ & $4.153 \mathrm{E}-9$ & $4.225 \mathrm{E}-7$ & 9.482E-9 & $1.150 \mathrm{E}-7$ & E-3 \\
\hline 38 & $2.854 \mathrm{E}-4$ & $1.035 \mathrm{E}-4$ & $1.822 \mathrm{E}-2$ & $3.310 \mathrm{E}-6$ & $1.342 \mathrm{E}-4$ & $4.321 \mathrm{E}-5$ & 3.098E-5 & $8.373 E-6$ & $8.436 \mathrm{E}-7$ & $1.685 \mathrm{E}-8$ & $1.791 \mathrm{E}-6$ & $2.497 \mathrm{E}-7$ & $5.050 \mathrm{E}-9$ & 5.399E-7 & $9.385 \mathrm{E}-9$ & $1.148 \mathrm{E}-7$ & $1.268 \mathrm{E}-3$ \\
\hline 40 & $2.669 \mathrm{E}-4$ & $1.060 \mathrm{E}-4$ & $1.819 \mathrm{E}-2$ & $3.753 \mathrm{E}-6$ & $1.356 \mathrm{E}-4$ & $4.521 \mathrm{E}-5$ & $3.258 \mathrm{E}-5$ & $9.379 \mathrm{E}-6$ & $9.158 \mathrm{E}-7$ & $1.844 \mathrm{E}-8$ & $2.107 \mathrm{E}-6$ & 2.835E-7 & $6.044 \mathrm{E}-9$ & $6.788 \mathrm{E}-7$ & $9.289 \mathrm{E}-9$ & $1.145 \mathrm{E}-7$ & $1.335 \mathrm{E}-3$ \\
\hline 42 & $2.493 \mathrm{E}-4$ & $1.084 \mathrm{E}-4$ & $1.815 \mathrm{E}-2$ & $4.223 \mathrm{E}-6$ & $1.367 \mathrm{E}-4$ & $4.712 \mathrm{E}-5$ & $3.409 \mathrm{E}-5$ & $1.042 \mathrm{E}-5$ & $9.859 \mathrm{E}-7$ & $1.999 \mathrm{E}-8$ & $2.451 \mathrm{E}-6$ & $3.184 \mathrm{E}-7$ & $7.132 \mathrm{E}-9$ & $8.412 E-7$ & $9.192 \mathrm{E}-9$ & $1.141 \mathrm{E}-7$ & $1.402 \mathrm{E}-3$ \\
\hline 44 & $2.326 \mathrm{E}-4$ & $1.104 \mathrm{E}-4$ & $1.812 \mathrm{E}-2$ & $4.719 \mathrm{E}-6$ & $1.377 \mathrm{E}-4$ & $4.895 \mathrm{E}-5$ & $3.551 \mathrm{E}-5$ & $1.150 \mathrm{E}-5$ & $1.053 \mathrm{E}-6$ & $2.148 \mathrm{E}-8$ & $2.821 \mathrm{E}-6$ & $3.542 \mathrm{E}-7$ & $8.308 \mathrm{E}-9$ & $1.029 \mathrm{E}-6$ & $9.096 \mathrm{E}-9$ & $1.137 \mathrm{E}-7$ & $1.469 \mathrm{E}-3$ \\
\hline 46 & $2.168 \mathrm{E}-4$ & $1.123 \mathrm{E}-4$ & $1.809 \mathrm{E}-2$ & $5.240 \mathrm{E}-6$ & $1.385 \mathrm{E}-4$ & $5.070 \mathrm{E}-5$ & $3.683 \mathrm{E}-5$ & $1.261 \mathrm{E}-5$ & $1.118 \mathrm{E}-6$ & $2.291 \mathrm{E}-8$ & $3.217 \mathrm{E}-6$ & $3.904 \mathrm{E}-7$ & $9.567 \mathrm{E}-9$ & 1.244E-6 & $9.002 \mathrm{E}-9$ & $1.131 \mathrm{E}-7$ & $1.536 \mathrm{E}-3$ \\
\hline 48 & $2.019 \mathrm{E}-4$ & $1.140 \mathrm{E}-4$ & $1.805 \mathrm{E}-2$ & $5.785 \mathrm{E}-6$ & $1.392 \mathrm{E}-4$ & $5.236 \mathrm{E}-5$ & 3.807E-5 & $1.374 \mathrm{E}-5$ & $1.179 \mathrm{E}-6$ & $2.426 \mathrm{E}-8$ & $3.637 \mathrm{E}-6$ & $4.269 \mathrm{E}-7$ & $1.090 \mathrm{E}-8$ & $1.487 \mathrm{E}-6$ & $8.909 \mathrm{E}-9$ & $1.126 \mathrm{E}-7$ & $1.602 \mathrm{E}-3$ \\
\hline 50 & $1.878 \mathrm{E}-4$ & $1.154 \mathrm{E}-4$ & $1.802 \mathrm{E}-2$ & $6.350 \mathrm{E}-6$ & $1.398 \mathrm{E}-4$ & $5.394 \mathrm{E}-5$ & $3.922 \mathrm{E}-5$ & $1.489 \mathrm{E}-5$ & $1.236 \mathrm{E}-6$ & $2.553 \mathrm{E}-8$ & $4.080 \mathrm{E}-6$ & $4.635 \mathrm{E}-7$ & $1.230 \mathrm{E}-8$ & $1.760 \mathrm{E}-6$ & $8.818 \mathrm{E}-9$ & $1.120 \mathrm{E}-7$ & $1.669 \mathrm{E}-3$ \\
\hline 52 & $1.745 \mathrm{E}-4$ & $1.167 \mathrm{E}-4$ & $1.799 \mathrm{E}-2$ & $6.936 \mathrm{E}-6$ & $1.403 \mathrm{E}-4$ & $5.544 \mathrm{E}-5$ & $4.028 \mathrm{E}-5$ & $1.606 \mathrm{E}-5$ & $1.290 \mathrm{E}-6$ & $2.671 \mathrm{E}-8$ & $4.545 \mathrm{E}-6$ & $4.998 \mathrm{E}-7$ & $1.376 \mathrm{E}-8$ & $2.065 \mathrm{E}-6$ & $8.730 \mathrm{E}-9$ & $1.114 \mathrm{E}-7$ & $1.736 \mathrm{E}-3$ \\
\hline 54 & $1.620 \mathrm{E}-4$ & $1.178 \mathrm{E}-4$ & $1.795 \mathrm{E}-2$ & $7.539 \mathrm{E}-6$ & $1.407 \mathrm{E}-4$ & $5.686 \mathrm{E}-5$ & $4.127 \mathrm{E}-5$ & $1.725 \mathrm{E}-5$ & $1.340 \mathrm{E}-6$ & $2.781 \mathrm{E}-8$ & $5.028 \mathrm{E}-6$ & $5.356 \mathrm{E}-7$ & $1.526 \mathrm{E}-8$ & $2.402 \mathrm{E}-6$ & 8.645E-9 & $1.108 \mathrm{E}-7$ & $1.803 \mathrm{E}-3$ \\
\hline 56 & $1.502 \mathrm{E}-4$ & $1.187 \mathrm{E}-4$ & $1.792 \mathrm{E}-2$ & $8.157 \mathrm{E}-6$ & $1.410 \mathrm{E}-4$ & $5.819 \mathrm{E}-5$ & $4.219 \mathrm{E}-5$ & $1.845 \mathrm{E}-5$ & $1.386 \mathrm{E}-6$ & $2.882 \mathrm{E}-8$ & $5.530 \mathrm{E}-6$ & 5.707E-7 & $1.679 \mathrm{E}-8$ & $2.771 \mathrm{E}-6$ & $8.563 \mathrm{E}-9$ & $1.103 \mathrm{E}-7$ & $1.870 \mathrm{E}-3$ \\
\hline 58 & 1.392E-4 & 1.195E-4 & $1.788 \mathrm{E}-2$ & $8.789 \mathrm{E}-6$ & $1.412 \mathrm{E}-4$ & $5.945 E-5$ & $4.303 \mathrm{E}-5$ & $1.965 \mathrm{E}-5$ & $1.429 \mathrm{E}-6$ & $2.975 \mathrm{E}-8$ & $6.047 \mathrm{E}-6$ & $6.049 \mathrm{E}-7$ & $1.835 \mathrm{E}-8$ & $3.174 \mathrm{E}-6$ & 8.484E-9 & $1.097 \mathrm{E}-7$ & $1.936 \mathrm{E}-3$ \\
\hline 60 & $1.288 \mathrm{E}-4$ & $1.201 \mathrm{E}-4$ & $1.784 \mathrm{E}-2$ & $9.432 \mathrm{E}-6$ & $1.414 \mathrm{E}-4$ & $6.064 \mathrm{E}-5$ & $4.381 \mathrm{E}-5$ & $2.086 \mathrm{E}-5$ & $1.467 \mathrm{E}-6$ & $3.059 \mathrm{E}-8$ & $6.578 \mathrm{E}-6$ & $6.381 \mathrm{E}-7$ & $1.992 \mathrm{E}-8$ & $3.611 \mathrm{E}-6$ & 8.409E-9 & $1.092 \mathrm{E}-7$ & $2.003 \mathrm{E}-3$ \\
\hline
\end{tabular}


Table 4.8. Isotopic concentration vs burnup for Variant 2, State 1

\begin{tabular}{|c|c|c|c|c|c|c|c|c|c|c|c|c|c|c|c|c|c|}
\hline \multirow{2}{*}{$\begin{array}{c}\text { Burnup } \\
(\mathrm{MWd} / \mathrm{kg})\end{array}$} & \multicolumn{17}{|c|}{ Concentration (atoms $/ \mathrm{b} \cdot \mathrm{cm}$, in fuel) } \\
\hline & ${ }^{235} \mathrm{U}$ & ${ }^{236} \mathrm{U}$ & ${ }^{238} \mathrm{U}$ & ${ }^{238} \mathrm{Pu}$ & ${ }^{239} \mathrm{Pu}$ & ${ }^{240} \mathrm{Pu}$ & ${ }^{241} \mathrm{Pu}$ & ${ }^{242} \mathrm{Pu}$ & ${ }^{241} \mathrm{Am}$ & ${ }^{242 m} \mathrm{Am}$ & ${ }^{243} \mathrm{Am}$ & ${ }^{242} \mathrm{Cm}$ & ${ }^{243} \mathrm{Cm}$ & ${ }^{244} \mathrm{Cm}$ & ${ }^{135} \mathrm{Xe}$ & ${ }^{149} \mathrm{Sm}$ & Total FP \\
\hline 0 & $3.839 \mathrm{E}-5$ & 0.0 & $1.892 \mathrm{E}-2$ & 0.0 & $6.588 \mathrm{E}-4$ & $4.232 \mathrm{E}-5$ & $7.025 \mathrm{E}-6$ & 0.0 & 0.0 & 0.0 & 0.0 & 0.0 & 0.0 & 0.0 & $9.458 \mathrm{E}-9$ & $7.367 \mathrm{E}-8$ & $8.313 \mathrm{E}-8$ \\
\hline 2 & $3.703 \mathrm{E}-5$ & $3.125 \mathrm{E}-7$ & $1.889 \mathrm{E}-2$ & $1.581 \mathrm{E}-9$ & $6.255 \mathrm{E}-4$ & $5.501 \mathrm{E}-5$ & $1.266 \mathrm{E}-5$ & $2.025 \mathrm{E}-7$ & $5.941 \mathrm{E}-8$ & $2.760 \mathrm{E}-10$ & 4.472E-9 & $1.489 \mathrm{E}-9$ & $2.446 \mathrm{E}-12$ & $9.710 \mathrm{E}-11$ & $1.699 \mathrm{E}-8$ & $1.664 \mathrm{E}-7$ & $6.787 \mathrm{E}-5$ \\
\hline 4 & $3.569 \mathrm{E}-5$ & $6.180 \mathrm{E}-7$ & $1.887 \mathrm{E}-2$ & $8.735 \mathrm{E}-9$ & $5.951 E-4$ & $6.649 \mathrm{E}-5$ & $1.868 \mathrm{E}-5$ & $5.278 \mathrm{E}-7$ & $1.494 \mathrm{E}-7$ & $1.196 \mathrm{E}-9$ & $2.170 \mathrm{E}-8$ & $6.815 \mathrm{E}-9$ & $2.202 \mathrm{E}-11$ & $9.192 \mathrm{E}-10$ & $1.667 \mathrm{E}-8$ & $1.759 \mathrm{E}-7$ & $1.355 \mathrm{E}-4$ \\
\hline 6 & $3.435 \mathrm{E}-5$ & $9.153 \mathrm{E}-7$ & $1.884 \mathrm{E}-2$ & $2.275 \mathrm{E}-8$ & $5.660 \mathrm{E}-4$ & $7.690 \mathrm{E}-5$ & $2.483 \mathrm{E}-5$ & $9.834 \mathrm{E}-7$ & $2.687 \mathrm{E}-7$ & $2.843 \mathrm{E}-9$ & $5.743 \mathrm{E}-8$ & $1.709 \mathrm{E}-8$ & $8.082 \mathrm{E}-11$ & $3.560 \mathrm{E}-9$ & $1.634 \mathrm{E}-8$ & $1.777 \mathrm{E}-7$ & $2.034 \mathrm{E}-4$ \\
\hline 8 & $3.304 \mathrm{E}-5$ & $1.204 \mathrm{E}-6$ & $1.881 \mathrm{E}-2$ & $4.475 \mathrm{E}-8$ & $5.381 E-4$ & $8.634 \mathrm{E}-5$ & 3.099E-5 & $1.573 \mathrm{E}-6$ & $4.147 \mathrm{E}-7$ & $5.246 \mathrm{E}-9$ & $1.173 \mathrm{E}-7$ & $3.319 \mathrm{E}-8$ & $2.050 \mathrm{E}-10$ & $9.513 \mathrm{E}-9$ & $1.602 \mathrm{E}-8$ & $1.787 \mathrm{E}-7$ & $2.714 \mathrm{E}-4$ \\
\hline 10 & $3.174 \mathrm{E}-5$ & $1.483 \mathrm{E}-6$ & $1.878 \mathrm{E}-2$ & $7.595 \mathrm{E}-8$ & $5.115 \mathrm{E}-4$ & $9.485 \mathrm{E}-5$ & $3.704 \mathrm{E}-5$ & $2.297 \mathrm{E}-6$ & $5.843 \mathrm{E}-7$ & 8.386E-9 & $2.067 \mathrm{E}-7$ & $5.576 \mathrm{E}-8$ & $4.232 \mathrm{E}-10$ & $2.065 \mathrm{E}-8$ & $1.570 \mathrm{E}-8$ & $1.790 \mathrm{E}-7$ & $3.393 \mathrm{E}-4$ \\
\hline 12 & $3.046 \mathrm{E}-5$ & $1.753 \mathrm{E}-6$ & $1.875 \mathrm{E}-2$ & $1.176 \mathrm{E}-7$ & $4.861 \mathrm{E}-4$ & $1.025 \mathrm{E}-4$ & $4.292 \mathrm{E}-5$ & $3.153 \mathrm{E}-6$ & $7.740 \mathrm{E}-7$ & $1.221 \mathrm{E}-8$ & $3.304 \mathrm{E}-7$ & $8.525 \mathrm{E}-8$ & $7.647 \mathrm{E}-10$ & $3.920 \mathrm{E}-8$ & $1.539 \mathrm{E}-8$ & $1.789 \mathrm{E}-7$ & $4.073 \mathrm{E}-4$ \\
\hline 14 & $2.921 \mathrm{E}-5$ & $2.013 E-6$ & $1.872 \mathrm{E}-2$ & $1.709 \mathrm{E}-7$ & $4.620 \mathrm{E}-4$ & $1.093 \mathrm{E}-4$ & $4.855 \mathrm{E}-5$ & $4.140 \mathrm{E}-6$ & $9.800 \mathrm{E}-7$ & $1.662 \mathrm{E}-8$ & $4.924 \mathrm{E}-7$ & $1.219 \mathrm{E}-7$ & $1.259 \mathrm{E}-9$ & $6.766 \mathrm{E}-8$ & $1.509 \mathrm{E}-8$ & $1.784 \mathrm{E}-7$ & $4.752 \mathrm{E}-4$ \\
\hline 16 & $2.798 \mathrm{E}-5$ & $2.263 \mathrm{E}-6$ & $1.870 \mathrm{E}-2$ & $2.371 \mathrm{E}-7$ & $4.392 \mathrm{E}-4$ & $1.153 \mathrm{E}-4$ & $5.389 \mathrm{E}-5$ & $5.252 \mathrm{E}-6$ & 1.199E-6 & $2.154 \mathrm{E}-8$ & $6.962 \mathrm{E}-7$ & $1.656 \mathrm{E}-7$ & $1.933 \mathrm{E}-9$ & $1.088 \mathrm{E}-7$ & $1.479 \mathrm{E}-8$ & $1.776 \mathrm{E}-7$ & $5.431 \mathrm{E}-4$ \\
\hline 18 & $2.677 \mathrm{E}-5$ & $2.503 \mathrm{E}-6$ & $1.867 \mathrm{E}-2$ & 3.173E-7 & $4.176 \mathrm{E}-4$ & $1.206 \mathrm{E}-4$ & $5.890 \mathrm{E}-5$ & $6.484 \mathrm{E}-6$ & $1.426 \mathrm{E}-6$ & $2.683 \mathrm{E}-8$ & $9.444 \mathrm{E}-7$ & $2.163 \mathrm{E}-7$ & $2.810 \mathrm{E}-9$ & $1.656 \mathrm{E}-7$ & $1.450 \mathrm{E}-8$ & $1.765 \mathrm{E}-7$ & $6.110 \mathrm{E}-4$ \\
\hline 20 & $2.558 \mathrm{E}-5$ & $2.732 \mathrm{E}-6$ & $1.864 \mathrm{E}-2$ & $4.125 \mathrm{E}-7$ & $3.972 \mathrm{E}-4$ & $1.252 \mathrm{E}-4$ & $6.357 \mathrm{E}-5$ & $7.829 \mathrm{E}-6$ & $1.658 \mathrm{E}-6$ & $3.239 \mathrm{E}-8$ & $1.239 \mathrm{E}-6$ & $2.736 \mathrm{E}-7$ & $3.913 \mathrm{E}-9$ & $2.410 \mathrm{E}-7$ & $1.421 \mathrm{E}-8$ & $1.751 \mathrm{E}-7$ & $6.788 \mathrm{E}-4$ \\
\hline 22 & $2.443 \mathrm{E}-5$ & $2.951 \mathrm{E}-6$ & $1.861 \mathrm{E}-2$ & $5.234 \mathrm{E}-7$ & $3.781 E-4$ & $1.291 \mathrm{E}-4$ & $6.786 \mathrm{E}-5$ & $9.281 \mathrm{E}-6$ & $1.891 \mathrm{E}-6$ & $3.811 \mathrm{E}-8$ & $1.580 \mathrm{E}-6$ & 3.370E-7 & $5.257 \mathrm{E}-9$ & 3.383E-7 & $1.393 \mathrm{E}-8$ & $1.735 \mathrm{E}-7$ & $7.467 \mathrm{E}-4$ \\
\hline 24 & $2.330 \mathrm{E}-5$ & $3.160 \mathrm{E}-6$ & $1.857 \mathrm{E}-2$ & $6.508 \mathrm{E}-7$ & $3.601 E-4$ & $1.323 \mathrm{E}-4$ & $7.176 \mathrm{E}-5$ & $1.083 \mathrm{E}-5$ & $2.122 \mathrm{E}-6$ & $4.386 \mathrm{E}-8$ & $1.970 \mathrm{E}-6$ & $4.060 \mathrm{E}-7$ & $6.852 \mathrm{E}-9$ & $4.604 \mathrm{E}-7$ & $1.366 \mathrm{E}-8$ & $1.718 \mathrm{E}-7$ & $8.145 E-4$ \\
\hline 26 & $2.219 \mathrm{E}-5$ & $3.358 \mathrm{E}-6$ & $1.854 \mathrm{E}-2$ & $7.951 \mathrm{E}-7$ & $3.433 E-4$ & $1.350 \mathrm{E}-4$ & $7.527 \mathrm{E}-5$ & $1.247 \mathrm{E}-5$ & 2.349E-6 & $4.956 \mathrm{E}-8$ & $2.406 \mathrm{E}-6$ & $4.798 \mathrm{E}-7$ & $8.706 \mathrm{E}-9$ & $6.106 \mathrm{E}-7$ & $1.339 \mathrm{E}-8$ & $1.699 \mathrm{E}-7$ & $8.822 \mathrm{E}-4$ \\
\hline 28 & $2.112 \mathrm{E}-5$ & $3.546 \mathrm{E}-6$ & $1.851 \mathrm{E}-2$ & $9.564 \mathrm{E}-7$ & $3.277 \mathrm{E}-4$ & $1.371 \mathrm{E}-4$ & $7.838 \mathrm{E}-5$ & $1.419 \mathrm{E}-5$ & $2.567 \mathrm{E}-6$ & $5.512 \mathrm{E}-8$ & $2.888 \mathrm{E}-6$ & $5.577 \mathrm{E}-7$ & $1.082 \mathrm{E}-8$ & $7.916 \mathrm{E}-7$ & $1.314 \mathrm{E}-8$ & $1.679 \mathrm{E}-7$ & $9.500 \mathrm{E}-4$ \\
\hline 30 & $2.008 \mathrm{E}-5$ & $3.724 \mathrm{E}-6$ & $1.848 \mathrm{E}-2$ & $1.135 \mathrm{E}-6$ & $3.131 \mathrm{E}-4$ & $1.387 \mathrm{E}-4$ & $8.111 \mathrm{E}-5$ & $1.599 \mathrm{E}-5$ & 2.775E- 6 & $6.044 \mathrm{E}-8$ & $3.415 \mathrm{E}-6$ & $6.388 \mathrm{E}-7$ & $1.318 \mathrm{E}-8$ & $1.006 \mathrm{E}-6$ & $1.289 \mathrm{E}-8$ & $1.659 \mathrm{E}-7$ & $1.018 \mathrm{E}-3$ \\
\hline 32 & $1.906 \mathrm{E}-5$ & $3.890 \mathrm{E}-6$ & $1.845 \mathrm{E}-2$ & $1.330 \mathrm{E}-6$ & $2.996 \mathrm{E}-4$ & $1.398 \mathrm{E}-4$ & 8.345E-5 & $1.785 \mathrm{E}-5$ & $2.971 \mathrm{E}-6$ & $6.548 \mathrm{E}-8$ & 3.983E-6 & $7.223 \mathrm{E}-7$ & $1.579 \mathrm{E}-8$ & $1.257 \mathrm{E}-6$ & $1.264 \mathrm{E}-8$ & $1.638 \mathrm{E}-7$ & $1.085 \mathrm{E}-3$ \\
\hline 34 & $1.808 \mathrm{E}-5$ & $4.047 \mathrm{E}-6$ & $1.842 \mathrm{E}-2$ & $1.542 \mathrm{E}-6$ & $2.871 \mathrm{E}-4$ & $1.404 \mathrm{E}-4$ & $8.543 \mathrm{E}-5$ & $1.976 \mathrm{E}-5$ & $3.153 \mathrm{E}-6$ & $7.016 \mathrm{E}-8$ & $4.591 \mathrm{E}-6$ & $8.073 \mathrm{E}-7$ & $1.863 \mathrm{E}-8$ & $1.547 \mathrm{E}-6$ & $1.241 \mathrm{E}-8$ & $1.617 \mathrm{E}-7$ & $1.153 \mathrm{E}-3$ \\
\hline 36 & $1.713 \mathrm{E}-5$ & $4.193 \mathrm{E}-6$ & $1.838 \mathrm{E}-2$ & $1.770 \mathrm{E}-6$ & $2.755 \mathrm{E}-4$ & $1.407 \mathrm{E}-4$ & 8.705E-5 & $2.171 \mathrm{E}-5$ & $3.320 \mathrm{E}-6$ & 7.444E-8 & $5.235 \mathrm{E}-6$ & $8.929 \mathrm{E}-7$ & $2.167 \mathrm{E}-8$ & $1.878 \mathrm{E} \cdot 6$ & $1.218 \mathrm{E}-8$ & $1.596 \mathrm{E}-7$ & $1.221 \mathrm{E}-3$ \\
\hline 38 & $1.621 \mathrm{E}-5$ & $4.329 \mathrm{E}-6$ & $1.835 \mathrm{E}-2$ & $2.012 \mathrm{E}-6$ & $2.649 \mathrm{E}-4$ & $1.406 \mathrm{E}-4$ & 8.833E-5 & $2.370 \mathrm{E}-5$ & $3.470 \mathrm{E}-6$ & $7.831 \mathrm{E}-8$ & $5.912 \mathrm{E}-6$ & $9.782 \mathrm{E}-7$ & $2.490 \mathrm{E}-8$ & $2.250 \mathrm{E}-6$ & $1.197 \mathrm{E}-8$ & $1.575 \mathrm{E}-7$ & $1.288 \mathrm{E}-3$ \\
\hline 40 & $1.533 \mathrm{E}-5$ & $4.454 \mathrm{E}-6$ & $1.832 \mathrm{E}-2$ & $2.269 \mathrm{E}-6$ & $2.551 \mathrm{E}-4$ & $1.401 \mathrm{E}-4$ & $8.929 \mathrm{E}-5$ & $2.571 \mathrm{E}-5$ & 3.603E-6 & $8.173 E-8$ & $6.620 \mathrm{E}-6$ & $1.062 \mathrm{E}-6$ & $2.828 \mathrm{E}-8$ & $2.667 \mathrm{E}-6$ & $1.176 \mathrm{E}-8$ & $1.555 \mathrm{E}-7$ & $1.356 \mathrm{E}-3$ \\
\hline 42 & $1.447 \mathrm{E}-5$ & $4.570 \mathrm{E}-6$ & $1.828 \mathrm{E}-2$ & 2.538E-6 & $2.461 \mathrm{E}-4$ & $1.394 \mathrm{E}-4$ & 8.996E-5 & 2.774E-5 & 3.719E-6 & $8.469 \mathrm{E}-8$ & $7.354 \mathrm{E}-6$ & 1.145E-6 & $3.179 \mathrm{E}-8$ & $3.129 \mathrm{E}-6$ & $1.155 \mathrm{E}-8$ & $1.534 \mathrm{E}-7$ & $1.424 \mathrm{E}-3$ \\
\hline 44 & $1.365 \mathrm{E}-5$ & $4.675 \mathrm{E}-6$ & $1.825 \mathrm{E}-2$ & $2.818 \mathrm{E}-6$ & $2.379 \mathrm{E}-4$ & $1.384 \mathrm{E}-4$ & 9.036E-5 & $2.977 \mathrm{E}-5$ & $3.818 \mathrm{E}-6$ & $8.720 \mathrm{E}-8$ & $8.113 \mathrm{E}-6$ & $1.224 \mathrm{E}-6$ & $3.539 \mathrm{E}-8$ & $3.636 \mathrm{E}-6$ & $1.136 \mathrm{E}-8$ & $1.514 \mathrm{E}-7$ & $1.491 \mathrm{E}-3$ \\
\hline 46 & $1.286 \mathrm{E}-5$ & 4.772E-6 & $1.821 \mathrm{E}-2$ & $3.108 \mathrm{E}-6$ & $2.304 \mathrm{E}-4$ & $1.372 \mathrm{E}-4$ & $9.050 \mathrm{E}-5$ & $3.179 \mathrm{E}-5$ & $3.899 \mathrm{E}-6$ & 8.925E-8 & $8.891 \mathrm{E}-6$ & $1.300 \mathrm{E}-6$ & $3.904 \mathrm{E}-8$ & $4.190 \mathrm{E}-6$ & $1.118 \mathrm{E}-8$ & $1.494 \mathrm{E}-7$ & $1.559 \mathrm{E}-3$ \\
\hline 48 & $1.211 \mathrm{E}-5$ & $4.858 \mathrm{E}-6$ & $1.818 \mathrm{E}-2$ & $3.407 \mathrm{E}-6$ & $2.235 \mathrm{E}-4$ & $1.358 \mathrm{E}-4$ & $9.042 \mathrm{E}-5$ & $3.380 \mathrm{E}-5$ & $3.964 \mathrm{E}-6$ & $9.086 \mathrm{E}-8$ & $9.686 \mathrm{E}-6$ & $1.373 \mathrm{E}-6$ & $4.272 \mathrm{E}-8$ & $4.790 \mathrm{E}-6$ & $1.100 \mathrm{E}-8$ & $1.475 \mathrm{E}-7$ & $1.626 \mathrm{E}-3$ \\
\hline 50 & $1.139 \mathrm{E}-5$ & $4.936 \mathrm{E}-6$ & $1.814 \mathrm{E}-2$ & $3.712 \mathrm{E}-6$ & $2.172 \mathrm{E}-4$ & $1.342 \mathrm{E}-4$ & $9.014 \mathrm{E}-5$ & $3.580 \mathrm{E}-5$ & $4.012 \mathrm{E}-6$ & $9.206 \mathrm{E}-8$ & $1.049 \mathrm{E}-5$ & $1.440 \mathrm{E}-6$ & $4.639 \mathrm{E}-8$ & $5.437 \mathrm{E}-6$ & $1.083 \mathrm{E}-8$ & $1.456 \mathrm{E}-7$ & $1.694 \mathrm{E}-3$ \\
\hline 52 & $1.069 \mathrm{E}-5$ & $5.004 \mathrm{E}-6$ & $1.811 \mathrm{E}-2$ & $4.022 \mathrm{E}-6$ & $2.115 \mathrm{E}-4$ & $1.325 \mathrm{E}-4$ & $8.968 \mathrm{E}-5$ & $3.776 \mathrm{E}-5$ & $4.045 \mathrm{E}-6$ & $9.285 E-8$ & $1.131 \mathrm{E}-5$ & $1.503 \mathrm{E}-6$ & $5.001 \mathrm{E}-8$ & $6.130 \mathrm{E}-6$ & $1.067 \mathrm{E}-8$ & $1.438 \mathrm{E}-7$ & $1.761 \mathrm{E}-3$ \\
\hline 54 & $1.003 \mathrm{E}-5$ & $5.064 \mathrm{E}-6$ & $1.807 \mathrm{E}-2$ & $4.335 \mathrm{E}-6$ & $2.063 \mathrm{E}-4$ & $1.307 \mathrm{E}-4$ & $8.907 \mathrm{E}-5$ & $3.969 \mathrm{E}-5$ & $4.064 \mathrm{E}-6$ & $9.328 \mathrm{E}-8$ & $1.213 \mathrm{E}-5$ & $1.561 \mathrm{E}-6$ & $5.355 \mathrm{E}-8$ & $6.869 \mathrm{E}-6$ & $1.051 \mathrm{E}-8$ & $1.420 \mathrm{E}-7$ & $1.829 \mathrm{E}-3$ \\
\hline 56 & $9.405 E-6$ & $5.115 \mathrm{E}-6$ & $1.804 \mathrm{E}-2$ & 4.649E-6 & $2.016 \mathrm{E}-4$ & $1.288 \mathrm{E}-4$ & 8.832E-5 & $4.159 \mathrm{E}-5$ & 4.069E-6 & 9.335E-8 & $1.296 \mathrm{E}-5$ & $1.613 \mathrm{E}-6$ & $5.698 \mathrm{E}-8$ & $7.652 \mathrm{E}-6$ & $1.036 \mathrm{E}-8$ & $1.404 \mathrm{E}-7$ & $1.896 \mathrm{E}-3$ \\
\hline 58 & $8.807 \mathrm{E}-6$ & $5.159 \mathrm{E}-6$ & $1.800 \mathrm{E}-2$ & $4.963 \mathrm{E}-6$ & $1.973 \mathrm{E}-4$ & $1.269 \mathrm{E}-4$ & $8.746 \mathrm{E}-5$ & 4.343E-5 & $4.062 \mathrm{E}-6$ & $9.312 \mathrm{E}-8$ & $1.378 \mathrm{E}-5$ & $1.660 \mathrm{E}-6$ & $6.028 \mathrm{E}-8$ & $8.478 \mathrm{E}-6$ & $1.022 \mathrm{E}-8$ & $1.387 \mathrm{E}-7$ & $1.963 \mathrm{E}-3$ \\
\hline 60 & $8.240 \mathrm{E}-6$ & $5.194 \mathrm{E}-6$ & $1.796 \mathrm{E}-2$ & $5.276 \mathrm{E}-6$ & $1.933 \mathrm{E}-4$ & $1.249 \mathrm{E}-4$ & 8.651E-5 & $4.523 \mathrm{E}-5$ & $4.044 \mathrm{E}-6$ & $9.260 \mathrm{E}-8$ & $1.460 \mathrm{E}-5$ & $1.701 \mathrm{E}-6$ & $6.342 \mathrm{E}-8$ & 9.345E-6 & $1.009 \mathrm{E}-8$ & $1.372 \mathrm{E}-7$ & $2.031 \mathrm{E}-3$ \\
\hline
\end{tabular}


Table 4.9. Isotopic concentration vs burnup for Variant 10, State 1

\begin{tabular}{|c|c|c|c|c|c|c|c|c|c|c|c|c|c|c|c|c|c|}
\hline \multirow{2}{*}{$\begin{array}{c}\text { Burnup } \\
(\mathrm{MWd} / \mathrm{kg})\end{array}$} & \multicolumn{17}{|c|}{ Concentration (atoms $/ \mathrm{b} \cdot \mathrm{cm}$, in fuel) } \\
\hline & ${ }^{235} \mathrm{U}$ & ${ }^{236} \mathrm{U}$ & ${ }^{238} \mathrm{U}$ & ${ }^{238} \mathrm{Pu}$ & ${ }^{239} \mathrm{Pu}$ & ${ }^{240} \mathrm{Pu}$ & ${ }^{241} \mathrm{Pu}$ & ${ }^{242} \mathrm{Pu}$ & ${ }^{241} \mathrm{Am}$ & ${ }^{242 \mathrm{~m}} \mathrm{Am}$ & ${ }^{243} \mathrm{Am}$ & ${ }^{242} \mathrm{Cm}$ & ${ }^{243} \mathrm{Cm}$ & ${ }^{244} \mathrm{Cm}$ & ${ }^{135} \mathrm{Xe}$ & ${ }^{149} \mathrm{Sm}$ & Total FP \\
\hline 0 & $5.000 \mathrm{E}-5$ & 0.0 & $2.210 \mathrm{E}-2$ & $3.000 \mathrm{E}-5$ & $1.160 \mathrm{E}-3$ & $4.900 \mathrm{E}-4$ & $1.900 \mathrm{E}-4$ & $1.050 \mathrm{E}-4$ & $2.500 \mathrm{E}-5$ & 0.0 & 0.0 & 0.0 & 0.0 & 0.0 & $9.458 \mathrm{E}-9$ & $7.367 \mathrm{E}-8$ & $8.313 \mathrm{E}-8$ \\
\hline 2 & $4.872 \mathrm{E}-5$ & $3.507 \mathrm{E}-7$ & $2.207 \mathrm{E}-2$ & $2.963 \mathrm{E}-5$ & $1,133 \mathrm{E}-3$ & $4.899 \mathrm{E}-4$ & $1.970 \mathrm{E}-4$ & $1.050 \mathrm{E}-4$ & $2.508 \mathrm{E}-5$ & $1.745 \mathrm{E}-7$ & $2.453 \mathrm{E}-6$ & $8.667 \mathrm{E}-7$ & $2.461 \mathrm{E}-9$ & $7.296 \mathrm{E}-8$ & $2.546 \mathrm{E}-8$ & $3.799 \mathrm{E}-7$ & 8.334E-5 \\
\hline 4 & $4.747 \mathrm{E}-5$ & $6.913 \mathrm{E}-7$ & $2.204 \mathrm{E}-2$ & $2.946 \mathrm{E}-5$ & $1.108 \mathrm{E}-3$ & $4.894 \mathrm{E}-4$ & $2.035 \mathrm{E}-4$ & $1.052 \mathrm{E}-4$ & 2.521E-5 & $3.177 \mathrm{E}-7$ & $4.790 \mathrm{E}-6$ & $1.547 \mathrm{E}-6$ & 8.898E-9 & $2.842 \mathrm{E}-7$ & $2.541 \mathrm{E}-8$ & $4.944 \mathrm{E}-7$ & $1.666 \mathrm{E}-4$ \\
\hline 6 & $4.624 \mathrm{E}-5$ & $1.021 \mathrm{E}-6$ & $2.200 \mathrm{E}-2$ & $2.944 \mathrm{E}-5$ & $1.083 \mathrm{E}-3$ & $4.887 \mathrm{E}-4$ & $2.096 \mathrm{E}-4$ & $1.054 \mathrm{E}-4$ & $2.536 \mathrm{E}-5$ & $4.351 \mathrm{E}-7$ & $7.009 \mathrm{E}-6$ & $2.081 \mathrm{E}-6$ & $1.809 \mathrm{E}-8$ & $6.210 \mathrm{E}-7$ & $2.534 \mathrm{E}-8$ & $5.370 \mathrm{E}-7$ & $2.500 \mathrm{E}-4$ \\
\hline 8 & $4.503 \mathrm{E}-5$ & $1.340 \mathrm{E}-6$ & $2.197 \mathrm{E}-2$ & $2.955 \mathrm{E}-5$ & $1.060 \mathrm{E}-3$ & $4.879 \mathrm{E}-4$ & $2.153 \mathrm{E}-4$ & $1.057 \mathrm{E}-4$ & $2.555 \mathrm{E}-5$ & $5.314 \mathrm{E}-7$ & $9.117 \mathrm{E}-6$ & $2.505 \mathrm{E}-6$ & $2.913 \mathrm{E}-8$ & $1.072 \mathrm{E}-6$ & $2.526 \mathrm{E}-8$ & $5.556 \mathrm{E}-7$ & $3.335 \mathrm{E}-4$ \\
\hline 10 & $4.384 \mathrm{E}-5$ & $1.648 \mathrm{E}-6$ & $2.194 \mathrm{E}-2$ & $2.975 \mathrm{E}-5$ & $1.037 \mathrm{E}-3$ & $4.868 \mathrm{E}-4$ & $2.205 E-4$ & $1.061 E-4$ & $2.576 \mathrm{E}-5$ & $6.105 \mathrm{E}-7$ & $1.112 \mathrm{E}-5$ & $2.844 \mathrm{E}-6$ & $4.133 \mathrm{E}-8$ & $1.625 \mathrm{E}-6$ & $2.518 \mathrm{E}-8$ & $5.655 \mathrm{E}-7$ & $4.170 \mathrm{E}-4$ \\
\hline 12 & $4.268 \mathrm{E}-5$ & $1.946 \mathrm{E}-6$ & $2.190 \mathrm{E}-2$ & $3.003 \mathrm{E}-5$ & $1.015 \mathrm{E}-3$ & $4.855 \mathrm{E}-4$ & $2.253 \mathrm{E}-4$ & $1.065 \mathrm{E}-4$ & $2.598 \mathrm{E}-5$ & $6.756 \mathrm{E}-7$ & $1.303 \mathrm{E}-5$ & $3.118 \mathrm{E}-6$ & $5.418 \mathrm{E}-8$ & $2.272 \mathrm{E}-6$ & $2.510 \mathrm{E}-8$ & $5.717 \mathrm{E}-7$ & $5.005 \mathrm{E}-4$ \\
\hline 14 & $4.154 \mathrm{E}-5$ & $2.233 \mathrm{E}-6$ & $2.187 \mathrm{E}-2$ & $3.037 \mathrm{E}-5$ & $9.934 \mathrm{E}-4$ & $4.841 \mathrm{E}-4$ & $2.297 \mathrm{E}-4$ & $1.070 \mathrm{E}-4$ & $2.623 \mathrm{E}-5$ & $7.295 \mathrm{E}-7$ & $1.485 \mathrm{E}-5$ & $3.343 \mathrm{E}-6$ & $6.733 \mathrm{E}-8$ & $3.004 \mathrm{E}-6$ & $2.501 \mathrm{E}-8$ & $5.759 \mathrm{E}-7$ & $5.839 \mathrm{E}-4$ \\
\hline 16 & $4.042 \mathrm{E}-5$ & $2.511 \mathrm{E}-6$ & $2.183 \mathrm{E}-2$ & $3.075 \mathrm{E}-5$ & $9.725 \mathrm{E}-4$ & $4.825 \mathrm{E}-4$ & $2.337 \mathrm{E}-4$ & $1.077 \mathrm{E}-4$ & $2.648 \mathrm{E}-5$ & $7.743 \mathrm{E}-7$ & $1.659 \mathrm{E}-5$ & $3.531 \mathrm{E}-6$ & $8.051 \mathrm{E}-8$ & $3.813 \mathrm{E}-6$ & $2.492 \mathrm{E}-8$ & $5.788 \mathrm{E}-7$ & $6.673 \mathrm{E}-4$ \\
\hline 18 & $3.933 \mathrm{E}-5$ & $2.778 \mathrm{E}-6$ & $2.180 \mathrm{E}-2$ & $3.117 \mathrm{E}-5$ & $9.522 \mathrm{E}-4$ & $4.807 \mathrm{E}-4$ & $2.374 \mathrm{E}-4$ & $1.083 \mathrm{E}-4$ & $2.674 \mathrm{E}-5$ & $8.116 \mathrm{E}-7$ & $1.826 \mathrm{E}-5$ & $3.691 \mathrm{E}-6$ & $9.354 \mathrm{E}-8$ & $4.693 \mathrm{E}-6$ & $2.483 \mathrm{E}-8$ & 5.807E-7 & $7.507 \mathrm{E}-4$ \\
\hline 20 & $3.825 \mathrm{E}-5$ & $3.037 \mathrm{E}-6$ & $2.177 \mathrm{E}-2$ & $3.163 \mathrm{E}-5$ & $9.324 \mathrm{E}-4$ & $4.787 \mathrm{E}-4$ & $2.407 \mathrm{E}-4$ & $1.090 \mathrm{E}-4$ & $2.700 \mathrm{E}-5$ & $8.428 \mathrm{E}-7$ & $1.985 \mathrm{E}-5$ & $3.830 \mathrm{E}-6$ & $1.063 \mathrm{E}-7$ & $5.636 \mathrm{E}-6$ & $2.473 \mathrm{E}-8$ & $5.817 \mathrm{E}-7$ & $8.341 E-4$ \\
\hline 22 & $3.720 \mathrm{E}-5$ & $3.285 \mathrm{E}-6$ & $2.173 \mathrm{E}-2$ & $3.211 \mathrm{E}-5$ & $9.133 \mathrm{E}-4$ & $4.766 \mathrm{E}-4$ & $2.438 \mathrm{E}-4$ & $1.098 \mathrm{E}-4$ & $2.726 \mathrm{E}-5$ & $8.690 \mathrm{E}-7$ & $2.138 \mathrm{E}-5$ & $3.954 \mathrm{E}-6$ & $1.187 \mathrm{E}-7$ & $6.637 \mathrm{E}-6$ & $2.464 \mathrm{E}-8$ & $5.819 \mathrm{E}-7$ & $9.174 \mathrm{E}-4$ \\
\hline 24 & $3.617 \mathrm{E}-5$ & $3.525 \mathrm{E}-6$ & $2.170 \mathrm{E}-2$ & $3.262 \mathrm{E}-5$ & $8.946 \mathrm{E}-4$ & $4.743 \mathrm{E}-4$ & $2.465 \mathrm{E}-4$ & $1.106 \mathrm{E}-4$ & $2.751 \mathrm{E}-5$ & $8.913 \mathrm{E}-7$ & $2.285 \mathrm{E}-5$ & $4.064 \mathrm{E}-6$ & $1.307 \mathrm{E}-7$ & $7.691 \mathrm{E}-6$ & $2.454 \mathrm{E}-8$ & $5.816 \mathrm{E}-7$ & $1.001 \mathrm{E}-3$ \\
\hline 26 & $3.515 \mathrm{E}-5$ & $3.756 \mathrm{E}-6$ & $2.166 \mathrm{E}-2$ & $3.314 \mathrm{E}-5$ & $8.766 \mathrm{E}-4$ & $4.719 \mathrm{E}-4$ & $2,489 \mathrm{E}-4$ & $1.115 \mathrm{E}-4$ & $2.776 \mathrm{E}-5$ & $9.101 \mathrm{E}-7$ & $2.426 \mathrm{E}-5$ & $4.166 \mathrm{E}-6$ & $1.423 \mathrm{E}-7$ & $8.793 \mathrm{E}-6$ & $2.444 \mathrm{E}-8$ & $5.808 \mathrm{E}-7$ & $1.084 \mathrm{E}-3$ \\
\hline 28 & $3.416 \mathrm{E}-5$ & 3.977E-6 & $2.163 \mathrm{E}-2$ & $3.368 \mathrm{E}-5$ & $8.590 \mathrm{E}-4$ & $4.694 \mathrm{E}-4$ & $2.510 \mathrm{E}-4$ & $1.124 \mathrm{E}-4$ & $2.800 \mathrm{E}-5$ & $9.262 \mathrm{E}-7$ & $2.562 \mathrm{E}-5$ & $4.261 \mathrm{E}-6$ & $1.534 \mathrm{E}-7$ & $9.939 \mathrm{E}-6$ & $2.435 \mathrm{E}-8$ & $5.795 \mathrm{E}-7$ & $1.167 \mathrm{E}-3$ \\
\hline 30 & $3.319 \mathrm{E}-5$ & $4.190 \mathrm{E}-6$ & $2.159 \mathrm{E}-2$ & $3.424 \mathrm{E}-5$ & $8.420 \mathrm{E}-4$ & $4.667 \mathrm{E}-4$ & $2.529 \mathrm{E}-4$ & $1.134 \mathrm{E}-4$ & $2.823 \mathrm{E}-5$ & $9.400 \mathrm{E}-7$ & $2.693 \mathrm{E}-5$ & $4.350 \mathrm{E}-6$ & $1.641 \mathrm{E}-7$ & $1.112 \mathrm{E}-5$ & $2.425 \mathrm{E}-8$ & 5.779E-7 & $1.250 \mathrm{E}-3$ \\
\hline 32 & $3.224 \mathrm{E}-5$ & $4.394 \mathrm{E}-6$ & $2.156 \mathrm{E}-2$ & $3.481 \mathrm{E}-5$ & $8.254 \mathrm{E}-4$ & $4.638 \mathrm{E}-4$ & $2.545 \mathrm{E}-4$ & $1.144 \mathrm{E}-4$ & $2.845 \mathrm{E}-5$ & $9.518 \mathrm{E}-7$ & $2.819 \mathrm{E}-5$ & $4.435 \mathrm{E}-6$ & $1.744 \mathrm{E}-7$ & $1.235 \mathrm{E}-5$ & $2.415 \mathrm{E}-8$ & $5.759 \mathrm{E}-7$ & $1.334 \mathrm{E}-3$ \\
\hline 34 & $3.131 \mathrm{E}-5$ & $4.590 \mathrm{E}-6$ & $2.152 \mathrm{E}-2$ & $3.539 \mathrm{E}-5$ & $8.094 \mathrm{E}-4$ & $4.609 \mathrm{E}-4$ & $2.559 \mathrm{E}-4$ & $1.154 \mathrm{E}-4$ & $2.866 \mathrm{E}-5$ & $9.619 \mathrm{E}-7$ & $2.941 \mathrm{E}-5$ & $4.516 \mathrm{E}-6$ & $1.843 \mathrm{E}-7$ & $1.360 \mathrm{E}-5$ & $2.405 \mathrm{E}-8$ & $5.736 \mathrm{E}-7$ & $1.417 \mathrm{E}-3$ \\
\hline 36 & $3.039 \mathrm{E}-5$ & $4.778 \mathrm{E}-6$ & $2.149 \mathrm{E}-2$ & $3.598 \mathrm{E}-5$ & $7.938 \mathrm{E}-4$ & $4.578 \mathrm{E}-4$ & $2.571 \mathrm{E}-4$ & $1.165 \mathrm{E}-4$ & $2.884 \mathrm{E}-5$ & $9.705 \mathrm{E}-7$ & $3.058 \mathrm{E}-5$ & $4.593 \mathrm{E}-6$ & $1.937 \mathrm{E}-7$ & $1.489 \mathrm{E}-5$ & $2.395 \mathrm{E}-8$ & $5.710 \mathrm{E}-7$ & $1.500 \mathrm{E}-3$ \\
\hline 38 & $2.950 \mathrm{E}-5$ & $4.958 \mathrm{E}-6$ & $2.145 \mathrm{E}-2$ & $3.658 \mathrm{E}-5$ & $7.786 \mathrm{E}-4$ & $4.546 \mathrm{E}-4$ & $2.580 \mathrm{E}-4$ & $1.175 \mathrm{E}-4$ & $2.902 \mathrm{E}-5$ & $9.778 \mathrm{E}-7$ & $3.172 \mathrm{E}-5$ & $4.668 \mathrm{E}-6$ & $2.028 \mathrm{E}-7$ & $1.620 \mathrm{E}-5$ & $2.384 \mathrm{E}-8$ & $5.683 \mathrm{E}-7$ & $1.583 \mathrm{E}-3$ \\
\hline 40 & $2.862 \mathrm{E}-5$ & $5.129 \mathrm{E}-6$ & $2.141 \mathrm{E}-2$ & $3.719 \mathrm{E}-5$ & $7.640 \mathrm{E}-4$ & $4.513 \mathrm{E}-4$ & $2.588 \mathrm{E}-4$ & $1.186 \mathrm{E}-4$ & $2.917 \mathrm{E}-5$ & $9.839 \mathrm{E}-7$ & $3.283 \mathrm{E}-5$ & $4.741 \mathrm{E}-6$ & $2.115 \mathrm{E}-7$ & $1.753 \mathrm{E}-5$ & $2.374 \mathrm{E}-8$ & $5.653 \mathrm{E}-7$ & $1.666 \mathrm{E}-3$ \\
\hline 42 & 2.777 E-5 & $5.293 \mathrm{E}-6$ & $2.138 \mathrm{E}-2$ & $3.780 \mathrm{E}-5$ & $7.498 \mathrm{E}-4$ & $4.479 \mathrm{E}-4$ & $2.593 \mathrm{E}-4$ & $1.197 \mathrm{E}-4$ & $2.931 \mathrm{E}-5$ & $9.889 \mathrm{E}-7$ & 3.389E-5 & $4.810 \mathrm{E}-6$ & $2.198 \mathrm{E}-7$ & $1.889 \mathrm{E}-5$ & $2.364 \mathrm{E}-8$ & $5.622 \mathrm{E}-7$ & $1.749 \mathrm{E}-3$ \\
\hline 44 & $2.693 \mathrm{E}-5$ & $5.450 \mathrm{E}-6$ & $2.134 \mathrm{E}-2$ & $3.842 \mathrm{E}-5$ & $7.360 \mathrm{E}-4$ & $4.444 \mathrm{E}-4$ & $2.597 \mathrm{E}-4$ & $1.209 \mathrm{E}-4$ & $2.943 \mathrm{E}-5$ & $9.930 \mathrm{E}-7$ & $3.493 \mathrm{E}-5$ & $4.878 \mathrm{E}-6$ & $2.278 \mathrm{E}-7$ & $2.026 \mathrm{E}-5$ & $2.354 \mathrm{E}-8$ & $5.591 \mathrm{E}-7$ & $1.832 \mathrm{E}-3$ \\
\hline 46 & $2.612 \mathrm{E}-5$ & $5.598 \mathrm{E}-6$ & $2.130 \mathrm{E}-2$ & $3.904 \mathrm{E}-5$ & $7.226 \mathrm{E}-4$ & $4.408 \mathrm{E}-4$ & $2.598 \mathrm{E}-4$ & $1.220 \mathrm{E}-4$ & $2.953 \mathrm{E}-5$ & $9.961 \mathrm{E}-7$ & $3.594 \mathrm{E}-5$ & $4.942 \mathrm{E}-6$ & $2.355 \mathrm{E}-7$ & $2.165 \mathrm{E}-5$ & $2.344 \mathrm{E}-8$ & $5.558 \mathrm{E}-7$ & $1.915 \mathrm{E}-3$ \\
\hline 48 & $2.532 \mathrm{E}-5$ & $5.740 \mathrm{E}-6$ & $2.127 \mathrm{E}-2$ & $3.967 \mathrm{E}-5$ & $7.096 \mathrm{E}-4$ & $4.371 \mathrm{E}-4$ & $2.598 \mathrm{E}-4$ & $1.232 \mathrm{E}-4$ & $2.961 \mathrm{E}-5$ & $9.983 \mathrm{E}-7$ & $3.691 \mathrm{E}-5$ & $5.005 \mathrm{E}-6$ & $2.428 \mathrm{E}-7$ & $2.306 \mathrm{E}-5$ & $2.333 \mathrm{E}-8$ & $5.525 \mathrm{E}-7$ & $1.998 \mathrm{E}-3$ \\
\hline 50 & $2.453 \mathrm{E}-5$ & $5.874 \mathrm{E}-6$ & $2.123 \mathrm{E}-2$ & $4.030 \mathrm{E}-5$ & $6.971 \mathrm{E}-4$ & $4.333 \mathrm{E}-4$ & $2.597 \mathrm{E}-4$ & $1.243 \mathrm{E}-4$ & 2.967E-5 & $9.997 \mathrm{E}-7$ & $3.786 \mathrm{E}-5$ & $5.065 \mathrm{E}-6$ & $2.499 \mathrm{E}-7$ & $2.448 \mathrm{E}-5$ & $2.323 \mathrm{E}-8$ & $5.490 \mathrm{E}-7$ & $2.081 \mathrm{E}-3$ \\
\hline 52 & $2.377 \mathrm{E}-5$ & $6.001 \mathrm{E}-6$ & $2.119 \mathrm{E}-2$ & $4.094 \mathrm{E}-5$ & $6.849 \mathrm{E}-4$ & $4.294 \mathrm{E}-4$ & $2.594 \mathrm{E}-4$ & $1.255 \mathrm{E}-4$ & $2.971 \mathrm{E}-5$ & $1.000 \mathrm{E}-6$ & $3.878 \mathrm{E}-5$ & $5.122 \mathrm{E}-6$ & $2.567 \mathrm{E}-7$ & $2.592 \mathrm{E}-5$ & $2.313 \mathrm{E}-8$ & $5.455 \mathrm{E}-7$ & $2.164 \mathrm{E}-3$ \\
\hline 54 & $2.302 \mathrm{E}-5$ & $6.121 \mathrm{E}-6$ & $2.116 \mathrm{E}-2$ & $4.157 \mathrm{E}-5$ & $6.731 \mathrm{E}-4$ & $4.255 \mathrm{E}-4$ & $2.589 \mathrm{E}-4$ & $1.267 \mathrm{E}-4$ & 2.973E-5 & $1.000 \mathrm{E}-6$ & $3.967 \mathrm{E}-5$ & $5.176 \mathrm{E}-6$ & $2.631 \mathrm{E}-7$ & $2.736 \mathrm{E}-5$ & $2.302 \mathrm{E}-8$ & $5.419 \mathrm{E}-7$ & $2.246 \mathrm{E}-3$ \\
\hline 56 & $2.230 \mathrm{E}-5$ & $6.235 \mathrm{E}-6$ & $2.112 \mathrm{E}-2$ & $4.220 \mathrm{E}-5$ & $6.617 \mathrm{E}-4$ & $4.215 \mathrm{E}-4$ & $2.584 \mathrm{E}-4$ & $1.278 \mathrm{E}-4$ & $2.973 \mathrm{E}-5$ & $9.992 \mathrm{E}-7$ & $4.054 \mathrm{E}-5$ & $5.228 \mathrm{E}-6$ & $2.693 \mathrm{E}-7$ & $2.881 \mathrm{E}-5$ & $2.292 \mathrm{E}-8$ & $5.384 \mathrm{E}-7$ & $2.329 \mathrm{E}-3$ \\
\hline 58 & $2.158 \mathrm{E}-5$ & $6.342 \mathrm{E}-6$ & $2.108 \mathrm{E}-2$ & $4.283 \mathrm{E}-5$ & $6.506 \mathrm{E}-4$ & $4.174 \mathrm{E}-4$ & $2.577 \mathrm{E}-4$ & $1.290 \mathrm{E}-4$ & $2.971 \mathrm{E}-5$ & $9.976 \mathrm{E}-7$ & $4.139 \mathrm{E}-5$ & $5.276 \mathrm{E}-6$ & 2.753E-7 & $3.027 \mathrm{E}-5$ & $2.282 \mathrm{E}-8$ & $5.348 \mathrm{E}-7$ & $2.412 \mathrm{E}-3$ \\
\hline 60 & $2.089 \mathrm{E}-5$ & $6.442 \mathrm{E}-6$ & 2.105E-2 & $4.346 \mathrm{E}-5$ & $6.399 \mathrm{E}-4$ & $4.133 E-4$ & $2.568 \mathrm{E}-4$ & $1.302 \mathrm{E}-4$ & $2.967 \mathrm{E}-5$ & $9.952 \mathrm{E}-7$ & 4.221E-5 & $5.322 \mathrm{E}-6$ & $2.809 \mathrm{E}-7$ & $3.174 \mathrm{E}-5$ & $2.272 \mathrm{E}-8$ & $5.312 \mathrm{E}-7$ & $2.495 \mathrm{E}-3$ \\
\hline
\end{tabular}


Table 4.10. Isotopic concentration in pin 1 vs burnup for Variant 11, State 1

\begin{tabular}{|c|c|c|c|c|c|c|c|c|c|c|c|}
\hline \multirow{2}{*}{$\begin{array}{c}\text { Burnup } \\
\text { (MWd/kg) }\end{array}$} & \multicolumn{11}{|c|}{ Concentration (atoms $/ \mathrm{b} \cdot \mathrm{cm}$, in fuel) } \\
\hline & ${ }^{235} \mathrm{U}$ & ${ }^{236} \mathrm{U}$ & ${ }^{238} \mathrm{U}$ & ${ }^{238} \mathrm{Pu}$ & ${ }^{239} \mathrm{Pu}$ & ${ }^{240} \mathrm{Pu}$ & ${ }^{241} \mathrm{Pu}$ & ${ }^{242} \mathrm{Pu}$ & ${ }^{135} \mathrm{Xe}$ & ${ }^{149} \mathrm{Sm}$ & Total FP \\
\hline 0 & $8.737 \mathrm{E}-4$ & 0.0 & $1.874 \mathrm{E}-2$ & 0.0 & 0.0 & 0.0 & 0.0 & 0.0 & $9.458 \mathrm{E}-9$ & $7.367 \mathrm{E}-8$ & $8.312 \mathrm{E}-8$ \\
\hline 2 & 8.241E-4 & $9.130 \mathrm{E}-6$ & $1.872 \mathrm{E}-2$ & $1.364 \mathrm{E}-9$ & $1.699 \mathrm{E}-5$ & $5.439 \mathrm{E}-7$ & $4.536 \mathrm{E}-8$ & 4.835E-10 & $9.716 \mathrm{E}-9$ & $7.450 \mathrm{E}-8$ & $7.048 \mathrm{E}-5$ \\
\hline 4 & $7.772 \mathrm{E}-4$ & $1.770 \mathrm{E}-5$ & $1.870 \mathrm{E}-2$ & $8.656 \mathrm{E}-9$ & $3.243 \mathrm{E}-5$ & $2.000 \mathrm{E}-6$ & $3.378 \mathrm{E}-7$ & $7.546 \mathrm{E}-9$ & $9.767 \mathrm{E}-9$ & $7.895 \mathrm{E}-8$ & $1.408 \mathrm{E}-4$ \\
\hline 6 & $7.327 \mathrm{E}-4$ & 2.579E-5 & $1.868 \mathrm{E}-2$ & 2.499E-8 & 4.552E-5 & 4.003E-6 & $9.889 \mathrm{E}-7$ & $3.442 \mathrm{E}-8$ & $9.771 \mathrm{E}-9$ & $8.272 \mathrm{E}-8$ & $2.115 \mathrm{E}-4$ \\
\hline 8 & $6.904 \mathrm{E}-4$ & 3.341E-5 & $1.865 \mathrm{E}-2$ & $5.303 \mathrm{E}-8$ & $5.665 \mathrm{E}-5$ & $6.334 \mathrm{E}-6$ & $2.002 \mathrm{E}-6$ & $9.622 \mathrm{E}-8$ & $9.739 \mathrm{E}-9$ & $8.584 \mathrm{E}-8$ & $2.822 \mathrm{E}-4$ \\
\hline 10 & $6.500 \mathrm{E}-4$ & $4.061 \mathrm{E}-5$ & $1.863 \mathrm{E}-2$ & $9.520 \mathrm{E}-8$ & $6.615 \mathrm{E}-5$ & $8.860 \mathrm{E}-6$ & $3.332 \mathrm{E}-6$ & $2.068 \mathrm{E}-7$ & $9.681 \mathrm{E}-9$ & $8.840 \mathrm{E}-8$ & $3.529 \mathrm{E}-4$ \\
\hline 12 & $6.115 \mathrm{E}-4$ & $4.741 \mathrm{E}-5$ & $1.860 \mathrm{E}-2$ & $1.538 \mathrm{E}-7$ & $7.427 \mathrm{E}-5$ & $1.150 \mathrm{E}-5$ & $4.915 \mathrm{E}-6$ & $3.776 \mathrm{E}-7$ & $9.603 \mathrm{E}-9$ & $9.047 \mathrm{E}-8$ & $4.236 \mathrm{E}-4$ \\
\hline 14 & $5.748 \mathrm{E}-4$ & $5.383 \mathrm{E}-5$ & $1.858 \mathrm{E}-2$ & 2.307E-7 & $8.121 \mathrm{E}-5$ & $1.419 \mathrm{E}-5$ & $6.687 \mathrm{E}-6$ & $6.172 \mathrm{E}-7$ & $9.509 \mathrm{E}-9$ & $9.211 \mathrm{E}-8$ & $4.942 \mathrm{E}-4$ \\
\hline 16 & $5.397 \mathrm{E}-4$ & $5.988 \mathrm{E}-5$ & $1.855 \mathrm{E}-2$ & $3.281 \mathrm{E}-7$ & $8.714 \mathrm{E}-5$ & $1.690 \mathrm{E}-5$ & $8.590 \mathrm{E}-6$ & 9.317E-7 & $9.403 \mathrm{E}-9$ & $9.338 \mathrm{E}-8$ & $5.647 \mathrm{E}-4$ \\
\hline 18 & $5.062 \mathrm{E}-4$ & $6.557 \mathrm{E}-5$ & $1.853 \mathrm{E}-2$ & $4.475 \mathrm{E}-7$ & $9.220 \mathrm{E}-5$ & $1.959 \mathrm{E}-5$ & 1.057E-5 & $1.325 \mathrm{E}-6$ & $9.286 \mathrm{E}-9$ & $9.430 \mathrm{E}-8$ & OE-4 \\
\hline 20 & 4.742E-4 & $7.093 \mathrm{E}-5$ & $1.850 \mathrm{E}-2$ & $5.906 \mathrm{E}-7$ & $9.649 \mathrm{E}-5$ & $2.225 \mathrm{E}-5$ & $1.259 \mathrm{E}-5$ & $1.799 \mathrm{E}-6$ & $9.161 E-9$ & $9.492 \mathrm{E}-8$ & $3 E-4$ \\
\hline 22 & $4.437 \mathrm{E}-4$ & $7.595 \mathrm{E}-5$ & $1.847 \mathrm{E}-2$ & $7.585 \mathrm{E}-7$ & $1.001 \mathrm{E}-4$ & $2.485 \mathrm{E}-5$ & 1.46 & $2.354 \mathrm{E}-6$ & $9.029 \mathrm{E}-9$ & 9.52 & $5 \mathrm{E}-4$ \\
\hline 24 & $4.146 \mathrm{E}-4$ & 8.065E-5 & $1.844 \mathrm{E}-2$ & $9.526 \mathrm{E}-7$ & $1.032 \mathrm{E}-4$ & $2.740 \mathrm{E}-5$ & 1.6 & $2.989 \mathrm{E}-6$ & 8.892E-9 & $E-8$ & $6 \mathrm{E}-4$ \\
\hline 26 & $3.869 \mathrm{E}-4$ & $8.504 \mathrm{E}-5$ & $1.842 \mathrm{E}-2$ & $1.174 \mathrm{E}-6$ & $1.057 \mathrm{E}-4$ & $2.986 \mathrm{E}-5$ & $1.855 \mathrm{E}-5$ & $3.702 \mathrm{E}-6$ & 8.750E-9 & $E-8$ & 5E-4 \\
\hline 28 & $3.605 \mathrm{E}-4$ & $8.912 \mathrm{E}-5$ & $1.839 \mathrm{E}-2$ & $1.422 \mathrm{E}-6$ & $1.078 \mathrm{E}-4$ & $3.225 \mathrm{E}-5$ & $2.042 \mathrm{E}-5$ & 4.491E-6 & $8.606 \mathrm{E}-9$ & 9.505E-8 & 3E-4 \\
\hline 30 & 3.354E-4 & $9.291 \mathrm{E}-5$ & $1.836 \mathrm{E}-2$ & 1.699E-6 & $1.096 \mathrm{E}-4$ & $3.454 \mathrm{E}-5$ & 2.2 & $5.351 \mathrm{E}-6$ & 8.459E-9 & $9.462 \mathrm{E}-8$ & $5 E-3$ \\
\hline 32 & $3.116 \mathrm{E}-4$ & 9.641E-5 & $1.833 \mathrm{E}-2$ & $2.003 \mathrm{E}-6$ & $1.109 \mathrm{E}-4$ & $3.674 \mathrm{E}-5$ & 2.39 & $6.279 \mathrm{E}-6$ & $8.311 \mathrm{E}-9$ & $9.406 \mathrm{E}-8$ & $5 E-3$ \\
\hline 34 & $2.890 \mathrm{E}-4$ & 9.964E-5 & $1.830 \mathrm{E}-2$ & $2.336 \mathrm{E}-6$ & $1.121 \mathrm{E}-4$ & $4 \mathrm{E}-5$ & 2.5 & $7.271 \mathrm{E}-6$ & $8.164 \mathrm{E}-9$ & $9.339 \mathrm{E}-8$ & $1.194 \mathrm{E}-3$ \\
\hline 36 & $2.676 \mathrm{E}-4$ & $1.026 \mathrm{E}-4$ & $1.827 \mathrm{E}-2$ & $2.696 \mathrm{E}-6$ & $1.129 \mathrm{E}-4$ & 4.0 & -5 & $8.322 \mathrm{E}-6$ & $8.016 \mathrm{E}-9$ & $9.262 \mathrm{E}-8$ & $1.263 \mathrm{E}-3$ \\
\hline 38 & $2.474 \mathrm{E}-4$ & $1.053 \mathrm{E}-4$ & $1.824 \mathrm{E}-2$ & $3.083 \mathrm{E}-6$ & $1.136 \mathrm{E}-4$ & $4.274 \mathrm{E}-5$ & 2.839 & $9.427 \mathrm{E}-6$ & 7.870 E-9 & -8 & $3 \mathrm{E}-3$ \\
\hline 40 & $2.283 \mathrm{E}-4$ & $1.077 \mathrm{E}-4$ & $1.821 \mathrm{E}-2$ & $3.496 \mathrm{E}-6$ & $1.140 \mathrm{E}-4$ & $4.453 \mathrm{E}-5$ & $2.967 \mathrm{E}-5$ & $1.058 \mathrm{E}-5$ & $7.726 \mathrm{E}-9$ & 9.08 & $2 \mathrm{E}-3$ \\
\hline 42 & $2.103 \mathrm{E}-4$ & $1.099 \mathrm{E}-4$ & $1.818 \mathrm{E}-2$ & $3.934 \mathrm{E}-6$ & $1.144 \mathrm{E}-4$ & $4.622 \mathrm{E}-5$ & $3.084 \mathrm{E}-5$ & $1.178 \mathrm{E}-5$ & $7.585 \mathrm{E}-9$ & E-8 & $1.471 \mathrm{E}-3$ \\
\hline 44 & $1.933 \mathrm{E}-4$ & $1.118 \mathrm{E}-4$ & $1.814 \mathrm{E}-2$ & 4.394E-6 & $1.145 \mathrm{E}-4$ & 4.781E-5 & $3.192 \mathrm{E}-5$ & $1.302 \mathrm{E}-5$ & $7.446 \mathrm{E}-9$ & 8.891E-8 & $1.540 \mathrm{E}-3$ \\
\hline 46 & $1.774 \mathrm{E}-4$ & 1.135E-4 & $1.811 \mathrm{E}-2$ & $4.876 \mathrm{E}-6$ & $1.146 \mathrm{E}-4$ & $4.930 \mathrm{E}-5$ & $3.289 \mathrm{E}-5$ & $1.429 \mathrm{E}-5$ & 7.312E-9 & $8.790 \mathrm{E}-8$ & $1.609 \mathrm{E}-3$ \\
\hline 48 & $1.625 \mathrm{E}-4$ & $1.150 \mathrm{E}-4$ & $1.808 \mathrm{E}-2$ & 5.377E-6 & $1.146 \mathrm{E}-4$ & $5.069 \mathrm{E}-5$ & 3.377E-5 & $1.559 \mathrm{E}-5$ & $7.182 \mathrm{E}-9$ & $8.689 \mathrm{E}-8$ & $1.678 \mathrm{E}-3$ \\
\hline 50 & $1.486 \mathrm{E}-4$ & $1.163 \mathrm{E}-4$ & 1.804E-2 & $5.896 \mathrm{E}-6$ & $1.145 \mathrm{E}-4$ & $5.198 \mathrm{E}-5$ & $3.456 \mathrm{E}-5$ & $1.691 \mathrm{E}-5$ & 7.057E-9 & 8.587E-8 & $1.746 \mathrm{E}-3$ \\
\hline 52 & $1.356 \mathrm{E}-4$ & $1.173 \mathrm{E}-4$ & $1.801 \mathrm{E}-2$ & $6.429 \mathrm{E}-6$ & 1.143E-4 & 5.317E-5 & $3.527 \mathrm{E}-5$ & $1.825 \mathrm{E}-5$ & $6.936 \mathrm{E}-9$ & $8.486 \mathrm{E}-8$ & $1.815 \mathrm{E}-3$ \\
\hline 54 & $1.236 \mathrm{E}-4$ & $1.182 \mathrm{E}-4$ & $1.797 \mathrm{E}-2$ & $6.974 \mathrm{E}-6$ & $1.141 \mathrm{E}-4$ & $5.428 \mathrm{E}-5$ & $3.590 \mathrm{E}-5$ & $1.961 \mathrm{E}-5$ & $6.821 \mathrm{E}-9$ & $8.388 \mathrm{E}-8$ & $1.884 \mathrm{E}-3$ \\
\hline 56 & $1.124 \mathrm{E}-4$ & $1.188 \mathrm{E}-4$ & $1.794 \mathrm{E}-2$ & 7.530E-6 & 1.139E-4 & $5.530 \mathrm{E}-5$ & $3.645 E-5$ & $2.098 \mathrm{E}-5$ & $6.712 \mathrm{E}-9$ & 8.292E-8 & $1.952 \mathrm{E}-3$ \\
\hline 58 & $1.020 \mathrm{E}-4$ & 1.193E-4 & $1.790 \mathrm{E}-2$ & 8.093E-6 & $1.136 \mathrm{E}-4$ & 5.624E-5 & $3.694 \mathrm{E}-5$ & $2.235 \mathrm{E}-5$ & 6.609E-9 & $8.200 \mathrm{E}-8$ & $2.021 \mathrm{E}-3$ \\
\hline 60 & $9.241 \mathrm{E}-5$ & $1.196 \mathrm{E}-4$ & $1.786 \mathrm{E}-2$ & $8.660 \mathrm{E}-6$ & $1.133 \mathrm{E}-4$ & $5.710 \mathrm{E}-5$ & $3.736 \mathrm{E}-5$ & 2.372E-5 & $6.512 \mathrm{E}-9$ & $8.111 \mathrm{E}-8$ & $2.090 \mathrm{E}-3$ \\
\hline
\end{tabular}


Table 4.11. Isotopic concentration in pin 29 vs burnup for Variant 11, State 1

\begin{tabular}{|c|c|c|c|c|c|c|c|c|c|c|c|}
\hline \multirow{2}{*}{$\begin{array}{l}\text { Burnup } \\
\text { (MWd/kg) }\end{array}$} & \multicolumn{11}{|c|}{ Concentration (atoms $/ \mathrm{b} \cdot \mathrm{cm}$, in fuel) } \\
\hline & ${ }^{235} \mathrm{U}$ & ${ }^{236} \mathrm{U}$ & ${ }^{238} \mathrm{U}$ & ${ }^{238} \mathrm{Pu}$ & ${ }^{239} \mathrm{Pu}$ & ${ }^{240} \mathrm{Pu}$ & ${ }^{241} \mathrm{Pu}$ & ${ }^{242} \mathrm{Pu}$ & ${ }^{135} \mathrm{Xe}$ & ${ }^{49} \mathrm{Sm}$ & Total FP \\
\hline$\overline{0}$ & $8.737 \mathrm{E}-4$ & 0.0 & $1.874 \mathrm{E}-2$ & 0.0 & 0.0 & 0.0 & $\overline{0.0}$ & 0.0 & $9.458 \mathrm{E}-9$ & $7.367 \mathrm{E}-8$ & $8.312 \mathrm{E}-8$ \\
\hline 2 & $8.287 \mathrm{E}-4$ & 8.398E-6 & $1.872 \mathrm{E}-2$ & $1.261 \mathrm{E}-9$ & $1.583 \mathrm{E}-5$ & 4.663E-7 & $3.760 \mathrm{E}-8$ & $3.625 \mathrm{E}-10$ & $9.676 \mathrm{E}-9$ & $7.658 \mathrm{E}-8$ & \\
\hline 4 & $7.861 \mathrm{E}-4$ & $1.632 \mathrm{E}-5$ & $1.870 \mathrm{E}-2$ & 7.882E-9 & 3.045E-5 & $1.724 \mathrm{E}-6$ & $2.821 \mathrm{E}-7$ & $5.676 \mathrm{E}-9$ & $9.745 \mathrm{E}-9$ & $8.128 \mathrm{E}-8$ & -4 \\
\hline 6 & $7.455 \mathrm{E}-4$ & 2.383E-5 & $1.868 \mathrm{E}-2$ & $2.252 \mathrm{E}-8$ & $4.306 \mathrm{E}-5$ & 3.470E-6 & 8.316E-7 & $2.599 \mathrm{E}-8$ & $9.774 \mathrm{E}-9$ & 8.517E-8 & $1.919 \mathrm{E}-4$ \\
\hline 8 & $7.067 \mathrm{E}-4$ & $3.095 \mathrm{E}-5$ & $1.866 \mathrm{E}-2$ & $4.744 \mathrm{E}-8$ & $5.397 \mathrm{E}-5$ & $5.523 \mathrm{E}-6$ & $1.695 \mathrm{E}-6$ & $7.289 \mathrm{E}-8$ & 9.771E-9 & $8.844 \mathrm{E}-8$ & $2.562 \mathrm{E}-4$ \\
\hline 10 & $6.695 \mathrm{E}-4$ & 3.772E-5 & $1.863 \mathrm{E}-2$ & 8.473E-8 & $6.346 \mathrm{E}-5$ & 7.773E-6 & $2.839 \mathrm{E}-6$ & $1.572 \mathrm{E}-7$ & $9.744 \mathrm{E}-9$ & $9.116 \mathrm{E}-8$ & $3.207 \mathrm{E}-4$ \\
\hline 12 & $6.339 \mathrm{E}-4$ & $4.415 \mathrm{E}-5$ & $1.861 \mathrm{E}-2$ & $1.363 \mathrm{E}-7$ & 7.172E-5 & $1.015 \mathrm{E}-5$ & $4.216 \mathrm{E}-6$ & $2.881 \mathrm{E}-7$ & $9.698 \mathrm{E}-9$ & $9.342 \mathrm{E}-8$ & $2 \mathrm{E}-4$ \\
\hline 14 & $5.997 \mathrm{E}-4$ & $5.026 \mathrm{E}-5$ & $1.859 \mathrm{E}-2$ & $2.041 \mathrm{E}-7$ & 7.8 & E-5 & $5.774 \mathrm{E}-6$ & $4.728 \mathrm{E}-7$ & 9.637E-9 & $9.525 \mathrm{E}-8$ & E-4 \\
\hline 16 & $5.668 \mathrm{E}-4$ & $5.606 \mathrm{E}-5$ & $1.857 \mathrm{E}-2$ & $2.896 \mathrm{E}-7$ & $8.520 \mathrm{E}-5$ & $1.511 \mathrm{E}-5$ & $7.467 \mathrm{E}-6$ & $5 E-7$ & 9.563E-9 & $9.671 \mathrm{E}-8$ & $E-4$ \\
\hline 18 & $5.353 \mathrm{E}-4$ & $6.157 \mathrm{E}-5$ & $1.854 \mathrm{E}-2$ & $3.946 \mathrm{E}-7$ & $9.066 \mathrm{E}-5$ & $1.763 \mathrm{E}-5$ & $9.252 \mathrm{E}-6$ & $1.023 \mathrm{E}-6$ & $9.478 \mathrm{E}-9$ & $E-8$ & $E-4$ \\
\hline 20 & $5.050 \mathrm{E}-4$ & $6.678 \mathrm{E}-5$ & $1.852 \mathrm{E}-2$ & $5.203 \mathrm{E}-7$ & $9.540 \mathrm{E}-5$ & $2.015 \mathrm{E}-5$ & $1.109 \mathrm{E}-5$ & 1.395E-6 & 9.384E-9 & $9.865 \mathrm{E}-8$ & E-4 \\
\hline 22 & $4.759 \mathrm{E}-4$ & $7.172 \mathrm{E}-5$ & $1.849 \mathrm{E}-2$ & $6.682 \mathrm{E}-7$ & $9.950 \mathrm{E}-5$ & E-5 & $1.296 \mathrm{E}-5$ & $1.833 \mathrm{E}-6$ & $9.283 \mathrm{E}-9$ & $9.919 \mathrm{E}-8$ & $7.089 \mathrm{E}-4$ \\
\hline 24 & $4.480 \mathrm{E}-4$ & $7.638 \mathrm{E}-5$ & $1.846 \mathrm{E}-2$ & 8.393E-7 & $1.030 \mathrm{E}-4$ & $2.514 \mathrm{E}-5$ & 1.4 & $2.338 \mathrm{E}-6$ & 9.174E-9 & $9.952 \mathrm{E}-8$ & $8 \mathrm{E}-4$ \\
\hline 26 & $4.212 \mathrm{E}-4$ & $8.077 \mathrm{E}-5$ & $1.844 \mathrm{E}-2$ & $1.035 \mathrm{E}-6$ & 1.0 & E-5 & 7E-5 & $2.909 \mathrm{E}-6$ & $9.060 \mathrm{E}-9$ & $9.962 \mathrm{E}-8$ & $9 \mathrm{E}-4$ \\
\hline 28 & 3.955E-4 & $8.491 \mathrm{E}-5$ & $1.841 \mathrm{E}-2$ & $1.255 \mathrm{E}-6$ & 1.0 & E-5 & E-5 & $3.544 \mathrm{E}-6$ & 8.940E-9 & $3 \mathrm{E}-8$ & E-4 \\
\hline 30 & 3.709E-4 & 8.879E-5 & $1.838 \mathrm{E}-2$ & $1.501 \mathrm{E}-6$ & 1.1 & 3.2 & E-5 & 43E-6 & 8.817E-9 & $9.927 \mathrm{E}-8$ & E-4 \\
\hline 32 & $3.474 \mathrm{E}-4$ & $9.242 \mathrm{E}-5$ & $1.836 \mathrm{E}-2$ & $1.773 \mathrm{E}-6$ & $1.126 \mathrm{E}-4$ & $3.459 \mathrm{E}-5$ & $2.191 \mathrm{E}-5$ & $5.002 E-6$ & 8.691E-9 & $9.886 \mathrm{E}-8$ & E-3 \\
\hline 34 & $3.249 \mathrm{E}-4$ & $9.581 \mathrm{E}-5$ & $1.833 \mathrm{E}-2$ & $2.071 \mathrm{E}-6$ & $1.141 \mathrm{E}-4$ & $3.680 \mathrm{E}-5$ & $2.353 \mathrm{E}-5$ & $5.820 \mathrm{E}-6$ & $8.562 \mathrm{E}-9$ & $9.833 \mathrm{E}-8$ & E-3 \\
\hline 36 & $3.034 \mathrm{E}-4$ & $9.896 \mathrm{E}-5$ & $1.830 \mathrm{E}-2$ & 2.395E-6 & $1.154 \mathrm{E}-4$ & 3.893E-5 & $2.506 \mathrm{E}-5$ & $6.693 \mathrm{E}-6$ & 8.432E-9 & $9.769 \mathrm{E}-8$ & 65E-3 \\
\hline 38 & $2.829 \mathrm{E}-4$ & $1.019 \mathrm{E}-4$ & $1.827 \mathrm{E}-2$ & $2.745 \mathrm{E}-6$ & $1.163 \mathrm{E}-4$ & $4.099 \mathrm{E}-5$ & $2.651 \mathrm{E}-5$ & $7.618 \mathrm{E}-6$ & $8.300 \mathrm{E}-9$ & $9.695 \mathrm{E}-8$ & $1.230 \mathrm{E}-3$ \\
\hline 40 & $2.633 \mathrm{E}-4$ & $1.046 \mathrm{E}-4$ & $1.824 \mathrm{E}-2$ & $3.120 \mathrm{E}-6$ & $1.171 \mathrm{E}-4$ & $4.296 \mathrm{E}-5$ & $2.788 \mathrm{E}-5$ & $8.592 \mathrm{E}-6$ & $8.168 \mathrm{E}-9$ & $9.614 \mathrm{E}-8$ & $1.296 \mathrm{E}-3$ \\
\hline 42 & $2.448 \mathrm{E}-4$ & $1.070 \mathrm{E}-4$ & $1.821 \mathrm{E}-2$ & 3.519E-6 & 1.1 & $E-5$ & -5 & $9.612 \mathrm{E}-6$ & 8.037E-9 & $9.526 \mathrm{E}-8$ & $1.361 \mathrm{E}-3$ \\
\hline 44 & $2.271 \mathrm{E}-4$ & 1.093 & $1.818 \mathrm{E}-2$ & $2 \mathrm{E}-6$ & & & -5 & E-5 & $7.906 \mathrm{E}-9$ & $9.434 \mathrm{E}-8$ & $1.427 \mathrm{E}-3$ \\
\hline 46 & $2.104 \mathrm{E}-4$ & $1.113 \mathrm{E}-4$ & $1.815 \mathrm{E}-2$ & $4.388 \mathrm{E}-6$ & $1.183 \mathrm{E}-4$ & $4.838 \mathrm{E}-5$ & $3.144 \mathrm{E}-5$ & $1.177 \mathrm{E}-5$ & $7.777 \mathrm{E}-9$ & $9.337 \mathrm{E}-8$ & $1.492 \mathrm{E}-3$ \\
\hline 48 & $1.946 \mathrm{E}-4$ & $1.131 \mathrm{E}-4$ & $1.812 \mathrm{E}-2$ & $4.854 \mathrm{E}-6$ & $1.185 \mathrm{E}-4$ & 5.001E-5 & 3.245E-5 & $1.291 \mathrm{E}-5$ & $7.650 \mathrm{E}-9$ & $9.239 \mathrm{E}-8$ & $1.558 \mathrm{E}-3$ \\
\hline 50 & $1.797 \mathrm{E}-4$ & $1.147 \mathrm{E}-4$ & $1.809 \mathrm{E}-2$ & $5.340 \mathrm{E}-6$ & $1.185 \mathrm{E}-4$ & 5.155E-5 & 3.337E-5 & $1.407 \mathrm{E}-5$ & $7.525 E-9$ & $9.138 \mathrm{E}-8$ & $1.624 \mathrm{E}-3$ \\
\hline 52 & $1.656 \mathrm{E}-4$ & $1.161 \mathrm{E}-4$ & $1.806 \mathrm{E}-2$ & $5.843 \mathrm{E}-6$ & $1.185 \mathrm{E}-4$ & 5.301E-5 & 3.422E-5 & $1.526 \mathrm{E}-5$ & 7.404E-9 & $9.038 \mathrm{E}-8$ & $1.689 \mathrm{E}-3$ \\
\hline 54 & $1.524 \mathrm{E}-4$ & $1.173 \mathrm{E}-4$ & $1.802 \mathrm{E}-2$ & $6.362 \mathrm{E}-6$ & $1.183 \mathrm{E}-4$ & $5.438 \mathrm{E}-5$ & 3.499E-5 & $1.647 \mathrm{E}-5$ & $7.286 \mathrm{E}-9$ & $8.938 \mathrm{E}-8$ & $1.755 \mathrm{E}-3$ \\
\hline 56 & $1.400 \mathrm{E}-4$ & $1.184 \mathrm{E}-4$ & $1.799 \mathrm{E}-2$ & $6.894 \mathrm{E}-6$ & $1.181 \mathrm{E}-4$ & $5.566 \mathrm{E}-5$ & $3.568 \mathrm{E}-5$ & $1.770 \mathrm{E}-5$ & 7.172E-9 & $8.840 \mathrm{E}-8$ & $1.821 \mathrm{E}-3$ \\
\hline 58 & $1.284 \mathrm{E}-4$ & $1.192 \mathrm{E}-4$ & $1.796 \mathrm{E}-2$ & $7.437 \mathrm{E}-6$ & $1.179 \mathrm{E}-4$ & 5.685E-5 & 3.630E-5 & $1.895 \mathrm{E}-5$ & 7.063E-9 & $8.744 \mathrm{E}-8$ & $1.886 \mathrm{E}-3$ \\
\hline 60 & $1.176 \mathrm{E}-4$ & $1.199 \mathrm{E}-4$ & $.792 \mathrm{E}-2$ & $89 \mathrm{E}-6$ & $1.176 \mathrm{E}-4$ & $5.796 \mathrm{E}-5$ & $3.686 \mathrm{E}-5$ & $2.021 \mathrm{E}-5$ & $6.958 \mathrm{E}-9$ & $8.650 \mathrm{E}-8$ & $1.952 \mathrm{E}-3$ \\
\hline
\end{tabular}


Table 4.12. Isotopic concentration in pin 72 vs burnup for Variant 11, State 1

\begin{tabular}{|c|c|c|c|c|c|c|c|c|c|c|c|}
\hline \multirow{2}{*}{$\begin{array}{l}\text { Burnup } \\
\text { (MWd/kg) }\end{array}$} & \multicolumn{11}{|c|}{ Concentration (atoms $/ \mathrm{b} \cdot \mathrm{cm}$, in fuel) } \\
\hline & ${ }^{235} \mathrm{U}$ & ${ }^{236} \mathrm{U}$ & ${ }^{238} \mathrm{U}$ & ${ }^{238} \mathrm{Pu}$ & ${ }^{239} \mathrm{Pu}$ & ${ }^{240} \mathrm{Pu}$ & ${ }^{221} \mathrm{Pu}$ & ${ }^{242} \mathrm{Pu}$ & ${ }^{135} \mathrm{Xe}$ & ${ }^{149} \mathrm{Sm}$ & Total FP \\
\hline 0 & $8.737 \mathrm{E}-4$ & 0.0 & $1.874 \mathrm{E}-2$ & 0.0 & 0.0 & 0.0 & 0.0 & 0.0 & $9.458 \mathrm{E}-9$ & $7.367 \mathrm{E}-8$ & $8.312 \mathrm{E}-8$ \\
\hline 2 & $8.259 \mathrm{E}-4$ & 8.833E-6 & $1.872 \mathrm{E}-2$ & $1.318 \mathrm{E}-9$ & $1.646 \mathrm{E}-5$ & 5.108E-7 & $4.201 E-8$ & 4.307E-10 & 9.697E-9 & $7.519 \mathrm{E}-8$ & $6.784 \mathrm{E}-5$ \\
\hline 4 & $7.808 \mathrm{E}-4$ & $1.714 \mathrm{E}-5$ & $1.870 \mathrm{E}-2$ & 8.317E-9 & $3.154 \mathrm{E}-5$ & $1.881 \mathrm{E}-6$ & $3.136 \mathrm{E}-7$ & $6.726 \mathrm{E}-9$ & $9.754 \mathrm{E}-9$ & $7.969 \mathrm{E}-8$ & $1.355 \mathrm{E}-4$ \\
\hline 6 & 7.378E-4 & $2.499 \mathrm{E}-5$ & $1.868 \mathrm{E}-2$ & 2.391E-8 & 4.439E-5 & $3.776 \mathrm{E}-6$ & $9.201 \mathrm{E}-7$ & $3.071 \mathrm{E}-8$ & $9.766 \mathrm{E}-9$ & $8.348 \mathrm{E}-8$ & $2.036 \mathrm{E}-4$ \\
\hline 8 & $6.969 \mathrm{E}-4$ & $3.242 \mathrm{E}-5$ & $1.865 \mathrm{E}-2$ & $5.059 \mathrm{E}-8$ & $5.540 \mathrm{E}-5$ & $5.989 \mathrm{E}-6$ & $1.867 \mathrm{E}-6$ & $8.594 \mathrm{E}-8$ & 9.744E-9 & 8.663E-8 & $2.717 \mathrm{E}-4$ \\
\hline 10 & $6.578 \mathrm{E}-4$ & $3.945 \mathrm{E}-5$ & $1.863 \mathrm{E}-2$ & 9.064E-8 & $6.486 \mathrm{E}-5$ & 8.399E-6 & $3.114 \mathrm{E}-6$ & $1.849 \mathrm{E}-7$ & $9.697 \mathrm{E}-9$ & 8.923E-8 & 3.399E-4 \\
\hline 12 & $6.204 \mathrm{E}-4$ & $4.610 \mathrm{E}-5$ & $1.861 \mathrm{E}-2$ & $1.462 \mathrm{E}-7$ & $7.302 \mathrm{E}-5$ & $1.093 \mathrm{E}-5$ & $4.604 \mathrm{E}-6$ & $3.381 \mathrm{E}-7$ & $9.630 \mathrm{E}-9$ & $9.134 \mathrm{E}-8$ & $4.080 \mathrm{E}-4$ \\
\hline 14 & $5.847 \mathrm{E}-4$ & $5.240 \mathrm{E}-5$ & $1.858 \mathrm{E}-2$ & $2.192 \mathrm{E}-7$ & $8.004 \mathrm{E}-5$ & $1.353 \mathrm{E}-5$ & $6.281 \mathrm{E}-6$ & 5.534E-7 & $9.547 \mathrm{E}-9$ & $9.303 \mathrm{E}-8$ & $4.762 \mathrm{E}-4$ \\
\hline 16 & $5.504 \mathrm{E}-4$ & $5.835 \mathrm{E}-5$ & $1.856 \mathrm{E}-2$ & $3.114 \mathrm{E}-7$ & $8.609 \mathrm{E}-5$ & $1.615 \mathrm{E}-5$ & $8.089 \mathrm{E}-6$ & 8.367E-7 & $9.452 \mathrm{E}-9$ & $9.434 \mathrm{E}-8$ & $5.443 \mathrm{E}-4$ \\
\hline 18 & $5.177 \mathrm{E}-4$ & $6.398 \mathrm{E}-5$ & $1.853 \mathrm{E}-2$ & $4.245 \mathrm{E}-7$ & $9.129 \mathrm{E}-5$ & $1.878 \mathrm{E}-5$ & $9.982 \mathrm{E}-6$ & $1.192 \mathrm{E}-6$ & 9.347E-9 & $9.531 \mathrm{E}-8$ & $6.124 \mathrm{E}-4$ \\
\hline 20 & $4.863 \mathrm{E}-4$ & $6.928 \mathrm{E}-5$ & $1.851 \mathrm{E}-2$ & 5.601E-7 & 9.573E-5 & $2.139 \mathrm{E}-5$ & $1.192 \mathrm{E}-5$ & $1.621 \mathrm{E}-6$ & $9.232 \mathrm{E}-9$ & $9.599 \mathrm{E}-8$ & $6.805 \mathrm{E}-4$ \\
\hline 22 & $4.563 \mathrm{E}-4$ & $7.428 \mathrm{E}-5$ & $1.848 \mathrm{E}-2$ & 7.193E-7 & 9.953E-5 & 2.397E-5 & $1.387 \mathrm{E}-5$ & $2.125 \mathrm{E}-6$ & $9.110 \mathrm{E}-9$ & $9.639 \mathrm{E}-8$ & $7.485 \mathrm{E}-4$ \\
\hline 24 & $4.276 \mathrm{E}-4$ & $7.897 \mathrm{E}-5$ & $1.845 \mathrm{E}-2$ & $9.035 \mathrm{E}-7$ & $1.028 \mathrm{E}-4$ & $2.650 \mathrm{E}-5$ & $1.580 \mathrm{E}-5$ & $2.703 \mathrm{E}-6$ & $8.982 \mathrm{E}-9$ & $9.656 \mathrm{E}-8$ & $8.165 \mathrm{E}-4$ \\
\hline 26 & $4.002 \mathrm{E}-4$ & 8.337E-5 & $1.843 \mathrm{E}-2$ & $1.113 \mathrm{E}-6$ & $1.055 \mathrm{E}-4$ & $2.897 \mathrm{E}-5$ & $1.770 \mathrm{E}-5$ & 3.355E-6 & $8.850 \mathrm{E}-9$ & $9.652 \mathrm{E}-8$ & $8.845 \mathrm{E}-4$ \\
\hline 28 & $3.741 \mathrm{E}-4$ & $8.749 \mathrm{E}-5$ & $1.840 \mathrm{E}-2$ & $1.350 \mathrm{E}-6$ & $1.077 \mathrm{E}-4$ & E-5 & $1.953 \mathrm{E}-5$ & 4.078E-6 & 8.713E-9 & $9.629 \mathrm{E}-8$ & $9.524 \mathrm{E}-4$ \\
\hline 30 & $3.491 \mathrm{E}-4$ & $9.133 \mathrm{E}-5$ & $1.837 \mathrm{E}-2$ & $1.613 \mathrm{E}-6$ & $1.096 \mathrm{E}-4$ & 9E-5 & $2.130 \mathrm{E}-5$ & $4.869 \mathrm{E}-6$ & $8.573 \mathrm{E}-9$ & $9.590 \mathrm{E}-8$ & $1.020 \mathrm{E}-3$ \\
\hline 32 & $3.253 \mathrm{E}-4$ & $9.489 \mathrm{E}-5$ & $1.834 \mathrm{E}-2$ & $1.903 \mathrm{E}-6$ & $1.112 \mathrm{E}-4$ & 3.593E-5 & $2.299 \mathrm{E}-5$ & $5.725 \mathrm{E}-6$ & $8.432 \mathrm{E}-9$ & $9.538 \mathrm{E}-8$ & $1.088 \mathrm{E}-3$ \\
\hline 34 & $3.027 \mathrm{E}-4$ & $9.819 \mathrm{E}-5$ & $1.831 \mathrm{E}-2$ & $2.221 \mathrm{E}-6$ & $1.124 \mathrm{E}-4$ & $3.809 \mathrm{E}-5$ & $2.459 \mathrm{E}-5$ & $6.643 \mathrm{E}-6$ & $8.289 \mathrm{E}-9$ & $9.473 \mathrm{E}-8$ & $1.156 \mathrm{E}-3$ \\
\hline 36 & $2.812 \mathrm{E}-4$ & $1.012 \mathrm{E}-4$ & $1.828 \mathrm{E}-2$ & $2.565 \mathrm{E}-6$ & $1.134 \mathrm{E}-4$ & $4.015 \mathrm{E}-5$ & $2.609 \mathrm{E}-5$ & $7.619 \mathrm{E}-6$ & $8.146 \mathrm{E}-9$ & $9.398 \mathrm{E}-8$ & $1.224 \mathrm{E}-3$ \\
\hline 38 & $2.608 \mathrm{E}-4$ & $1.040 \mathrm{E}-4$ & $1.825 \mathrm{E}-2$ & $2.936 \mathrm{E}-6$ & $1.141 \mathrm{E}-4$ & $4.213 \mathrm{E}-5$ & $2.750 \mathrm{E}-5$ & 8.649E-6 & $8.003 \mathrm{E}-9$ & $9.315 \mathrm{E}-8$ & $1.291 \mathrm{E}-3$ \\
\hline 40 & $2.414 \mathrm{E}-4$ & $1.066 \mathrm{E}-4$ & $1.822 \mathrm{E}-2$ & 3.332E-6 & $1.146 \mathrm{E}-4$ & $0 \mathrm{E}-5$ & $2.881 \mathrm{E}-5$ & $9.728 \mathrm{E}-6$ & $7.861 \mathrm{E}-9$ & $9.226 \mathrm{E}-8$ & $1.359 \mathrm{E}-3$ \\
\hline 42 & $2.231 \mathrm{E}-4$ & $1.089 \mathrm{E}-4$ & $1.819 \mathrm{E}-2$ & $3 \mathrm{E}-6$ & 1. & -5 & -5 & $1.085 \mathrm{E}-5$ & $7.721 \mathrm{E}-9$ & $9.130 \mathrm{E}-8$ & $1.427 \mathrm{E}-3$ \\
\hline 44 & $2.059 \mathrm{E}-4$ & $1.109 \mathrm{E}-4$ & $1.816 \mathrm{E}-2$ & 4.197E-6 & $1.152 \mathrm{E}-4$ & $4.747 \mathrm{E}-5$ & $3.112 \mathrm{E}-5$ & $1.202 \mathrm{E}-5$ & $7.584 \mathrm{E}-9$ & $9.031 \mathrm{E}-8$ & $1.494 \mathrm{E}-3$ \\
\hline 46 & $1.896 \mathrm{E}-4$ & $1.128 \mathrm{E}-4$ & $1.813 \mathrm{E}-2$ & $4.663 \mathrm{E}-6$ & $1.154 \mathrm{E}-4$ & $4.906 \mathrm{E}-5$ & $3.214 \mathrm{E}-5$ & $1.322 \mathrm{E}-5$ & $7.449 \mathrm{E}-9$ & $8.930 \mathrm{E}-8$ & $1.562 \mathrm{E}-3$ \\
\hline 48 & $1.743 \mathrm{E}-4$ & $1.144 \mathrm{E}-4$ & $1.810 \mathrm{E}-2$ & $5.148 \mathrm{E}-6$ & $1.154 \mathrm{E}-4$ & 5.055E-5 & 3.306E-5 & 1.445E-5 & $7.318 \mathrm{E}-9$ & 8.827E-8 & $1.629 \mathrm{E}-3$ \\
\hline 50 & $1.599 \mathrm{E}-4$ & $1.158 \mathrm{E}-4$ & $1.806 \mathrm{E}-2$ & $5.652 \mathrm{E}-6$ & $1.153 \mathrm{E}-4$ & 5.195E-5 & $3.390 \mathrm{E}-5$ & $1.571 \mathrm{E}-5$ & $7.190 \mathrm{E}-9$ & $8.724 \mathrm{E}-8$ & $1.697 \mathrm{E}-3$ \\
\hline 52 & $1.465 \mathrm{E}-4$ & $1.170 \mathrm{E}-4$ & $1.803 \mathrm{E}-2$ & $6.171 \mathrm{E}-6$ & $1.151 \mathrm{E}-4$ & $5.325 \mathrm{E}-5$ & $3.465 E-5$ & $1.700 \mathrm{E}-5$ & $7.068 \mathrm{E}-9$ & $8.621 \mathrm{E}-8$ & $1.764 \mathrm{E}-3$ \\
\hline 54 & $1.339 \mathrm{E}-4$ & $1.180 \mathrm{E}-4$ & $1.800 \mathrm{E}-2$ & $6.704 \mathrm{E}-6$ & $1.149 \mathrm{E}-4$ & $5.446 \mathrm{E}-5$ & 3.533E-5 & $1.830 \mathrm{E}-5$ & $6.949 \mathrm{E}-9$ & $8.520 \mathrm{E}-8$ & $1.832 \mathrm{E}-3$ \\
\hline 56 & $1.222 \mathrm{E}-4$ & $1.188 \mathrm{E}-4$ & $1.796 \mathrm{E}-2$ & $7.248 \mathrm{E}-6$ & $1.147 \mathrm{E}-4$ & $5.559 \mathrm{E}-5$ & 3.593E-5 & $1.961 \mathrm{E}-5$ & $6.837 \mathrm{E}-9$ & $8.422 \mathrm{E}-8$ & $1.899 \mathrm{E}-3$ \\
\hline 58 & $1.114 \mathrm{E}-4$ & $1.194 \mathrm{E}-4$ & $1.793 \mathrm{E}-2$ & $7.800 \mathrm{E}-6$ & $1.144 \mathrm{E}-4$ & 5.663E-5 & $3.646 \mathrm{E}-5$ & 2.094E-5 & $6.729 \mathrm{E}-9$ & $8.326 \mathrm{E}-8$ & $1.967 \mathrm{E}-3$ \\
\hline 60 & $1.013 \mathrm{E}-4$ & $1.199 \mathrm{E}-4$ & $1.789 \mathrm{E}-2$ & 8.359E-6 & $1.141 \mathrm{E}-4$ & $5.758 \mathrm{E}-5$ & 3.693E-5 & $2.227 \mathrm{E}-5$ & $6.628 \mathrm{E}-9$ & $8.235 \mathrm{E}-8$ & $2.034 E-3$ \\
\hline
\end{tabular}


Table 4.13. Isotopic concentration in pin 1 vs burnup for Variant 12, State 1

\begin{tabular}{|c|c|c|c|c|c|c|c|c|c|c|c|}
\hline \multirow{2}{*}{$\begin{array}{c}\text { Burnup } \\
\text { (MWd/kg) }\end{array}$} & \multicolumn{11}{|c|}{ Concentration (atoms $/ \mathrm{b} \cdot \mathrm{cm}$, in fuel) } \\
\hline & ${ }^{235} \mathrm{U}$ & ${ }^{236} \mathrm{U}$ & ${ }^{238} \mathrm{U}$ & ${ }^{238} \mathrm{Pu}$ & ${ }^{239} \mathrm{Pu}$ & ${ }^{240} \mathrm{Pu}$ & ${ }^{221} \mathrm{Pu}$ & ${ }^{242} \mathrm{Pu}$ & ${ }^{135} \mathrm{Xe}$ & ${ }^{149} \mathrm{Sm}$ & Total FP \\
\hline 0 & $3.839 \mathrm{E}-5$ & 0.0 & $1.892 \mathrm{E}-2$ & $\overline{0.0}$ & $6.588 \mathrm{E}-4$ & $4.232 \mathrm{E}-5$ & $7.025 \mathrm{E}-6$ & 0.0 & $9.458 \mathrm{E}-9$ & $7.367 \mathrm{E}-8$ & $8.312 \mathrm{E}-8$ \\
\hline 2 & 3.693E-5 & $3.132 \mathrm{E}-7$ & $1.889 \mathrm{E}-2$ & $1.425 \mathrm{E}-9$ & $6.180 \mathrm{E}-4$ & $5.708 \mathrm{E}-5$ & $1.262 \mathrm{E}-5$ & $2.268 \mathrm{E}-7$ & $1.660 \mathrm{E}-8$ & $1.491 \mathrm{E}-7$ & 5 \\
\hline 4 & $3.549 \mathrm{E}-5$ & $6.204 \mathrm{E}-7$ & $1.887 \mathrm{E}-2$ & $7.903 E-9$ & $5.803 \mathrm{E}-4$ & $7.035 \mathrm{E}-5$ & $1.866 \mathrm{E}-5$ & $5.964 \mathrm{E}-7$ & $1.611 \mathrm{E}-8$ & $1.541 \mathrm{E}-7$ & 1.42 \\
\hline 6 & $3.405 \mathrm{E}-5$ & $9.207 \mathrm{E}-7$ & $1.884 \mathrm{E}-2$ & $2.070 \mathrm{E}-8$ & $5.443 E-4$ & 8.227E-5 & $2.487 \mathrm{E}-5$ & $1.122 \mathrm{E}-6$ & $1.562 \mathrm{E}-8$ & $1.535 \mathrm{E}-7$ & 2.237E-4 \\
\hline 8 & $3.262 \mathrm{E}-5$ & $1.213 \mathrm{E}-6$ & $1.882 \mathrm{E}-2$ & $4.097 \mathrm{E}-8$ & $5.101 \mathrm{E}-4$ & $9.294 \mathrm{E}-5$ & 3.109E-5 & $1.812 \mathrm{E}-6$ & $1.513 \mathrm{E}-8$ & $1.525 \mathrm{E}-7$ & $2.978 \mathrm{E}-4$ \\
\hline 10 & $3.121 \mathrm{E}-5$ & $1.498 \mathrm{E}-6$ & $1.879 \mathrm{E}-2$ & $6.998 \mathrm{E}-8$ & $4.776 \mathrm{E}-4$ & $1.024 \mathrm{E}-4$ & $3.718 \mathrm{E}-5$ & $2.670 \mathrm{E}-6$ & $1.467 \mathrm{E}-8$ & $1.511 \mathrm{E}-7$ & $3.716 \mathrm{E}-4$ \\
\hline 12 & $2.982 \mathrm{E}-5$ & $1.774 \mathrm{E}-6$ & $1.876 \mathrm{E}-2$ & $1.091 \mathrm{E}-7$ & $4.469 \mathrm{E}-4$ & $1.108 \mathrm{E}-4$ & $4.304 \mathrm{E}-5$ & $3.700 \mathrm{E}-6$ & $1.421 \mathrm{E}-8$ & $1.494 \mathrm{E}-7$ & 4.449E-4 \\
\hline 14 & $2.845 \mathrm{E}-5$ & $2.042 \mathrm{E}-6$ & $1.874 \mathrm{E}-2$ & $1.595 \mathrm{E}-7$ & -4 & E-4 & 4.85 & $4.900 \mathrm{E}-6$ & $1.376 \mathrm{E}-8$ & $1.474 \mathrm{E}-7$ & $0 \mathrm{E}-4$ \\
\hline 16 & $2.710 \mathrm{E}-5$ & $2.301 \mathrm{E}-6$ & 1.871E-2 & $2.227 \mathrm{E}-7$ & 3.907E-4 & $1.243 \mathrm{E}-4$ & $5.378 \mathrm{E}-5$ & $6.270 \mathrm{E}-6$ & $1.333 \mathrm{E}-8$ & $1.452 \mathrm{E}-7$ & E-4 \\
\hline 18 & $2.577 \mathrm{E}-5$ & $2.550 \mathrm{E}-6$ & $1.868 \mathrm{E}-2$ & 2.996E-7 & $3.654 \mathrm{E}-4$ & $1.296 \mathrm{E}-4$ & $5.856 \mathrm{E}-5$ & $7.805 \mathrm{E}-6$ & $1.291 \mathrm{E}-8$ & $1.428 \mathrm{E}-7$ & OE-4 \\
\hline 20 & $2.447 \mathrm{E}-5$ & $2.790 \mathrm{E}-6$ & $1.865 \mathrm{E}-2$ & $3.915 \mathrm{E}-7$ & $3.417 \mathrm{E}-4$ & $1.339 \mathrm{E}-4$ & $6.289 \mathrm{E}-5$ & $9.499 \mathrm{E}-6$ & $1.250 \mathrm{E}-8$ & $1.403 \mathrm{E}-7$ & 7.349E-4 \\
\hline 22 & $2.320 \mathrm{E}-5$ & $3.020 \mathrm{E}-6$ & $1.862 \mathrm{E}-2$ & $4.990 \mathrm{E}-7$ & $3.198 \mathrm{E}-4$ & $1.373 \mathrm{E}-4$ & $6.675 \mathrm{E}-5$ & $1.135 \mathrm{E}-5$ & $1.211 \mathrm{E}-8$ & $1.377 \mathrm{E}-7$ & $8.066 \mathrm{E}-4$ \\
\hline 24 & $2.195 \mathrm{E}-5$ & $3.240 \mathrm{E}-6$ & $1.859 \mathrm{E}-2$ & $6.228 \mathrm{E}-7$ & $2.996 \mathrm{E}-4$ & $1.399 \mathrm{E}-4$ & 7.01 & 1.3 & $1.174 \mathrm{E}-8$ & $1.351 \mathrm{E}-7$ & $8.780 \mathrm{E}-4$ \\
\hline 26 & 2.074E-5 & $3.449 \mathrm{E}-6$ & $1.856 \mathrm{E}-2$ & $7.632 \mathrm{E}-7$ & -4 & E-4 & 7.3 & -5 & E-8 & $4 \mathrm{E}-7$ & -4 \\
\hline 28 & $1.956 \mathrm{E}-5$ & $3.648 \mathrm{E}-6$ & $1.853 \mathrm{E}-2$ & $9.203 \mathrm{E}-7$ & -4 & E-4 & -5 & -5 & E-8 & E-7 & E-3 \\
\hline 30 & $1.841 \mathrm{E}-5$ & $3.837 \mathrm{E}-6$ & $1.850 \mathrm{E}-2$ & $1.094 \mathrm{E}-6$ & $a-4$ & 1.4 & 7.73 & 2.0 & $1.070 \mathrm{E}-8$ & $1.270 \mathrm{E}-7$ & 1E-3 \\
\hline 32 & $1.730 \mathrm{E}-5$ & $4.014 E-6$ & $1.847 \mathrm{E}-2$ & $1.283 \mathrm{E}-6$ & $2.344 \mathrm{E}-4$ & $1.430 \mathrm{E}-4$ & $7.884 E-5$ & $2.250 \mathrm{E}-5$ & $1.039 \mathrm{E}-8$ & $1.244 \mathrm{E}-7$ & $1 E-3$ \\
\hline 34 & $1.623 \mathrm{E}-5$ & $4.181 \mathrm{E}-6$ & $1.844 \mathrm{E}-2$ & $1.488 \mathrm{E}-6$ & $2.217 \mathrm{E}-4$ & $1.422 \mathrm{E}-4$ & $7.989 \mathrm{E}-5$ & $2.503 \mathrm{E}-5$ & $1.009 \mathrm{E}-8$ & $1.218 \mathrm{E}-7$ & $1.231 \mathrm{E}-3$ \\
\hline 36 & $1.519 \mathrm{E}-5$ & $4.337 \mathrm{E}-6$ & $1.840 \mathrm{E}-2$ & $1.706 \mathrm{E}-6$ & $2.102 \mathrm{E}-4$ & $1.410 \mathrm{E}-4$ & $8.052 \mathrm{E}-5$ & $2.762 \mathrm{E}-5$ & $9.807 E-9$ & $1.193 \mathrm{E}-7$ & $1.301 \mathrm{E}-3$ \\
\hline 38 & $1.420 \mathrm{E}-5$ & $4.482 \mathrm{E}-6$ & $1.837 \mathrm{E}-2$ & $1.937 \mathrm{E}-6$ & $1.999 \mathrm{E}-4$ & $1.394 \mathrm{E}-4$ & $8.079 \mathrm{E}-5$ & $3.026 \mathrm{E}-5$ & 9.540E-9 & $1.168 \mathrm{E}-7$ & $1.371 \mathrm{E}-3$ \\
\hline 40 & $1.324 \mathrm{E}-5$ & $4.616 \mathrm{E}-6$ & $1.834 \mathrm{E}-2$ & $2.179 \mathrm{E}-6$ & E-4 & $1.374 \mathrm{E}-4$ & $8.072 E-5$ & $3.293 \mathrm{E}-5$ & $9.288 \mathrm{E}-9$ & $1.144 \mathrm{E}-7$ & $1.440 \mathrm{E}-3$ \\
\hline 42 & E-5 & -6 & $1.830 \mathrm{E}-2$ & E-6 & & -4 & -5 & & $9.050 \mathrm{E}-9$ & E-7 & $1.510 \mathrm{E}-3$ \\
\hline 44 & 1.1 & $4.851 \mathrm{E}-6$ & $1.827 \mathrm{E}-2$ & $2.688 \mathrm{E}-6$ & 1.7 & -4 & 7.9 & 3.83 & $8.826 \mathrm{E}-9$ & $1.098 \mathrm{E}-7$ & $1.579 \mathrm{E}-3$ \\
\hline 46 & $1.062 \mathrm{E}-5$ & $4.952 \mathrm{E}-6$ & $1.824 \mathrm{E}-2$ & 2.951E-6 & $1.686 \mathrm{E}-4$ & $1.299 \mathrm{E}-4$ & 7.880 E-5 & $4.100 \mathrm{E}-5$ & E-9 & $1.077 \mathrm{E}-7$ & $1.649 \mathrm{E}-3$ \\
\hline 48 & $9.830 \mathrm{E}-6$ & $5.043 \mathrm{E}-6$ & $1.820 \mathrm{E}-2$ & 3.217E-6 & $1.628 \mathrm{E}-4$ & $1.270 \mathrm{E}-4$ & 7.771E-5 & 4.365E-5 & $8.415 \mathrm{E}-9$ & $1.056 \mathrm{E}-7$ & $1.718 \mathrm{E}-3$ \\
\hline 50 & $9.082 \mathrm{E}-6$ & $5.124 \mathrm{E}-6$ & $1.817 \mathrm{E}-2$ & $3.484 \mathrm{E}-6$ & $1.577 \mathrm{E}-4$ & $1.241 \mathrm{E}-4$ & $7.647 \mathrm{E}-5$ & $4.627 \mathrm{E}-5$ & $8.228 \mathrm{E}-9$ & $1.037 \mathrm{E}-7$ & $1.787 \mathrm{E}-3$ \\
\hline 52 & $8.376 \mathrm{E}-6$ & $5.194 \mathrm{E}-6$ & $1.813 \mathrm{E}-2$ & 3.749E-6 & $1.531 \mathrm{E}-4$ & $1.210 \mathrm{E}-4$ & $7.509 \mathrm{E}-5$ & $4.884 \mathrm{E}-5$ & $8.051 \mathrm{E}-9$ & $1.018 \mathrm{E}-7$ & $1.857 \mathrm{E}-3$ \\
\hline 54 & $7.710 \mathrm{E}-6$ & $5.255 \mathrm{E}-6$ & $1.809 \mathrm{E}-2$ & $4.010 \mathrm{E}-6$ & $1.491 \mathrm{E}-4$ & $1.180 \mathrm{E}-4$ & 7.362E-5 & $5.135 \mathrm{E}-5$ & $7.885 \mathrm{E}-9$ & $9.997 \mathrm{E}-8$ & $1.926 \mathrm{E}-3$ \\
\hline 56 & $7.085 \mathrm{E}-6$ & $5.307 \mathrm{E}-6$ & $1.806 \mathrm{E}-2$ & $4.266 \mathrm{E}-6$ & $1.455 \mathrm{E}-4$ & $1.150 \mathrm{E}-4$ & $7.207 \mathrm{E}-5$ & $5.379 \mathrm{E}-5$ & $7.728 \mathrm{E}-9$ & $9.825 \mathrm{E}-8$ & $1.995 \mathrm{E}-3$ \\
\hline 58 & $6.499 \mathrm{E}-6$ & $5.349 \mathrm{E}-6$ & $1.802 \mathrm{E}-2$ & $4.515 \mathrm{E}-6$ & $1.422 \mathrm{E}-4$ & $1.120 \mathrm{E}-4$ & 7.047E-5 & $5.616 \mathrm{E}-5$ & $7.581 \mathrm{E}-9$ & $9.661 \mathrm{E}-8$ & $2.064 \mathrm{E}-3$ \\
\hline 60 & $5.950 \mathrm{E}-6$ & $5.383 \mathrm{E}-6$ & $1.798 \mathrm{E}-2$ & $4.754 \mathrm{E}-6$ & $1.394 \mathrm{E}-4$ & $1.091 \mathrm{E}-4$ & $6.885 \mathrm{E}-5$ & $5.845 \mathrm{E}-5$ & $7.443 \mathrm{E}-9$ & $9.507 \mathrm{E}-8$ & E-3 \\
\hline
\end{tabular}


Table 4.14. Isotopic concentration in pin 29 vs burnup for Variant 12, State 1

\begin{tabular}{|c|c|c|c|c|c|c|c|c|c|c|c|}
\hline \multirow{2}{*}{$\begin{array}{l}\text { Burnup } \\
(\mathrm{MWd} / \mathrm{kg})\end{array}$} & \multicolumn{11}{|c|}{ Concentration (atoms $/ \mathrm{b} \cdot \mathrm{cm}$, in fuel) } \\
\hline & ${ }^{235} \mathrm{U}$ & ${ }^{236} \mathrm{U}$ & ${ }^{238} \mathrm{U}$ & ${ }^{238} \mathrm{Pu}$ & ${ }^{239} \mathrm{Pu}$ & ${ }^{240} \mathrm{Pu}$ & ${ }^{241} \mathrm{Pu}$ & ${ }^{242} \mathrm{Pu}$ & ${ }^{135} \mathrm{Xe}$ & ${ }^{149} \mathrm{Sm}$ & Total FP \\
\hline 0 & $3.839 \mathrm{E}-5$ & 0.0 & $1.892 \mathrm{E}-2$ & 0.0 & $6.588 \mathrm{E}-4$ & $4.232 \mathrm{E}-5$ & $7.025 \mathrm{E}-6$ & 0.0 & $9.458 \mathrm{E}-9$ & $7.367 \mathrm{E}-8$ & $8.312 \mathrm{E}-8$ \\
\hline 2 & $3.712 \mathrm{E}-5$ & $2.842 \mathrm{E}-7$ & $1.889 \mathrm{E}-2$ & $1.312 \mathrm{E}-9$ & $6.259 \mathrm{E}-4$ & $5.456 \mathrm{E}-5$ & $1.196 \mathrm{E}-5$ & $1.861 \mathrm{E}-7$ & $1.593 \mathrm{E}-8$ & $1.521 \mathrm{E}-7$ & $5 \mathrm{E}-5$ \\
\hline 4 & 3.585E-5 & $5.640 \mathrm{E}-7$ & $1.887 \mathrm{E}-2$ & $7.258 \mathrm{E}-9$ & $5.954 \mathrm{E}-4$ & $6.581 \mathrm{E}-5$ & $1.724 \mathrm{E}-5$ & $4.772 \mathrm{E}-7$ & $1.562 \mathrm{E}-8$ & $1.630 \mathrm{E}-7$ & $9 \mathrm{E}-4$ \\
\hline 6 & $3.458 \mathrm{E}-5$ & $8.385 \mathrm{E}-7$ & $1.885 \mathrm{E}-2$ & $1.894 \mathrm{E}-8$ & $5.659 \mathrm{E}-4$ & $7.616 \mathrm{E}-5$ & $2.266 \mathrm{E}-5$ & $8.820 \mathrm{E}-7$ & $1.531 \mathrm{E}-8$ & $1.635 \mathrm{E}-7$ & $1.923 \mathrm{E}-4$ \\
\hline 8 & 3.333E-5 & $1.107 \mathrm{E}-6$ & $1.882 \mathrm{E}-2$ & $3.734 \mathrm{E}-8$ & $5.373 \mathrm{E}-4$ & 8.567E-5 & $2.812 \mathrm{E}-5$ & $1.406 \mathrm{E}-6$ & $1.499 \mathrm{E}-8$ & $1.631 \mathrm{E}-7$ & $2.569 \mathrm{E}-4$ \\
\hline 10 & $3.208 \mathrm{E}-5$ & $1.370 \mathrm{E}-6$ & $1.880 \mathrm{E}-2$ & $6.350 \mathrm{E}-8$ & $5.098 \mathrm{E}-4$ & $9.438 \mathrm{E}-5$ & $3.353 \mathrm{E}-5$ & $2.054 \mathrm{E}-6$ & $1.468 \mathrm{E}-8$ & $1.623 \mathrm{E}-7$ & $3.216 \mathrm{E}-4$ \\
\hline 12 & $3.084 \mathrm{E}-5$ & $1.626 \mathrm{E}-6$ & $1.877 \mathrm{E}-2$ & $9.854 \mathrm{E}-8$ & $4.833 E-4$ & $1.023 \mathrm{E}-4$ & $3.880 \mathrm{E}-5$ & $2.829 \mathrm{E}-6$ & $1.436 \mathrm{E}-8$ & $1.611 \mathrm{E}-7$ & $5 E-4$ \\
\hline 14 & $2.961 \mathrm{E}-5$ & $1.876 \mathrm{E}-6$ & $1.875 \mathrm{E}-2$ & $1.436 \mathrm{E}-7$ & $4.579 \mathrm{E}-4$ & $1.095 \mathrm{E}-4$ & 4.390E-5 & $3.732 \mathrm{E}-6$ & $1.404 \mathrm{E}-8$ & $1.595 \mathrm{E}-7$ & $6 \mathrm{E}-4$ \\
\hline 16 & $2.840 \mathrm{E}-5$ & $2.118 \mathrm{E}-6$ & $1.872 \mathrm{E}-2$ & $1.997 \mathrm{E}-7$ & $4.335 \mathrm{E}-4$ & $1.160 \mathrm{E}-4$ & $4.877 \mathrm{E}-5$ & $4.764 \mathrm{E}-6$ & $1.373 \mathrm{E}-8$ & $1.577 \mathrm{E}-7$ & $8 E-4$ \\
\hline 18 & $2.720 \mathrm{E}-5$ & $2.354 \mathrm{E}-6$ & $1.869 \mathrm{E}-2$ & $2.680 \mathrm{E}-7$ & $4.103 \mathrm{E}-4$ & $1.218 \mathrm{E}-4$ & $5.336 \mathrm{E}-5$ & $5.925 \mathrm{E}-6$ & $1.341 \mathrm{E}-8$ & $1.557 \mathrm{E}-7$ & $1 \mathrm{E}-4$ \\
\hline 20 & $2.602 \mathrm{E}-5$ & $2.582 \mathrm{E}-6$ & $1.867 \mathrm{E}-2$ & 3.494E-7 & $3.881 \mathrm{E}-4$ & $1.268 \mathrm{E}-4$ & $5.765 \mathrm{E}-5$ & 7.213E-6 & $1.310 \mathrm{E}-8$ & $1.534 \mathrm{E}-7$ & $6.476 \mathrm{E}-4$ \\
\hline 22 & $2.485 \mathrm{E}-5$ & 2.802E-6 & $1.864 \mathrm{E}-2$ & 4.447E-7 & $3.671 \mathrm{E}-4$ & 1.312E-4 & $6.161 \mathrm{E}-5$ & 8.627E-6 & $1.279 \mathrm{E}-8$ & $1.511 \mathrm{E}-7$ & $1 \mathrm{E}-4$ \\
\hline 24 & $2.370 \mathrm{E}-5$ & $3.015 \mathrm{E}-6$ & $1.861 \mathrm{E}-2$ & $5.547 \mathrm{E}-7$ & $3.472 \mathrm{E}-4$ & $1.350 \mathrm{E}-4$ & & $1.016 \mathrm{E}-5$ & $1.249 \mathrm{E}-8$ & E-7 & $3 E-4$ \\
\hline 26 & $2.257 \mathrm{E}-5$ & $3.219 \mathrm{E}-6$ & $1.859 \mathrm{E}-2$ & $6.798 \mathrm{E}-7$ & $3.284 \mathrm{E}-4$ & $1.381 \mathrm{E}-4$ & $E-5$ & $1.182 \mathrm{E}-5$ & 9E-8 & E-7 & 6E-4 \\
\hline 28 & $2.147 \mathrm{E}-5$ & $3.416 \mathrm{E}-6$ & $1.856 \mathrm{E}-2$ & 8.204E-7 & $3.108 \mathrm{E}-4$ & $1.407 \mathrm{E}-4$ & $7.134 \mathrm{E}-5$ & $1.358 \mathrm{E}-5$ & 1.189E-8 & $1.433 \mathrm{E}-7$ & 6E-4 \\
\hline 30 & $2.038 \mathrm{E}-5$ & $3.604 \mathrm{E}-6$ & $1.853 \mathrm{E}-2$ & $9.766 \mathrm{E}-7$ & $2.942 \mathrm{E}-4$ & 1.4 & E-5 & $1.546 \mathrm{E}-5$ & $1.160 \mathrm{E}-8$ & $1.406 \mathrm{E}-7$ & E-4 \\
\hline 32 & $1.932 \mathrm{E}-5$ & $3.783 \mathrm{E}-6$ & $1.850 \mathrm{E}-2$ & $1.148 \mathrm{E}-6$ & $2.788 \mathrm{E}-4$ & $1.441 \mathrm{E}-4$ & $7.595 \mathrm{E}-5$ & $1.743 \mathrm{E}-5$ & $1.131 \mathrm{E}-8$ & 1.379E-7 & BE-3 \\
\hline 34 & $1.829 \mathrm{E}-5$ & 3.954E-6 & $1.847 \mathrm{E}-2$ & 1.335E-6 & $2.644 \mathrm{E}-4$ & $1.450 \mathrm{E}-4$ & 7.771E-5 & $1.950 \mathrm{E}-5$ & $1.103 \mathrm{E}-8$ & $1.351 \mathrm{E}-7$ & $1.109 \mathrm{E}-3$ \\
\hline 36 & $1.728 \mathrm{E}-5$ & $4.116 \mathrm{E}-6$ & $1.844 \mathrm{E}-2$ & $1.537 \mathrm{E}-6$ & 2.5 & -4 & & $2.165 \mathrm{E}-5$ & $1.076 \mathrm{E}-8$ & $1.324 \mathrm{E}-7$ & $1.175 \mathrm{E}-3$ \\
\hline 38 & $1.630 \mathrm{E}-5$ & $4.268 \mathrm{E}-6$ & $1.841 \mathrm{E}-2$ & $1.752 \mathrm{E}-6$ & $2.387 \mathrm{E}-4$ & 1.4 & 8.0 & $2.387 \mathrm{E}-5$ & $9 \mathrm{E}-8$ & BE-7 & 1E-3 \\
\hline 40 & $1.535 \mathrm{E}-5$ & $4.412 \mathrm{E}-6$ & $1.838 \mathrm{E}-2$ & $1.980 \mathrm{E}-6$ & $2.274 \mathrm{E}-4$ & $1.449 \mathrm{E}-4$ & 8.085E-5 & $2.616 \mathrm{E}-5$ & 4E-8 & $1.271 \mathrm{E}-7$ & BE-3 \\
\hline 42 & $1.443 \mathrm{E}-5$ & $4.546 \mathrm{E}-6$ & $1.835 \mathrm{E}-2$ & $2.220 \mathrm{E}-6$ & $2.170 \mathrm{E}-4$ & $1.441 \mathrm{E}-4$ & $8.124 E-5$ & $2.849 \mathrm{E}-5$ & $9.991 \mathrm{E}-9$ & 6E-7 & 4E-3 \\
\hline 44 & $1.355 \mathrm{E}-5$ & $4.672 \mathrm{E}-6$ & $1.832 \mathrm{E}-2$ & $2.470 \mathrm{E}-6$ & $2.074 \mathrm{E}-4$ & $1.429 E-4$ & $8.135 E-5$ & $3.087 \mathrm{E}-5$ & 9.752E-9 & $1.221 \mathrm{E}-7$ & $1.440 \mathrm{E}-3$ \\
\hline 46 & $1.269 \mathrm{E}-5$ & $4.788 \mathrm{E}-6$ & $1.828 \mathrm{E}-2$ & 2.728E-6 & $1.987 \mathrm{E}-4$ & $1.414 \mathrm{E}-4$ & $8.120 \mathrm{E}-5$ & $3.328 \mathrm{E}-5$ & $9.523 \mathrm{E}-9$ & $1.196 \mathrm{E}-7$ & $1.507 \mathrm{E}-3$ \\
\hline 48 & $1.187 \mathrm{E}-5$ & $4.894 \mathrm{E}-6$ & $1.825 \mathrm{E}-2$ & 2.994E-6 & $1.908 \mathrm{E}-4$ & $1.397 \mathrm{E}-4$ & $8.080 \mathrm{E}-5$ & $3.570 \mathrm{E}-5$ & $9.304 \mathrm{E}-9$ & $1.173 \mathrm{E}-7$ & $1.573 \mathrm{E}-3$ \\
\hline 50 & $1.109 \mathrm{E}-5$ & 4.992E-6 & 1.822E-2 & 3.264E-6 & 1.83 & $1.377 \mathrm{E}-4$ & 8.019E-5 & 3.813E-5 & $9.094 \mathrm{E}-9$ & $1.150 \mathrm{E}-7$ & $1.640 \mathrm{E}-3$ \\
\hline 52 & $1.033 \mathrm{E}-5$ & $5.080 \mathrm{E}-6$ & $1.818 \mathrm{E}-2$ & $3.53 \underset{8 \mathrm{E}-6}{ }$ & $1.772 \mathrm{E}-4$ & $1.356 \mathrm{E}-4$ & 7.939E-5 & $4.055 \mathrm{E}-5$ & 8.894E-9 & $1.128 \mathrm{E}-7$ & $1.706 \mathrm{E}-3$ \\
\hline 54 & $9.616 \mathrm{E}-6$ & $5.159 \mathrm{E}-6$ & $1.815 \mathrm{E}-2$ & 3.813E-6 & 1.713E-4 & $1.333 \mathrm{E}-4$ & $7.843 E-5$ & $4.296 \mathrm{E}-5$ & 8.703E-9 & $1.107 \mathrm{E}-7$ & $1.773 \mathrm{E}-3$ \\
\hline 56 & $8.934 \mathrm{E}-6$ & $5.230 \mathrm{E}-6$ & $1.812 \mathrm{E}-2$ & $4.087 \mathrm{E}-6$ & $1.660 \mathrm{E}-4$ & $1.308 \mathrm{E}-4$ & $7.733 E-5$ & $4.535 \mathrm{E}-5$ & 8.522E-9 & $1.087 \mathrm{E}-7$ & $1.839 \mathrm{E}-3$ \\
\hline 58 & $8.286 \mathrm{E}-6$ & $5.292 \mathrm{E}-6$ & $1.808 \mathrm{E}-2$ & $4.358 \mathrm{E}-6$ & $1.612 \mathrm{E}-4$ & $1.283 \mathrm{E}-4$ & 7.612 & $4.770 \mathrm{E}-5$ & $8.350 \mathrm{E}-9$ & $1.068 \mathrm{E}-7$ & $1.906 \mathrm{E}-3$ \\
\hline 60 & $7.672 \mathrm{E}-6$ & 5.345E-6 & $1.805 \mathrm{E}-2$ & 4.625E-6 & $1.569 \mathrm{E}-4$ & $1.257 \mathrm{E}-4$ & $7.483 \mathrm{E}-5$ & $5.000 \mathrm{E}-5$ & $8.187 \mathrm{E}-9$ & $1.049 \mathrm{E}-7$ & $1.972 \mathrm{E}-3$ \\
\hline
\end{tabular}


Table 4.15. Isotopic concentrations in pin 72 vs burnup for Variant 12, State 1

\begin{tabular}{|c|c|c|c|c|c|c|c|c|c|c|c|}
\hline \multirow{2}{*}{$\begin{array}{c}\text { Burnup } \\
\text { (MWd/kg) }\end{array}$} & \multicolumn{11}{|c|}{ Concentration (atoms $/ \mathrm{b} \cdot \mathrm{cm}$, in fuel) } \\
\hline & ${ }^{235} \mathrm{U}$ & ${ }^{236} \mathrm{U}$ & ${ }^{238} \mathrm{U}$ & ${ }^{238} \mathrm{Pu}$ & ${ }^{239} \mathrm{Pu}$ & ${ }^{240} \mathrm{Pu}$ & ${ }^{241} \mathrm{Pu}$ & ${ }^{242} \mathrm{Pu}$ & ${ }^{\mathrm{T} 35} \mathrm{Xe}$ & ${ }^{149} \mathrm{Sm}$ & Total FP \\
\hline 0 & $8.737 \mathrm{E}-4$ & 0.0 & $1.874 \mathrm{E}-2$ & $\overline{0.0}$ & 0.0 & 0.0 & 0.0 & 0.0 & $9.458 \mathrm{E}-9$ & $7.367 \mathrm{E}-8$ & $8.312 \mathrm{E}-8$ \\
\hline 2 & 8.259E-4 & 8.833E-6 & $1.872 \mathrm{E}-2$ & $1.318 \mathrm{E}-9$ & $1.646 \mathrm{E}-5$ & $5.108 \mathrm{E}-7$ & $4.201 \mathrm{E}-8$ & 4.307E-10 & $9.697 \mathrm{E}-9$ & $7.519 \mathrm{E}-8$ & $6.784 \mathrm{E}-5$ \\
\hline 4 & $7.808 \mathrm{E}-4$ & $1.714 \mathrm{E}-5$ & $1.870 \mathrm{E}-2$ & 8.317E-9 & $3.154 \mathrm{E}-5$ & $1.881 \mathrm{E}-6$ & $3.136 \mathrm{E}-7$ & $6.726 \mathrm{E}-9$ & 9.754E-9 & $7.969 \mathrm{E}-8$ & $1.355 \mathrm{E}-4$ \\
\hline 6 & $7.378 \mathrm{E}-4$ & $2.499 \mathrm{E}-5$ & $1.868 \mathrm{E}-2$ & $2.391 \mathrm{E}-8$ & $4.439 \mathrm{E}-5$ & $3.776 \mathrm{E}-6$ & $9.201 \mathrm{E}-7$ & $3.071 \mathrm{E}-8$ & $9.766 \mathrm{E}-9$ & $8.348 \mathrm{E}-8$ & $2.036 \mathrm{E}-4$ \\
\hline 8 & $6.969 \mathrm{E}-4$ & $3.242 \mathrm{E}-5$ & $1.865 \mathrm{E}-2$ & $5.059 \mathrm{E}-8$ & $5.540 \mathrm{E}-5$ & $5.989 \mathrm{E}-6$ & $1.867 \mathrm{E}-6$ & $8.594 \mathrm{E}-8$ & $9.744 \mathrm{E}-9$ & $8.663 \mathrm{E}-8$ & $2.717 \mathrm{E}-4$ \\
\hline 10 & $6.578 \mathrm{E}-4$ & 3.945E-5 & $1.863 \mathrm{E}-2$ & $9.064 \mathrm{E}-8$ & $6.486 \mathrm{E}-5$ & $8.399 \mathrm{E}-6$ & $3.114 \mathrm{E}-6$ & $1.849 \mathrm{E}-7$ & $9.697 \mathrm{E}-9$ & $8.923 \mathrm{E}-8$ & $3.399 \mathrm{E}-4$ \\
\hline 12 & $6.204 \mathrm{E}-4$ & $4.610 \mathrm{E}-5$ & $1.861 \mathrm{E}-2$ & $1.462 \mathrm{E}-7$ & 7.3 & E-5 & 4.604E-6 & $3.381 \mathrm{E}-7$ & $9.630 \mathrm{E}-9$ & $9.134 \mathrm{E}-8$ & $80 \mathrm{E}-4$ \\
\hline 14 & $5.847 \mathrm{E}-4$ & $5.240 \mathrm{E}-5$ & $1.858 \mathrm{E}-2$ & $2.192 \mathrm{E}-7$ & 8.00 & 1.2 & $6.281 \mathrm{E}-6$ & $5.534 \mathrm{E}-7$ & $9.547 \mathrm{E}-9$ & 3E-8 & 2E-4 \\
\hline 16 & $5.504 \mathrm{E}-4$ & $5.835 \mathrm{E}-5$ & $1.856 \mathrm{E}-2$ & $3.114 \mathrm{E}-7$ & $8.609 \mathrm{E}-5$ & 1.6 & $8.089 \mathrm{E}-6$ & 8.367E-7 & $9.452 \mathrm{E}-9$ & $4 \mathrm{E}-8$ & $3 \mathrm{E}-4$ \\
\hline 18 & $5.177 \mathrm{E}-4$ & $6.398 \mathrm{E}-5$ & $1.853 \mathrm{E}-2$ & 4.245E-7 & $9.129 \mathrm{E}-5$ & $1.878 \mathrm{E}-5$ & 9.982E-6 & $1.192 \mathrm{E}-6$ & 9.347E-9 & $9.531 \mathrm{E}-8$ & $24 E-4$ \\
\hline 20 & $4.863 \mathrm{E}-4$ & $6.928 \mathrm{E}-5$ & $1.851 \mathrm{E}-2$ & $5.601 \mathrm{E}-7$ & $9.573 \mathrm{E}-5$ & $2.139 \mathrm{E}-5$ & $1.192 \mathrm{E}-5$ & $1.621 \mathrm{E}-6$ & $9.232 \mathrm{E}-9$ & $9.599 \mathrm{E}-8$ & $6.805 \mathrm{E}-4$ \\
\hline 22 & $4.563 \mathrm{E}-4$ & $7.428 \mathrm{E}-5$ & $1.848 \mathrm{E}-2$ & 7.193E-7 & $9.953 \mathrm{E}-5$ & $2.397 \mathrm{E}-5$ & $1.387 \mathrm{E}-5$ & $2.125 \mathrm{E}-6$ & $9.110 \mathrm{E}-9$ & $9.639 \mathrm{E}-8$ & $7.485 \mathrm{E}-4$ \\
\hline 24 & $4.276 \mathrm{E}-4$ & 7.897E-5 & $1.845 \mathrm{E}-2$ & 9.035E-7 & 1.02 & $2.650 \mathrm{E}-5$ & $1.580 \mathrm{E}-5$ & $2.703 E-6$ & $8.982 \mathrm{E}-9$ & & $5 \mathrm{E}-4$ \\
\hline 26 & $4.002 \mathrm{E}-4$ & 8.337E-5 & $1.843 \mathrm{E}-2$ & $1.113 \mathrm{E}-6$ & 1.0 & 2.8 & 1.7 & 3.355E-6 & $50 \mathrm{E}-9$ & E-8 & E-4 \\
\hline 28 & $3.741 \mathrm{E}-4$ & $8.749 \mathrm{E}-5$ & $1.840 \mathrm{E}-2$ & $1.350 \mathrm{E}-6$ & -4 & -5 & -5 & .078E-6 & 13E-9 & E-8 & E-4 \\
\hline 30 & $3.491 \mathrm{E}-4$ & $9.133 \mathrm{E}-5$ & $1.837 \mathrm{E}-2$ & $1.613 \mathrm{E}-6$ & 1.09 & 3.3 & $2.130 \mathrm{E}-5$ & $4.869 \mathrm{E}-6$ & $8.573 \mathrm{E}-9$ & OE-8 & $20 \mathrm{E}-3$ \\
\hline 32 & $3.253 \mathrm{E}-4$ & 9.489E-5 & $1.834 \mathrm{E}-2$ & $1.903 \mathrm{E}-6$ & $1.112 \mathrm{E}-4$ & 3.593E-5 & 2.299E-5 & $5.725 \mathrm{E}-6$ & $8.432 \mathrm{E}-9$ & $9.538 \mathrm{E}-8$ & 38E-3 \\
\hline 34 & $3.027 \mathrm{E}-4$ & $9.819 \mathrm{E}-5$ & $1.831 \mathrm{E}-2$ & $2.221 \mathrm{E}-6$ & $1.124 \mathrm{E}-4$ & $3.809 \mathrm{E}-5$ & $2.459 \mathrm{E}-5$ & $6.643 \mathrm{E}-6$ & $8.289 \mathrm{E}-9$ & $9.473 \mathrm{E}-8$ & $1.156 \mathrm{E}-3$ \\
\hline 36 & $2.812 E-4$ & $1.012 \mathrm{E}-4$ & $1.828 \mathrm{E}-2$ & $2.565 \mathrm{E}-6$ & $1.134 \mathrm{E}-4$ & 4.0 & $2.609 \mathrm{E}-5$ & $7.619 \mathrm{E}-6$ & $8.146 \mathrm{E}-9$ & $9.398 \mathrm{E}-8$ & $1.224 \mathrm{E}-3$ \\
\hline 38 & $2.608 \mathrm{E}-4$ & $1.040 \mathrm{E}-4$ & $1.825 \mathrm{E}-2$ & $2.936 \mathrm{E}-6$ & $1.141 \mathrm{E}-4$ & 4.21 & $2.750 \mathrm{E}-5$ & $8.649 \mathrm{E}-6$ & $8.003 \mathrm{E}-9$ & $9.315 \mathrm{E}-8$ & $1.291 \mathrm{E}-3$ \\
\hline 40 & $2.414 \mathrm{E}-4$ & $1.066 \mathrm{E}-4$ & $1.822 \mathrm{E}-2$ & 2E-6 & & & -5 & 28E-6 & 1E-9 & $9.226 \mathrm{E}-8$ & $59 \mathrm{E}-3$ \\
\hline 42 & $2.231 \mathrm{E}-4$ & $1.089 \mathrm{E}-4$ & 1.8 & -6 & & -5 & -5 & 5E-5 & $7.721 \mathrm{E}-9$ & $9.130 \mathrm{E}-8$ & $1.427 \mathrm{E}-3$ \\
\hline 44 & 2.05 & $1.109 \mathrm{E}-4$ & $1.816 \mathrm{E}-2$ & 4.197E-6 & & & 3. & 32E-5 & HE-9 & $1 \mathrm{E}-8$ & $1.494 \mathrm{E}-3$ \\
\hline 46 & $1.896 \mathrm{E}-4$ & $1.128 \mathrm{E}-4$ & $1.813 \mathrm{E}-2$ & $4.663 \mathrm{E}-6$ & $1.154 \mathrm{E}-4$ & $4.906 \mathrm{E}-5$ & $3.214 \mathrm{E}-5$ & $1.322 \mathrm{E}-5$ & $7.449 \mathrm{E}-9$ & 8.9 & 2E-3 \\
\hline 48 & $1.743 \mathrm{E}-4$ & $1.144 \mathrm{E}-4$ & $1.810 \mathrm{E}-2$ & $5.148 \mathrm{E}-6$ & $1.154 \mathrm{E}-4$ & $5.055 \mathrm{E}-5$ & $3.306 \mathrm{E}-5$ & $1.445 \mathrm{E}-5$ & $7.318 \mathrm{E}-9$ & $8.827 \mathrm{E}-8$ & $1.629 \mathrm{E}-3$ \\
\hline 50 & $1.599 \mathrm{E}-4$ & $1.158 \mathrm{E}-4$ & $1.806 \mathrm{E}-2$ & 5.652 E-6 & $1.153 \mathrm{E}-4$ & $5.195 \mathrm{E}-5$ & 3.390E-5 & $1.571 \mathrm{E}-5$ & $7.190 \mathrm{E}-9$ & 8.724E-8 & $1.697 \mathrm{E}-3$ \\
\hline 52 & $1.465 \mathrm{E}-4$ & $1.170 \mathrm{E}-4$ & $1.803 E-2$ & 6.171E-6 & $1.151 \mathrm{E}-4$ & $5.325 \mathrm{E}-5$ & $3.465 \mathrm{E}-5$ & $1.700 \mathrm{E}-5$ & $7.068 \mathrm{E}-9$ & $8.621 \mathrm{E}-8$ & $1.764 \mathrm{E}-3$ \\
\hline 54 & $1.339 \mathrm{E}-4$ & $1.180 \mathrm{E}-4$ & $1.800 \mathrm{E}-2$ & $6.704 \mathrm{E}-6$ & $1.149 \mathrm{E}-4$ & $5.446 \mathrm{E}-5$ & $3.533 \mathrm{E}-5$ & $1.830 \mathrm{E}-5$ & $6.949 \mathrm{E}-9$ & $8.520 \mathrm{E}-8$ & $1.832 \mathrm{E}-3$ \\
\hline 56 & $1.222 \mathrm{E}-4$ & $1.188 \mathrm{E}-4$ & $1.796 \mathrm{E}-2$ & $7.248 \mathrm{E}-6$ & $1.147 \mathrm{E}-4$ & 5.559E-5 & 3.593E-5 & $1.961 \mathrm{E}-5$ & $6.837 \mathrm{E}-9$ & $8.422 \mathrm{E}-8$ & $1.899 \mathrm{E}-3$ \\
\hline 58 & $1.114 \mathrm{E}-4$ & $1.194 \mathrm{E}-4$ & $1.793 \mathrm{E}-2$ & $7.800 \mathrm{E}-6$ & $1.144 \mathrm{E}-4$ & $5.663 \mathrm{E}-5$ & $3.646 \mathrm{E}-5$ & $2.094 \mathrm{E}-5$ & $6.729 \mathrm{E}-9$ & 8.326E-8 & $1.967 \mathrm{E}-3$ \\
\hline 60 & $1.013 E-4$ & $1.199 \mathrm{E}-4$ & $1.789 \mathrm{E}-2$ & 8.359E-6 & $1.141 \mathrm{E}-4$ & $5.758 \mathrm{E}-5$ & 3.693E-5 & 2.227E-5 & $6.628 \mathrm{E}-9$ & 8.235E-8 & $4 \mathrm{E}-3$ \\
\hline
\end{tabular}


Table 4.16. Cell relative absorption reaction rates vs burnup for Variants 1-4 and 7-10, State 1

\begin{tabular}{|c|c|c|c|c|c|c|c|c|c|c|c|}
\hline \multirow{2}{*}{$\begin{array}{c}\text { Burnup } \\
\text { (MWd/kg) }\end{array}$} & \multicolumn{11}{|c|}{ Relative absorption rate } \\
\hline & ${ }^{235} \mathrm{U}$ & ${ }^{236} \mathrm{U}$ & ${ }^{238} \mathrm{U}$ & ${ }^{238} \mathrm{Pu}$ & ${ }^{239} \mathrm{Pu}$ & ${ }^{240} \mathrm{Pu}$ & ${ }^{241} \mathrm{Pu}$ & ${ }^{242} \mathrm{Pu}$ & ${ }^{135} \mathrm{Xe}$ & ${ }^{149} \mathrm{Sm}$ & Total FP \\
\hline \multicolumn{12}{|c|}{ Variant 1} \\
\hline 0 & $6.019 \mathrm{E}-1$ & 0.0 & $2.917 \mathrm{E}-1$ & 0.0 & 0.0 & 0.0 & 0.0 & 0.0 & $2.169 \mathrm{E}-2$ & $5.352 \mathrm{E}-3$ & $2.704 \mathrm{E}-2$ \\
\hline 10 & $4.118 \mathrm{E}-1$ & $4.703 \mathrm{E}-3$ & $2.851 \mathrm{E}-1$ & 4.112E-5 & $1.426 \mathrm{E}-1$ & $2.356 \mathrm{E}-2$ & $6.192 \mathrm{E}-3$ & $1.043 \mathrm{E}-4$ & $2.088 \mathrm{E}-2$ & $6.323 E-3$ & $5.238 \mathrm{E}-2$ \\
\hline 20 & $2.982 \mathrm{E}-1$ & 7.544E-3 & $2.823 \mathrm{E}-1$ & $2.442 \mathrm{E}-4$ & $1.986 \mathrm{E}-1$ & $4.499 \mathrm{E}-2$ & $2.205 \mathrm{E}-2$ & $8.569 \mathrm{E}-4$ & $1.962 \mathrm{E}-2$ & $6.742 \mathrm{E}-3$ & $7.268 \mathrm{E}-2$ \\
\hline 30 & $2.183 \mathrm{E}-1$ & 9.382E-3 & $2.807 \mathrm{E}-1$ & $6.937 \mathrm{E}-4$ & $2.253 \mathrm{E}-1$ & $5.902 \mathrm{E}-2$ & 3.875E-2 & $2.402 \mathrm{E}-3$ & $1.872 \mathrm{E}-2$ & $6.898 \mathrm{E}-3$ & $9.125 \mathrm{E}-2$ \\
\hline 40 & $1.580 \mathrm{E}-1$ & $1.058 \mathrm{E}-2$ & 2.794E-1 & $1.433 \mathrm{E}-3$ & $2.386 \mathrm{E}-1$ & $6.873 \mathrm{E}-2$ & $5.297 \mathrm{E}-2$ & $4.459 \mathrm{E}-3$ & $1.796 \mathrm{E}-2$ & $6.906 \mathrm{E}-3$ & $1.084 \mathrm{E}-1$ \\
\hline 50 & $1.120 \mathrm{E}-1$ & $1.126 \mathrm{E}-2$ & $2.780 \mathrm{E}-1$ & $2.448 \mathrm{E}-3$ & $2.449 \mathrm{E}-1$ & $7.541 \mathrm{E}-2$ & $6.385 \mathrm{E}-2$ & $6.690 \mathrm{E}-3$ & $1.729 \mathrm{E}-2$ & $6.830 \mathrm{E}-3$ & $1.239 \mathrm{E}-1$ \\
\hline 60 & $7.725 \mathrm{E}-2$ & $1.156 \mathrm{E}-2$ & 2.763E-1 & 3.666E-3 & $2.474 \mathrm{E}-1$ & $7.991 \mathrm{E}-2$ & $7.150 \mathrm{E}-2$ & $8.831 \mathrm{E}-3$ & $1.668 \mathrm{E}-2$ & $6.719 \mathrm{E}-3$ & $1.378 \mathrm{E}-1$ \\
\hline \multicolumn{12}{|c|}{ Variant 2} \\
\hline 0 & $1.462 \mathrm{E}-2$ & 0.0 & $2.694 \mathrm{E}-1$ & 0.0 & $5.852 \mathrm{E}-1$ & $6.365 \mathrm{E}-2$ & $6.401 \mathrm{E}-3$ & 0.0 & $8.911 \mathrm{E}-3$ & $2.220 \mathrm{E}-3$ & 1.113E-2 \\
\hline 10 & $1.238 \mathrm{E}-2$ & $1.939 \mathrm{E}-4$ & $2.687 \mathrm{E}-1$ & $1.828 \mathrm{E}-5$ & $4.864 \mathrm{E}-1$ & $9.587 \mathrm{E}-2$ & $3.547 \mathrm{E}-2$ & $1.173 \mathrm{E}-3$ & $1.563 \mathrm{E}-2$ & $5.664 \mathrm{E}-3$ & $4.823 \mathrm{E}-2$ \\
\hline 20 & $1.053 \mathrm{E}-2$ & $3.488 \mathrm{E}-4$ & $2.689 \mathrm{E}-1$ & $1.057 \mathrm{E}-4$ & $4.146 \mathrm{E}-1$ & $1.103 \mathrm{E}-1$ & $6.539 \mathrm{E}-2$ & 3.735E-3 & $1.564 \mathrm{E}-2$ & $6.088 \mathrm{E}-3$ & $7.067 \mathrm{E}-2$ \\
\hline 30 & $8.722 \mathrm{E}-3$ & $4.654 \mathrm{E}-4$ & $2.691 \mathrm{E}-1$ & $3.094 \mathrm{E}-4$ & $3.569 \mathrm{E}-1$ & $1.166 \mathrm{E}-1$ & $8.949 \mathrm{E}-2$ & $6.988 \mathrm{E}-3$ & $1.561 \mathrm{E}-2$ & $6.319 \mathrm{E}-3$ & $9.088 \mathrm{E}-2$ \\
\hline 40 & $7.007 \mathrm{E}-3$ & $5.503 E-4$ & $2.691 \mathrm{E}-1$ & $6.549 \mathrm{E}-4$ & $3.139 \mathrm{E}-1$ & $1.174 \mathrm{E}-1$ & 1.051E-1 & $1.025 \mathrm{E}-2$ & $1.552 \mathrm{E}-2$ & $6.431 \mathrm{E}-3$ & $1.094 \mathrm{E}-1$ \\
\hline 50 & $5.445 \mathrm{E}-3$ & $6.036 \mathrm{E}-4$ & $2.689 \mathrm{E}-1$ & $1.127 \mathrm{E}-3$ & $2.849 \mathrm{E}-1$ & $1.149 \mathrm{E}-1$ & $1.121 \mathrm{E}-1$ & $1.316 \mathrm{E}-2$ & $1.539 \mathrm{E}-2$ & $6.465 \mathrm{E}-3$ & $1.261 \mathrm{E}-1$ \\
\hline 60 & $4.094 \mathrm{E}-3$ & $6.300 \mathrm{E}-4$ & $2.683 \mathrm{E}-1$ & $1.671 \mathrm{E}-3$ & $2.667 \mathrm{E}-1$ & $1.105 \mathrm{E}-1$ & $1.127 \mathrm{E}-1$ & $1.553 \mathrm{E}-2$ & $1.524 \mathrm{E}-2$ & $6.459 \mathrm{E}-3$ & $1.410 \mathrm{E}-1$ \\
\hline \multicolumn{12}{|c|}{ Variant 3} \\
\hline 0 & $2.380 \mathrm{E}-1$ & $9.611 \mathrm{E}-3$ & $2.871 \mathrm{E}-1$ & $2.728 \mathrm{E}-3$ & $2.473 \mathrm{E}-1$ & $410 \mathrm{E}-2$ & $3.768 \mathrm{E}-2$ & $2.470 \mathrm{E}-3$ & $1.968 \mathrm{E}-2$ & 4.793E-3 & $2.447 \mathrm{E}-2$ \\
\hline \multicolumn{12}{|c|}{ Variant 4} \\
\hline 0 & $2.235 \mathrm{E}-1$ & $9.107 \mathrm{E}-3$ & $2.813 \mathrm{E}-1$ & $2.551 \mathrm{E}-3$ & $2.316 \mathrm{E}-1$ & $6.040 \mathrm{E}-2$ & $3.521 \mathrm{E}-2$ & $2.376 \mathrm{E}-3$ & $1.810 \mathrm{E}-2$ & $4.417 \mathrm{E}-3$ & $6.341 \mathrm{E}-2$ \\
\hline \multicolumn{12}{|c|}{ Variant 7} \\
\hline 0 & $1.590 \mathrm{E}-2$ & 0.0 & $2.735 \mathrm{E}-1$ & 0.0 & $6.448 \mathrm{E}-1$ & 0.0 & 0.0 & 0.0 & $1.004 \mathrm{E}-2$ & $2.501 \mathrm{E}-3$ & $1.254 \mathrm{E}-2$ \\
\hline \multicolumn{12}{|c|}{ Variant 8} \\
\hline 0 & $5.140 \mathrm{E}-1$ & 0.0 & $3.002 \mathrm{E}-1$ & 0.0 & 0.0 & $7.246 \mathrm{E}-2$ & 0.0 & 0.0 & $2.414 \mathrm{E}-2$ & $5.901 \mathrm{E}-3$ & $3.004 \mathrm{E}-2$ \\
\hline \multicolumn{12}{|c|}{ Variant 9} \\
\hline 0 & $1.552 \mathrm{E}-2$ & 0.0 & $2.652 \mathrm{E}-1$ & 0.0 & 0.0 & 0.0 & $6.554 \mathrm{E}-1$ & 0.0 & $9.255 \mathrm{E}-3$ & $2.336 \mathrm{E}-3$ & $1.159 \mathrm{E}-2$ \\
\hline \multicolumn{12}{|c|}{ Variant 10} \\
\hline 0 & $1.025 \mathrm{E}-2$ & 0.0 & $2.532 \mathrm{E}-1$ & $3.575 \mathrm{E}-3$ & $4.319 \mathrm{E}-1$ & $1.537 \mathrm{E}-1$ & $8.081 \mathrm{E}-2$ & $2.065 \mathrm{E}-2$ & $2.673 \mathrm{E}-3$ & $7.009 \mathrm{E}-4$ & $3.374 \mathrm{E}-3$ \\
\hline 10 & 8.907E-3 & $1.640 \mathrm{E}-4$ & $2.512 \mathrm{E}-1$ & $3.508 \mathrm{E}-3$ & $3.900 \mathrm{E}-1$ & $1.487 \mathrm{E}-1$ & $9.350 \mathrm{E}-2$ & $2.043 \mathrm{E}-2$ & $6.958 \mathrm{E}-3$ & $5.228 \mathrm{E}-3$ & $3.412 \mathrm{E}-2$ \\
\hline 20 & $7.836 \mathrm{E}-3$ & $2.910 \mathrm{E}-4$ & 2.497E-1 & $3.768 \mathrm{E}-3$ & 3.615E-1 & $1.449 \mathrm{E}-1$ & $1.036 \mathrm{E}-1$ & $2.059 \mathrm{E}-2$ & $7.052 \mathrm{E}-3$ & $5.538 \mathrm{E}-3$ & $5.255 \mathrm{E}-2$ \\
\hline 30 & $6.877 \mathrm{E}-3$ & $3.909 \mathrm{E}-4$ & $2.484 \mathrm{E}-1$ & $4.134 \mathrm{E}-3$ & $3.376 \mathrm{E}-1$ & $1.414 \mathrm{E}-1$ & $1.107 \mathrm{E}-1$ & $2.097 \mathrm{E}-2$ & $7.180 \mathrm{E}-3$ & $5.700 \mathrm{E}-3$ & $6.902 \mathrm{E}-2$ \\
\hline 40 & $6.011 \mathrm{E}-3$ & 4.690E-4 & $2.472 \mathrm{E}-1$ & $4.560 \mathrm{E}-3$ & $3.174 \mathrm{E}-1$ & $1.378 \mathrm{E}-1$ & $1.155 \mathrm{E}-1$ & $2.150 \mathrm{E}-2$ & $7.326 \mathrm{E}-3$ & 5.799E-3 & $8.422 \mathrm{E}-2$ \\
\hline 50 & $5.228 \mathrm{E}-3$ & $5.293 E-4$ & $2.460 \mathrm{E}-1$ & $5.025 \mathrm{E}-3$ & $3.001 \mathrm{E}-1$ & $1.342 \mathrm{E}-1$ & $1.183 \mathrm{E}-1$ & $2.208 \mathrm{E}-2$ & $7.481 \mathrm{E}-3$ & $5.863 \mathrm{E}-3$ & $9.848 \mathrm{E}-2$ \\
\hline 60 & $4.521 \mathrm{E}-3$ & $5.739 \mathrm{E}-4$ & $2.449 \mathrm{E}-1$ & $5.515 \mathrm{E}-3$ & $2.854 \mathrm{E}-1$ & $1.306 \mathrm{E}-1$ & $1.196 \mathrm{E}-1$ & $2.268 \mathrm{E}-2$ & $7.639 \mathrm{E}-3$ & $5.911 \mathrm{E}-3$ & $1.119 \mathrm{E}-1$ \\
\hline
\end{tabular}


Table 4.17. Cell relative absorption reaction rates for Variant 11, States 1 and 2

\begin{tabular}{|c|c|c|c|c|c|c|c|c|c|c|c|}
\hline \multirow{2}{*}{$\begin{array}{c}\text { Bumup } \\
\text { (MWd/kg) }\end{array}$} & \multicolumn{11}{|c|}{ Relative absorption rate } \\
\hline & ${ }^{135} \mathrm{U}$ & ${ }^{236} \mathrm{U}$ & ${ }^{238} \mathrm{U}$ & ${ }^{238} \mathrm{Pu}$ & ${ }^{239} \mathrm{Pu}$ & ${ }^{240} \mathrm{Pu}$ & ${ }^{241} \mathrm{Pu}$ & ${ }^{242} \mathrm{Pu}$ & ${ }^{135} \mathrm{Xe}$ & ${ }^{149} \mathrm{Sm}$ & Total FP \\
\hline \multicolumn{12}{|c|}{ Pin 1, State 1} \\
\hline 0 & $6.196 \mathrm{E}-1$ & 0.0 & $2.692 \mathrm{E}-1$ & 0.0 & 0.0 & 0.0 & 0.0 & 0.0 & $2.405 \mathrm{E}-2$ & $5.869 \mathrm{E}-3$ & $2.992 \mathrm{E}-2$ \\
\hline 10 & $4.293 \mathrm{E}-1$ & $4.335 \mathrm{E}-3$ & $2.643 \mathrm{E}-1$ & $4.110 \mathrm{E}-5$ & $1.409 \mathrm{E}-1$ & $2.261 \mathrm{E}-2$ & $6.384 \mathrm{E}-3$ & $9.806 \mathrm{E}-5$ & $2.278 \mathrm{E}-2$ & $489 \mathrm{E}-3$ & $5.482 \mathrm{E}-2$ \\
\hline 20 & $3.101 \mathrm{E}-1$ & $7.023 E-3$ & $2.635 \mathrm{E}-1$ & $2.539 \mathrm{E}-4$ & $1.968 \mathrm{E}-1$ & $4.451 \mathrm{E}-2$ & $2.351 E-2$ & $8.404 \mathrm{E}-4$ & $2.145 \mathrm{E}-2$ & $6.899 \mathrm{E}-3$ & $7.576 \mathrm{E}-2$ \\
\hline 30 & $2.234 \mathrm{E}-1$ & $8.809 \mathrm{E}-3$ & $2.643 \mathrm{E}-1$ & $7.482 \mathrm{E}-4$ & $2.229 \mathrm{E}-1$ & $5.927 \mathrm{E}-2$ & $4.183 \mathrm{E}-2$ & $2.424 \mathrm{E}-3$ & $2.038 \mathrm{E}-2$ & $7.042 \mathrm{E}-3$ & $9.530 \mathrm{E}-2$ \\
\hline 40 & $1.566 \mathrm{E}-1$ & $9.963 \mathrm{E}-3$ & $2.654 \mathrm{E}-1$ & $1.594 \mathrm{E}-3$ & $2.358 \mathrm{E}-1$ & $6.950 \mathrm{E}-2$ & $5.722 \mathrm{E}-2$ & $4.586 \mathrm{E}-3$ & $1.941 \mathrm{E}-2$ & $7.020 \mathrm{E}-3$ & $1.135 \mathrm{E}-1$ \\
\hline 50 & $1.052 \mathrm{E}-1$ & $1.061 \mathrm{E}-2$ & $2.660 \mathrm{E}-1$ & $2.785 \mathrm{E}-3$ & $2.419 \mathrm{E}-1$ & $7.647 \mathrm{E}-2$ & $6.852 \mathrm{E}-2$ & $6.959 \mathrm{E}-3$ & $1.849 \mathrm{E}-2$ & $6.899 \mathrm{E}-3$ & $1.301 \mathrm{E}-1$ \\
\hline 60 & $6.710 \mathrm{E}-2$ & $1.084 \mathrm{E}-2$ & $2.659 \mathrm{E}-1$ & $4.212 \mathrm{E}-3$ & 2.442E-1 & $8.090 \mathrm{E}-2$ & $7.583 \mathrm{E}-2$ & $9.250 \mathrm{E}-3$ & $1.767 \mathrm{E}-2$ & $6.732 \mathrm{E}-3$ & $1.447 \mathrm{E}-1$ \\
\hline \multicolumn{12}{|c|}{ Pin 1, State 2} \\
\hline 0 & $6.095 \mathrm{E}-1$ & 0.0 & $2.811 \mathrm{E}-1$ & 0.0 & 0.0 & 0.0 & 0.0 & 0.0 & $2.317 \mathrm{E}-2$ & 77E-3 & $.882 \mathrm{E}-2$ \\
\hline 10 & $4.227 \mathrm{E}-1$ & 7.479E-3 & 2.751E-1 & $4.034 \mathrm{E}-5$ & $1.381 \mathrm{E}-1$ & $2.279 \mathrm{E}-2$ & $6.249 \mathrm{E}-3$ & $1.000 \mathrm{E}-4$ & $2.198 \mathrm{E}-2$ & $6.263 \mathrm{E}-3$ & $5.388 \mathrm{E}-2$ \\
\hline 20 & $3.051 \mathrm{E}-1$ & $7.250 \mathrm{E}-3$ & $2.740 \mathrm{E}-1$ & $2.490 \mathrm{E}-4$ & $1.928 \mathrm{E}-1$ & $4.482 \mathrm{E}-2$ & $2.300 \mathrm{E}-2$ & $8.558 \mathrm{E}-4$ & $2.068 \mathrm{E}-2$ & $6.653 \mathrm{E}-3$ & $7.493 \mathrm{E}-2$ \\
\hline 30 & $2.195 \mathrm{E}-1$ & $9.097 \mathrm{E}-3$ & $2.746 \mathrm{E}-1$ & $7.326 \mathrm{E}-4$ & 2.182E-1 & $5.966 \mathrm{E}-2$ & $4.088 \mathrm{E}-2$ & $2.469 \mathrm{E}-3$ & $1.962 \mathrm{E}-2$ & $6.782 \mathrm{E}-3$ & $9.453 \mathrm{E}-2$ \\
\hline 40 & $1.536 \mathrm{E}-1$ & $1.029 \mathrm{E}-2$ & $2.757 \mathrm{E}-1$ & $1.558 \mathrm{E}-3$ & $2.306 \mathrm{E}-1$ & $6.995 \mathrm{E}-2$ & $5.584 \mathrm{E}-2$ & $4.674 \mathrm{E}-3$ & $1.864 \mathrm{E}-2$ & $6.747 \mathrm{E}-3$ & $1.128 \mathrm{E}-1$ \\
\hline 50 & $1.030 \mathrm{E}-1$ & $1.097 \mathrm{E}-2$ & $2.764 \mathrm{E}-1$ & $2.716 \mathrm{E}-3$ & $2.362 \mathrm{E}-1$ & $7.697 \mathrm{E}-2$ & $6.675 \mathrm{E}-2$ & $7.100 \mathrm{E}-3$ & $1.772 \mathrm{E}-2$ & $6.617 \mathrm{E}-3$ & $1.294 \mathrm{E}-1$ \\
\hline 60 & $6.559 \mathrm{E}-2$ & $1.122 \mathrm{E}-2$ & $2.763 \mathrm{E}-1$ & $4.100 \mathrm{E}-3$ & 2.381E-1 & $8.144 \mathrm{E}-2$ & $7.375 \mathrm{E}-2$ & $9.449 \mathrm{E}-3$ & $1.690 \mathrm{E}-2$ & $4 \mathrm{E}-3$ & $1.440 \mathrm{E}-1$ \\
\hline \multicolumn{12}{|c|}{ Pin 29, State 1} \\
\hline 0 & E-1 & 0.0 & $2.756 \mathrm{E}-1$ & 0.0 & 0.0 & 0.0 & 0.0 & 0.0 & $5 \mathrm{E}-2$ & 2E-3 & $2.872 \mathrm{E}-2$ \\
\hline 10 & $4.357 \mathrm{E}-1$ & $4.233 \mathrm{E}-3$ & 2.707E-1 & $3.567 \mathrm{E}-5$ & $1.341 \mathrm{E}-1$ & $2.077 \mathrm{E}-2$ & $5.352 \mathrm{E}-3$ & $7.997 \mathrm{E}-5$ & $2.179 \mathrm{E}-2$ & 4E-3 & $5.224 \mathrm{E}-2$ \\
\hline 20 & $3.227 \mathrm{E}-1$ & $6.877 \mathrm{E}-3$ & $2.688 \mathrm{E}-1$ & $2.165 \mathrm{E}-4$ & $1.904 \mathrm{E}-1$ & $4.123 \mathrm{E}-2$ & $2.016 \mathrm{E}-2$ & $6.970 \mathrm{E}-4$ & $2.072 \mathrm{E}-2$ & $6.816 \mathrm{E}-3$ & $7.208 \mathrm{E}-2$ \\
\hline 30 & 2.397E-1 & 8.657E-3 & $2.683 \mathrm{E}-1$ & $6.354 \mathrm{E}-4$ & $2.184 \mathrm{E}-1$ & $5.541 \mathrm{E}-2$ & $3.676 \mathrm{E}-2$ & $2.048 \mathrm{E}-3$ & $1.991 \mathrm{E}-2$ & $6.980 \mathrm{E}-3$ & $9.071 \mathrm{E}-2$ \\
\hline 40 & $1.745 \mathrm{E}-1$ & $9.847 \mathrm{E}-3$ & $2.682 \mathrm{E}-1$ & $1.362 \mathrm{E}-3$ & $2.329 \mathrm{E}-1$ & $6.565 \mathrm{E}-2$ & $5.161 \mathrm{E}-2$ & 3.951E-3 & $1.917 \mathrm{E}-2$ & $6.997 \mathrm{E}-3$ & $1.083 \mathrm{E}-1$ \\
\hline 50 & $1.226 \mathrm{E}-1$ & $1.057 \mathrm{E}-2$ & $2.682 \mathrm{E}-1$ & $2.413 \mathrm{E}-3$ & $2.402 \mathrm{E}-1$ & $7.308 \mathrm{E}-2$ & $6.338 \mathrm{E}-2$ & $6.114 \mathrm{E}-3$ & $1.844 \mathrm{E}-2$ & $6.918 \mathrm{E}-3$ & $1.247 \mathrm{E}-1$ \\
\hline 60 & $8.241 \mathrm{E}-2$ & $1.092 \mathrm{E}-2$ & $2.679 \mathrm{E}-1$ & $3.723 \mathrm{E}-3$ & $2.432 \mathrm{E}-1$ & $7.825 \mathrm{E}-2$ & $7.174 \mathrm{E}-2$ & $8.279 \mathrm{E}-3$ & $1.772 \mathrm{E}-2$ & $6.785 \mathrm{E}-3$ & $1.394 \mathrm{E}-1$ \\
\hline \multicolumn{12}{|c|}{ Pin 29, State 2} \\
\hline 0 & -1 & 0.0 & $248 \mathrm{E}-1$ & 0.0 & 0.0 & 0.0 & 0.0 & 0.0 & -2 & -3 & $2.597 \mathrm{E}-2$ \\
\hline 10 & $86 \mathrm{E}-1$ & $4.581 \mathrm{E}-3$ & $2.983 \mathrm{E}-1$ & $3.392 \mathrm{E}-5$ & $1.271 \mathrm{E}-1$ & $2.115 \mathrm{E}-2$ & $5.055 \mathrm{E}-3$ & $8.372 \mathrm{E}-5$ & $1.968 \mathrm{E}-2$ & $5.802 \mathrm{E}-3$ & $4.977 \mathrm{E}-2$ \\
\hline 20 & $3.095 \mathrm{E}-1$ & $7.441 \mathrm{E}-3$ & $2.958 \mathrm{E}-1$ & $2.054 \mathrm{E}-4$ & $1.802 \mathrm{E}-1$ & 4.197E-2 & $1.901 \mathrm{E}-2$ & $7.281 \mathrm{E}-4$ & $1.865 \mathrm{E}-2$ & $6.148 \mathrm{E}-3$ & $6.995 \mathrm{E}-2$ \\
\hline 30 & $2.293 \mathrm{E}-1$ & $9.379 \mathrm{E}-3$ & $2.950 \mathrm{E}-1$ & $6.009 \mathrm{E}-4$ & $2.062 \mathrm{E}-1$ & $5.638 \mathrm{E}-2$ & $3.458 \mathrm{E}-2$ & $2.140 \mathrm{E}-3$ & $1.787 \mathrm{E}-2$ & $6.277 \mathrm{E}-3$ & $8.883 \mathrm{E}-2$ \\
\hline 40 & 63E-1 & $1.068 \mathrm{E}-2$ & $2.948 \mathrm{E}-1$ & $1.283 \mathrm{E}-3$ & 2.1 & $6.6^{\prime}$ & $9 \mathrm{E}-2$ & & $5 \mathrm{E}-2$ & & $6 \mathrm{E}-1$ \\
\hline 50 & $1.165 \mathrm{E}-1$ & $1.149 \mathrm{E}-2$ & 2.947E-1 & $2.263 \mathrm{E}-3$ & $2.255 \mathrm{E}-1$ & $7.422 \mathrm{E}-2$ & $5.922 \mathrm{E}-2$ & $6.406 \mathrm{E}-3$ & $1.643 \mathrm{E}-2$ & $6.176 \mathrm{E}-3$ & $1.231 \mathrm{E}-1$ \\
\hline 60 & $7.800 \mathrm{E}-2$ & $1.187 \mathrm{E}-2$ & $2.943 E-1$ & $3.479 \mathrm{E}-3$ & $2.276 \mathrm{E}-1$ & $7.939 \mathrm{E}-2$ & $6.681 \mathrm{E}-2$ & $8.688 \mathrm{E}-3$ & $1.574 \mathrm{E}-2$ & $6.036 \mathrm{E}-3$ & $1.380 \mathrm{E}-1$ \\
\hline \multicolumn{12}{|c|}{ Pin 72 , State 1} \\
\hline 0 & 82E-1 & 0.0 & $2.712 \mathrm{E}-1$ & 0.0 & 0.0 & 0.0 & 0.0 & 0. & E-2 & $\mathrm{E}-3$ & $0 \mathrm{E}-2$ \\
\hline 10 & $4.327 \mathrm{E}-1$ & $4.291 \mathrm{E}-3$ & $2.663 \mathrm{E}-1$ & $3.882 \mathrm{E}-5$ & $1.380 \mathrm{E}-1$ & $2.178 \mathrm{E}-2$ & $5.940 \mathrm{E}-3$ & $9.023 \mathrm{E}-5$ & $4 \mathrm{E}-2$ & $8-3$ & $5.386 \mathrm{E}-2$ \\
\hline 20 & $3.160 \mathrm{E}-1$ & $6.957 \mathrm{E}-3$ & $2.651 \mathrm{E}-1$ & $2.384 \mathrm{E}-4$ & & $4.304 \mathrm{E}-2$ & 8E-2 & & E-2 & & 8E-2 \\
\hline 30 & $2.306 \mathrm{E}-1$ & $8.736 \mathrm{E}-3$ & $2.654 \mathrm{E}-1$ & $7.023 \mathrm{E}-4$ & $2.210 \mathrm{E}-1$ & $5.758 \mathrm{E}-2$ & $3.972 \mathrm{E}-2$ & $2.262 \mathrm{E}-3$ & $2.026 \mathrm{E}-2$ & $7.026 \mathrm{E}-3$ & 9.359E-2 \\
\hline 40 & $1.642 \mathrm{E}-1$ & $9.903 \mathrm{E}-3$ & $2.660 \mathrm{E}-1$ & $1.502 \mathrm{E}-3$ & $2.346 \mathrm{E}-1$ & $6.785 \mathrm{E}-2$ & $5.496 \mathrm{E}-2$ & 4.313E-3 & $1.938 \mathrm{E}-2$ & $7.019 \mathrm{E}-3$ & $1.116 \mathrm{E}-1$ \\
\hline 50 & 23E-1 & $1.058 \mathrm{E}-2$ & $2.665 \mathrm{E}-1$ & 2.642E-3 & $2.411 \mathrm{E}-1$ & & E-2 & & 3E-2 & & $1.282 \mathrm{E}-1$ \\
\hline 10 & $7.306 \mathrm{E}-2$ & $1.086 \mathrm{E}-2$ & $2.663 \mathrm{E}-1$ & $4.030 \mathrm{E}-3$ & $2.437 \mathrm{E}-1$ & $7.983 \mathrm{E}-2$ & $7.430 \mathrm{E}-2$ & $8.838 \mathrm{E}-3$ & $1.773 \mathrm{E}-2$ & $6.759 \mathrm{E}-3$ & $1.429 \mathrm{E}-1$ \\
\hline \multicolumn{12}{|c|}{ Pin 72, State 2} \\
\hline 0 & E-1 & 0.0 & $3.487 \mathrm{E}-1$ & 0. & & 0.0 & 0.0 & & & & E-2 \\
\hline 10 & 68E-1 & 281E-3 & $3.410 \mathrm{E}-1$ & $3.356 \mathrm{E}-5$ & $189 \mathrm{E}$ & $2.267 \mathrm{E}-2$ & 067E- & 032E-4 & $1.659 \mathrm{E}-2$ & $5 E-3$ & $4.725 \mathrm{E}-2$ \\
\hline 20 & $11 \mathrm{E}-1$ & $8.534 \mathrm{E}-3$ & $3.382 \mathrm{E}-1$ & $2.050 \mathrm{E}-4$ & $1.660 \mathrm{E}-1$ & $4.416 \mathrm{E}-2$ & $1.872 \mathrm{E}-2$ & $8.857 \mathrm{E}-4$ & $1.558 \mathrm{E}-2$ & $5.104 \mathrm{E}-3$ & $6.890 \mathrm{E}-2$ \\
\hline 30 & $2.038 \mathrm{E}-1$ & $1.071 \mathrm{E}-2$ & $3.375 \mathrm{E}-1$ & $5.992 \mathrm{E}-4$ & $1.878 \mathrm{E}-1$ & $5.858 \mathrm{E}-2$ & 5E-2 & & $1.478 \mathrm{E}-2$ & $5.184 \mathrm{E}-3$ & $8.900 \mathrm{E}-2$ \\
\hline 40 & $1.439 \mathrm{E}-1$ & $1.213 \mathrm{E}-2$ & $3.375 \mathrm{E}-1$ & $1.270 \mathrm{E}-3$ & $1.980 \mathrm{E}-1$ & $6.858 \mathrm{E}-2$ & $4.593 \mathrm{E}-2$ & $4.893 \mathrm{E}-3$ & $1.405 \mathrm{E}-2$ & $5.145 \mathrm{E}-3$ & $1.077 \mathrm{E}-1$ \\
\hline 50 & $9.760 \mathrm{E}-2$ & $1.297 \mathrm{E}-2$ & 3.373E-1 & $2.213 \mathrm{E}-3$ & $2.021 \mathrm{E}-1$ & $7.545 \mathrm{E}-2$ & $5.515 \mathrm{E}-2$ & $7.478 \mathrm{E}-3$ & $1.335 \mathrm{E}-2$ & $5.034 \mathrm{E}-3$ & $1.248 \mathrm{E}-1$ \\
\hline 60 & $6.301 \mathrm{E}-2$ & $1.330 \mathrm{E}-2$ & $3.363 \mathrm{E}-1$ & $3.348 \mathrm{E}-3$ & $2.029 \mathrm{E}-1$ & $7.987 \mathrm{E}-2$ & $6.117 \mathrm{E}-2$ & $1.001 \mathrm{E}-2$ & $1.270 \mathrm{E}-2$ & $4.892 \mathrm{E}-3$ & $1.399 \mathrm{E}-1$ \\
\hline
\end{tabular}


Table 4.18. Cell relative absorption reaction rates for Variant 12, States 1 and 2

\begin{tabular}{|c|c|c|c|c|c|c|c|c|c|c|c|}
\hline \multirow{2}{*}{$\begin{array}{c}\text { Burnup } \\
\text { (MWd/kg) }\end{array}$} & \multicolumn{11}{|c|}{ Relative absorption rate } \\
\hline & ${ }^{235} \mathrm{U}$ & ${ }^{236} \mathrm{U}$ & ${ }^{238} \mathrm{U}$ & ${ }^{238} \mathrm{Pu}$ & ${ }^{239} \mathrm{Pu}$ & ${ }^{40} \mathrm{Pu}$ & ${ }^{241} \mathrm{Pu}$ & ${ }^{242} \mathrm{Pu}$ & ${ }^{135} \mathrm{Xe}$ & ${ }^{149} \mathrm{Sm}$ & Total FP \\
\hline \multicolumn{12}{|c|}{ 1, State 1} \\
\hline 0 & $1.475 \mathrm{E}-2$ & 0.0 & $2.385 \mathrm{E}-1$ & 0.0 & $6.179 \mathrm{E}-1$ & $308 \mathrm{E}-2$ & $6.713 \mathrm{E}-3$ & 0.0 & $1.056 \mathrm{E}-2$ & $2.587 \mathrm{E}-3$ & $1.315 \mathrm{E}-2$ \\
\hline 10 & $1.281 \mathrm{E}-2$ & $1.513 \mathrm{E}-4$ & $2.421 \mathrm{E}-1$ & $1.836 \mathrm{E}-5$ & $5.032 \mathrm{E}-1$ & $9.663 \mathrm{E}-2$ & $3.915 \mathrm{E}-2$ & $1.099 \mathrm{E}-3$ & $1.819 \mathrm{E}-2$ & $5.855 \mathrm{E}-3$ & $5.158 \mathrm{E}-2$ \\
\hline 20 & $1.109 \mathrm{E}-2$ & $2.733 E-4$ & $2.471 \mathrm{E}-1$ & $1.147 \mathrm{E}-4$ & $4.152 \mathrm{E}-1$ & $1.143 \mathrm{E}-1$ & $7.462 \mathrm{E}-2$ & $3.457 \mathrm{E}-3$ & $1.795 \mathrm{E}-2$ & $6.256 \mathrm{E}-3$ & $7.469 \mathrm{E}-2$ \\
\hline 30 & 9.199E-3 & $3.692 \mathrm{E}-4$ & $2.518 \mathrm{E}-1$ & $3.562 \mathrm{E}-4$ & $3.445 \mathrm{E}-1$ & $1.218 \mathrm{E}-1$ & $1.029 \mathrm{E}-1$ & $6.398 \mathrm{E}-3$ & $1.764 \mathrm{E}-2$ & $6.472 \mathrm{E}-3$ & $9.630 \mathrm{E}-2$ \\
\hline 40 & $7.221 \mathrm{E}-3$ & $4.393 \mathrm{E}-4$ & $2.555 \mathrm{E}-1$ & $7.803 \mathrm{E}-4$ & $2.956 \mathrm{E}-1$ & $1.219 \mathrm{E}-1$ & $1.187 \mathrm{E}-1$ & $9.380 \mathrm{E}-3$ & $1.729 \mathrm{E}-2$ & $6.557 \mathrm{E}-3$ & $1.164 \mathrm{E}-1$ \\
\hline 50 & $5.336 \mathrm{E}-3$ & $4.840 \mathrm{E}-4$ & 2.581E-1 & $1.352 \mathrm{E}-3$ & $2.671 \mathrm{E}-1$ & $1.171 \mathrm{E}-1$ & $1.222 \mathrm{E}-1$ & $1.204 \mathrm{E}-2$ & $1.695 \mathrm{E}-2$ & $6.557 \mathrm{E}-3$ & $1.345 \mathrm{E}-1$ \\
\hline 60 & $3.715 \mathrm{E}-3$ & $5.057 \mathrm{E}-4$ & $2.595 \mathrm{E}-1$ & $1.970 \mathrm{E}-3$ & $2.528 \mathrm{E}-1$ & $1.103 \mathrm{E}-1$ & $1.177 \mathrm{E}-1$ & $1.421 \mathrm{E}-2$ & $1.664 \mathrm{E}-2$ & $6.512 \mathrm{E}-3$ & $1.502 \mathrm{E}-1$ \\
\hline \multicolumn{12}{|c|}{ Pin 1, State 2} \\
\hline 0 & $1.469 \mathrm{E}-2$ & 0.0 & $2.460 \mathrm{E}-1$ & 0.0 & $6.108 \mathrm{E}-1$ & $5.895 \mathrm{E}-2$ & $6.646 \mathrm{E}-3$ & 0.0 & $1.032 \mathrm{E}-2$ & $2.529 \mathrm{E}-3$ & $1.285 \mathrm{E}-2$ \\
\hline 10 & $1.274 \mathrm{E}-2$ & $1.539 \mathrm{E}-4$ & $2.495 \mathrm{E}-1$ & $1.823 \mathrm{E}-5$ & $4.971 \mathrm{E}-1$ & $9.629 \mathrm{E}-2$ & $3.871 \mathrm{E}-2$ & $1.104 \mathrm{E}-3$ & 1.775E-2 & $5.718 \mathrm{E}-3$ & $5.106 \mathrm{E}-2$ \\
\hline 20 & $1.100 \mathrm{E}-2$ & $2.785 \mathrm{E}-4$ & $2.548 \mathrm{E}-1$ & $1.135 \mathrm{E}-4$ & $4.094 \mathrm{E}-1$ & $1.140 \mathrm{E}-1$ & $7.360 \mathrm{E}-2$ & $3.479 \mathrm{E}-3$ & $1.747 \mathrm{E}-2$ & $6.093 \mathrm{E}-3$ & $7.420 \mathrm{E}-2$ \\
\hline 30 & $9.099 \mathrm{E}-3$ & $3.770 \mathrm{E}-4$ & $2.598 \mathrm{E}-1$ & $3.516 \mathrm{E}-4$ & $3.391 \mathrm{E}-1$ & $1.216 \mathrm{E}-1$ & $1.012 \mathrm{E}-1$ & $6.452 \mathrm{E}-3$ & $1.712 \mathrm{E}-2$ & $6.285 \mathrm{E}-3$ & $9.583 \mathrm{E}-2$ \\
\hline 40 & $7.124 \mathrm{E}-3$ & 4.495E-4 & $2.639 \mathrm{E}-1$ & $7.678 \mathrm{E}-4$ & $2.903 \mathrm{E}-1$ & $1.218 \mathrm{E}-1$ & $1.165 \mathrm{E}-1$ & $9.483 \mathrm{E}-3$ & $1.673 \mathrm{E}-2$ & $6.348 \mathrm{E}-3$ & $1.159 \mathrm{E}-1$ \\
\hline 50 & $5.252 \mathrm{E}-3$ & $4.962 \mathrm{E}-4$ & $2.668 \mathrm{E}-1$ & $1.327 \mathrm{E}-3$ & $2.618 \mathrm{E}-1$ & $1.172 \mathrm{E}-1$ & $1.197 \mathrm{E}-1$ & $1.220 \mathrm{E}-2$ & $1.635 \mathrm{E}-2$ & $6.329 \mathrm{E}-3$ & $1.340 \mathrm{E}-1$ \\
\hline 60 & 3.647E-3 & $5.195 \mathrm{E}-4$ & $2.685 \mathrm{E}-1$ & $1.928 \mathrm{E}-3$ & $2.474 \mathrm{E}-1$ & $1.105 \mathrm{E}-1$ & $1.150 \mathrm{E}-1$ & $1.443 \mathrm{E}-2$ & $1.600 \mathrm{E}-2$ & $6.267 E-3$ & 1.497E-1 \\
\hline \multicolumn{12}{|c|}{ Pin 29, State 1} \\
\hline 0 & $1.485 \mathrm{E}-2$ & 0.0 & $2.561 \mathrm{E}-1$ & 0.0 & & $04 \mathrm{E}-2$ & $6.586 \mathrm{E}-3$ & 0.0 & $772 E-3$ & $2.420 \mathrm{E}-3$ & $1.219 \mathrm{E}-2$ \\
\hline 10 & $1.291 \mathrm{E}-2$ & $1.519 \mathrm{E}-4$ & $2.555 \mathrm{E}-1$ & 1.609E-5 & $5.041 \mathrm{E}-1$ & $9.203 \mathrm{E}-2$ & 3.363E-2 & $9.459 \mathrm{E}-4$ & $1.644 \mathrm{E}-2$ & $5.740 \mathrm{E}-3$ & $4.757 \mathrm{E}-2$ \\
\hline 20 & $1.127 \mathrm{E}-2$ & $2.719 \mathrm{E}-4$ & $2.557 \mathrm{E}-1$ & $9.648 \mathrm{E}-5$ & $4.314 \mathrm{E}-1$ & $1.077 \mathrm{E}-1$ & $6.361 \mathrm{E}-2$ & $2.927 \mathrm{E}-3$ & $1.672 \mathrm{E}-2$ & $6.142 \mathrm{E}-3$ & $6.930 \mathrm{E}-2$ \\
\hline 30 & $9.576 \mathrm{E}-3$ & $3.658 \mathrm{E}-4$ & $2.564 \mathrm{E}-1$ & $2.955 \mathrm{E}-4$ & $3.685 \mathrm{E}-1$ & $1.160 \mathrm{E}-1$ & $9.005 \mathrm{E}-2$ & $5.448 \mathrm{E}-3$ & $1.688 \mathrm{E}-2$ & $6.385 \mathrm{E}-3$ & $8.963 \mathrm{E}-2$ \\
\hline 40 & $7.823 \mathrm{E}-3$ & $4.365 \mathrm{E}-4$ & $2.575 \mathrm{E}-1$ & $6.555 \mathrm{E}-4$ & $3.185 \mathrm{E}-1$ & $1.188 \mathrm{E}-1$ & $1.087 \mathrm{E}-1$ & $8.117 \mathrm{E}-3$ & $1.688 \mathrm{E}-2$ & $6.510 \mathrm{E}-3$ & $1.089 \mathrm{E}-1$ \\
\hline 50 & $6.088 \mathrm{E}-3$ & $4.855 \mathrm{E}-4$ & $2.587 \mathrm{E}-1$ & $1.173 \mathrm{E}-3$ & $2.834 \mathrm{E}-1$ & $1.173 \mathrm{E}-1$ & $1.177 \mathrm{E}-1$ & $1.065 \mathrm{E}-2$ & $1.673 \mathrm{E}-2$ & $6.550 \mathrm{E}-3$ & $1.269 \mathrm{E}-1$ \\
\hline 60 & $4.495 \mathrm{E}-3$ & $5.139 \mathrm{E}-4$ & $2.596 \mathrm{E}-1$ & $1.784 \mathrm{E}-3$ & $2.619 \mathrm{E}-1$ & $1.130 \mathrm{E}-1$ & $1.183 \mathrm{E}-1$ & $1.287 \mathrm{E}-2$ & $1.652 \mathrm{E}-2$ & $6.535 \mathrm{E}-3$ & $1.430 \mathrm{E}-1$ \\
\hline \multicolumn{12}{|c|}{ Pin 29, State 2} \\
\hline 0 & $1.467 \mathrm{E}-2$ & 0.0 & $2.783 \mathrm{E}-1$ & 0.0 & $5.772 \mathrm{E}-1$ & $6.110 \mathrm{E}-2$ & $6.380 \mathrm{E}-3$ & 0.0 & $8.959 \mathrm{E}-3$ & $2.225 \mathrm{E}-3$ & $1.118 \mathrm{E}-2$ \\
\hline 10 & $1.270 \mathrm{E}-2$ & $1.599 \mathrm{E}-4$ & $2.777 \mathrm{E}-1$ & $1.570 \mathrm{E}-5$ & $4.848 \mathrm{E}-1$ & $9.209 \mathrm{E}-2$ & $3.244 \mathrm{E}-2$ & $9.633 \mathrm{E}-4$ & $1.502 \mathrm{E}-2$ & $5.259 \mathrm{E}-3$ & $4.592 \mathrm{E}-2$ \\
\hline 20 & $1.102 \mathrm{E}-2$ & $2.876 \mathrm{E}-4$ & $2.783 \mathrm{E}-1$ & $9.344 \mathrm{E}-5$ & $4.130 \mathrm{E}-1$ & 1.079E-1 & $6.097 \mathrm{E}-2$ & $2.995 \mathrm{E}-3$ & $1.520 \mathrm{E}-2$ & $5.596 \mathrm{E}-3$ & $6.791 \mathrm{E}-2$ \\
\hline 30 & $9.303 \mathrm{E}-3$ & $3.888 E-4$ & $2.796 \mathrm{E}-1$ & $2.841 \mathrm{E}-4$ & $3.512 \mathrm{E}-1$ & $1.163 \mathrm{E}-1$ & $8.579 \mathrm{E}-2$ & $5.602 \mathrm{E}-3$ & $1.527 \mathrm{E}-2$ & $5.788 \mathrm{E}-3$ & $8.847 \mathrm{E}-2$ \\
\hline 40 & $7.552 \mathrm{E}-3$ & $4.661 \mathrm{E}-4$ & $2.812 \mathrm{E}-1$ & $6.257 \mathrm{E}-4$ & $3.021 \mathrm{E}-1$ & $1.192 \mathrm{E}-1$ & $1.030 \mathrm{E}-1$ & $8.389 \mathrm{E}-3$ & $1.519 \mathrm{E}-2$ & $5.871 \mathrm{E}-3$ & $1.080 \mathrm{E}-1$ \\
\hline 50 & $5.843 \mathrm{E}-3$ & $5.205 \mathrm{E}-4$ & $2.828 \mathrm{E}-1$ & $1.113 \mathrm{E}-3$ & $2.676 \mathrm{E}-1$ & $1.177 \mathrm{E}-1$ & $1.109 \mathrm{E}-1$ & $1.106 \mathrm{E}-2$ & $1.499 \mathrm{E}-2$ & $5.878 \mathrm{E}-3$ & $1.260 \mathrm{E}-1$ \\
\hline 4 & $4.292 \mathrm{E}-3$ & $5.526 \mathrm{E}-4$ & $2.840 \mathrm{E}-1$ & $1.682 \mathrm{E}-3$ & $2.464 \mathrm{E}-1$ & $1.134 \mathrm{E}-1$ & $1.110 \mathrm{E}-1$ & $1.340 \mathrm{E}-2$ & $1.473 \mathrm{E}-2$ & $5.842 \mathrm{E}-3$ & $1.422 \mathrm{E}-1$ \\
\hline \multicolumn{12}{|c|}{ Pin 72, State 1} \\
\hline 0 & $1.481 \mathrm{E}-2$ & 0.0 & $2.455 \mathrm{E}-1$ & 0.0 & $6.102 \mathrm{E}-1$ & $5.988 \mathrm{E}-2$ & $6.667 \mathrm{E}-3$ & 0.0 & $1.026 \mathrm{E}-2$ & $2.526 \mathrm{E}-3$ & $1.279 \mathrm{E}-2$ \\
\hline 10 & $1.287 \mathrm{E}-2$ & $1.514 \mathrm{E}-4$ & $2.471 \mathrm{E}-1$ & $1.734 \mathrm{E}-5$ & EE-1 & $9.450 \mathrm{E}-2$ & $3.670 \mathrm{E}-2$ & $1.030 \mathrm{E}-3$ & $1.752 \mathrm{E}-2$ & $3 E-3$ & $4.995 \mathrm{E}-2$ \\
\hline 20 & $1.119 \mathrm{E}-2$ & $2.722 \mathrm{E}-4$ & $2.499 \mathrm{E}-1$ & $1.066 \mathrm{E}-4$ & $4.231 \mathrm{E}-1$ & $1.114 \mathrm{E}-1$ & $6.989 \mathrm{E}-2$ & $3.217 \mathrm{E}-3$ & $1.753 \mathrm{E}-2$ & $6.216 \mathrm{E}-3$ & $7.251 \mathrm{E}-2$ \\
\hline 30 & $9.394 \mathrm{E}-3$ & $3.671 \mathrm{E}-4$ & $2.529 \mathrm{E}-1$ & $3.303 \mathrm{E}-4$ & $3.550 \mathrm{E}-1$ & $1.195 \mathrm{E}-1$ & $9.771 \mathrm{E}-2$ & $5.973 \mathrm{E}-3$ & $1.743 \mathrm{E}-2$ & $6.446 \mathrm{E}-3$ & $9.364 \mathrm{E}-2$ \\
\hline 40 & $7.507 \mathrm{E}-3$ & $4.375 \mathrm{E}-4$ & $2.556 \mathrm{E}-1$ & $7.299 \mathrm{E}-4$ & $3.047 \mathrm{E}-1$ & $1.209 \mathrm{E}-1$ & $1.151 \mathrm{E}-1$ & $8.827 \mathrm{E}-3$ & $1.721 \mathrm{E}-2$ & $6.547 \mathrm{E}-3$ & $1.135 \mathrm{E}-1$ \\
\hline 50 & $5.668 \mathrm{E}-3$ & $4.842 \mathrm{E}-4$ & $2.577 \mathrm{E}-1$ & $1.285 \mathrm{E}-3$ & $2.728 \mathrm{E}-1$ & $1.175 \mathrm{E}-1$ & $1.211 \mathrm{E}-1$ & $1.145 \mathrm{E}-2$ & $1.694 \mathrm{E}-2$ & $6.562 \mathrm{E}-3$ & $1.316 \mathrm{E}-1$ \\
\hline 60 & $4.037 \mathrm{E}-3$ & $5.087 \mathrm{E}-4$ & $2.590 \mathrm{E}-1$ & $1.908 \mathrm{E}-3$ & $2.555 \mathrm{E}-1$ & $.117 \mathrm{E}-1$ & $1.186 \mathrm{E}-1$ & $1.365 \mathrm{E}-2$ & $1.664 \mathrm{E}-2$ & $6.527 \mathrm{E}-3$ & $1.476 \mathrm{E}-1$ \\
\hline \multicolumn{12}{|c|}{ Pin 72, State 2} \\
\hline 0 & $1.438 \mathrm{E}-2$ & 0.0 & 3.14 & 0.6 & $5.437 \mathrm{E}-1$ & $6.045 \mathrm{E}-2$ & $6.056 \mathrm{E}-3$ & 0.0 & 7.713E-3 & E-3 & $9.643 \mathrm{E}-3$ \\
\hline 10 & $1.230 \mathrm{E}-2$ & $1.810 \mathrm{E}-4$ & $3.151 \mathrm{E}-1$ & $1.606 \mathrm{E}-5$ & $4.450 \mathrm{E}-1$ & $9.433 \mathrm{E}-2$ & $3.282 \mathrm{E}-2$ & $1.139 \mathrm{E}-3$ & $1.306 \mathrm{E}-2$ & $4.397 \mathrm{E}-3$ & $4.552 \mathrm{E}-2$ \\
\hline 20 & $1.048 \mathrm{E}-2$ & $3.262 \mathrm{E}-4$ & $3.178 \mathrm{E}-1$ & $9.659 \mathrm{E}-5$ & $3.685 \mathrm{E}-1$ & $1.105 \mathrm{E}-1$ & $6.137 \mathrm{E}-2$ & $3.567 \mathrm{E}-3$ & $1.294 \mathrm{E}-2$ & $4.653 \mathrm{E}-3$ & $6.937 \mathrm{E}-2$ \\
\hline 30 & 8.632E-3 & $4.414 \mathrm{E}-4$ & $3.207 \mathrm{E}-1$ & $2.932 \mathrm{E}-4$ & $3.057 \mathrm{E}-1$ & $1.178 \mathrm{E}-1$ & $8.440 \mathrm{E}-2$ & $6.644 \mathrm{E}-3$ & $1.277 \mathrm{E}-2$ & 4.781E-3 & $9.143 \mathrm{E}-2$ \\
\hline 40 & $6.779 \mathrm{E}-3$ & $5.278 \mathrm{E}-4$ & $3.234 \mathrm{E}-1$ & $6.359 \mathrm{E}-4$ & $2.597 \mathrm{E}-1$ & $1.188 \mathrm{E}-1$ & $9.799 \mathrm{E}-2$ & $9.851 \mathrm{E}-3$ & $1.251 \mathrm{E}-2$ & $4.814 \mathrm{E}-3$ & $1.119 \mathrm{E}-1$ \\
\hline 50 & $5.042 \mathrm{E}-3$ & $5.857 \mathrm{E}-4$ & $3.254 \mathrm{E}-1$ & $1.102 \mathrm{E}-3$ & $2.305 \mathrm{E}-1$ & 1.152E-1 & $1.019 \mathrm{E}-1$ & $1.281 \mathrm{E}-2$ & $1.222 \mathrm{E}-2$ & $4.791 \mathrm{E}-3$ & $1.304 \mathrm{E}-1$ \\
\hline 60 & $3.545 \mathrm{E}-3$ & $6.168 \mathrm{E}-4$ & $3.265 \mathrm{E}-1$ & $1.614 \mathrm{E}-3$ & $2.142 \mathrm{E}-1$ & $1.094 \mathrm{E}-1$ & $9.871 \mathrm{E}-2$ & $1.530 \mathrm{E}-2$ & $1.194 \mathrm{E}-2$ & 4.733E-3 & $1.466 \mathrm{E}-1$ \\
\hline
\end{tabular}


Table 4.19. Fuel-averaged microscopic absorption cross section for Variant 11, States 1 and 2

\begin{tabular}{|c|c|c|c|c|c|c|c|c|c|c|c|}
\hline \multirow{2}{*}{$\begin{array}{c}\text { Bumup } \\
(\mathrm{MWd} / \mathrm{kg})\end{array}$} & \multicolumn{11}{|c|}{ Microscopic absorption cross section (b) } \\
\hline & ${ }^{235} \mathrm{U}$ & ${ }^{236} \mathrm{U}$ & ${ }^{238} \mathrm{U}$ & ${ }^{238} \mathrm{Pu}$ & ${ }^{239} \mathrm{Pu}$ & ${ }^{240} \mathrm{Pu}$ & ${ }^{241} \mathrm{Pu}$ & ${ }^{242} \mathrm{Pu}$ & ${ }^{133} \mathrm{Xe}$ & ${ }^{149} \mathrm{Sm}$ & Total FP \\
\hline \multicolumn{12}{|c|}{ Pin 1, State 1} \\
\hline 0 & $5.068 \mathrm{E}+1$ & $8.416 \mathrm{E}+0$ & $1.026 \mathrm{E}+0$ & $3.669 \mathrm{E}+1$ & $1.863 \mathrm{E}+2$ & $2.409 E+2$ & $1.642 \mathrm{E}+2$ & $3.119 \mathrm{E}+1$ & $1.817 \mathrm{E}+5$ & $5.694 \mathrm{E}+3$ & $2.572 \mathrm{E}+4$ \\
\hline 10 & $4.782 \mathrm{E}+1$ & $7.728 \mathrm{E}+0$ & $1.027 \mathrm{E}+0$ & $3.126 \mathrm{E}+1$ & $1.543 \mathrm{E}+2$ & $1.848 E+2$ & $1.387 \mathrm{E}+2$ & $3.433 E+1$ & $1.704 \mathrm{E}+5$ & $5.314 E+3$ & $1.125 \mathrm{E}+1$ \\
\hline 20 & $4.738 E+1$ & $7.174 E+0$ & $1.032 E+0$ & $3.115 \mathrm{E}+1$ & $1.478 E+2$ & $1.449 E+2$ & $1.352 \mathrm{E}+2$ & $3.384 \mathrm{E}+1$ & $1.697 \mathrm{E}+5$ & $5.265 E+3$ & $7.782 E+0$ \\
\hline 30 & $4.804 \mathrm{E}+1$ & $6.838 \mathrm{E}+0$ & $1.038 \mathrm{E}+0$ & $3.176 \mathrm{E}+1$ & $1.468 \mathrm{E}+2$ & $1.238 \mathrm{E}+2$ & $1.358 \mathrm{E}+2$ & $3.267 \mathrm{E}+1$ & $1.738 \mathrm{E}+5$ & $5.368 E+3$ & $6.515 \mathrm{E}+0$ \\
\hline 40 & $4.918 E+1$ & $6.630 \mathrm{E}+0$ & $1.045 \mathrm{E}+0$ & $3.268 \mathrm{E}+1$ & $1.482 E+2$ & $1.119 E+2$ & $1.383 \mathrm{E}+2$ & $3.107 \mathrm{E}+1$ & $1.800 E+5$ & $5.538 \mathrm{E}+3$ & $5.805 \mathrm{E}+0$ \\
\hline 50 & $5.044 \mathrm{E}+1$ & $6.504 \mathrm{E}+0$ & $1.051 \mathrm{E}+0$ & $3.367 \mathrm{E}+1$ & $1.506 \mathrm{E}+2$ & $1.049 E+2$ & $1.413 \mathrm{E}+2$ & $2.933 \mathrm{E}+1$ & $1.868 \mathrm{E}+5$ & $5.726 \mathrm{E}+3$ & $5.310 \mathrm{E}+0$ \\
\hline 60 & $5.155 \mathrm{E}+1$ & $6.434 \mathrm{E}+0$ & $1.057 \mathrm{E}+0$ & $3.453 \mathrm{E}+1$ & $1.530 \mathrm{E}+2$ & $1.006 \mathrm{E}+2$ & $1.441 \mathrm{E}+2$ & $2.769 E+1$ & $1.926 \mathrm{E}+5$ & $5.893 \mathrm{E}+3$ & $4.916 \mathrm{E}+0$ \\
\hline \multicolumn{12}{|c|}{ Pin 1, State 2} \\
\hline 0 & 4.672E+1 & $8.179 E+0$ & $1.004 \mathrm{E}+0$ & $3.367 \mathrm{E}+1$ & $1.710 \mathrm{E}+2$ & $2.283 E+2$ & $1.505 \mathrm{E}+2$ & $995 \mathrm{E}+1$ & $1.640 \mathrm{E}+5$ & $5.143 E+3$ & $2.322 \mathrm{E}+4$ \\
\hline 10 & $4.428 \mathrm{E}+1$ & $7.510 \mathrm{E}+0$ & $1.006 \mathrm{E}+0$ & $2.885 E+1$ & $1.422 \mathrm{E}+2$ & $1.752 E+2$ & $1.277 \mathrm{E}+2$ & $3.293 \mathrm{E}+1$ & $1.546 \mathrm{E}+5$ & $4.824 \mathrm{E}+3$ & $1.040 \mathrm{E}+1$ \\
\hline 20 & $4.390 \mathrm{E}+1$ & $6.975 E+0$ & $1.011 E+0$ & $2.877 \mathrm{E}+1$ & $1.364 \mathrm{E}+2$ & $1.375 E+2$ & $1.246 \mathrm{E}+2$ & $3.246 \mathrm{E}+1$ & $1.540 \mathrm{E}+5$ & $4.783 E+3$ & $7.249 \mathrm{E}+0$ \\
\hline 30 & 4.447E+1 & $6.653 \mathrm{E}+0$ & $1.016 \mathrm{E}+0$ & $2.930 \mathrm{E}+1$ & $1.353 \mathrm{E}+2$ & $1.174 \mathrm{E}+2$ & $1.251 \mathrm{E}+2$ & $3.135 \mathrm{E}+1$ & $1.576 E+5$ & $4.870 \mathrm{E}+3$ & $6.088 \mathrm{E}+0$ \\
\hline 40 & $4.544 E+1$ & $6.452 E+0$ & $1.022 \mathrm{E}+0$ & $3.009 \mathrm{E}+1$ & $1.365 \mathrm{E}+2$ & $1.060 E+2$ & $1.271 \mathrm{E}+2$ & $2.982 \mathrm{E}+1$ & $1.629 \mathrm{E}+5$ & $5.014 E+3$ & $5.432 \mathrm{E}+0$ \\
\hline 50 & $4.650 \mathrm{E}+1$ & $6.331 \mathrm{E}+0$ & $1.028 \mathrm{E}+0$ & $3.092 E+1$ & $1.385 E+2$ & $9.936 \mathrm{E}+1$ & $1.296 \mathrm{E}+2$ & $2.817 \mathrm{E}+1$ & $1.685 \mathrm{E}+5$ & $5.171 \mathrm{E}+3$ & $4.971 E+0$ \\
\hline 60 & $4.741 \mathrm{E}+1$ & $6.266 \mathrm{E}+0$ & $1.033 \mathrm{E}+0$ & $3.162 E+1$ & $1.403 E+2$ & $9.528 \mathrm{E}+1$ & $1.319 \mathrm{E}+2$ & $2.661 E+1$ & $1.734 E+5$ & $5.306 \mathrm{E}+3$ & $4.602 \mathrm{E}+0$ \\
\hline \multicolumn{12}{|c|}{ Pin 29, State 1} \\
\hline 0 & $4.612 \mathrm{E}+1$ & $8.204 E+0$ & 9.641E-1 & $3.296 E+1$ & $1.721 \mathrm{E}+2$ & $2.342 E+2$ & $E+2$ & $55 E+1$ & $1.598 E+5$ & $49 E+3$ & $2.266 E+4$ \\
\hline 10 & $4.318 E+1$ & $7.447 \mathrm{E}+0$ & 9.639E-1 & $2.794 \mathrm{E}+1$ & $1.403 E+2$ & $1.774 \mathrm{E}+2$ & $1.251 \mathrm{E}+2$ & $3.375 E+1$ & $1.484 \mathrm{E}+5$ & $4.669 \mathrm{E}+3$ & $1.081 \mathrm{E}+1$ \\
\hline 20 & $4.257 \mathrm{E}+1$ & $6.860 \mathrm{E}+0$ & $9.673 \mathrm{E}-1$ & 2.772E +1 & $1.330 \mathrm{E}+2$ & $1.363 \mathrm{E}+2$ & $1.211 \mathrm{E}+2$ & $3.329 \mathrm{E}+1$ & $1.471 E+5$ & $4.603 E+3$ & $7.457 \mathrm{E}+0$ \\
\hline 30 & 4.305E+1 & $6.496 \mathrm{E}+0$ & $9.723 \mathrm{E}-1$ & $2.821 \mathrm{E}+1$ & $1.313 E+2$ & $1.142 E+2$ & $1.211 \mathrm{E}+2$ & $3.216 \mathrm{E}+1$ & $1.504 \mathrm{E}+5$ & $4.685 E+3$ & $6.236 \mathrm{E}+0$ \\
\hline 40 & $4.405 \mathrm{E}+1$ & $6.262 E+0$ & $9.777 \mathrm{E}-1$ & $2.903 \mathrm{E}+1$ & $1.323 \mathrm{E}+2$ & $1.016 \mathrm{E}+2$ & $1.231 \mathrm{E}+2$ & $3.058 \mathrm{E}+1$ & $1.561 \mathrm{E}+5$ & $4.840 \mathrm{E}+3$ & $5.559 E+0$ \\
\hline 50 & $4.525 \mathrm{E}+1$ & $6.112 \mathrm{E}+0$ & $9.834 \mathrm{E}-1$ & $2.996 \mathrm{E}+1$ & $1.344 \mathrm{E}+2$ & $9.401 \mathrm{E}+1$ & $1.260 \mathrm{E}+2$ & $2.882 E+1$ & $1.625 \mathrm{E}+5$ & $5.021 E+3$ & $5.093 E+0$ \\
\hline 60 & $4.636 \mathrm{E}+1$ & $6.022 E+0$ & $9.886 \mathrm{E}-1$ & $3.083 \mathrm{E}+1$ & $1.368 \mathrm{E}+2$ & $8.930 \mathrm{E}+1$ & $1.287 \mathrm{E}+2$ & $2.710 \mathrm{E}+1$ & $1.685 E+5$ & $5.189 \mathrm{E}+3$ & $4.725 \mathrm{E}+0$ \\
\hline \multicolumn{12}{|c|}{ Pin 29, State 2} \\
\hline 0 & $3.865 \mathrm{E}+1$ & $7.809 \mathrm{E}+0$ & 9.311E-1 & $2.731 E+1$ & $1.427 \mathrm{E}+2$ & $2.096 \mathrm{E}+2$ & $E+2$ & $\mathrm{E}+1$ & $E+5$ & $E+3$ & $1.789 \mathrm{E}+4$ \\
\hline 10 & $3.637 \mathrm{E}+1$ & $7.064 \mathrm{E}+0$ & 9.313E-1 & $2.329 \mathrm{E}+1$ & $1.165 E+2$ & $1.583 \mathrm{E}+2$ & $1.036 \mathrm{E}+2$ & $3.097 \mathrm{E}+1$ & $1.175 \mathrm{E}+5$ & $3.702 E+3$ & $9.029 \mathrm{E}+0$ \\
\hline 20 & $3.585 \mathrm{E}+1$ & $6.517 \mathrm{E}+0$ & 9.345E-1 & $2.309 \mathrm{E}+1$ & $1.105 \mathrm{E}+2$ & $1.218 \mathrm{E}+2$ & $1.002 \mathrm{E}+2$ & $3.053 E+1$ & $1.163 \mathrm{E}+5$ & $3.645 \mathrm{E}+3$ & $6.353 E+0$ \\
\hline 30 & $3.616 \mathrm{E}+1$ & $6.181 E+0$ & $9.387 \mathrm{E}-1$ & $2.343 E+1$ & $1.089 \mathrm{E}+2$ & $1.021 \mathrm{E}+2$ & $1.000 \mathrm{E}+2$ & $2.951 E+1$ & $1.186 \mathrm{E}+5$ & $3.700 \mathrm{E}+3$ & $5.363 \mathrm{E}+0$ \\
\hline 40 & $3.687 \mathrm{E}+1$ & $5.964 \mathrm{E}+0$ & $9.436 \mathrm{E}-1$ & $2.401 \mathrm{E}+1$ & $1.093 E+2$ & $9.070 \mathrm{E}+1$ & $1.014 \mathrm{E}+2$ & $2.809 E+1$ & $1.226 E+5$ & $3.808 \mathrm{E}+3$ & $4.805 E+0$ \\
\hline 50 & 3.772E+1 & $5.827 \mathrm{E}+0$ & $9.484 \mathrm{E}-1$ & $2.467 \mathrm{E}+1$ & $1.107 \mathrm{E}+2$ & $8.380 \mathrm{E}+1$ & $1.033 E+2$ & $2.650 \mathrm{E}+1$ & $1.271 E+5$ & $3.934 \mathrm{E}+3$ & $4.414 \mathrm{E}+0$ \\
\hline 60 & $3.849 E+1$ & $5.745 \mathrm{E}+0$ & $9.527 \mathrm{E}-1$ & $2.527 \mathrm{E}+1$ & $1.123 \mathrm{E}+2$ & $7.948 \mathrm{E}+1$ & $1.052 \mathrm{E}+2$ & $2.495 \mathrm{E}+1$ & $1.312 E+5$ & $4.049 \mathrm{E}+3$ & $4.102 \mathrm{E}+0$ \\
\hline \multicolumn{12}{|c|}{ Pin 72, State 1} \\
\hline 0 & 4.87 & $8.304 E+0$ & $9.966 \mathrm{E}-1$ & 3.5 & $1.801 \mathrm{E}+2$ & $.376 \mathrm{E}+2$ & +2 & $E+1$ & 1.7 & +3 & $5 E+4$ \\
\hline 10 & $4.590 \mathrm{E}+1$ & $7.589 \mathrm{E}+0$ & $9.972 \mathrm{E}-1$ & $2.988 E+1$ & $1.484 \mathrm{E}+2$ & $1.810 \mathrm{E}+2$ & $1.331 E+2$ & $3.404 \mathrm{E}+1$ & $1.615 E+5$ & $5.056 \mathrm{E}+3$ & $1.106 \mathrm{E}+1$ \\
\hline 20 & 4.543E+1 & $7.020 \mathrm{E}+0$ & $1.002 \mathrm{E}+0$ & $2.976 \mathrm{E}+1$ & 1.417E+2 & $1.406 \mathrm{E}+2$ & $1.295 E+2$ & $3.356 \mathrm{E}+1$ & $1.607 E+5$ & $5.006 E+3$ & $7.641 \mathrm{E}+0$ \\
\hline 30 & 4.607E+1 & $6.670 \mathrm{E}+0$ & $1.007 \mathrm{E}+0$ & $3.036 \mathrm{E}+1$ & $1.406 \mathrm{E}+2$ & $1.192 \mathrm{E}+2$ & $1.300 \mathrm{E}+2$ & $3.240 \mathrm{E}+1$ & $1.648 E+5$ & $5.109 \mathrm{E}+3$ & $6.397 \mathrm{E}+0$ \\
\hline 40 & $4.723 \mathrm{E}+1$ & $6.452 E+0$ & $1.014 \mathrm{E}+0$ & $3.129 \mathrm{E}+1$ & $1.421 E+2$ & $1.071 E+2$ & $1.325 \mathrm{E}+2$ & $3.079 \mathrm{E}+1$ & $1.711 E+5$ & $5.283 E+3$ & $5.703 E+0$ \\
\hline 50 & $4.853 \mathrm{E}+1$ & $6.318 \mathrm{E}+0$ & $1.020 \mathrm{E}+0$ & $3.231 \mathrm{E}+1$ & $1.446 \mathrm{E}+2$ & $9.988 \mathrm{E}+1$ & $6 \mathrm{E}+2$ & $2.902 \mathrm{E}+1$ & $1.781 \mathrm{E}+5$ & $5.478 \mathrm{E}+3$ & $5.222 \mathrm{E}+0$ \\
\hline 60 & $4.969 \mathrm{E}+1$ & $6.241 \mathrm{E}+0$ & $1.025 E+0$ & $3.321 E+1$ & $1.471 \mathrm{E}+2$ & $9.550 \mathrm{E}+1$ & $1.386 \mathrm{E}+2$ & $2.734 \mathrm{E}+1$ & $1.843 \mathrm{E}+5$ & $5.654 \mathrm{E}+3$ & $4.838 \mathrm{E}+0$ \\
\hline \multicolumn{12}{|c|}{ Pin 72, State 2} \\
\hline 0 & $3.087 \mathrm{E}+1$ & $7.387 \mathrm{E}+0$ & $9.079 \mathrm{E}-1$ & $2.142 \mathrm{E}+1$ & $1.115 \mathrm{E}+2$ & $1.805 E+2$ & $9.636 \mathrm{E}+1$ & $2.554 \mathrm{E}+$ & $9.115 \mathrm{E}+4$ & $2.899 \mathrm{E}+3$ & $1.294 \mathrm{E}+4$ \\
\hline 10 & $2.919 \mathrm{E}+1$ & $6.648 \mathrm{E}+0$ & $9.088 \mathrm{E}-1$ & $1.839 \mathrm{E}+1$ & $9.099 E+1$ & $1.340 \mathrm{E}+2$ & $8.079 \mathrm{E}+1$ & $2.771 E+1$ & $8.496 \mathrm{E}+4$ & $2.690 \mathrm{E}+3$ & $6.903 E+0$ \\
\hline 20 & $2.885 \mathrm{E}+1$ & $6.148 \mathrm{E}+0$ & $9.121 \mathrm{E}-1$ & $1.827 \mathrm{E}+1$ & $8.656 \mathrm{E}+1$ & $1.030 \mathrm{E}+2$ & $7.839 \mathrm{E}+1$ & $2.727 \mathrm{E}+1$ & $8.424 \mathrm{E}+4$ & $2.654 \mathrm{E}+3$ & $5.054 \mathrm{E}+0$ \\
\hline 30 & $2.912 \mathrm{E}+1$ & $5.847 \mathrm{E}+0$ & $9.163 \mathrm{E}-1$ & $1.853 \mathrm{E}+1$ & $8.546 \mathrm{E}+1$ & $8.672 \mathrm{E}+1$ & $7.830 \mathrm{E}+1$ & $2.632 \mathrm{E}+1$ & $8.599 \mathrm{E}+4$ & $2.696 \mathrm{E}+3$ & $4.350 \mathrm{E}+0$ \\
\hline 40 & $2.964 \mathrm{E}+1$ & $5.661 \mathrm{E}+0$ & $9.209 \mathrm{E}-1$ & $1.895 \mathrm{E}+1$ & $8.585 E+1$ & $7.749 \mathrm{E}+1$ & $7.927 \mathrm{E}+1$ & $2.501 E+1$ & $8.885 E+4$ & $2.773 E+3$ & $3.940 \mathrm{E}+0$ \\
\hline 50 & $3.024 \mathrm{E}+1$ & $5.549 \mathrm{E}+0$ & $9.253 \mathrm{E}-1$ & $1.940 \mathrm{E}+1$ & $8.685 \mathrm{E}+1$ & $7.197 \mathrm{E}+1$ & $8.062 E+1$ & $2.358 \mathrm{E}+1$ & $9.198 \mathrm{E}+4$ & $2.860 \mathrm{E}+3$ & $3.644 \mathrm{E}+0$ \\
\hline 60 & $3.076 \mathrm{E}+1$ & $5.485 \mathrm{E}+0$ & $9.291 \mathrm{E}-1$ & $1.980 \mathrm{E}+1$ & $8.789 \mathrm{E}+1$ & $6.857 \mathrm{E}+1$ & $8.188 E+1$ & $2.222 E+1$ & $9.473 E+4$ & $2.937 \mathrm{E}+3$ & $3.400 \mathrm{E}+0$ \\
\hline
\end{tabular}


Table 4.20. Fuel-averaged microscopic absorption cross section for Variant 12, States 1 and 2

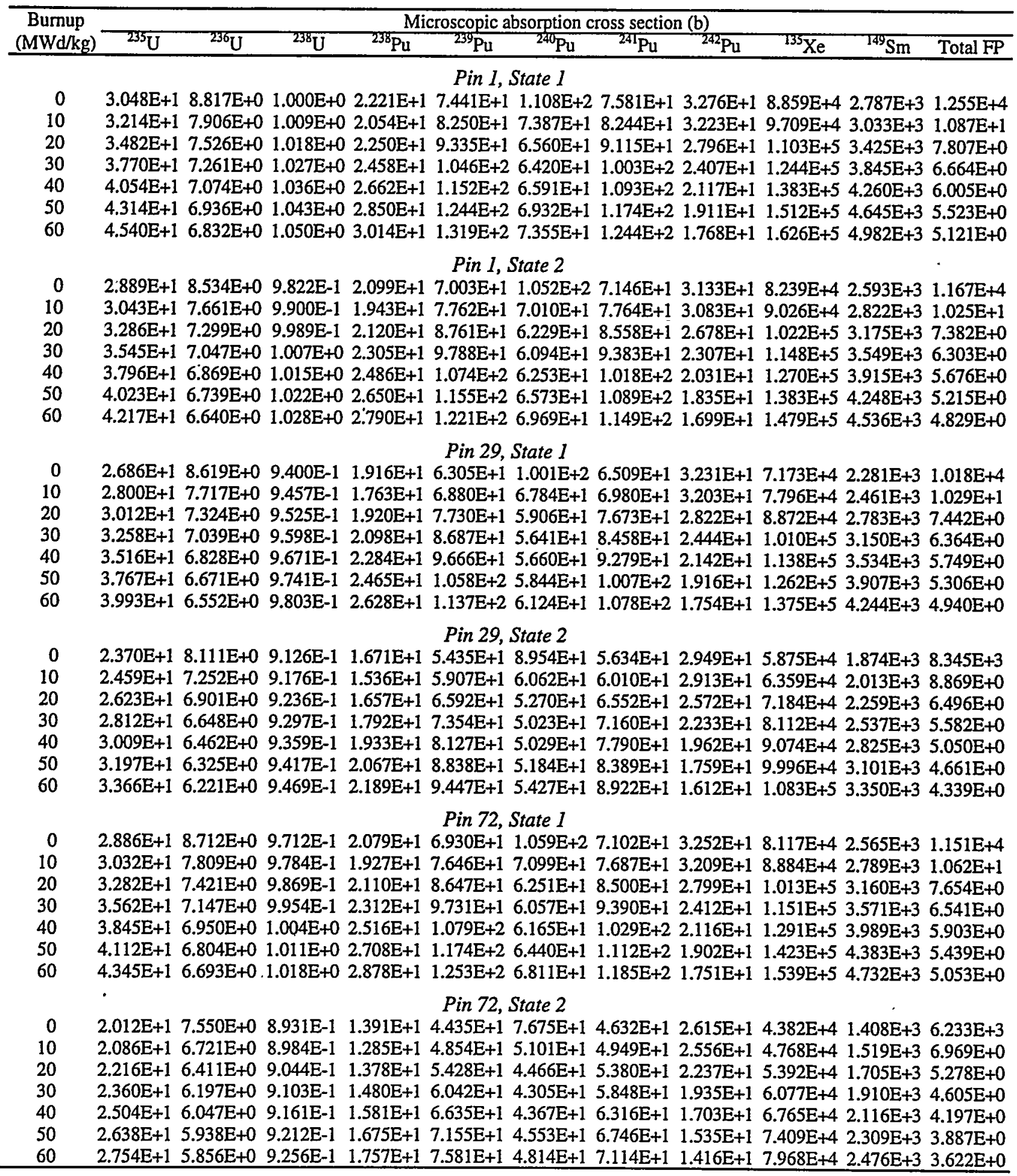


Table 4.21. Cell relative production reaction rate for uranium and plutonium isotopes vs burnup for Variants 1-4 and 7-10

\begin{tabular}{|c|c|c|c|c|c|c|c|c|}
\hline \multirow{2}{*}{$\begin{array}{c}\text { Burnup } \\
\text { (MWd/kg) }\end{array}$} & \multicolumn{8}{|c|}{ Relative production rate } \\
\hline & ${ }^{235} \mathrm{U}$ & ${ }^{236} \mathrm{U}$ & ${ }^{238} \mathrm{U}$ & ${ }^{238} \mathrm{Pu}$ & ${ }^{239} \mathrm{Pu}$ & ${ }^{240} \mathrm{Pu}$ & ${ }^{241} \mathrm{Pu}$ & ${ }^{242} \mathrm{Pu}$ \\
\hline \multicolumn{9}{|c|}{ Variant 1} \\
\hline 0 & 9.334E-1 & 0.0 & $6.664 \mathrm{E}-2$ & 0.0 & 0.0 & 0.0 & 0.0 & 0.0 \\
\hline 10 & $6.912 \mathrm{E}-1$ & $4.191 \mathrm{E}-4$ & $7.311 \mathrm{E}-2$ & $9.024 \mathrm{E}-6$ & $2.232 \mathrm{E}-1$ & $2.226 \mathrm{E}-4$ & $1.170 \mathrm{E}-2$ & $3.775 \mathrm{E}-6$ \\
\hline 20 & $5.381 \mathrm{E}-1$ & 7.771E-4 & $7.900 \mathrm{E}-2$ & $5.932 \mathrm{E}-5$ & $3.361 \mathrm{E}-1$ & $6.003 \mathrm{E}-4$ & $4.499 \mathrm{E}-2$ & $3.434 \mathrm{E}-5$ \\
\hline 30 & $4.202 \mathrm{E}-1$ & $1.080 \mathrm{E}-3$ & $8.419 \mathrm{E}-2$ & $1.810 \mathrm{E}-4$ & $4.081 \mathrm{E}-1$ & $1.007 \mathrm{E}-3$ & $8.451 \mathrm{E}-2$ & $1.072 \mathrm{E}-4$ \\
\hline 40 & $3.232 \mathrm{E}-1$ & $1.332 \mathrm{E}-3$ & 8.917E-2 & $3.963 \mathrm{E}-4$ & $4.601 \mathrm{E}-1$ & $1.406 \mathrm{E}-3$ & $1.229 \mathrm{E}-1$ & $2.231 \mathrm{E}-4$ \\
\hline 50 & $2.423 \mathrm{E}-1$ & $1.531 \mathrm{E}-3$ & 9.397E-2 & $7.128 \mathrm{E}-4$ & $5.002 \mathrm{E}-1$ & $1.776 \mathrm{E}-3$ & $1.567 \mathrm{E}-1$ & $3.759 \mathrm{E}-4$ \\
\hline 60 & $1.758 \mathrm{E}-1$ & $1.674 \mathrm{E}-3$ & $9.828 \mathrm{E}-2$ & $1.117 \mathrm{E}-3$ & 5.317E-1 & $2.102 E-3$ & $1.846 \mathrm{E}-1$ & $5.552 \mathrm{E}-4$ \\
\hline \multicolumn{9}{|c|}{ Variant 2} \\
\hline 0 & $2.254 \mathrm{E}-2$ & 0.0 & 7.331E-2 & 0.0 & $8.914 \mathrm{E}-1$ & $1.033 E-3$ & $1.172 \mathrm{E}-2$ & 0.0 \\
\hline 10 & $2.132 \mathrm{E}-2$ & $1.751 \mathrm{E}-5$ & 8.095E-2 & $6.143 E-6$ & $8.227 \mathrm{E}-1$ & $2.552 \mathrm{E}-3$ & $7.225 \mathrm{E}-2$ & $4.775 \mathrm{E}-5$ \\
\hline 20 & $1.947 \mathrm{E}-2$ & $3.421 \mathrm{E}-5$ & 8.567E-2 & $3.621 E-5$ & $7.481 \mathrm{E}-1$ & $3.586 \mathrm{E}-3$ & $1.422 \mathrm{E}-1$ & $1.738 \mathrm{E}-4$ \\
\hline 30 & $1.717 \mathrm{E}-2$ & $4.903 \mathrm{E}-5$ & $8.976 \mathrm{E}-2$ & $1.071 E-4$ & $6.809 \mathrm{E}-1$ & $4.197 \mathrm{E}-3$ & $2.058 E-1$ & $3.751 \mathrm{E}-4$ \\
\hline 40 & $1.458 \mathrm{E}-2$ & $6.146 \mathrm{E}-5$ & $9.346 \mathrm{E}-2$ & $2.286 \mathrm{E}-4$ & $6.296 \mathrm{E}-1$ & $4.459 \mathrm{E}-3$ & $2.539 \mathrm{E}-1$ & $6.340 \mathrm{E}-4$ \\
\hline 50 & $1.189 \mathrm{E}-2$ & $7.104 \mathrm{E}-5$ & $9.687 \mathrm{E}-2$ & $3.965 \mathrm{E}-4$ & $5.971 \mathrm{E}-1$ & $4.469 \mathrm{E}-3$ & $2.830 \mathrm{E}-1$ & $9.237 \mathrm{E}-4$ \\
\hline 60 & 9.313E-3 & $7.761 \mathrm{E}-5$ & $9.990 \mathrm{E}-2$ & $5.930 \mathrm{E}-4$ & $5.808 \mathrm{E}-1$ & $4.330 \mathrm{E}-3$ & $2.954 \mathrm{E}-1$ & $1.214 \mathrm{E}-3$ \\
\hline \multicolumn{9}{|c|}{ Variant 3} \\
\hline 0 & $4.261 \mathrm{E}-1$ & $9.712 \mathrm{E}-4$ & $7.827 \mathrm{E}-2$ & $6.333 \mathrm{E}-4$ & $4.160 \mathrm{E}-1$ & $9.890 \mathrm{E}-4$ & $7.623 E-2$ & $9.886 \mathrm{E}-5$ \\
\hline \multicolumn{9}{|c|}{ Variant 4} \\
\hline 0 & $4.240 \mathrm{E}-1$ & $1.018 \mathrm{E}-3$ & $8.306 \mathrm{E}-2$ & $6.570 \mathrm{E}-4$ & $4.136 \mathrm{E}-1$ & $1.047 \mathrm{E}-3$ & $7.574 \mathrm{E}-2$ & $1.049 \mathrm{E}-4$ \\
\hline \multicolumn{9}{|c|}{ Variant 7} \\
\hline 0 & $2.285 \mathrm{E}-2$ & 0.0 & $6.792 \mathrm{E}-2$ & 0.0 & $9.092 \mathrm{E}-1$ & 0.0 & 0.0 & 0.0 \\
\hline \multicolumn{9}{|c|}{ Variant 8} \\
\hline 0 & $9.219 \mathrm{E}-1$ & 0.0 & 7.694E-2 & 0.0 & 0.0 & $1.118 \mathrm{E}-3$ & 0.0 & 0.0 \\
\hline \multicolumn{9}{|c|}{ Variant 9} \\
\hline 0 & $1.874 \mathrm{E}-2$ & 0.0 & $5.388 \mathrm{E}-2$ & 0.0 & 0.0 & 0.0 & $9.274 \mathrm{E}-1$ & 0.0 \\
\hline \multicolumn{9}{|c|}{ Variant 10} \\
\hline 0 & $1.626 \mathrm{E}-2$ & 0.0 & $8.643 E-2$ & $1.971 \mathrm{E}-3$ & $7.171 \mathrm{E}-1$ & $1.188 \mathrm{E}-2$ & $1.636 \mathrm{E}-1$ & $1.995 \mathrm{E}-3$ \\
\hline 10 & $1.479 \mathrm{E}-2$ & $1.758 \mathrm{E}-5$ & $8.986 \mathrm{E}-2$ & $2.045 \mathrm{E}-3$ & $6.765 \mathrm{E}-1$ & $1.237 \mathrm{E}-2$ & $1.982 \mathrm{E}-1$ & $2.111 \mathrm{E}-3$ \\
\hline 20 & $1.343 \mathrm{E}-2$ & $3.311 \mathrm{E}-5$ & 9.193E-2 & $2.245 E-3$ & $6.451 \mathrm{E}-1$ & $1.255 \mathrm{E}-2$ & $2.261 E-1$ & $2.238 \mathrm{E}-3$ \\
\hline 30 & $1.215 \mathrm{E}-2$ & $4.675 \mathrm{E}-5$ & 9.393E-2 & $2.508 \mathrm{E}-3$ & $6.191 \mathrm{E}-1$ & $1.260 \mathrm{E}-2$ & $2.485 \mathrm{E}-1$ & 2.397E-3 \\
\hline 40 & $1.094 \mathrm{E}-2$ & $5.861 \mathrm{E}-5$ & $9.588 \mathrm{E}-2$ & $2.808 \mathrm{E}-3$ & 5.973E-1 & $1.254 \mathrm{E}-2$ & 2.662E-1 & $2.580 \mathrm{E}-3$ \\
\hline 50 & $9.787 \mathrm{E}-3$ & $6.872 \mathrm{E}-5$ & $9.768 \mathrm{E}-2$ & $3.135 \mathrm{E}-3$ & $5.792 \mathrm{E}-1$ & $1.237 \mathrm{E}-2$ & $2.798 \mathrm{E}-1$ & $2.778 \mathrm{E}-3$ \\
\hline 60 & 8.695E-3 & $7.718 \mathrm{E}-5$ & $9.946 \mathrm{E}-2$ & $3.478 \mathrm{E}-3$ & $5.642 \mathrm{E}-1$ & $1.212 \mathrm{E}-2$ & $2.898 \mathrm{E}-1$ & $2.987 \mathrm{E}-3$ \\
\hline
\end{tabular}


Table 4.22. Cell relative production reaction rates for Variant 11, States 1 and 2

\begin{tabular}{|c|c|c|c|c|c|c|c|c|}
\hline \multirow{2}{*}{$\begin{array}{c}\text { Burnup } \\
\text { (MWd/kg) }\end{array}$} & \multicolumn{8}{|c|}{ Relative production rate } \\
\hline & ${ }^{235} \mathrm{U}$ & ${ }^{236} \mathrm{U}$ & ${ }^{238} \mathrm{U}$ & ${ }^{238} \mathrm{Pu}$ & ${ }^{239} \mathrm{Pu}$ & ${ }^{240} \mathrm{Pu}$ & ${ }^{241} \mathrm{Pu}$ & ${ }^{242} \mathrm{Pu}$ \\
\hline \multicolumn{9}{|c|}{ Pin 1, State 1} \\
\hline 0 & $9.425 \mathrm{E}-1$ & 0.0 & $5.748 \mathrm{E}-2$ & 0.0 & 0.0 & 0.0 & 0.0 & 0.0 \\
\hline 10 & $7.091 \mathrm{E}-1$ & $3.700 \mathrm{E}-4$ & $6.292 \mathrm{E}-2$ & $7.523 \mathrm{E}-6$ & $2.156 \mathrm{E}-1$ & $1.934 \mathrm{E}-4$ & $1.174 \mathrm{E}-2$ & $3.368 \mathrm{E}-6$ \\
\hline 20 & $5.554 \mathrm{E}-1$ & $6.917 \mathrm{E}-4$ & $6.848 \mathrm{E}-2$ & $5.075 \mathrm{E}-5$ & $3.276 \mathrm{E}-1$ & $5.243 E-4$ & $4.697 \mathrm{E}-2$ & $3.201 \mathrm{E}-5$ \\
\hline 30 & $4.318 \mathrm{E}-1$ & $9.722 \mathrm{E}-4$ & $7.397 \mathrm{E}-2$ & $1.596 \mathrm{E}-4$ & $4.013 \mathrm{E}-1$ & $8.784 E-4$ & $9.021 \mathrm{E}-2$ & $1.034 \mathrm{E}-4$ \\
\hline 40 & $3.260 \mathrm{E}-1$ & $1.211 \mathrm{E}-3$ & $7.952 \mathrm{E}-2$ & $3.593 E-4$ & $4.574 \mathrm{E}-1$ & $1.221 \mathrm{E}-3$ & $1.328 \mathrm{E}-1$ & $2.212 \mathrm{E}-4$ \\
\hline 50 & $2.348 \mathrm{E}-1$ & $1.402 \mathrm{E}-3$ & $8.495 \mathrm{E}-2$ & $6.601 E-4$ & $5.034 \mathrm{E}-1$ & $1.531 \mathrm{E}-3$ & $1.705 \mathrm{E}-1$ & $3.805 \mathrm{E}-4$ \\
\hline 60 & $1.594 \mathrm{E}-1$ & $1.536 \mathrm{E}-3$ & $8.986 \mathrm{E}-2$ & $1.045 E-3$ & $5.408 \mathrm{E}-1$ & $1.792 \mathrm{E}-3$ & $2.006 \mathrm{E}-1$ & $5.694 \mathrm{E}-4$ \\
\hline \multicolumn{9}{|c|}{ Pin 1, State 2} \\
\hline 0 & $9.350 \mathrm{E}-1$ & 0.0 & $6.500 \mathrm{E}-2$ & 0.0 & 0.0 & 0.0 & 0.0 & 0.0 \\
\hline 10 & $7.036 \mathrm{E}-1$ & $4.068 \mathrm{E}-4$ & $7.064 \mathrm{E}-2$ & $7.952 \mathrm{E}-6$ & 2.134E-1 & $2.150 \mathrm{E}-4$ & 1.162E-2 & $3.770 \mathrm{E}-6$ \\
\hline 20 & $5.508 \mathrm{E}-1$ & $7.601 \mathrm{E}-4$ & $7.674 \mathrm{E}-2$ & $5.358 \mathrm{E}-5$ & 3.243E-1 & $5.824 \mathrm{E}-4$ & $4.646 \mathrm{E}-2$ & $3.577 \mathrm{E}-5$ \\
\hline 30 & $4.279 \mathrm{E}-1$ & $1.070 \mathrm{E}-3$ & $8.290 \mathrm{E}-2$ & $1.683 \mathrm{E}-4$ & $3.970 \mathrm{E}-1$ & $9.765 \mathrm{E}-4$ & $8.918 \mathrm{E}-2$ & $1.156 \mathrm{E}-4$ \\
\hline 40. & 3.227E-1 & $1.334 \mathrm{E}-3$ & $8.923 \mathrm{E}-2$ & $3.789 \mathrm{E}-4$ & $4.523 \mathrm{E}-1$ & $1.360 \mathrm{E}-3$ & $1.312 \mathrm{E}-1$ & $2.475 E-4$ \\
\hline 50 & 2.322E-1 & $1.547 \mathrm{E}-3$ & $9.549 \mathrm{E}-2$ & $6.959 \mathrm{E}-4$ & $4.973 \mathrm{E}-1$ & $1.708 \mathrm{E}-3$ & $1.682 \mathrm{E}-1$ & $4.265 \mathrm{E}-4$ \\
\hline 60 & $1.574 \mathrm{E}-1$ & $1.698 \mathrm{E}-3$ & $1.012 \mathrm{E}-1$ & $1.101 \mathrm{E}-3$ & $5.339 \mathrm{E}-1$ & $2.003 \mathrm{E}-3$ & $1.977 \mathrm{E}-1$ & $6.394 \mathrm{E}-4$ \\
\hline \multicolumn{9}{|c|}{ Pin 29, State 1} \\
\hline 0 & 9.363E-1 & 0.0 & $6.374 \mathrm{E}-2$ & 0.0 & 0.0 & 0.0 & 0.0 & 0.0 \\
\hline 10 & $7.156 \mathrm{E}-1$ & $3.748 \mathrm{E}-4$ & $6.948 \mathrm{E}-2$ & $7.051 \mathrm{E}-6$ & $2.044 \mathrm{E}-1$ & $1.877 \mathrm{E}-4$ & $9.827 \mathrm{E}-3$ & $2.842 E-6$ \\
\hline 20 & $5.700 \mathrm{E}-1$ & $7.026 \mathrm{E}-4$ & $7.497 \mathrm{E}-2$ & $4.654 \mathrm{E}-5$ & $3.136 \mathrm{E}-1$ & $5.201 \mathrm{E}-4$ & $3.991 \mathrm{E}-2$ & $2.729 \mathrm{E}-5$ \\
\hline 30 & $4.533 \mathrm{E}-1$ & $9.905 \mathrm{E}-4$ & 8.019E-2 & $1.448 \mathrm{E}-4$ & $3.858 \mathrm{E}-1$ & $8.903 E-4$ & 7.797E-2 & 8.919E-5 \\
\hline 40 & $3.529 \mathrm{E}-1$ & $1.240 \mathrm{E}-3$ & 8.545E-2 & $3.256 \mathrm{E}-4$ & $4.405 \mathrm{E}-1$ & $1.264 \mathrm{E}-3$ & $1.170 \mathrm{E}-1$ & $1.936 \mathrm{E}-4$ \\
\hline 50 & $2.648 \mathrm{E}-1$ & $1.447 \mathrm{E}-3$ & $9.077 \mathrm{E}-2$ & $6.026 \mathrm{E}-4$ & $4.851 \mathrm{E}-1$ & $1.618 \mathrm{E}-3$ & $1.533 E-1$ & $3.389 \mathrm{E}-4$ \\
\hline 60 & $1.892 \mathrm{E}-1$ & $1.606 \mathrm{E}-3$ & $9.586 \mathrm{E}-2$ & $9.693 \mathrm{E}-4$ & $5.221 \mathrm{E}-1$ & $1.933 \mathrm{E}-3$ & $1.842 \mathrm{E}-1$ & $5.180 \mathrm{E}-4$ \\
\hline \multicolumn{9}{|c|}{ Pin 29, State 2} \\
\hline 0 & $9.216 \mathrm{E}-1$ & 0.0 & $7.840 \mathrm{E}-2$ & 0.0 & 0.0 & 0.0 & 0.0 & 0.0 \\
\hline 10 & 7.049E-1 & $4.479 \mathrm{E}-4$ & $8.484 \mathrm{E}-2$ & 7.977E-6 & $1.999 \mathrm{E}-1$ & $2.282 E-4$ & 9.599E-3. & $3.483 E-6$ \\
\hline 20 & 5.612E-1 & $8.403 E-4$ & $9.143 \mathrm{E}-2$ & $5.263 \mathrm{E}-5$ & $3.066 \mathrm{E}-1$ & $6.327 \mathrm{E}-4$ & 3.897E-2 & 3.340E-5 \\
\hline 30 & $4.458 \mathrm{E}-1$ & $1.188 \mathrm{E}-3$ & $9.793 \mathrm{E}-2$ & $1.637 \mathrm{E}-4$ & $3.770 \mathrm{E}-1$ & $1.085 \mathrm{E}-3$ & $7.608 \mathrm{E}-2$ & $1.093 \mathrm{E}-4$ \\
\hline 40 & $3.465 \mathrm{E}-1$ & $1.492 \mathrm{E}-3$ & $1.046 \mathrm{E}-1$ & $3.681 \mathrm{E}-4$ & 4.300E-1 & $1.546 \mathrm{E}-3$ & 1.140E-1 & $2.378 E-4$ \\
\hline 50 & 2.595E-1 & $1.747 \mathrm{E}-3$ & $1.115 \mathrm{E}-1$ & $6.810 \mathrm{E}-4$ & 4.729E-1 & $1.985 \mathrm{E}-3$ & $1.491 \mathrm{E}-1$ & $4.177 \mathrm{E}-4$ \\
\hline 60 & $1.850 \mathrm{E}-1$ & $1.944 \mathrm{E}-3$ & 1.181E-1 & $1.095 \mathrm{E}-3$ & $5.082 \mathrm{E}-1$ & $2.379 \mathrm{E}-3$ & $1.789 \mathrm{E}-1$ & $6.401 \mathrm{E}-4$ \\
\hline \multicolumn{9}{|c|}{ Pin 72 , State 1} \\
\hline 0 & $9.401 \mathrm{E}-1$ & 0.0 & $5.989 \mathrm{E}-2$ & 0.0 & 0.0 & 0.0 & 0.0 & 0.0 \\
\hline 10 & $7.125 \mathrm{E}-1$ & $3.718 \mathrm{E}-4$ & $6.542 \mathrm{E}-2$ & $7.316 \mathrm{E}-6$ & $2.106 \mathrm{E}-1$ & $1.911 \mathrm{E}-4$ & $1.090 \mathrm{E}-2$ & $3.144 \mathrm{E}-6$ \\
\hline 20 & 5.623E-1 & $6.958 \mathrm{E}-4$ & $7.094 \mathrm{E}-2$ & 4.893E-5 & 3.213E-1 & $5.232 \mathrm{E}-4$ & $4.390 \mathrm{E}-2$ & $2.999 \mathrm{E}-5$ \\
\hline 30 & 4.415E-1 & $9.791 E-4$ & $7.631 \mathrm{E}-2$ & $1.533 \mathrm{E}-4$ & 3.944E-1 & $8.849 \mathrm{E}-4$ & $8.500 \mathrm{E}-2$ & $9.736 \mathrm{E}-5$ \\
\hline 40 & 3.377E-1 & $1.222 \mathrm{E}-3$ & $8.174 \mathrm{E}-2$ & $3.451 \mathrm{E}-4$ & $4.500 \mathrm{E}-1$ & $1.241 \mathrm{E}-3$ & $1.263 \mathrm{E}-1$ & $2.097 \mathrm{E}-4$ \\
\hline 50 & $2.474 \mathrm{E}-1$ & $1.420 \mathrm{E}-3$ & $8.715 \mathrm{E}-2$ & $6.364 \mathrm{E}-4$ & $4.956 \mathrm{E}-1$ & $1.570 \mathrm{E}-3$ & $1.635 \mathrm{E}-1$ & $3.637 \mathrm{E}-4$ \\
\hline 60 & $1.714 \mathrm{E}-1$ & $1.565 \mathrm{E}-3$ & $9.219 \mathrm{E}-2$ & $1.014 \mathrm{E}-3$ & 5.332E-1 & $1.854 \mathrm{E}-3$ & $1.943 \mathrm{E}-1$ & $5.496 \mathrm{E}-4$ \\
\hline \multicolumn{9}{|c|}{ Pin 72, State 2} \\
\hline 0 & 9.019E-1 & 0.0 & $9.811 \mathrm{E}-2$ & 0.0 & 0.0 & 0.0 & 0.0 & 0.0 \\
\hline 10 & $6.839 \mathrm{E}-1$ & $5.788 \mathrm{E}-4$ & $1.063 \mathrm{E}-1$ & $1.010 \mathrm{E}-5$ & $1.985 \mathrm{E}-1$ & $3.091 \mathrm{E}-4$ & $1.025 \mathrm{E}-2$ & $5.162 \mathrm{E}-6$ \\
\hline 20 & 5.391E-1 & $1.086 \mathrm{E}-3$ & $1.152 \mathrm{E}-1$ & $6.757 \mathrm{E}-5$ & $3.021 \mathrm{E}-1$ & $8.486 \mathrm{E}-4$ & $4.120 \mathrm{E}-2$ & $4.921 \mathrm{E}-5$ \\
\hline 30 & $4.222 \mathrm{E}-1$ & $1.536 \mathrm{E}-3$ & 1.243E-1 & $2.114 \mathrm{E}-4$ & 3.699E-1 & $1.442 \mathrm{E}-3$ & $7.957 \mathrm{E}-2$ & $1.602 \mathrm{E}-4$ \\
\hline 40 & $3.215 \mathrm{E}-1$ & $1.927 \mathrm{E}-3$ & 1.337E-1 & $4.751 \mathrm{E}-4$ & $4.208 \mathrm{E}-1$ & $2.033 \mathrm{E}-3$ & $1.178 \mathrm{E}-1$ & $3.465 \mathrm{E}-4$ \\
\hline 50 & 2.344E-1 & $2.249 \mathrm{E}-3$ & $1.432 \mathrm{E}-1$ & 8.737E-4 & 4.617E-1 & $2.584 \mathrm{E}-3$ & $1.519 \mathrm{E}-1$ & $6.034 \mathrm{E}-4$ \\
\hline 60 & $1.615 \mathrm{E}-1$ & $2.487 \mathrm{E}-3$ & $1.519 \mathrm{E}-1$ & $1.389 \mathrm{E}-3$ & $4.947 \mathrm{E}-1$ & $3.060 \mathrm{E}-3$ & $1.796 \mathrm{E}-1$ & $9.146 \mathrm{E}-4$ \\
\hline
\end{tabular}


Table 4.23. Cell relative production reaction rates for Variant 12, States 1 and 2

\begin{tabular}{|c|c|c|c|c|c|c|c|c|}
\hline \multirow{2}{*}{$\begin{array}{c}\text { Burnup } \\
(\mathrm{MWd} / \mathrm{kg})\end{array}$} & \multicolumn{8}{|c|}{ Relative production rate } \\
\hline & ${ }^{235} \mathrm{U}$ & ${ }^{236} \mathrm{U}$ & ${ }^{238} \mathrm{U}$ & ${ }^{238} \mathrm{Pu}$ & ${ }^{239} \mathrm{Pu}$ & ${ }^{240} \mathrm{Pu}$ & ${ }^{241} \mathrm{Pu}$ & ${ }^{242} \mathrm{Pu}$ \\
\hline \multicolumn{9}{|c|}{ Pin 1, State 1} \\
\hline 0 & $2.221 \mathrm{E}-2$ & 0.0 & $6.066 \mathrm{E}-2$ & 0.0 & $9.046 \mathrm{E}-1$ & $8.400 \mathrm{E}-4$ & $1.174 \mathrm{E}-2$ & 0.0 \\
\hline 10 & $2.189 \mathrm{E}-2$ & $1.439 \mathrm{E}-5$ & $6.849 \mathrm{E}-2$ & $4.942 \mathrm{E}-6$ & 8.298E-1 & $2.290 \mathrm{E}-3$ & $7.728 \mathrm{E}-2$ & $4.621 \mathrm{E}-5$ \\
\hline 20 & $2.069 \mathrm{E}-2$ & $2.903 \mathrm{E}-5$ & $7.424 \mathrm{E}-2$ & $3.122 \mathrm{E}-5$ & $7.413 \mathrm{E}-1$ & $3.267 \mathrm{E}-3$ & $1.596 \mathrm{E}-1$ & $1.798 \mathrm{E}-4$ \\
\hline 30 & $1.854 \mathrm{E}-2$ & $4.289 \mathrm{E}-5$ & $7.952 \mathrm{E}-2$ & $9.748 \mathrm{E}-5$ & $6.599 \mathrm{E}-1$ & $3.779 \mathrm{E}-3$ & 2.361E-1 & $4.105 \mathrm{E}-4$ \\
\hline 40 & $1.558 \mathrm{E}-2$ & $5.497 \mathrm{E}-5$ & $8.426 \mathrm{E}-2$ & $2.144 \mathrm{E}-4$ & $6.025 \mathrm{E}-1$ & $3.884 \mathrm{E}-3$ & $2.897 \mathrm{E}-1$ & $7.213 \mathrm{E}-4$ \\
\hline 50 & $1.219 \mathrm{E}-2$ & $6.443 E-5$ & $8.836 \mathrm{E}-2$ & $3.730 \mathrm{E}-4$ & $5.744 \mathrm{E}-1$ & $3.718 \mathrm{E}-3$ & $3.146 \mathrm{E}-1$ & $1.073 \mathrm{E}-3$ \\
\hline 60 & 8.905E-3 & $7.086 \mathrm{E}-5$ & $9.178 \mathrm{E}-2$ & $5.464 \mathrm{E}-4$ & $5.688 \mathrm{E}-1$ & $3.434 \mathrm{E}-3$ & $3.168 \mathrm{E}-1$ & $1.423 \mathrm{E}-3$ \\
\hline \multicolumn{9}{|c|}{ Pin 1, State 2} \\
\hline 0 & $2.218 \mathrm{E}-2$ & 0.0 & $6.631 \mathrm{E}-2$ & 0.0 & 8.989E-1 & $9.120 \mathrm{E}-4$ & $1.169 \mathrm{E}-2$ & 0.0 \\
\hline 10 & $2.183 \mathrm{E}-2$ & $1.543 \mathrm{E}-5$ & $7.480 \mathrm{E}-2$ & $5.190 \mathrm{E}-6$ & $8.238 \mathrm{E}-1$ & $2.486 \mathrm{E}-3$ & 7.683E-2 & $5.035 \mathrm{E}-5$ \\
\hline 20 & $2.059 \mathrm{E}-2$ & $3.121 E-5$ & $8.128 \mathrm{E}-2$ & 3.277E-5 & 7.352E-1 & 3.557E-3 & $1.584 \mathrm{E}-1$ & $1.963 \mathrm{E}-4$ \\
\hline 30 & $1.843 \mathrm{E}-2$ & $4.628 \mathrm{E}-5$ & $8.736 \mathrm{E}-2$ & $1.023 \mathrm{E}-4$ & $6.539 \mathrm{E}-1$ & $4.127 \mathrm{E}-3$ & $2.340 \mathrm{E}-1$ & $4.498 \mathrm{E}-4$ \\
\hline 40 & $1.546 \mathrm{E}-2$ & $5.956 \mathrm{E}-5$ & $9.294 \mathrm{E}-2$ & $2.251 \mathrm{E}-4$ & $5.965 \mathrm{E}-1$ & $4.259 \mathrm{E}-3$ & $2.867 \mathrm{E}-1$ & $7.936 \mathrm{E}-4$ \\
\hline 50 & $1.208 \mathrm{E}-2$ & $7.009 \mathrm{E}-5$ & $9.786 \mathrm{E}-2$ & $3.919 \mathrm{E}-4$ & 5.681E-1 & $4.093 E-3$ & $3.110 \mathrm{E}-1$ & $1.186 \mathrm{E}-3$ \\
\hline 60 & 8.811E-3 & $7.740 \mathrm{E}-5$ & $1.021 \mathrm{E}-1$ & $5.744 \mathrm{E}-4$ & 5.621E-1 & 3.795E-3 & $3.128 \mathrm{E}-1$ & $1.578 \mathrm{E}-3$ \\
\hline \multicolumn{9}{|c|}{ Pin 29, State I } \\
\hline 0 & $2.259 \mathrm{E}-2$ & 0.0 & $7.092 \mathrm{E}-2$ & 0.0 & 8.937E-1 & $9.854 \mathrm{E}-4$ & $1.177 \mathrm{E}-2$ & 0.0 \\
\hline 10 & $2.182 \mathrm{E}-2$ & $1.483 \mathrm{E}-5$ & 7.773E-2 & $4.884 \mathrm{E}-6$ & 8.313E-1 & $2.403 \mathrm{E}-3$ & $6.649 \mathrm{E}-2$ & $4.059 \mathrm{E}-5$ \\
\hline 20 & $2.050 \mathrm{E}-2$ & 2.938E-5 & 8.202E-2 & $2.927 \mathrm{E}-5$ & $7.590 \mathrm{E}-1$ & $3.424 \mathrm{E}-3$ & $1.342 \mathrm{E}-1$ & $1.515 \mathrm{E}-4$ \\
\hline 30 & $1.863 \mathrm{E}-2$ & 4.301E-5 & $8.600 \mathrm{E}-2$ & 8.889E-5 & $6.878 \mathrm{E}-1$ & $4.070 \mathrm{E}-3$ & $2.016 \mathrm{E}-1$ & $3.432 \mathrm{E}-4$ \\
\hline 40 & $1.621 \mathrm{E}-2$ & $5.527 \mathrm{E}-5$ & $8.996 \mathrm{E}-2$ & $1.956 \mathrm{E}-4$ & $6.287 \mathrm{E}-1$ & $4.364 \mathrm{E}-3$ & 2.573E-1 & $6.127 \mathrm{E}-4$ \\
\hline 50 & $1.336 \mathrm{E}-2$ & $6.555 \mathrm{E}-5$ & $9.383 \mathrm{E}-2$ & $3.485 \mathrm{E}-4$ & $5.892 \mathrm{E}-1$ & $4.368 \mathrm{E}-3$ & $2.935 \mathrm{E}-1$ & $9.402 \mathrm{E}-4$ \\
\hline 60 & $1.037 \mathrm{E}-2$ & 7.334E-5 & $9.740 \mathrm{E}-2$ & $5.294 \mathrm{E}-4$ & $5.705 \mathrm{E}-1$ & $4.181 \mathrm{E}-3$ & $3.088 \mathrm{E}-1$ & $1.292 \mathrm{E}-3$ \\
\hline \multicolumn{9}{|c|}{ Pin 29, State 2} \\
\hline 0 & $2.264 \mathrm{E}-2$ & 0.0 & $8.300 \mathrm{E}-2$ & 0.0 & $8.815 \mathrm{E}-1$ & $1.153 \mathrm{E}-3$ & $1.169 \mathrm{E}-2$ & 0.0 \\
\hline 10 & $2.181 \mathrm{E}-2$ & $1.704 \mathrm{E}-5$ & $9.117 \mathrm{E}-2$ & $5.495 \mathrm{E}-6$ & $8.182 \mathrm{E}-1$ & $2.820 \mathrm{E}-3$ & $6.578 \mathrm{E}-2$ & $4.776 \mathrm{E}-5$ \\
\hline 20 & 2.042E-2 & 3.401E-5 & $9.681 \mathrm{E}-2$ & $3.297 \mathrm{E}-5$ & 7.455E-1 & $4.045 \mathrm{E}-3$ & $1.324 \mathrm{E}-1$ & $1.794 \mathrm{E}-4$ \\
\hline 30 & $1.848 \mathrm{E}-2$ & 5.019E-5 & $1.022 \mathrm{E}-1$ & $1.003 \mathrm{E}-4$ & $6.743 \mathrm{E}-1$ & $4.843 E-3$ & $1.981 \mathrm{E}-1$ & $4.093 \mathrm{E}-4$ \\
\hline 40 & $1.602 \mathrm{E}-2$ & $6.497 \mathrm{E}-5$ & $1.077 \mathrm{E}-1$ & $2.208 \mathrm{E}-4$ & $6.152 \mathrm{E}-1$ & $5.229 \mathrm{E}-3$ & $2.521 \mathrm{E}-1$ & $7.361 \mathrm{E}-4$ \\
\hline 50 & 1.317E-2 & 7.762E-5 & 1.131E-1 & $3.936 \mathrm{E}-4$ & $5.757 \mathrm{E}-1$ & $5.270 \mathrm{E}-3$ & $2.867 \mathrm{E}-1$ & $1.137 \mathrm{E}-3$ \\
\hline 60 & $1.020 \mathrm{E}-2$ & $8.735 \mathrm{E}-5$ & $1.181 \mathrm{E}-1$ & $5.980 \mathrm{E}-4$ & $5.565 \mathrm{E}-1$ & $5.073 E-3$ & $3.009 \mathrm{E}-1$ & $1.572 \mathrm{E}-3$ \\
\hline \multicolumn{9}{|c|}{ Pin 72, State 1} \\
\hline 0 & 2.237E-2 & 0.0 & $6.477 \mathrm{E}-2$ & 0.0 & $9.002 \mathrm{E}-1$ & $8.996 \mathrm{E}-4$ & $1.176 \mathrm{E}-2$ & 0.0 \\
\hline 10 & $2.187 \mathrm{E}-2$ & $1.455 \mathrm{E}-5$ & $7.207 \mathrm{E}-2$ & $4.896 \mathrm{E}-6$ & 8.311E-1 & $2.338 \mathrm{E}-3$ & $7.240 \mathrm{E}-2$ & $4.369 \mathrm{E}-5$ \\
\hline 20 & $2.064 \mathrm{E}-2$ & $2.910 \mathrm{E}-5$ & $7.715 \mathrm{E}-2$ & 3.025E-5 & 7.496E-1 & $3.338 \mathrm{E}-3$ & $1.484 \mathrm{E}-1$ & $1.672 \mathrm{E}-4$ \\
\hline 30 & $1.864 \mathrm{E}-2$ & $4.285 \mathrm{E}-5$ & $8.187 \mathrm{E}-2$ & $9.349 \mathrm{E}-5$ & $6.719 \mathrm{E}-1$ & $3.911 \mathrm{E}-3$ & $2.217 \mathrm{E}-1$ & $3.813 \mathrm{E}-4$ \\
\hline 40 & $1.593 \mathrm{E}-2$ & $5.504 \mathrm{E}-5$ & 8.632E-2 & $2.061 \mathrm{E}-4$ & $6.125 \mathrm{E}-1$ & $4.096 \mathrm{E}-3$ & $2.773 \mathrm{E}-1$ & $6.763 \mathrm{E}-4$ \\
\hline 50 & $1.275 \mathrm{E}-2$ & $6.490 \mathrm{E}-5$ & $9.038 \mathrm{E}-2$ & $3.629 \mathrm{E}-4$ & $5.788 \mathrm{E}-1$ & 3.993E-3 & 3.077E-1 & $1.022 \mathrm{E}-3$ \\
\hline 60 & $9.541 \mathrm{E}-3$ & $7.193 \mathrm{E}-5$ & $9.391 \mathrm{E}-2$ & $5.401 \mathrm{E}-4$ & $5.678 \mathrm{E}-1$ & $3.736 \mathrm{E}-3$ & $3.154 \mathrm{E}-1$ & $1.376 \mathrm{E}-3$ \\
\hline \multicolumn{9}{|c|}{ Pin 72 , State 2} \\
\hline 0 & $2.284 \mathrm{E}-2$ & 0.0 & $9.980 \mathrm{E}-2$ & 0.0 & $8.644 \mathrm{E}-1$ & $1.390 \mathrm{E}-3$ & $1.160 \mathrm{E}-2$ & 0.0 \\
\hline 10 & $2.205 \mathrm{E}-2$ & $2.160 \mathrm{E}-5$ & $1.114 \mathrm{E}-1$ & $6.902 \mathrm{E}-6$ & $7.921 \mathrm{E}-1$ & $3.632 \mathrm{E}-3$ & $7.053 \mathrm{E}-2$ & $6.819 \mathrm{E}-5$ \\
\hline 20 & $2.053 \mathrm{E}-2$ & 4.364E-5 & $1.203 \mathrm{E}-1$ & $4.248 \mathrm{E}-5$ & $7.100 \mathrm{E}-1$ & $5.234 \mathrm{E}-3$ & $1.429 \mathrm{E}-1$ & $2.632 \mathrm{E}-4$ \\
\hline 30 & $1.831 \mathrm{E}-2$ & $6.494 \mathrm{E}-5$ & $1.289 \mathrm{E}-1$ & $1.308 \mathrm{E}-4$ & $6.330 \mathrm{E}-1$ & $6.191 \mathrm{E}-3$ & $2.112 E-1$ & $6.061 \mathrm{E}-4$ \\
\hline 40 & $1.547 \mathrm{E}-2$ & $8.427 \mathrm{E}-5$ & $1.372 \mathrm{E}-1$ & $2.871 \mathrm{E}-4$ & $5.745 \mathrm{E}-1$ & $6.545 \mathrm{E}-3$ & 2.619E-1 & $1.086 \mathrm{E}-3$ \\
\hline 50 & $1.227 \mathrm{E}-2$ & $1.003 \mathrm{E}-4$ & $1.449 \mathrm{E}-1$ & $5.033 \mathrm{E}-4$ & 5.407E-1 & $6.434 \mathrm{E}-3$ & $2.885 \mathrm{E}-1$ & $1.654 \mathrm{E}-3$ \\
\hline 60 & $9.108 \mathrm{E}-3$ & $1.120 \mathrm{E}-4$ & $1.518 \mathrm{E}-1$ & $7.462 \mathrm{E}-4$ & $5.284 \mathrm{E}-1$ & $6.064 E-3$ & $2.938 \mathrm{E}-1$ & $2.245 \mathrm{E}-3$ \\
\hline
\end{tabular}


Table 4.24. Fuel-averaged microscopic production cross section for Variant 11, States 1 and 2

\begin{tabular}{|c|c|c|c|c|c|c|c|c|}
\hline \multirow{2}{*}{$\begin{array}{c}\text { Burnup } \\
\text { (MWd/kg) }\end{array}$} & \multicolumn{8}{|c|}{ Microscopic production cross section (b) } \\
\hline & ${ }^{235} \mathrm{U}$ & ${ }^{236} \mathrm{U}$ & ${ }^{238} \mathrm{U}$ & ${ }^{238} \mathrm{Pu}$ & ${ }^{239} \mathrm{Pu}$ & ${ }^{240} \mathrm{Pu}$ & ${ }^{41} \mathrm{Pu}$ & ${ }^{2} \mathrm{Pu}$ \\
\hline \multicolumn{9}{|c|}{ Pin 1, State 1} \\
\hline 0 & $1.007 \mathrm{E}+2$ & 7.712E-1 & $2.862 \mathrm{E}-1$ & $158 \mathrm{E}+0$ & $3.409 E+2$ & $1.823 E+0$ & $3.610 \mathrm{E}+2$ & $1.328 \mathrm{E}+0$ \\
\hline 10 & $9.464 \mathrm{E}+1$ & $7.903 \mathrm{E}-1$ & $2.930 \mathrm{E}-1$ & $6.856 \mathrm{E}+0$ & $2.827 \mathrm{E}+2$ & $1.894 \mathrm{E}+0$ & $3.057 \mathrm{E}+2$ & $1.413 \mathrm{E}+0$ \\
\hline 20 & $9.365 E+1$ & 7.798E-1 & $2.960 \mathrm{E}-1$ & $6.872 \mathrm{E}+0$ & $2.715 \mathrm{E}+2$ & $1.884 \mathrm{E}+0$ & $2.982 E+2$ & $1.423 \mathrm{E}+0$ \\
\hline 30 & $9.497 \mathrm{E}+1$ & 7.720E-1 & $2.972 \mathrm{E}-1$ & $6.929 E+0$ & $2.702 E+2$ & $1.876 \mathrm{E}+0$ & $2.997 E+2$ & $1.425 \mathrm{E}+0$ \\
\hline 40 & $9.732 \mathrm{E}+1$ & 7.663E-1 & $2.976 \mathrm{E}-1$ & $7.004 \mathrm{E}+0$ & $2.733 E+2$ & $1.869 \mathrm{E}+0$ & $3.051 E+2$ & $1.425 \mathrm{E}+0$ \\
\hline 50 & $9.992 \mathrm{E}+1$ & 7.625E-1 & $2.978 \mathrm{E}-1$ & $7.081 E+0$ & $2.781 \mathrm{E}+2$ & $1.863 \mathrm{E}+0$ & $3.119 \mathrm{E}+2$ & $1.423 \mathrm{E}+0$ \\
\hline 60 & $1.022 E+2$ & $7.605 \mathrm{E}-1$ & $2.980 \mathrm{E}-1$ & $7.148 \mathrm{E}+0$ & $2.827 E+2$ & $1.860 \mathrm{E}+0$ & $3.181 E+2$ & $1.422 \mathrm{E}+0$ \\
\hline \multicolumn{9}{|c|}{ Pin 1, State } \\
\hline 0 & $9.256 \mathrm{E}+1$ & $7.854 \mathrm{E}-1$ & $2.999 \mathrm{E}-1$ & $6.983 E+0$ & $3.128 E+2$ & $1.877 \mathrm{E}+0$ & 3.309E+2 & $1.379 \mathrm{E}+0$ \\
\hline 10 & 8.741E+1 & $8.088 \mathrm{E}-1$ & $3.062 \mathrm{E}-1$ & $6.745 \mathrm{E}+0$ & $2.605 E+2$ & $1.959 \mathrm{E}+0$ & $2.815 \mathrm{E}+2$ & $1.472 \mathrm{E}+0$ \\
\hline 20 & $8.655 \mathrm{E}+1$ & $7.986 \mathrm{E}-1$ & $3.091 \mathrm{E}-1$ & $6.761 E+0$ & $2.505 E+2$ & $1.951 E+0$ & $2.749 \mathrm{E}+2$ & $1.481 \mathrm{E}+0$ \\
\hline 30 & $8.768 E+1$ & $7.912 \mathrm{E}-1$ & $3.104 \mathrm{E}-1$ & $6.811 E+0$ & $2.491 E+2$ & $1.943 E+0$ & $2.760 \mathrm{E}+2$ & $1.484 \mathrm{E}+0$ \\
\hline 40 & $8.967 \mathrm{E}+1$ & $7.860 \mathrm{E}-1$ & $3.109 \mathrm{E}-1$ & $6.876 \mathrm{E}+0$ & $2.516 \mathrm{E}+2$ & $1.937 \mathrm{E}+0$ & $.805 E+2$ & $.484 E+0$ \\
\hline 50 & $9.186 \mathrm{E}+1$ & $7.826 \mathrm{E}-1$ & $3.112 \mathrm{E}-1$ & $6.941 E+0$ & $2.555 E+2$ & $1.932 \mathrm{E}+0$ & $861 E+2$ & $483 E+0$ \\
\hline 60 & $9.374 \mathrm{E}+1$ & $7.811 \mathrm{E}-1$ & $116 \mathrm{E}-1$ & $6.997 \mathrm{E}+0$ & $2.592 \mathrm{E}+2$ & $1.930 \mathrm{E}+0$ & $911 \mathrm{E}+2$ & $483 E+0$ \\
\hline \multicolumn{9}{|c|}{ Pin 29, State 1} \\
\hline 0 & $9.131 \mathrm{E}+1$ & $7.757 \mathrm{E}-1$ & $2.898 \mathrm{E}-1$ & $6.902 \mathrm{E}+0$ & $3.141 E+2$ & $1.856 \mathrm{E}+0$ & $292 E+2$ & $.358 \mathrm{E}+0$ \\
\hline 10 & $8.513 \mathrm{E}+1$ & $7.913 \mathrm{E}-1$ & $2.969 \mathrm{E}-1$ & $6.628 \mathrm{E}+0$ & $2.566 \mathrm{E}+2$ & $1.923 E+0$ & $2.757 \mathrm{E}+2$ & $1.440 \mathrm{E}+0$ \\
\hline 20 & $8.379 \mathrm{E}+1$ & $7.810 \mathrm{E}-1$ & $3.006 \mathrm{E}-1$ & $6.640 \mathrm{E}+0$ & $2.440 \mathrm{E}+2$ & $1.916 \mathrm{E}+0$ & $2.671 E+2$ & $1.452 E+0$ \\
\hline 30 & $74 E+1$ & $7.735 \mathrm{E}-1$ & 024E-1 & $6.691 \mathrm{E}+0$ & $2.414 \mathrm{E}+2$ & $1.910 \mathrm{E}+0$ & $2.673 \mathrm{E}+2$ & $1.458 \mathrm{E}+0$ \\
\hline 40 & $679 E+1$ & $7.681 \mathrm{E}-1$ & $3.034 E-1$ & $6.761 \mathrm{E}+0$ & $2.436 \mathrm{E}+2$ & $905 \mathrm{E}+0$ & $2.719 \mathrm{E}+2$ & $1.459 \mathrm{E}+0$ \\
\hline 50 & $925 \mathrm{E}+1$ & 7.642E-1 & $3.039 \mathrm{E}-1$ & $6.836 \mathrm{E}+0$ & $2.479 \mathrm{E}+2$ & $1.901 E+0$ & $2.782 E+2$ & $459 E+0$ \\
\hline 60 & $56 \mathrm{E}+1$ & $7.619 \mathrm{E}-1$ & 043E-1 & $6.904 \mathrm{E}+0$ & $2.525 E+2$ & $1.897 \mathrm{E}+0$ & $.844 \mathrm{E}+2$ & $.459 \mathrm{E}+0$ \\
\hline \multicolumn{9}{|c|}{ Pin 29, State 2} \\
\hline 0 & $E+1$ & $7.855 \mathrm{E}-1$ & 3.011E-1 & $6.554 \mathrm{E}+0$ & $2.601 E+2$ & $1.911 E+0$ & $2.721 E+2$ & $.413 E+0$ \\
\hline 10 & $2 \mathrm{E}+1$ & $8.020 \mathrm{E}-1$ & $75 \mathrm{E}-1$ & $6.360 \mathrm{E}+0$ & $2.128 \mathrm{E}+2$ & $1.983 E+0$ & $2.284 \mathrm{E}+2$ & $1.496 \mathrm{E}+0$ \\
\hline 20 & $8 \mathrm{E}+1$ & $7.923 \mathrm{E}-1$ & $3.110 \mathrm{E}-1$ & $6.370 \mathrm{E}+0$ & $2.024 \mathrm{E}+2$ & $1.977 \mathrm{E}+0$ & $2.213 E+2$ & $1.508 \mathrm{E}+0$ \\
\hline 30 & $59 \mathrm{E}+1$ & $7.858 \mathrm{E}-1$ & $3.129 \mathrm{E}-1$ & $6.408 \mathrm{E}+0$ & $1.998 \mathrm{E}+2$ & $1.973 E+0$ & $2.210 \mathrm{E}+2$ & $513 E+0$ \\
\hline 40 & $203 E+1$ & $7.812 \mathrm{E}-1$ & 3.141E-1 & $6.460 \mathrm{E}+0$ & $2.010 \mathrm{E}+2$ & $1.970 \mathrm{E}+0$ & $2.239 \mathrm{E}+2$ & $516 \mathrm{E}+0$ \\
\hline 50 & $7.376 \mathrm{E}+1$ & $7.783 \mathrm{E}-1$ & $.149 \mathrm{E}-1$ & $6.515 \mathrm{E}+0$ & $2.039 \mathrm{E}+2$ & $1.967 \mathrm{E}+0$ & $2.283 \mathrm{E}+2$ & $517 E+0$ \\
\hline 60 & $7.535 \mathrm{E}+1$ & $7.766 \mathrm{E}-1$ & 155E-1 & $564 \mathrm{E}+0$ & $2.069 E+2$ & $1.966 \mathrm{E}+0$ & $2.324 E+2$ & $1.517 \mathrm{E}+0$ \\
\hline \multicolumn{9}{|c|}{ Pin 72, State 1} \\
\hline 0 & $70 \mathrm{E}+1$ & $722 \mathrm{E}-1$ & 1E-1 & $7.043 E+0$ & $3.293 E+2$ & $1.837 \mathrm{E}+0$ & $3.472 \mathrm{E}+2$ & $.341 E+0$ \\
\hline 10 & $E+1$ & $7.893 \mathrm{E}-1$ & 41E-1 & $6.759 E+0$ & $2.718 E+2$ & $1.905 \mathrm{E}+0$ & $2.932 E+2$ & $1.424 \mathrm{E}+0$ \\
\hline 20 & $E+1$ & $7.788 \mathrm{E}-1$ & 73E-1 & $6.776 \mathrm{E}+0$ & $2.603 E+2$ & $1.897 \mathrm{E}+0$ & $2.856 E+2$ & $1.435 \mathrm{E}+0$ \\
\hline 30 & $95 \mathrm{E}+1$ & 7.709E-1 & 7E-1 & $3 \mathrm{E}+0$ & $3 E+2$ & $1.889 \mathrm{E}+0$ & $2.869 \mathrm{E}+2$ & $1.438 E+0$ \\
\hline 40 & $32 E+1$ & $7.652 \mathrm{E}-1$ & $93 \mathrm{E}-1$ & $6.909 E+0$ & $2.619 E+2$ & $1.882 \mathrm{E}+0$ & $2.924 \mathrm{E}+2$ & $438 \mathrm{E}+0$ \\
\hline 50 & $01 \mathrm{E}+1$ & $7.613 \mathrm{E}-1$ & 2.995E-1 & $6.989 \mathrm{E}+0$ & $2.669 E+2$ & $1.876 \mathrm{E}+0$ & +2 & $37 \mathrm{E}+0$ \\
\hline 60 & $9.842 E+1$ & $7.590 \mathrm{E}-1$ & $2.997 \mathrm{E}-1$ & $7.059 E+0$ & $2.718 \mathrm{E}+2$ & $1.873 \mathrm{E}+0$ & $3.060 \mathrm{E}+2$ & $1.436 \mathrm{E}+0$ \\
\hline \multicolumn{9}{|c|}{ Pin 72, State } \\
\hline 0 & $8 \mathrm{E}+1$ & $7.855 \mathrm{E}-1$ & E-1 & $6.183 E+0$ & $2.029 E+2$ & $1.939 \mathrm{E}+0$ & $2.122 E+2$ & $1.445 \mathrm{E}+0$ \\
\hline 10 & $34 \mathrm{E}+1$ & $7.952 \mathrm{E}-1$ & $3.093 \mathrm{E}-1$ & $6.037 \mathrm{E}+0$ & $1.659 E+2$ & $1.994 \mathrm{E}+0$ & $1.784 \mathrm{E}+2$ & $1.513 E+0$ \\
\hline 20 & $57 \mathrm{E}+1$ & 7.857E-1 & 3.122E-1 & $6.048 \mathrm{E}+0$ & $1.582 \mathrm{E}+2$ & $1.989 \mathrm{E}+0$ & $1.733 E+2$ & $1.522 \mathrm{E}+0$ \\
\hline 30 & $07 E+1$ & $7.795 \mathrm{E}-1$ & 3.137E-1 & $6.078 \mathrm{E}+0$ & $1.565 E+2$ & $1.985 \mathrm{E}+0$ & $1.732 E+2$ & $1.526 \mathrm{E}+0$ \\
\hline 40 & $12 E+1$ & $7.756 \mathrm{E}-1$ & 3.148E-1 & $6.116 \mathrm{E}+0$ & $1.574 \mathrm{E}+2$ & $1.982 \mathrm{E}+0$ & $1.754 \mathrm{E}+2$ & $1.528 \mathrm{E}+0$ \\
\hline 50 & $5.834 \mathrm{E}+1$ & 7.733E-1 & $3.155 \mathrm{E}-1$ & $6.155 E+0$ & $1.594 \mathrm{E}+2$ & $1.980 \mathrm{E}+0$ & $1.784 \mathrm{E}+2$ & $1.529 \mathrm{E}+0$ \\
\hline 60 & $5.940 \mathrm{E}+1$ & $7.725 \mathrm{E}-1$ & $3.163 \mathrm{E}-1$ & $6.188 \mathrm{E}+0$ & $1.615 \mathrm{E}+2$ & $1.980 \mathrm{E}+0$ & $1.812 \mathrm{E}+2$ & $1.530 \mathrm{E}+0$ \\
\hline
\end{tabular}


Table 4.25. Fuel-averaged microscopic production cross section for Variant 12, States 1 and 2

\begin{tabular}{|c|c|c|c|c|c|c|c|c|}
\hline \multirow{2}{*}{$\begin{array}{c}\text { Burnup } \\
\text { (MWd/kg) }\end{array}$} & \multicolumn{8}{|c|}{ Microscopic production cross section (b) } \\
\hline & ${ }^{235} \mathrm{U}$ & ${ }^{236} \mathrm{U}$ & ${ }^{238} \mathrm{U}$ & ${ }^{238} \mathrm{Pu}$ & ${ }^{239} \mathrm{Pu}$ & ${ }^{240} \mathrm{Pu}$ & ${ }^{24} \mathrm{Pu}$ & ${ }^{242} \mathrm{Pu}$ \\
\hline \multicolumn{9}{|c|}{ Pin 1, State 1} \\
\hline 0 & $5.831 E+1$ & $8.456 \mathrm{E}-1$ & $3.232 \mathrm{E}-1$ & $6.266 \mathrm{E}+0$ & $1.384 \mathrm{E}+2$ & $2.001 \mathrm{E}+0$ & $1.685 \mathrm{E}+2$ & $1.465 \mathrm{E}+0$ \\
\hline 10 & $6.173 E+1$ & 8.457E-1 & $3.208 \mathrm{E}-1$ & $6.216 \mathrm{E}+0$ & $1.529 \mathrm{E}+2$ & $1.968 \mathrm{E}+0$ & $1.830 \mathrm{E}+2$ & $1.523 \mathrm{E}+0$ \\
\hline 20 & $6.731 E+1$ & 8.284E-1 & $3.169 \mathrm{E}-1$ & $6.349 E+0$ & $1.727 \mathrm{E}+2$ & $1.943 E+0$ & $2.020 \mathrm{E}+2$ & $1.507 \mathrm{E}+0$ \\
\hline 30 & $7.331 E+1$ & 8.137E-1 & $3.129 \mathrm{E}-1$ & $6.487 \mathrm{E}+0$ & $1.933 E+2$ & $1.921 E+0$ & $2.221 E+2$ & $1.490 \mathrm{E}+0$ \\
\hline 40 & $7.922 \mathrm{E}+1$ & $8.020 \mathrm{E}-1$ & $3.094 \mathrm{E}-1$ & $6.624 E+0$ & $2.128 \mathrm{E}+2$ & $1.903 E+0$ & $2.417 \mathrm{E}+2$ & $1.475 \mathrm{E}+0$ \\
\hline 50 & $8.465 \mathrm{E}+1$ & $7.929 \mathrm{E}-1$ & 3.067E-1 & $6.752 \mathrm{E}+0$ & $2.297 \mathrm{E}+2$ & $1.890 \mathrm{E}+0$ & $2.595 \mathrm{E}+2$ & $1.463 \mathrm{E}+0$ \\
\hline 60 & $8.937 \mathrm{E}+1$ & $7.862 \mathrm{E}-1$ & $3.048 \mathrm{E}-1$ & $6.864 \mathrm{E}+0$ & $2.437 E+2$ & $1.881 E+0$ & $2.748 \mathrm{E}+2$ & $1.454 \mathrm{E}+0$ \\
\hline \multicolumn{9}{|c|}{ Pin 1, State 2} \\
\hline 0 & $5.514 E+1$ & $8.539 \mathrm{E}-1$ & $3.346 \mathrm{E}-1$ & $6.209 E+0$ & $1.303 E+2$ & $2.057 \mathrm{E}+0$ & $1.589 \mathrm{E}+2$ & $1.504 \mathrm{E}+0$ \\
\hline 10 & $5.833 \mathrm{E}+1$ & 8.593E-1 & 3.321E-1 & $6.186 \mathrm{E}+0$ & $1.439 \mathrm{E}+2$ & $2.025 \mathrm{E}+0$ & $1.724 E+2$ & $1.573 \mathrm{E}+0$ \\
\hline 20 & $6.339 \mathrm{E}+1$ & $8.428 \mathrm{E}-1$ & $3.283 \mathrm{E}-1$ & $6.306 \mathrm{E}+0$ & $1.621 \mathrm{E}+2$ & $2.002 E+0$ & $1.898 \mathrm{E}+2$ & $1.557 E+0$ \\
\hline 30 & $6.877 \mathrm{E}+1$ & $8.290 \mathrm{E}-1$ & $3.245 \mathrm{E}-1$ & $6.429 \mathrm{E}+0$ & $1.808 \mathrm{E}+2$ & $1.981 E+0$ & $2.078 E+2$ & $1.541 E+0$ \\
\hline 40 & $7.400 \mathrm{E}+1$ & $8.181 E-1$ & $3.213 \mathrm{E}-1$ & $6.549 \mathrm{E}+0$ & $1.983 E+2$ & $1.965 \mathrm{E}+0$ & $2.252 \mathrm{E}+2$ & $1.528 \mathrm{E}+0$ \\
\hline 50 & $7.875 \mathrm{E}+1$ & $8.100 \mathrm{E}-1$ & $3.190 \mathrm{E}-1$ & $6.660 \mathrm{E}+0$ & $2.133 E+2$ & $1.953 \mathrm{E}+0$ & $2.408 \mathrm{E}+2$ & $1.517 \mathrm{E}+0$ \\
\hline 60 & $8.281 E+1$ & $8.041 \mathrm{E}-1$ & 3.174E-1 & $6.757 \mathrm{E}+0$ & $2.255 E+2$ & $1.946 \mathrm{E}+0$ & $2.540 \mathrm{E}+2$ & $1.510 \mathrm{E}+0$ \\
\hline \multicolumn{9}{|c|}{ Pin 29, State 1} \\
\hline 0 & $5.086 \mathrm{E}+1$ & $8.478 \mathrm{E}-1$ & $3.241 \mathrm{E}-1$ & $6.061 \mathrm{E}+0$ & $1.173 E+2$ & $2.013 E+0$ & $1.449 \mathrm{E}+2$ & $1.488 \mathrm{E}+0$ \\
\hline 10 & $5.322 \mathrm{E}+1$ & $8.471 \mathrm{E}-1$ & $3.236 \mathrm{E}-1$ & $6.018 \mathrm{E}+0$ & $1.276 \mathrm{E}+2$ & $1.992 \mathrm{E}+0$ & $1.552 \mathrm{E}+2$ & $1.546 \mathrm{E}+0$ \\
\hline 20 & $5.766 \mathrm{E}+1$ & 8.327E-1 & $3.215 \mathrm{E}-1$ & $6.131 E+0$ & $1.431 \mathrm{E}+2$ & $1.976 \mathrm{E}+0$ & $1.704 E+2$ & $1.536 \mathrm{E}+0$ \\
\hline 30 & $6.278 \mathrm{E}+1$ & $8.200 \mathrm{E}-1$ & $3.189 \mathrm{E}-1$ & $6.254 \mathrm{E}+0$ & $1.606 \mathrm{E}+2$ & $1.960 \mathrm{E}+0$ & $1.876 \mathrm{E}+2$ & $1.525 \mathrm{E}+0$ \\
\hline 40 & $6.818 \mathrm{E}+1$ & 8.091E-1 & 3.162E-1 & $6.380 \mathrm{E}+0$ & $1.786 \mathrm{E}+2$ & $1.945 \mathrm{E}+0$ & $2.056 E+2$ & $1.513 E+0$ \\
\hline 50 & $7.341 \mathrm{E}+1$ & 8.001E-1 & $3.138 \mathrm{E}-1$ & $6.503 E+0$ & $1.954 \mathrm{E}+2$ & $1.932 \mathrm{E}+0$ & $2.229 \mathrm{E}+2$ & $1.502 E+0$ \\
\hline 60 & $7.814 \mathrm{E}+1$ & $7.929 \mathrm{E}-1$ & $3.119 \mathrm{E}-1$ & $6.615 E+0$ & $2.101 E+2$ & $1.922 \mathrm{E}+0$ & $2.385 E+2$ & $1.493 E+0$ \\
\hline \multicolumn{9}{|c|}{ Pin 29, State 2} \\
\hline 0 & $4.452 \mathrm{E}+1$ & $8.459 \mathrm{E}-1$ & 2E-1 & $5.922 \mathrm{E}+0$ & $1.010 \mathrm{E}+2$ & $2.056 \mathrm{E}+0$ & $1.256 \mathrm{E}+2$ & $1.523 E+0$ \\
\hline 10 & $4.636 E+1$ & $8.485 \mathrm{E}-1$ & $3.308 \mathrm{E}-1$ & $5.901 E+0$ & $1.094 E+2$ & $2.037 \mathrm{E}+0$ & $1.338 \mathrm{E}+2$ & $1.585 \mathrm{E}+0$ \\
\hline 20 & $4.979 \mathrm{E}+1$ & $8.360 \mathrm{E}-1$ & $3.290 \mathrm{E}-1$ & $5.988 \mathrm{E}+0$ & $1.219 E+2$ & $2.024 \mathrm{E}+0$ & $1.457 \mathrm{E}+2$ & $1.578 \mathrm{E}+0$ \\
\hline 30 & $5.372 \mathrm{E}+1$ & $8.251 \mathrm{E}-1$ & $3.269 E-1$ & $6.082 E+0$ & $1.358 E+2$ & $2.011 E+0$ & $1.590 \mathrm{E}+2$ & $1.569 \mathrm{E}+0$ \\
\hline 40 & $5.782 \mathrm{E}+1$ & 8.159E-1 & $3.247 \mathrm{E}-1$ & $6.178 E+0$ & $1.499 E+2$ & $1.999 \mathrm{E}+0$ & $1.728 \mathrm{E}+2$ & $1.559 \mathrm{E}+0$ \\
\hline 50 & $6.175 E+1$ & $8.086 \mathrm{E}-1$ & $3.229 \mathrm{E}-1$ & $6.269 \mathrm{E}+0$ & $1.630 \mathrm{E}+2$ & $1.990 \mathrm{E}+0$ & $1.859 \mathrm{E}+2$ & $1.551 \mathrm{E}+0$ \\
\hline 60 & $6.528 \mathrm{E}+1$ & $8.028 \mathrm{E}-1$ & $3.215 \mathrm{E}-1$ & $6.352 \mathrm{E}+0$ & $1.742 \mathrm{E}+2$ & $1.982 \mathrm{E}+0$ & $1.976 \mathrm{E}+2$ & $1.544 \mathrm{E}+0$ \\
\hline \multicolumn{9}{|c|}{ Pin 72, State 1} \\
\hline 0 & $5.497 \mathrm{E}+1$ & $8.458 \mathrm{E}-1$ & $3.230 \mathrm{E}-1$ & $6.170 \mathrm{E}+0$ & $1.289 E+2$ & $2.005 E+0$ & $1.579 \mathrm{E}+2$ & $1.476 \mathrm{E}+0$ \\
\hline 10 & $5.801 E+1$ & $8.451 \mathrm{E}-1$ & $3.214 \mathrm{E}-1$ & $6.127 \mathrm{E}+0$ & $1.418 \mathrm{E}+2$ & $1.977 \mathrm{E}+0$ & $1.707 \mathrm{E}+2$ & $1.533 E+0$ \\
\hline 20 & $6.322 E+1$ & $8.289 \mathrm{E}-1$ & $3.182 E-1$ & $6.254 \mathrm{E}+0$ & $1.600 \mathrm{E}+2$ & $1.956 \mathrm{E}+0$ & $1.886 \mathrm{E}+2$ & $1.519 \mathrm{E}+0$ \\
\hline 30 & $6.904 \mathrm{E}+1$ & $8.149 E-1$ & $3.147 \mathrm{E}-1$ & $6.391 E+0$ & $1.799 E+2$ & $1.936 \mathrm{E}+0$ & $2.081 E+2$ & $1.504 \mathrm{E}+0$ \\
\hline 40 & $7.496 \mathrm{E}+1$ & 8.033E-1 & $3.115 \mathrm{E}-1$ & $6.528 \mathrm{E}+0$ & $1.994 \mathrm{E}+2$ & $1.919 E+0$ & $2.277 \mathrm{E}+2$ & $1.490 \mathrm{E}+0$ \\
\hline 50 & $8.051 E+1$ & $7.940 \mathrm{E}-1$ & $3.088 \mathrm{E}-1$ & $6.658 \mathrm{E}+0$ & $2.169 \mathrm{E}+2$ & $1.905 \mathrm{E}+0$ & $2.460 \mathrm{E}+2$ & $1.477 \mathrm{E}+0$ \\
\hline 60 & $8.540 \mathrm{E}+1$ & $7.869 \mathrm{E}-1$ & $3.068 \mathrm{E}-1$ & $6.774 \mathrm{E}+0$ & $2.316 \mathrm{E}+2$ & $1.895 E+0$ & $2.619 \mathrm{E}+2$ & $1.468 \mathrm{E}+0$ \\
\hline \multicolumn{9}{|c|}{ Pin 72, State 2} \\
\hline 0 & $3.730 E+1$ & $8.352 \mathrm{E}-1$ & $3.307 \mathrm{E}-1$ & $5.754 \mathrm{E}+0$ & $8.226 \mathrm{E}+1$ & $2.058 \mathrm{E}+0$ & $1.035 \mathrm{E}+2$ & $1.539 E+0$ \\
\hline 10 & $3.883 \mathrm{E}+1$ & 8.325E-1 & $3.296 \mathrm{E}-1$ & $5.733 E+0$ & $8.968 \mathrm{E}+1$ & $2.039 E+0$ & $1.104 \mathrm{E}+2$ & $1.588 \mathrm{E}+0$ \\
\hline 20 & $4.151 \mathrm{E}+1$ & $8.203 E-1$ & $3.274 \mathrm{E}-1$ & $5.797 E+0$ & $1.001 E+2$ & $2.024 E+0$ & $1.198 \mathrm{E}+2$ & $1.578 \mathrm{E}+0$ \\
\hline 30 & $4.450 \mathrm{E}+1$ & $8.103 E-1$ & $3.251 \mathrm{E}-1$ & $5.865 \mathrm{E}+0$ & $1.112 \mathrm{E}+2$ & $2.011 \mathrm{E}+0$ & $1.301 E+2$ & $1.569 \mathrm{E}+0$ \\
\hline 40 & $4.750 E+1$ & 8.023E-1 & $3.230 \mathrm{E}-1$ & $5.932 \mathrm{E}+0$ & $1.220 \mathrm{E}+2$ & $2.000 \mathrm{E}+0$ & $1.403 E+2$ & $1.560 \mathrm{E}+0$ \\
\hline 50 & $5.029 \mathrm{E}+1$ & $7.963 \mathrm{E}-1$ & $3.214 \mathrm{E}-1$ & $5.995 E+0$ & $1.315 E+2$ & $1.993 E+0$ & $1.497 \mathrm{E}+2$ & $1.553 \mathrm{E}+0$ \\
\hline 60 & $270 \mathrm{E}+1$ & $7.921 \mathrm{E}-1$ & $3.205 \mathrm{E}-1$ & $6.050 \mathrm{E}+0$ & $1.393 E+2$ & $1.988 \mathrm{E}+0$ & $1.577 \mathrm{E}+2$ & $1.548 \mathrm{E}+0$ \\
\hline
\end{tabular}


Table 4.26. Fuel-averaged microscopic absorption cross sections for uranium and plutonium isotopes vs burnup for Variants $1-4$ and 1-10, State 1

\begin{tabular}{|c|c|c|c|c|c|c|c|c|c|c|}
\hline \multirow{2}{*}{$\begin{array}{c}\text { Burnup } \\
\text { (MWd/kg) }\end{array}$} & \multicolumn{10}{|c|}{ Microscopic absorption cross section (b) } \\
\hline & ${ }^{235} \mathrm{U}$ & ${ }^{236} \mathrm{U}$ & ${ }^{238} \mathrm{U}$ & ${ }^{238} \mathrm{Pu}$ & ${ }^{239} \mathrm{Pu}$ & ${ }^{241} \mathrm{Pu}$ & ${ }^{242} \mathrm{Pu}$ & ${ }^{135} \mathrm{Xe}$ & ${ }^{149} \mathrm{Sm}$ & Total FP \\
\hline \multicolumn{11}{|c|}{ Variant I } \\
\hline 0 & $4.292 \mathrm{E}+1$ & $8.621 \mathrm{E}+0$ & $9.693 \mathrm{E}-1$ & $2.729 \mathrm{E}+1$ & $1.491 \mathrm{E}+2 \quad 2.242 \mathrm{E}+2$ & $1.272 \mathrm{E}+2$ & $3.352 E+1$ & $1.429 \mathrm{E}+5$ & $4.526 \mathrm{E}+3$ & $2.027 \mathrm{E}+4$ \\
\hline 10 & $3.919 \mathrm{E}+1$ & $7.517 \mathrm{E}+0$ & $9.681 \mathrm{E}-1$ & $2.499 \mathrm{E}+1$ & $1.267 \mathrm{E}+2 \quad 1.719 \mathrm{E}+2$ & $1.123 \mathrm{E}+2$ & $3.354 \mathrm{E}+1$ & $1.275 \mathrm{E}+5$ & $4.026 \mathrm{E}+3$ & $9.960 \mathrm{E}+0$ \\
\hline 20 & $3.789 \mathrm{E}+1$ & $6.931 \mathrm{E}+0$ & 9.705E-1 & $2.426 \mathrm{E}+1$ & $1.175 \mathrm{E}+2 \quad 1.311 \mathrm{E}+2$ & $1.062 \mathrm{E}+2$ & $3.302 \mathrm{E}+1$ & $1.227 \mathrm{E}+5$ & $3.856 \mathrm{E}+3$ & $6.930 \mathrm{E}+0$ \\
\hline 30 & $3.759 \mathrm{E}+1$ & $6.569 \mathrm{E}+0$ & $9.743 \mathrm{E}-1$ & $2.416 \mathrm{E}+1$ & $1.136 \mathrm{E}+2 \quad 1.093 \mathrm{E}+2$ & $1.041 \mathrm{E}+2$ & $3.185 \mathrm{E}+1$ & $1.220 \mathrm{E}+5$ & $3.821 \mathrm{E}+3$ & $5.805 \mathrm{E}+0$ \\
\hline 40 & $3.771 E+1$ & $6.355 \mathrm{E}+0$ & $9.785 \mathrm{E}-1$ & $2.432 E+1$ & $1.121 \mathrm{E}+29.684 \mathrm{E}+1$ & $1.035 \mathrm{E}+2$ & $3.028 \mathrm{E}+1$ & $1.232 \mathrm{E}+5$ & $3.842 \mathrm{E}+3$ & $5.171 \mathrm{E}+0$ \\
\hline 50 & $3.796 \mathrm{E}+1$ & $6.212 \mathrm{E}+0$ & $9.823 \mathrm{E}-1$ & $2.455 \mathrm{E}+1$ & $1.116 \mathrm{E}+28.901 \mathrm{E}+1$ & & $2.860 \mathrm{E}+1$ & $1.248 \mathrm{E}+5$ & $3.882 \mathrm{E}+3$ & $4.727 E+0$ \\
\hline 60 & $3.819 \mathrm{E}+1$ & $6.131 E+0$ & $9.857 \mathrm{E}-1$ & $2.475 \mathrm{E}+1$ & $1.114 \mathrm{E}+28.390 \mathrm{E}+1$ & $1.039 \mathrm{E}+2$ & $2.696 \mathrm{E}+1$ & $1.263 \mathrm{E}+5$ & $3.919 E+3$ & $4.380 \mathrm{E}+0$ \\
\hline \multicolumn{11}{|c|}{ Variant 2} \\
\hline $\begin{array}{r}0 \\
10\end{array}$ & $\begin{array}{l}2.531 \mathrm{E}+1 \\
2.593 \mathrm{E}+1\end{array}$ & $\begin{array}{l}9.045 E+0 \\
8.691 E+0\end{array}$ & $\begin{array}{l}9.403 \mathrm{E}-1 \\
9.512 \mathrm{E}-1\end{array}$ & $\begin{array}{l}1.54 / \mathrm{E}+1 \\
1.600 \mathrm{E}+1\end{array}$ & $\begin{array}{l}5.906 \mathrm{E}+1 \\
6.323 \mathrm{E}+1 \quad 6.720 \mathrm{E}+1\end{array}$ & $\begin{array}{l}6.05 / \mathrm{E}+1 \\
6.367 \mathrm{E}+1\end{array}$ & $\begin{array}{l}3.529 E+1 \\
3.396 E+1\end{array}$ & $\begin{array}{l}6.262 E+4 \\
6.619 E+4\end{array}$ & $\begin{array}{l}2.003 E+3 \\
2.103 E+3\end{array}$ & $\begin{array}{l}8.900 E+3 \\
9.451 E+0\end{array}$ \\
\hline 20 & $2.727 E+1$ & $8.462 \mathrm{E}+0$ & $9.566 \mathrm{E}-1$ & $1.699 \mathrm{E}+1$ & $6.918 \mathrm{E}+1 \quad 5.844 \mathrm{E}+1$ & $6.820 \mathrm{E}+1$ & $3.162 \mathrm{E}+1$ & $7.297 \mathrm{E}+4$ & $2.305 \mathrm{E}+3$ & $6.901 E+0$ \\
\hline 30 & $2.870 \mathrm{E}+1$ & $8.258 \mathrm{E}+0$ & $9.620 \mathrm{E}-1$ & $1.801 \mathrm{E}+1$ & $7.530 \mathrm{E}+1 \quad 5.554 \mathrm{E}+1$ & $7.290 \mathrm{E}+1$ & $2.888 \mathrm{E}+1$ & $8.004 E+4$ & $2.516 \mathrm{E}+3$ & $5.900 \mathrm{E}+0$ \\
\hline 40 & $3.009 \mathrm{E}+1$ & $8.132 \mathrm{E}+0$ & 9.672E-1 & $1.900 \mathrm{E}+1$ & $8.101 \mathrm{E}+15$ & $E+1$ & $2.624 \mathrm{E}+1$ & $8.692 E+4$ & $2.723 E+3$ & $5.312 \mathrm{E}+0$ \\
\hline 50 & $3.136 \mathrm{E}+1$ & $8.020 \mathrm{E}+0$ & $9.718 \mathrm{E}-1$ & $1.991 \mathrm{E}+1$ & $8.600 \mathrm{E}+15$. & $8.155 \mathrm{E}+1$ & $2.410 \mathrm{E}+1$ & $9.322 E+4$ & $2.912 \mathrm{E}+3$ & $4.884 \mathrm{E}+0$ \\
\hline & $3.247 \mathrm{E}+1$ & $7.926 \mathrm{E}+0$ & $9.760 \mathrm{E}-1$ & $2.070 \mathrm{E}+1$ & $9.016 \mathrm{E}+15.783 \mathrm{E}+1$ & $8.511 E+1$ & $2.244 \mathrm{E}+1$ & $9.875 E+4$ & $3.078 E+3$ & $4.537 E+0$ \\
\hline \multirow{3}{*}{0} & & & & & iriant 3 & & & & & \\
\hline & $3.952 \mathrm{E}+1$ & $6.993 E+0$ & $9.843 \mathrm{E}-1$ & 2. & $1112 \mathrm{E}+2$ & $1 E+2$ & $3.262 \mathrm{E}+1$ & $1.307 \mathrm{E}+5$ & $4.088 \mathrm{E}+3$ & $1.850 \mathrm{E}+4$ \\
\hline & \multicolumn{10}{|c|}{ Variant 4} \\
\hline 0 & $3.757 \mathrm{E}+1$ & $6.709 \mathrm{E}+0$ & $9.765 \mathrm{E}-1$ & $2.413 E+1$ & $1.124 \mathrm{E}+2 \quad 1.061 \mathrm{E}+2$ & $1.032 E+2$ & $3.177 \mathrm{E}+1$ & $1.218 E+5$ & $3.815 E+3$ & $1.925 \mathrm{E}+1$ \\
\hline & & & & & Vario & & & & & \\
\hline 0 & $2.717 \mathrm{E}+1$ & $9.006 \mathrm{E}+0$ & $9.485 \mathrm{E}-1$ & $1.653 \mathrm{E}+1$ & 6.422 & & $3.518 \mathrm{E}+1$ & $6.964 \mathrm{E}$ & E- & +3 \\
\hline & & & & & Var & & & & & \\
\hline 0 & $4.570 \mathrm{E}+1$ & $8.754 \mathrm{E}+0$ & $9.836 \mathrm{E}-1$ & $\Xi+1$ & $1.561 \mathrm{E}$ & & 3.42 & & $\mathrm{E}$ & $2.240 \mathrm{E}+4$ \\
\hline & & & & & Varic & & & & & \\
\hline 0 & $2.702 E+1$ & $8.206 \mathrm{E}+0$ & 9.373E-1 & $1.600 \mathrm{E}+1$ & 7 & & 3.2 & & & $9.322 \mathrm{E}$ \\
\hline & & & & & Varic & & & & & \\
\hline 0 & $1.525 \mathrm{E}+1$ & $8.119 \mathrm{E}+0$ & 8.524E-1 & $8.865 E+0$ & $2.770 \mathrm{E}+1 \quad 2.333 \mathrm{E}+1$ & 3. & $1.463 \mathrm{E}_{+}$ & $2.102 \mathrm{E}$ & $8 \mathrm{E}+$ & $3.019 \mathrm{E}+$ \\
\hline 10 & $1.513 E+1$ & $7.412 \mathrm{E}+0$ & $8.528 \mathrm{E}-1$ & $8.780 \mathrm{E}+0$ & $2.800 \mathrm{E}+1 \quad 2.275 \mathrm{E}+1$ & $3.158 \mathrm{E}+1$ & $1.434 \mathrm{E}+1$ & $2.058 \mathrm{E}+4$ & $6.884 \mathrm{E}+2$ & $6.093 E+0$ \\
\hline 20 & $1.525 \mathrm{E}+1$ & $7.132 \mathrm{E}+0$ & 8.541E-1 & $8.867 E+0$ & $2.886 \mathrm{E}+1 \quad 2.253 \mathrm{E}+1$ & $3.202 \mathrm{E}+1$ & $1.406 \mathrm{E}+1$ & $2.122 E+4$ & $7.086 \mathrm{E}+2$ & $4.690 \mathrm{E}+0$ \\
\hline 30 & $1.541 \mathrm{E}+1$ & $6.937 \mathrm{E}+0$ & $8.557 \mathrm{E}-1$ & $8.979 E+0$ & $2.982 \mathrm{E}+1 \quad 2.253 \mathrm{E}+1$ & $3.255 \mathrm{E}+1$ & $1.375 \mathrm{E}+1$ & $2.202 \mathrm{E}+4$ & $7.336 \mathrm{E}+2$ & $4.105 E+0$ \\
\hline 40 & $1.560 \mathrm{E}+1$ & $6.791 \mathrm{E}+0$ & 8.574E-1 & $9.108 \mathrm{E}+0$ & $3.085 \mathrm{E}+1 \quad 2.268 \mathrm{E}+1$ & $3.314 \mathrm{E}+1$ & $1.346 \mathrm{E}+1$ & $2.292 \mathrm{E}+4$ & $7.617 \mathrm{E}+2$ & 3.75 \\
\hline 50 & $1.581 \mathrm{E}+1$ & $6.683 \mathrm{E}+0$ & 8.595E-1 & $9.249 \mathrm{E}+0$ & $3.193 \mathrm{E}+1 \quad 2.298 \mathrm{E}+1$ & $3.380 \mathrm{E}+1$ & $1.318 \mathrm{E}+1$ & $2.389 \mathrm{E}+4$ & $7.921 E+2$ & $3.511 E+0$ \\
\hline 60 & $1.602 E+1$ & $6.596 \mathrm{E}+0$ & $8.615 \mathrm{E}-1$ & $9.395 E+0$ & $3.302 \mathrm{E}+1 \quad 2.339 \mathrm{E}+1$ & $3.449 \mathrm{E}+1$ & $1.290 \mathrm{E}+1$ & $2.490 \mathrm{E}+4$ & $8.238 \mathrm{E}+2$ & $3.321 \mathrm{E}+0$ \\
\hline
\end{tabular}


Table 4.27. Fuel-averaged microscopic production cross section for uranium and plutonium isotopes vs burnup, Variant $1-4$ and 7-10, State 1

\begin{tabular}{|c|c|c|c|c|c|c|c|c|}
\hline \multirow{2}{*}{$\begin{array}{c}\text { Burnup } \\
\text { (MWd/kg) }\end{array}$} & \multicolumn{8}{|c|}{ Microscopic production cross section (b) } \\
\hline & ${ }^{235} \mathrm{U}$ & ${ }^{236} \mathrm{U}$ & ${ }^{238} \mathrm{U}$ & ${ }^{238} \mathrm{Pu}$ & ${ }^{239} \mathrm{Pu}$ & ${ }^{240} \mathrm{Pu}$ & ${ }^{241} \mathrm{Pu}$ & ${ }^{242} \mathrm{Pu}$ \\
\hline & & & & Variant 1 & & & & \\
\hline 0 & $8.454 E+1$ & $7.972 \mathrm{E}-1$ & 2.813E-1 & $6.507 \mathrm{E}+0$ & $2.710 \mathrm{E}+2$ & $1.884 \mathrm{E}+0$ & $2.796 \mathrm{E}+2$ & $1.385 E+0$ \\
\hline 10 & $7.669 \mathrm{E}+1$ & $7.810 \mathrm{E}-1$ & $2.894 \mathrm{E}-1$ & $6.394 \mathrm{E}+0$ & $2.313 E+2$ & $1.894 \mathrm{E}+0$ & $2.474 \mathrm{E}+2$ & $1.415 E+0$ \\
\hline 20 & $7.392 \mathrm{E}+1$ & $7.720 \mathrm{E}-1$ & $2.937 \mathrm{E}-1$ & $6.371 \mathrm{E}+0$ & $2.150 \mathrm{E}+2$ & $1.891 \mathrm{E}+0$ & $2.344 \mathrm{E}+2$ & $1.431 \mathrm{E}+0$ \\
\hline 30 & $7.325 E+1$ & $7.655 \mathrm{E}-1$ & $2.959 \mathrm{E}-1$ & $6.382 E+0$ & $2.083 E+2$ & $1.888 \mathrm{E}+0$ & $2.298 \mathrm{E}+2$ & $1.439 E+0$ \\
\hline 40 & $7.346 \mathrm{E}+1$ & $7.618 \mathrm{E}-1$ & $2.974 \mathrm{E}-1$ & $6.406 \mathrm{E}+0$ & $2.058 \mathrm{E}+2$ & $1.887 \mathrm{E}+0$ & $2.287 E+2$ & $1.443 E+0$ \\
\hline 50 & $7.395 \mathrm{E}+1$ & $7.600 \mathrm{E}-1$ & $2.989 E-1$ & $6.434 \mathrm{E}+0$ & $2.051 E+2$ & $1.887 \mathrm{E}+0$ & $2.291 E+2$ & $1.447 \mathrm{E}+0$ \\
\hline 60 & $7.440 \mathrm{E}+1$ & $7.597 \mathrm{E}-1$ & $3.002 \mathrm{E}-1$ & $6.457 \mathrm{E}+0$ & $2.049 E+2$ & $1.889 \mathrm{E}+0$ & $2.297 \mathrm{E}+2$ & $1.451 \mathrm{E}+0$ \\
\hline & & & & Variant 2 & & & & \\
\hline 0 & $4.748 \mathrm{E}+01$ & 8.673E-01 & $3.134 \mathrm{E}-01$ & $5.824 \mathrm{E}+00$ & $1.094 E+02$ & $1.973 E+00$ & $1.349 E+02$ & $1.512 E+00$ \\
\hline 10 & 4.877E+01 & $8.571 \mathrm{E}-01$ & 3.129E-01 & $5.873 E+00$ & $1.168 \mathrm{E}+02$ & $1.953 E+00$ & $1.416 E+02$ & $1.510 \mathrm{E}+00$ \\
\hline 20 & $5.156 \mathrm{E}+01$ & $8.482 \mathrm{E}-01$ & $3.114 \mathrm{E}-01$ & $5.946 \mathrm{E}+00$ & $1.276 \mathrm{E}+02$ & $1.941 E+00$ & $1.515 E+02$ & $1.503 \mathrm{E}+00$ \\
\hline 30 & $5.454 \mathrm{E}+01$ & 8.397E-01 & $3.098 \mathrm{E}-01$ & $6.018 \mathrm{E}+00$ & $1.387 \mathrm{E}+02$ & $1.930 \mathrm{E}+00$ & $1.618 E+02$ & $1.496 \mathrm{E}+00$ \\
\hline 40 & $5.745 E+01$ & 8.334E-01 & $3.082 E-01$ & $6.087 E+00$ & $1.491 E+02$ & $1.922 \mathrm{E}+00$ & $1.718 E+02$ & $1.490 \mathrm{E}+00$ \\
\hline 50 & $6.010 \mathrm{E}+01$ & 8.285E-01 & 3.074E-01 & $6.150 \mathrm{E}+00$ & $1.582 \mathrm{E}+02$ & $1.917 \mathrm{E}+00$ & $1.807 \mathrm{E}+02$ & $1.485 E+00$ \\
\hline 60 & $6.241 E+01$ & $8.250 \mathrm{E}-01$ & 3.071E-01 & $6.206 \mathrm{E}+00$ & $1.659 \mathrm{E}+02$ & $1.914 \mathrm{E}+00$ & $1.885 E+02$ & $1.483 E+00$ \\
\hline & & & & Variant 3 & & & & \\
\hline 0 & $7.723 \mathrm{E}+1$ & 7.713E-1 & $2.929 \mathrm{E}-1$ & $6.458 \mathrm{E}+0$ & $2.176 \mathrm{E}+2$ & $1.872 \mathrm{E}+0$ & $2.409 \mathrm{E}+2$ & $1.425 \mathrm{E}+0$ \\
\hline & & & & Variant 4 & & & & \\
\hline 0 & $7.320 \mathrm{E}+1$ & $7.699 \mathrm{E}-1$ & $2.961 \mathrm{E}-1$ & $6.382 \mathrm{E}+0$ & $2.061 \mathrm{E}+2$ & $1.889 \mathrm{E}+0$ & $2.280 \mathrm{E}+2$ & $1.441 \mathrm{E}+0$ \\
\hline & & & & Variant 7 & & & & \\
\hline 0 & $5.137 \mathrm{E}+1$ & 8.593E-1 & 3.099E-1 & $5.867 \mathrm{E}+0$ & $1.191 \mathrm{E}+2$ & $2.029 \mathrm{E}+0$ & $1.447 \mathrm{E}+2$ & $1.495 \mathrm{E}+0$ \\
\hline & & & & Variant 8 & & & & \\
\hline 0 & $.021 E+1$ & $7.944 \mathrm{E}-1$ & $2.775 \mathrm{E}-1$ & $6.679 E+0$ & $2.842 E+2$ & $1.802 \mathrm{E}+0$ & $2.980 \mathrm{E}+2$ & 1.37 \\
\hline & & & & Variant 9 & & & & \\
\hline 0 & $5.114 \mathrm{E}+1$ & $8.199 \mathrm{E}-1$ & $2.985 \mathrm{E}-1$ & $5.790 \mathrm{E}+0$ & $1.448 \mathrm{E}+2$ & $1.981 \mathrm{E}+0$ & $1.460 \mathrm{E}+2$ & $1.458 \mathrm{E}+0$ \\
\hline & & & & Variant 10 & & & & \\
\hline 0 & $2.698 \mathrm{E}+1$ & 8.647E-1 & 3.245E-1 & $5.450 \mathrm{E}+0$ & $5.130 \mathrm{E}+1$ & $2.013 E+0$ & $7.145 \mathrm{E}+1$ & $1.577 \mathrm{E}+0$ \\
\hline 10 & $2.675 E+1$ & $8.460 \mathrm{E}-1$ & $3.248 \mathrm{E}-1$ & $5.451 E+0$ & $5.173 E+1$ & $2.015 \mathrm{E}+0$ & $7.128 \mathrm{E}+1$ & $1.579 \mathrm{E}+0$ \\
\hline 20 & $2.701 \mathrm{E}+1$ & 8.387E-1 & $3.249 \mathrm{E}-1$ & $5.461 \mathrm{E}+0$ & $5.322 \mathrm{E}+1$ & $2.016 \mathrm{E}+0$ & $7.225 E+1$ & $1.579 \mathrm{E}+0$ \\
\hline 30 & $2.735 \mathrm{E}+1$ & 8.335E-1 & $3.250 \mathrm{E}-1$ & $5.472 \mathrm{E}+0$ & $5.493 \mathrm{E}+1$ & $2.016 \mathrm{E}+0$ & $7.339 \mathrm{E}+1$ & $1.579 \mathrm{E}+0$ \\
\hline 40 & $2.774 \mathrm{E}+1$ & 8.296E-1 & $3.251 \mathrm{E}-1$ & $5.483 E+0$ & $5.676 \mathrm{E}+1$ & $2.017 \mathrm{E}+0$ & $7.468 \mathrm{E}+1$ & $1.579 \mathrm{E}+0$ \\
\hline 50 & $2.818 \mathrm{E}+1$ & 8.264E-1 & $3.250 \mathrm{E}-1$ & $5.494 \mathrm{E}+0$ & $5.869 \mathrm{E}+1$ & $2.016 \mathrm{E}+0$ & $7.611 \mathrm{E}+1$ & $1.579 \mathrm{E}+0$ \\
\hline 60 & $2.864 \mathrm{E}+1$ & 8.242E-1 & $3.251 \mathrm{E}-1$ & $5.505 \mathrm{E}+0$ & $6.066 \mathrm{E}+1$ & $2.017 \mathrm{E}+0$ & $7.761 E+1$ & $1.579 E+0$ \\
\hline
\end{tabular}


Table 4.28. $k_{\text {efr }}$ and $k_{0}$ values for Variants 15-18, States 7-12

\begin{tabular}{|c|c|c|c|c|c|c|c|c|c|c|c|c|}
\hline \multirow{3}{*}{ Variant } & \multicolumn{12}{|c|}{ State } \\
\hline & \multicolumn{2}{|c|}{ S7 } & \multicolumn{2}{|c|}{ S8 } & \multicolumn{2}{|c|}{ S9 } & \multicolumn{2}{|c|}{ S10 } & \multicolumn{2}{|c|}{ S11 } & \multicolumn{2}{|c|}{$\mathrm{S} 12$} \\
\hline & $k_{\text {eff }}$ & $\mathrm{k}_{0}$ & $\mathrm{k}_{\text {eff }}$ & $\mathrm{k}_{0}$ & $\mathrm{k}_{\text {eff }}$ & $\mathrm{k}_{0}$ & $\mathrm{k}_{\text {eff }}$ & $\mathrm{k}_{0}$ & $\mathrm{k}_{\text {eff }}$ & $\mathrm{k}_{0}$ & $\mathrm{k}_{\text {eff }}$ & $\mathrm{k}_{0}$ \\
\hline V15 & 1.3846 & 1.3727 & 1.3561 & 1.3445 & 1.0748 & 1.0737 & 1.2669 & 1.2607 & 1.0000 & 1.3727 & 1.0000 & 1.4578 \\
\hline V16 & 1.1107 & 1.1084 & 1.0818 & 1.0800 & 0.8081 & 0.8048 & 1.0250 & 1.0246 & 1.0000 & 1.1084 & 1.0000 & 1.2026 \\
\hline V17 & 1.2682 & 1.2644 & 1.2341 & 1.2306 & 0.9732 & 0.9727 & 1.2085 & 1.2061 & 1.0000 & 1.2644 & 1.0000 & 1.3818 \\
\hline V18 & 1.0809 & 1.0798 & 1.0498 & 1.0491 & 0.7911 & 0.7853 & 1.0164 & 1.0162 & 1.0000 & 1.0798 & 1.0000 & 1.1771 \\
\hline V19 & 1.3743 & 1.3743 & 1.3457 & 1.3457 & 1.0684 & 1.0684 & 1.2619 & 1.2619 & 1.0000 & 1.3646 & 1.0000 & 1.4521 \\
\hline $\mathrm{V} 20$ & 1.1759 & 1.1759 & 1.1471 & 1.1471 & 0.8706 & 0.8706 & 1.0888 & 1.0888 & 1.0000 & 1.1726 & 1.0000 & 1.2677 \\
\hline
\end{tabular}

Table 4.29. Values of critical buckling for Variants 15-18, States 11 and 12

\begin{tabular}{ccccccc}
\hline \multirow{2}{*}{ State } & \multicolumn{7}{c}{ Critical buckling $\left(\mathrm{cm}^{-2}\right)$} \\
\cline { 2 - 7 } & Variant 15 & Variant 16 & Variant 17 & Variant 18 & Variant 19 & Variant 20 \\
\hline S11 & $5.607 \mathrm{E}-3$ & $1.694 \mathrm{E}-3$ & $4.131 \mathrm{E}-3$ & $1.270 \mathrm{E}-3$ & $5.568 \mathrm{E}-3$ & $2.706 \mathrm{E}-3$ \\
S12 & $1.049 \mathrm{E}-2$ & $4.772 \mathrm{E}-3$ & $8.949 \mathrm{E}-3$ & $4.234 \mathrm{E}-3$ & $1.054 \mathrm{E}-2$ & $6.362 \mathrm{E}-3$ \\
\hline
\end{tabular}


Table 4.30. Fuel-averaged microscopic production cross sections $\left(v \sigma_{f}\right)$ for uranium and plutonium isotopes for Variants 15-18

\begin{tabular}{|c|c|c|c|c|c|c|c|c|}
\hline \multirow{2}{*}{ State } & \multicolumn{8}{|c|}{ Fuel-averaged microscopic production cross section (b) } \\
\hline & ${ }^{235} \mathrm{U}$ & ${ }^{236} \mathrm{U}$ & ${ }^{238} \mathrm{U}$ & ${ }^{238} \mathrm{Pu}$ & ${ }^{239} \mathrm{Pu}$ & ${ }^{240} \mathrm{Pu}$ & ${ }^{241} \mathrm{Pu}$ & ${ }^{242} \mathrm{Pu}$ \\
\hline \multicolumn{9}{|c|}{ Variant 15} \\
\hline S7 & $8.749 \mathrm{E}+1$ & 7.964E-1 & $2.849 \mathrm{E}-1$ & $6.605 \mathrm{E}+0$ & $2.761 E+2$ & $1.901 \mathrm{E}+0$ & $2.877 \mathrm{E}+2$ & $1.403 E+0$ \\
\hline S8 & $8.660 \mathrm{E}+1$ & $7.938 \mathrm{E}-1$ & 2.854E-1 & $6.570 \mathrm{E}+0$ & 2.797E+2 & $1.903 E+0$ & $2.877 E+2$ & $1.406 \mathrm{E}+0$ \\
\hline S9 & $3.028 \mathrm{E}+1$ & 6.377E-1 & $2.204 \mathrm{E}-1$ & $5.124 \mathrm{E}+0$ & $9.225 \mathrm{E}+1$ & $1.687 \mathrm{E}+0$ & $9.275 \mathrm{E}+1$ & $1.231 \mathrm{E}+0$ \\
\hline $\mathbf{S 1 0}$ & $8.279 \mathrm{E}+1$ & 7.997E-1 & 2.841E-1 & $6.481 \mathrm{E}+0$ & $2.640 \mathrm{E}+2$ & $1.899 \mathrm{E}+0$ & $2.730 \mathrm{E}+2$ & $1.399 \mathrm{E}+0$ \\
\hline $\mathrm{S} 11$ & 8.751E+1 & 7.962E-1 & $2.848 \mathrm{E}-1$ & $6.605 \mathrm{E}+0$ & $2.761 E+2$ & $1.901 \mathrm{E}+0$ & $2.878 \mathrm{E}+2$ & $1.403 \mathrm{E}+0$ \\
\hline $\mathrm{S} 12$ & $8 E+2$ & 8.270E-1 & $2.976 \mathrm{E}-1$ & $7.724 \mathrm{E}+0$ & $2.915 E+2$ & $1.940 \mathrm{E}+0$ & $3.300 \mathrm{E}+2$ & $1.435 E+0$ \\
\hline \multicolumn{9}{|c|}{ Variant 16} \\
\hline S7 & $8.068 \mathrm{E}+1$ & $7.218 \mathrm{E}-1$ & 2.904E-1 & $6.557 \mathrm{E}+0$ & $2.250 E+2$ & $1.852 \mathrm{E}+0$ & $2.508 E+2$ & $1.412 \mathrm{E}+0$ \\
\hline S8 & $7.855 E+1$ & $\cdot 7.360 \mathrm{E}-1$ & 2.917E-1 & $6.498 \mathrm{E}+0$ & $2.249 E+2$ & $1.863 E+0$ & $2.469 E+2$ & $1.418 \mathrm{E}+0$ \\
\hline S9 & $2.739 E+1$ & $5.592 \mathrm{E}-1$ & $2.140 \mathrm{E}-1$ & $5.133 E+0$ & $6.390 \mathrm{E}+1$ & $1.590 \mathrm{E}+0$ & $7.510 \mathrm{E}+1$ & $1.187 \mathrm{E}+0$ \\
\hline S10 & $7.592 \mathrm{E}+1$ & $7.244 \mathrm{E}-1$ & $2.907 \mathrm{E}-1$ & $6.433 E+0$ & $2.136 E+2$ & $1.853 E+0$ & $2.367 \mathrm{E}+2$ & $1.411 \mathrm{E}+0$ \\
\hline S11 & $8.066 \mathrm{E}+1$ & $7.221 \mathrm{E}-1$ & $2.906 \mathrm{E}-1$ & $6.557 E+0$ & $2.249 E+2$ & $1.853 \mathrm{E}+0$ & $2.508 E+2$ & $1.412 E+0$ \\
\hline S12 & $1.231 \mathrm{E}+2$ & $7.226 \mathrm{E}-1$ & $3.010 \mathrm{E}-1$ & $7.955 \mathrm{E}+0$ & $2.733 E+2$ & $1.870 \mathrm{E}+0$ & $3.273 E+2$ & $1.430 \mathrm{E}+0$ \\
\hline \multicolumn{9}{|c|}{ Variant 17} \\
\hline S7 & $4.874 \mathrm{E}+1$ & 8.672E-1 & $3.148 \mathrm{E}-1$ & $5.864 \mathrm{E}+0$ & $1.118 \mathrm{E}+2$ & $1.977 \mathrm{E}+0$ & $1.382 E+2$ & $1.519 \mathrm{E}+0$ \\
\hline S8 & $4.716 \mathrm{E}+1$ & $8.658 \mathrm{E}-1$ & $3.159 \mathrm{E}-1$ & $5.829 E+0$ & $1.103 E+2$ & $1.988 \mathrm{E}+0$ & $1.345 E+2$ & $1.524 E+0$ \\
\hline 59 & $2.165 E+1$ & $6.627 \mathrm{E}-1$ & $2.288 \mathrm{E}-1$ & $5.025 \mathrm{E}+0$ & $3.530 E+1$ & $1.661 \mathrm{E}+0$ & $5.247 \mathrm{E}+1$ & $1.249 \mathrm{E}+0$ \\
\hline S10 & $4.713 E+1$ & 8.679E-1 & 3.141E-1 & $5.821 \mathrm{E}+0$ & $1.080 \mathrm{E}+2$ & $1.973 E+0$ & $1.336 \mathrm{E}+2$ & $1.515 E+0$ \\
\hline S11 & $74 \mathrm{E}+1$ & 8.673E-1 & 3.149E-1 & $5.864 \mathrm{E}+0$ & $1.118 \mathrm{E}+2$ & $1.978 \mathrm{E}+0$ & $1.382 E+2$ & $1.519 \mathrm{E}+0$ \\
\hline S12 & $7.534 \mathrm{E}+1$ & 8.929E-1 & $3.266 \mathrm{E}-1$ & $6.691 \mathrm{E}+0$ & $1.540 \mathrm{E}+2$ & $2.003 E+0$ & $1.962 \mathrm{E}+2$ & $1.544 \mathrm{E}+0$ \\
\hline \multicolumn{9}{|c|}{ Variant 18} \\
\hline S7 & $6.253 \mathrm{E}+1$ & $8.095 \mathrm{E}-1$ & $3.027 \mathrm{E}-1$ & $6.188 \mathrm{E}+0$ & $1.569 \mathrm{E}+2$ & $1.883 \mathrm{E}+0$ & $1.844 \mathrm{E}+2$ & $1.460 \mathrm{E}+0$ \\
\hline S8 & $6.027 \mathrm{E}+1$ & 8.138E-1 & 3.043E-1 & $6.135 E+0$ & $1.550 \mathrm{E}+2$ & $1.893 \mathrm{E}+0$ & $1.796 \mathrm{E}+2$ & $1.466 \mathrm{E}+0$ \\
\hline$\$ 9$ & $2.444 \mathrm{E}+1$ & $6.195 \mathrm{E}-1$ & $2.159 \mathrm{E}-1$ & $5.092 \mathrm{E}+0$ & $4.566 \mathrm{E}+1$ & $1.577 \mathrm{E}+0$ & $6.151 E+1$ & $1.187 \mathrm{E}+0$ \\
\hline S10 & $5.956 \mathrm{E}+1$ & $8.114 \mathrm{E}-1$ & $3.026 \mathrm{E}-1$ & $6.110 \mathrm{E}+0$ & $1.501 \mathrm{E}+2$ & $1.881 E+0$ & $1.759 \mathrm{E}+2$ & $1.458 \mathrm{E}+0$ \\
\hline S11 & $6.252 \mathrm{E}+1$ & 8.095E-1 & 3.027E-1 & $6.188 \mathrm{E}+0$ & $1.569 \mathrm{E}+2$ & $1.883 \mathrm{E}+0$ & $1.844 \mathrm{E}+2$ & $1.460 \mathrm{E}+0$ \\
\hline $\mathrm{S} 12$ & $1.010 \mathrm{E}+2$ & $8.202 \mathrm{E}-1$ & $3.118 \mathrm{E}-1$ & $7.397 \mathrm{E}+0$ & $2.129 \mathrm{E}+2$ & $1.894 \mathrm{E}+0$ & $2.633 E+2$ & $1.472 E+0$ \\
\hline
\end{tabular}


Table 4.31. Cell relative production rates for uranium and plutonium isotopes for Variants 15-18

\begin{tabular}{|c|c|c|c|c|c|c|c|c|}
\hline \multirow[b]{2}{*}{ State } & \multicolumn{8}{|c|}{ Cell relative production rate } \\
\hline & ${ }^{235} \mathbf{U}$ & ${ }^{236} \mathrm{U}$ & ${ }^{238} \mathrm{U}$ & ${ }^{238} \mathrm{Pu}$ & ${ }^{239} \mathbf{P u}$ & ${ }^{240} \mathbf{P u}$ & ${ }^{241} \mathbf{P u}$ & ${ }^{242} \mathrm{Pu}$ \\
\hline \multicolumn{9}{|c|}{ Variant 15} \\
\hline S7 & 9.347E-1 & 0.0 & $6.530 \mathrm{E}-2$ & 0.0 & 0.0 & 0.0 & 0.0 & 0.0 \\
\hline S8 & $9.340 \mathrm{E}-1$ & 0.0 & $6.604 \mathrm{E}-2$ & 0.0 & 0.0 & 0.0 & 0.0 & 0.0 \\
\hline S9 & $8.649 \mathrm{E}-1$ & 0.0 & $1.351 \mathrm{E}-1$ & 0.0 & 0.0 & 0.0 & 0.0 & 0.0 \\
\hline $\mathbf{S 1 0}$ & $9.314 \mathrm{E}-1$ & 0.0 & $6.857 \mathrm{E}-2$ & 0.0 & 0.0 & 0.0 & 0.0 & 0.0 \\
\hline S11 & $9.347 \mathrm{E}-1$ & 0.0 & $6.527 \mathrm{E}-2$ & 0.0 & 0.0 & 0.0 & 0.0 & 0.0 \\
\hline S12 & $9.490 \mathrm{E}-1$ & 0.0 & $5.099 \mathrm{E}-2$ & 0.0 & 0.0 & 0.0 & 0.0 & 0.0 \\
\hline \multicolumn{9}{|c|}{ Variant 16} \\
\hline S7 & 4.299E-1 & $8.778 \mathrm{E}-4$ & $7.493 \mathrm{E}-2$ & $6.209 \mathrm{E}-4$ & 4.153E-1 & $9.449 \mathrm{E}-4$ & $7.665 \mathrm{E}-2$ & $9.458 \mathrm{E}-5$ \\
\hline S8 & $4.238 \mathrm{E}-1$ & $9.063 \mathrm{E}-4$ & $7.620 \mathrm{E}-2$ & $6.230 \mathrm{E}-4$ & $4.203 \mathrm{E}-1$ & $9.622 \mathrm{E}-4$ & $7.639 \mathrm{E}-2$ & $9.614 \mathrm{E}-5$ \\
\hline S9 & $4.234 \mathrm{E}-1$ & $1.973 E-3$ & $1.602 \mathrm{E}-1$ & $1.410 \mathrm{E}-3$ & $3.422 \mathrm{E}-1$ & 2.353E-3 & 6.657E-2 & $2.308 \mathrm{E}-4$ \\
\hline S10 & $4.261 \mathrm{E}-1$ & $9.279 \mathrm{E}-4$ & $7.901 \mathrm{E}-2$ & $6.417 \mathrm{E}-4$ & 4.153E-1 & $9.955 \mathrm{E}-4$ & 7.617E-2 & $9.958 \mathrm{E}-5$ \\
\hline S11 & 4.299E-1 & $8.782 \mathrm{E}-4$ & 7.499E-2 & $6.210 \mathrm{E}-4$ & 4.153E-1 & $9.453 E-4$ & $7.665 \mathrm{E}-2$ & 9.462E-5 \\
\hline $\mathrm{S} 12$ & 4.889E-1 & $6.551 E-4$ & 5.791E-2 & $5.615 \mathrm{E}-4$ & $3.761 \mathrm{E}-1$ & $7.113 \mathrm{E}-4$ & $7.455 \mathrm{E}-2$ & 7.142E-5 \\
\hline \multicolumn{9}{|c|}{ Variant 17} \\
\hline S7 & $2.268 \mathrm{E}-2$ & 0.0 & $7.217 \mathrm{E}-2$ & 0.0 & 8.924E-1 & $1.014 \mathrm{E}-3$ & $1.177 \mathrm{E}-2$ & 0.0 \\
\hline S8 & 2.222E-2 & 0.0 & $7.334 \mathrm{E}-2$ & 0.0 & $8.918 \mathrm{E}-1$ & $1.032 \mathrm{E}-3$ & $1.159 \mathrm{E}-2$ & 0.0 \\
\hline S9 & 2.881E-2 & 0.0 & $1.500 \mathrm{E}-1$ & 0.0 & $8.060 \mathrm{E}-1$ & $2.436 \mathrm{E}-3$ & $1.278 \mathrm{E}-2$ & 0.0 \\
\hline $\mathrm{S} 10$ & $2.264 \mathrm{E}-2$ & 0.0 & $7.431 \mathrm{E}-2$ & 0.0 & $8.903 \mathrm{E}-1$ & $1.045 \mathrm{E}-3$ & $1.174 \mathrm{E}-2$ & 0.0 \\
\hline S11 & $2.268 \mathrm{E}-2$ & 0.0 & $7.219 \mathrm{E}-2$ & 0.0 & 8.924E-1 & $1.014 \mathrm{E}-3$ & $1.177 \mathrm{E}-2$ & 0.0 \\
\hline $\mathrm{S} 12$ & $2.583 \mathrm{E}-2$ & 0.0 & $5.517 \mathrm{E}-2$ & 0.0 & $9.059 \mathrm{E}-1$ & $7.568 \mathrm{E}-4$ & $1.231 \mathrm{E}-2$ & 0.0 \\
\hline \multicolumn{9}{|c|}{ Variant 18} \\
\hline S7 & $1.742 \mathrm{E}-2$ & 4.007E-5 & 7.797E-2 & $9.643 \mathrm{E}-5$ & 7.041E-1 & $3.842 \mathrm{E}-3$ & $1.956 \mathrm{E}-1$ & $3.520 \mathrm{E}-4$ \\
\hline S8 & $1.703 \mathrm{E}-2$ & $4.086 \mathrm{E}-5$ & 7.949E-2 & $9.697 \mathrm{E}-5$ & $7.054 \mathrm{E}-1$ & $3.919 \mathrm{E}-3$ & 1.931E-1 & $3.586 E-4$ \\
\hline S9 & $2.024 \mathrm{E}-2$ & $9.116 \mathrm{E}-5$ & 1.653E-1 & $2.358 \mathrm{E}-4$ & $6.089 E-1$ & $9.562 \mathrm{E}-3$ & $1.939 \mathrm{E}-1$ & $8.509 \mathrm{E}-4$ \\
\hline S10 & $1.730 \mathrm{E}-2$ & $4.188 \mathrm{E}-5$ & $8.124 \mathrm{E}-2$ & $9.925 \mathrm{E}-5$ & $7.020 \mathrm{E}-1$ & $4.001 E-3$ & $1.944 \mathrm{E}-1$ & $3.664 \mathrm{E}-4$ \\
\hline S11 & $1.742 \mathrm{E}-2$ & $4.008 \mathrm{E}-5$ & 7.797E-2 & $9.643 \mathrm{E}-5$ & $7.041 \mathrm{E}-1$ & $3.842 \mathrm{E}-3$ & $1.956 \mathrm{E}-1$ & $3.520 \mathrm{E}-4$ \\
\hline $\mathrm{S} 12$ & $2.088 \mathrm{E}-2$ & $3.013 \mathrm{E}-5$ & $5.958 \mathrm{E}-2$ & $8.551 \mathrm{E}-5$ & $7.086 \mathrm{E}-1$ & $2.867 \mathrm{E}-3$ & $2.071 \mathrm{E}-1$ & $2.633 \mathrm{E}-4$ \\
\hline
\end{tabular}


Table 4.32. Fuel-averaged microscopic absorption cross sections $\left(\sigma_{\mathrm{a}}\right)$ for uranium and plutonium isotopes for Variants $15-18$

\begin{tabular}{|c|c|c|c|c|c|c|c|c|c|c|c|}
\hline \multirow{2}{*}{ State } & \multicolumn{11}{|c|}{ Fuel-averaged microscopic absorption cross section (b) } \\
\hline & ${ }^{235} \mathrm{U}$ & ${ }^{236} \mathrm{U}$ & ${ }^{238} \mathrm{U}$ & ${ }^{238} \mathrm{Pu}$ & ${ }^{239} \mathrm{Pu}$ & ${ }^{240} \mathrm{Pu}$ & ${ }^{241} \mathrm{Pu}$ & ${ }^{242} \mathrm{Pu}$ & ${ }^{135} \mathrm{Xe}$ & ${ }^{149} \mathrm{Sm}$ & All FP \\
\hline \multicolumn{12}{|c|}{ Variant 15} \\
\hline S7 & $4.426 \mathrm{E}+1$ & $8.359 \mathrm{E}+0$ & $9.585 \mathrm{E}-1$ & $2.847 \mathrm{E}+1$ & $1.516 \mathrm{E}+2$ & $2.172 E+2$ & $1.308 E+2$ & $3.238 \mathrm{E}+1$ & $1.523 E+5$ & $4.808 \mathrm{E}+3$ & $1.550 \mathrm{E}+3$ \\
\hline S8 & $4.384 \mathrm{E}+1$ & $8.231 \mathrm{E}+0$ & $1.015 \mathrm{E}+0$ & $2.805 \mathrm{E}+1$ & $1.539 \mathrm{E}+2$ & $2.161 E+2$ & $1.309 \mathrm{E}+2$ & $3.228 \mathrm{E}+1$ & $1.495 \mathrm{E}+5$ & $4.714 \mathrm{E}+3$ & $1.522 \mathrm{E}+3$ \\
\hline S9 & $1.642 \mathrm{E}+1$ & $6.253 \mathrm{E}+0$ & $6.987 \mathrm{E}-1$ & $9.463 \mathrm{E}+0$ & $5.156 \mathrm{E}+1$ & $1.339 \mathrm{E}+2$ & 4.196E+1 & $2.395 \mathrm{E}+1$ & $2.581 E+4$ & $8.872 \mathrm{E}+2$ & $2.815 E+2$ \\
\hline S10 & $4.205 \mathrm{E}+1$ & $8.520 \mathrm{E}+0$ & $9.619 \mathrm{E}-1$ & $2.675 E+1$ & $1.452 \mathrm{E}+2$ & $2.196 \mathrm{E}+2$ & $1.242 \mathrm{E}+2$ & $3.299 \mathrm{E}+1$ & $1.399 \mathrm{E}+5$ & $4.435 E+3$ & $1.427 \mathrm{E}+3$ \\
\hline S11 & $4.427 \mathrm{E}+1$ & $8.359 E+0$ & $9.585 \mathrm{E}-1$ & $2.847 \mathrm{E}+1$ & $1.516 \mathrm{E}+2$ & $2.172 E+2$ & $1.309 E+2$ & $3.238 \mathrm{E}+1$ & $1.524 \mathrm{E}+5$ & $4.809 \mathrm{E}+3$ & $1.550 \mathrm{E}+3$ \\
\hline S12 & $5.929 \mathrm{E}+1$ & $8.804 \mathrm{E}+0$ & $9.797 \mathrm{E}-1$ & $4.191 \mathrm{E}+1$ & $1.547 \mathrm{E}+2$ & $2.312 \mathrm{E}+2$ & $1.501 \mathrm{E}+2$ & 3.380E+1 & $2.250 \mathrm{E}+5$ & $6.030 \mathrm{E}+3$ & $2.276 \mathrm{E}+3$ \\
\hline \multicolumn{12}{|c|}{ Variant 16} \\
\hline S7 & $4.113 \mathrm{E}+1$ & $5.437 \mathrm{E}+0$ & $9.960 \mathrm{E}-1$ & $2.682 E+1$ & $1.223 E+2$ & $1.091 E+2$ & $1.135 E+2$ & $2.846 \mathrm{E}+1$ & $1.402 E+5$ & $4.373 E+3$ & $.321 \mathrm{E}+1$ \\
\hline S8 & $4.010 \mathrm{E}+1$ & $5.797 \mathrm{E}+0$ & $1.057 \mathrm{E}+0$ & $2.600 \mathrm{E}+1$ & $1.226 \mathrm{E}+2$ & $1.147 \mathrm{E}+2$ & $1.119 \mathrm{E}+2$ & $2.971 E+1$ & $1.347 \mathrm{E}+5$ & $4.201 E+3$ & $1.308 \mathrm{E}+1$ \\
\hline S9 & $1.530 \mathrm{E}+1$ & $4.005 E+0$ & $7.408 \mathrm{E}-1$ & $8.872 \mathrm{E}+0$ & $3.535 \mathrm{E}+1$ & $4.716 \mathrm{E}+1$ & $3.359 E+1$ & $2.079 \mathrm{E}+1$ & $2.025 E+4$ & $6.929 \mathrm{E}+2$ & $7.689 \mathrm{E}+0$ \\
\hline S10 & $3.889 \mathrm{E}+1$ & $5.510 \mathrm{E}+0$ & $9.983 \mathrm{E}-1$ & $2.507 \mathrm{E}+1$ & $1.164 E+2$ & $1.091 E+2$ & $1.071 E+2$ & $2.884 E+1$ & $1.277 \mathrm{E}+5$ & $4.003 E+3$ & $1.300 \mathrm{E}+1$ \\
\hline S11 & $4.112 \mathrm{E}+1$ & $5.437 \mathrm{E}+0$ & $9.959 \mathrm{E}-1$ & $2.682 \mathrm{E}+1$ & $1.223 E+2$ & $1.091 \mathrm{E}+2$ & $1.135 E+2$ & $2.845 E+1$ & $1.401 \mathrm{E}+5$ & $4.372 E+3$ & $1.321 \mathrm{E}+1$ \\
\hline S12 & $6.145 E+1$ & $5.088 \mathrm{E}+0$ & $1.022 \mathrm{E}+0$ & $4.424 \mathrm{E}+1$ & $1.429 E+2$ & $1.189 \mathrm{E}+2$ & $1.486 \mathrm{E}+2$ & $2.713 E+1$ & $2.356 E+5$ & $6.151 \mathrm{E}+3$ & $1.625 \mathrm{E}+1$ \\
\hline \multicolumn{12}{|c|}{ Variant 17} \\
\hline S7 & $2.586 \mathrm{E}+1$ & $8.951 E+0$ & $9.450 \mathrm{E}-1$ & $1.599 \mathrm{E}+1$ & $6.015 E+1$ & $9.892 E+1$ & $6.207 E+1$ & $3.493 E+1$ & $6.657 E+4$ & $2.119 E+3$ & $6.929 E+2$ \\
\hline S8 & $2.506 \mathrm{E}+1$ & $8.821 \mathrm{E}+0$ & $1.007 \mathrm{E}+0$ & $1.545 E+1$ & $5.959 \mathrm{E}+1$ & $1.044 \mathrm{E}+2$ & $6.043 E+1$ & $3.488 \mathrm{E}+1$ & $6.319 E+4$ & $2.011 \mathrm{E}+3$ & $6.584 \mathrm{E}+2$ \\
\hline S9 & $1.255 \mathrm{E}+1$ & $6.689 \mathrm{E}+0$ & $7.143 \mathrm{E}-1$ & $7.033 E+0$ & $1.913 E+1$ & $4.493 \mathrm{E}+1$ & $2.317 E+1$ & $2.638 E+1$ & $8.876 E+3$ & $3.300 \mathrm{E}+2$ & $1.089 E+2$ \\
\hline $\mathrm{S} 10$ & $2.511 \mathrm{E}+1$ & $9.021 \mathrm{E}+0$ & $9.477 \mathrm{E}-1$ & $1.539 \mathrm{E}+1$ & $5.822 \mathrm{E}+1$ & $9.874 \mathrm{E}+1$ & $6.000 \mathrm{E}+1$ & $3.513 E+1$ & $6.223 E+4$ & $1.992 \mathrm{E}+3$ & $6.499 \mathrm{E}+2$ \\
\hline S11 & $2.586 \mathrm{E}+1$ & $8.950 \mathrm{E}+0$ & $9.450 \mathrm{E}-1$ & $1.599 \mathrm{E}+1$ & $6.015 E+1$ & $9.891 \mathrm{E}+1$ & $6.207 E+1$ & $3.492 \mathrm{E}+1$ & $6.656 E+4$ & $2.119 E+3$ & $6.928 \mathrm{E}+2$ \\
\hline $\mathrm{S} 12$ & $3.865 \mathrm{E}+1$ & $9.300 \mathrm{E}+0$ & $9.482 \mathrm{E}-1$ & $2.641 \mathrm{E}+1$ & $8.029 \mathrm{E}+1$ & $1.061 \mathrm{E}+2$ & $8.858 \mathrm{E}+1$ & $3.584 \mathrm{E}+1$ & $1.265 E+5$ & $3.409 \mathrm{E}+3$ & $1.293 E+3$ \\
\hline \multicolumn{12}{|c|}{ Variant 18} \\
\hline S7 & $3.258 \mathrm{E}+1$ & $7.679 \mathrm{E}+0$ & $9.910 \mathrm{E}-1$ & $2.078 \mathrm{E}+1$ & $8.486 \mathrm{E}+1$ & $5.826 \mathrm{E}+1$ & $8.310 \mathrm{E}+1$ & $2.371 \mathrm{E}+1$ & $9.836 \mathrm{E}+4$ & $3.071 \mathrm{E}+3$ & $1.519 \mathrm{E}+1$ \\
\hline S8 & $3.146 \mathrm{E}+1$ & $7.710 \mathrm{E}+0$ & $1.055 \mathrm{E}+0$ & $1.999 \mathrm{E}+1$ & $8.410 \mathrm{E}+1$ & $6.019 \mathrm{E}+1$ & $8.098 \mathrm{E}+1$ & $2.576 \mathrm{E}+1$ & $9.321 E+4$ & $2.910 \mathrm{E}+3$ & $1.497 \mathrm{E}+1$ \\
\hline S9 & $1.410 \mathrm{E}+1$ & $5.929 \mathrm{E}+0$ & 7.548E-1 & $7.925 \mathrm{E}+0$ & $2.509 E+1$ & $2.413 E+1$ & $2.724 E+1$ & $1.700 E+1$ & $1.312 E+4$ & $4.577 \mathrm{E}+2$ & $9.540 \mathrm{E}+0$ \\
\hline S10 & $3.119 \mathrm{E}+1$ & $7.758 \mathrm{E}+0$ & 9.934E-1 & $1.969 E+1$ & $8.131 E+1$ & $5.781 \mathrm{E}+1$ & $7.923 \mathrm{E}+1$ & $2.389 E+1$ & $9.051 \mathrm{E}+4$ & $2.841 E+3$ & $1.502 \mathrm{E}+1$ \\
\hline S11 & $3.258 \mathrm{E}+1$ & $7.681 E+0$ & $9.910 \mathrm{E}-1$ & $2.078 E+1$ & $8.486 \mathrm{E}+1$ & $5.826 \mathrm{E}+1$ & $8.310 \mathrm{E}+1$ & $2.370 \mathrm{E}+1$ & $9.835 E+4$ & $3.071 E+3$ & $1.519 \mathrm{E}+1$ \\
\hline $\mathrm{S} 12$ & $5.105 E+1$ & $7.717 \mathrm{E}+0$ & $1.006 \mathrm{E}+0$ & $3.613 E+1$ & $1.109 \mathrm{E}+2$ & $6.841 E+1$ & $1.192 \mathrm{E}+2$ & $2.168 \mathrm{E}+1$ & $1.846 \mathrm{E}+5$ & $4.824 \mathrm{E}+3$ & $1.883 \mathrm{E}+1$ \\
\hline
\end{tabular}


Table 4.33. Cell relative absorption rates in uranium and plutonium isotopes for Variants 15-18

\begin{tabular}{|c|c|c|c|c|c|c|c|c|c|}
\hline \multirow{2}{*}{ State } & \multicolumn{9}{|c|}{ Cell relative absorption rate } \\
\hline & ${ }^{235} \mathrm{U}$ & ${ }^{236} \mathrm{U}$ & ${ }^{238} \mathrm{U}$ & ${ }^{238} \mathrm{Pu}$ & ${ }^{239} \mathrm{Pu}$ & ${ }^{240} \mathrm{Pu}$ & ${ }^{241} \mathrm{Pu}$ & ${ }^{242} \mathrm{Pu}$ & All FP \\
\hline \multicolumn{10}{|c|}{ Variant 15} \\
\hline s7 & $6.491 \mathrm{E}-1$ & 0.0 & $3.015 \mathrm{E}-1$ & 0.0 & 0.0 & 0.0 & 0.0 & 0.0 & 0.0 \\
\hline S8 & $6.357 \mathrm{E}-1$ & 0.0 & $3.158 \mathrm{E}-1$ & 0.0 & 0.0 & 0.0 & 0.0 & 0.0 & 0.0 \\
\hline S9 & $5.038 \mathrm{E}-1$ & 0.0 & $4.598 \mathrm{E}-1$ & 0.0 & 0.0 & 0.0 & 0.0 & 0.0 & 0.0 \\
\hline S10 & $5.964 \mathrm{E}-1$ & 0.0 & $2.927 \mathrm{E}-1$ & 0.0 & 0.0 & 0.0 & 0.0 & 0.0 & 0.0 \\
\hline S11 & $6.491 \mathrm{E}-1$ & 0.0 & $3.015 \mathrm{E}-1$ & 0.0 & 0.0 & 0.0 & 0.0 & 0.0 & 0.0 \\
\hline $\mathbf{S} 12$ & $6.902 \mathrm{E}-1$ & 0.0 & 2.447E-1 & 0.0 & 0.0 & 0.0 & 0.0 & 0.0 & 0.0 \\
\hline \multicolumn{10}{|c|}{ Variant 16} \\
\hline S7 & $2.429 \mathrm{E}-1$ & $7.329 \mathrm{E}-3$ & $2.849 \mathrm{E}-1$ & $2.815 \mathrm{E}-3$ & $2.502 \mathrm{E}-1$ & $6.169 \mathrm{E}-2$ & $3.845 \mathrm{E}-2$ & $2.113 \mathrm{E}-3$ & $4.321 \mathrm{E}-2$ \\
\hline S8 & 2.337E-1 & $7.709 \mathrm{E}-3$ & $2.983 \mathrm{E}-1$ & $2.692 \mathrm{E}-3$ & $2.476 \mathrm{E}-1$ & $6.400 \mathrm{E}-2$ & $3.737 \mathrm{E}-2$ & $2.176 \mathrm{E}-3$ & $4.220 \mathrm{E}-2$ \\
\hline S9 & $1.904 \mathrm{E}-1$ & $1.137 \mathrm{E}-2$ & $4.463 \mathrm{E}-1$ & $1.962 \mathrm{E}-3$ & $1.524 \mathrm{E}-1$ & $5.617 \mathrm{E}-2$ & $2.396 \mathrm{E}-2$ & $3.251 \mathrm{E}-3$ & $5.296 \mathrm{E}-2$ \\
\hline S10 & 2.237E-1 & $7.231 \mathrm{E}-3$ & $2.781 \mathrm{E}-1$ & $2.563 \mathrm{E}-3$ & $2.318 \mathrm{E}-1$ & $6.008 \mathrm{E}-2$ & 3.533E-2 & $2.085 \mathrm{E}-3$ & 4.138E-2 \\
\hline S11 & 2.429E-1 & $7.330 \mathrm{E}-3$ & $2.849 \mathrm{E}-1$ & $2.815 \mathrm{E}-3$ & $2.502 \mathrm{E}-1$ & $6.169 \mathrm{E}-2$ & $3.845 \mathrm{E}-2$ & $2.113 \mathrm{E}-3$ & $4.321 \mathrm{E}-2$ \\
\hline S12 & $2.936 \mathrm{E}-1$ & $5.547 \mathrm{E}-3$ & $2.365 \mathrm{E}-1$ & $3.756 \mathrm{E}-3$ & $2.366 \mathrm{E}-1$ & 5.437E-2 & $4.071 \mathrm{E}-2$ & $1.630 \mathrm{E}-3$ & 4.297E-2 \\
\hline \multicolumn{10}{|c|}{ Variant 17} \\
\hline S7 & $1.521 \mathrm{E}-2$ & 0.0 & $2.739 \mathrm{E}-1$ & 0.0 & $6.072 \mathrm{E}-1$ & $6.415 \mathrm{E}-2$ & $6.682 \mathrm{E}-3$ & 0.0 & 0.0 \\
\hline S8 & $1.453 \mathrm{E}-2$ & 0.0 & $2.876 \mathrm{E}-1$ & 0.0 & $5.928 \mathrm{E}-1$ & $6.675 \mathrm{E}-2$ & $6.411 \mathrm{E}-3$ & 0.0 & 0.0 \\
\hline S9 & $1.624 \mathrm{E}-2$ & 0.0 & $4.556 \mathrm{E}-1$ & 0.0 & $4.248 \mathrm{E}-1$ & $6.411 \mathrm{E}-2$ & $5.488 \mathrm{E}-3$ & 0.0 & 0.0 \\
\hline S10 & $1.454 \mathrm{E}-2$ & 0.0 & 2.705E-1 & 0.0 & 5.787E-1 & $6.305 \mathrm{E}-2$ & $6.359 \mathrm{E}-3$ & 0.0 & 0.0 \\
\hline S11 & $1.521 \mathrm{E}-2$ & 0.0 & $2.739 \mathrm{E}-1$ & 0.0 & $6.072 \mathrm{E}-1$ & $6.415 \mathrm{E}-2$ & $6.682 \mathrm{E}-3$ & 0.0 & 0.0 \\
\hline S12 & $1.831 \mathrm{E}-2$ & 0.0 & $2.213 \mathrm{E}-1$ & 0.0 & $6.525 \mathrm{E}-1$ & 5.541E-2 & 7.677E-3 & 0.0 & 0.0 \\
\hline \multicolumn{10}{|c|}{ Variant 18} \\
\hline S7 & $9.801 \mathrm{E}-3$ & $4.105 \mathrm{E}-4$ & $2.756 \mathrm{E}-1$ & $3.497 \mathrm{E}-4$ & $4.111 \mathrm{E}-1$ & $1.284 \mathrm{E}-1$ & $9.515 \mathrm{E}-2$ & $6.172 \mathrm{E}-3$ & $2.396 \mathrm{E}-2$ \\
\hline S8 & $9.327 \mathrm{E}-3$ & $4.062 \mathrm{E}-4$ & 2.892E-1 & $3.314 \mathrm{E}-4$ & $4.015 \mathrm{E}-1$ & $1.307 \mathrm{E}-1$ & $9.138 \mathrm{E}-2$ & $6.610 \mathrm{E}-3$ & $2.327 \mathrm{E}-2$ \\
\hline S9 & $9.172 E-3$ & $6.851 \mathrm{E}-4$ & $4.538 \mathrm{E}-1$ & $2.883 \mathrm{E}-4$ & $2.628 \mathrm{E}-1$ & $1.150 \mathrm{E}-1$ & $6.741 \mathrm{E}-2$ & $9.567 \mathrm{E}-3$ & $3.253 \mathrm{E}-2$ \\
\hline S10 & $9.206 \mathrm{E}-3$ & $4.069 \mathrm{E}-4$ & 2.711E-1 & $3.250 \mathrm{E}-4$ & $3.865 \mathrm{E}-1$ & $1.250 \mathrm{E}-1$ & 8.901E-2 & $6.102 E-3$ & $2.325 \mathrm{E}-2$ \\
\hline S11 & $9.801 \mathrm{E}-3$ & $4.106 \mathrm{E}-4$ & $2.756 \mathrm{E}-1$ & $3.497 \mathrm{E}-4$ & $4.111 \mathrm{E}-1$ & $1.284 \mathrm{E}-1$ & $9.515 \mathrm{E}-2$ & $6.171 \mathrm{E}-3$ & $2.396 \mathrm{E}-2$ \\
\hline S12 & $1.242 \mathrm{E}-2$ & $3.336 \mathrm{E}-4$ & $2.264 \mathrm{E}-1$ & $4.916 \mathrm{E}-4$ & $4: 345 \mathrm{E}-1$ & $1.219 \mathrm{E}-1$ & $1.104 \mathrm{E}-1$ & $4.564 \mathrm{E}-3$ & $2.402 \mathrm{E}-2$ \\
\hline
\end{tabular}


Table 4.34. Delayed neutron fraction $(\beta)$, effective delayed neutron fraction $\left(\beta_{\mathrm{eff}}\right)$, and prompt neutron lifetime for Variants 15-18

\begin{tabular}{|c|c|c|c|c|}
\hline Variant & State & $\begin{array}{c}\text { Delayed neutron } \\
\text { fraction }\end{array}$ & $\begin{array}{c}\text { Effective delayed } \\
\text { neutron fraction }\end{array}$ & $\begin{array}{l}\text { Prompt neutron } \\
\text { lifetime (s) }\end{array}$ \\
\hline V15 & $\begin{array}{l}\text { S7 } \\
\text { S8 } \\
\text { S9 } \\
\text { S10 } \\
\text { S11 } \\
\text { S12. }\end{array}$ & $\begin{array}{l}7.374 \mathrm{E}-3 \\
7.386 \mathrm{E}-3 \\
8.153 \mathrm{E}-3 \\
7.430 \mathrm{E}-3 \\
7.457 \mathrm{E}-3 \\
7.304 \mathrm{E}-3\end{array}$ & $\begin{array}{l}7.192 \mathrm{E}-3 \\
7.194 \mathrm{E}-3 \\
7.500 \mathrm{E}-3 \\
7.202 \mathrm{E}-3 \\
8.225 \mathrm{E}-3 \\
8.354 \mathrm{E}-3\end{array}$ & $\begin{array}{l}2.111 \mathrm{E}-5 \\
2.106 \mathrm{E}-5 \\
1.591 \mathrm{E}-5 \\
1.881 \mathrm{E}-5 \\
2.035 \mathrm{E}-5 \\
2.089 \mathrm{E}-5\end{array}$ \\
\hline V16 & $\begin{array}{l}\text { S7 } \\
\text { S8 } \\
\text { S9 } \\
\text { S10 } \\
\text { S11 } \\
\text { S12 }\end{array}$ & $\begin{array}{l}5.515 \mathrm{E}-3 \\
5.515 \mathrm{E}-3 \\
6.991 \mathrm{E}-3 \\
5.585 \mathrm{E}-3 \\
5.551 \mathrm{E}-3 \\
5.554 \mathrm{E}-3\end{array}$ & $\begin{array}{l}5.289 \mathrm{E}-3 \\
5.276 \mathrm{E}-3 \\
6.061 \mathrm{E}-3 \\
5.317 \mathrm{E}-3 \\
5.558 \mathrm{E}-3 \\
5.846 \mathrm{E}-3\end{array}$ & $\begin{array}{l}1.909 \mathrm{E}-5 \\
1.876 \mathrm{E}-5 \\
1.253 \mathrm{E}-5 \\
1.692 \mathrm{E}-5 \\
1.888 \mathrm{E}-5 \\
2.159 \mathrm{E}-5\end{array}$ \\
\hline V17 & $\begin{array}{l}\text { S7 } \\
\text { S8 } \\
\text { S9 } \\
\text { S10 } \\
\text { S11 } \\
\text { S12 }\end{array}$ & $\begin{array}{l}3.353 \mathrm{E}-3 \\
3.378 \mathrm{E}-3 \\
4.691 \mathrm{E}-3 \\
3.402 \mathrm{E}-3 \\
3.442 \mathrm{E}-3 \\
3.198 \mathrm{E}-3\end{array}$ & $\begin{array}{l}3.231 \mathrm{E}-3 \\
3.248 \mathrm{E}-3 \\
4.160 \mathrm{E}-3 \\
3.266 \mathrm{E}-3 \\
3.657 \mathrm{E}-3 \\
3.583 \mathrm{E}-3\end{array}$ & $\begin{array}{l}1.088 \mathrm{E}-5 \\
1.057 \mathrm{E}-5 \\
8.483 \mathrm{E}-6 \\
1.011 \mathrm{E}-5 \\
1.065 \mathrm{E}-5 \\
1.303 \mathrm{E}-5\end{array}$ \\
\hline V18 & $\begin{array}{l}\text { S7 } \\
\text { S8 } \\
\text { S9 } \\
\text { S10 } \\
\text { S11 } \\
\text { S12 }\end{array}$ & $\begin{array}{l}4.085 \mathrm{E}-3 \\
4.109 \mathrm{E}-3 \\
5.711 \mathrm{E}-3 \\
4.153 \mathrm{E}-3 \\
4.113 \mathrm{E}-3 \\
3.886 \mathrm{E}-3\end{array}$ & $\begin{array}{l}3.896 \mathrm{E}-3 \\
3.908 \mathrm{E}-3 \\
4.880 \mathrm{E}-3 \\
3.938 \mathrm{E}-3 \\
4.055 \mathrm{E}-3 \\
4.046 \mathrm{E}-3\end{array}$ & $\begin{array}{l}1.427 \mathrm{E}-5 \\
1.388 \mathrm{E}-5 \\
9.493 \mathrm{E}-6 \\
1.292 \mathrm{E}-5 \\
1.417 \mathrm{E}-5 \\
1.739 \mathrm{E}-5\end{array}$ \\
\hline V19 & $\begin{array}{l}\text { S7 } \\
\text { S8 } \\
\text { S9 } \\
\text { S10 } \\
\text { S11 } \\
\text { S12 }\end{array}$ & $\begin{array}{l}6.338 \mathrm{E}-3 \\
6.354 \mathrm{E}-3 \\
7.201 \mathrm{E}-3 \\
6.366 \mathrm{E}-3 \\
6.425 \mathrm{E}-3 \\
6.279 \mathrm{E}-3\end{array}$ & $\begin{array}{l}6.172 E-3 \\
6.179 E-3 \\
6.596 \mathrm{E}-3 \\
6.161 \mathrm{E}-3 \\
7.083 \mathrm{E}-3 \\
7.191 \mathrm{E}-3\end{array}$ & $\begin{array}{l}1.943 \mathrm{E}-5 \\
1.933 \mathrm{E}-5 \\
1.486 \mathrm{E}-5 \\
1.726 \mathrm{E}-5 \\
1.878 \mathrm{E}-5 \\
1.987 \mathrm{E}-5\end{array}$ \\
\hline V20 & $\begin{array}{l}\text { S7 } \\
\text { S8 } \\
\text { S9 } \\
\text { S10 } \\
\text { S11 } \\
\text { S12 } \\
\end{array}$ & $\begin{array}{l}4.833 \mathrm{E}-3 \\
4.838 \mathrm{E}-3 \\
6.206 \mathrm{E}-3 \\
4.884 \mathrm{E}-3 \\
4.887 \mathrm{E}-3 \\
4.815 \mathrm{E}-3\end{array}$ & $\begin{array}{l}4.650 \mathrm{E}-3 \\
4.645 \mathrm{E}-3 \\
5.453 \mathrm{E}-3 \\
4.667 \mathrm{E}-3 \\
5.027 \mathrm{E}-3 \\
5.198 \mathrm{E}-3\end{array}$ & $\begin{array}{l}1.772 \mathrm{E}-5 \\
1.742 \mathrm{E}-5 \\
1.239 \mathrm{E}-5 \\
1.570 \mathrm{E}-5 \\
1.744 \mathrm{E}-5 \\
2.010 \mathrm{E}-5\end{array}$ \\
\hline
\end{tabular}




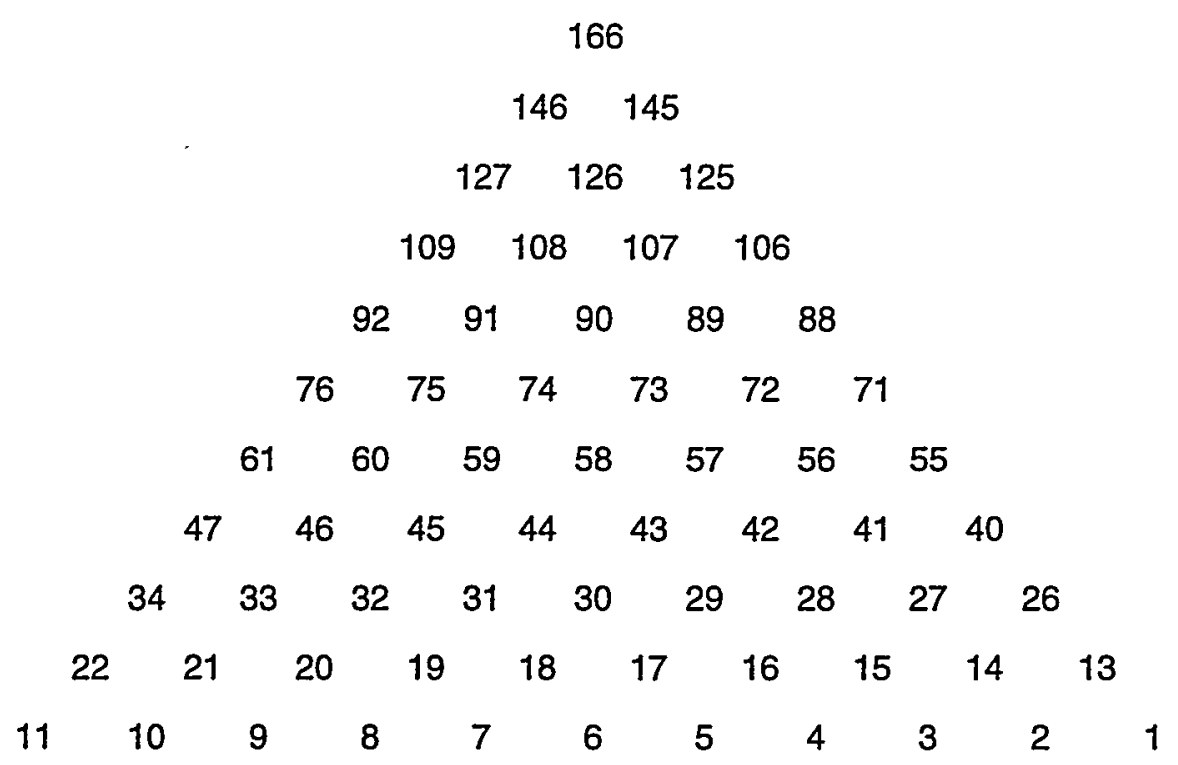

Fig. 4.1. Pin numbering for Variants 11 and 12.

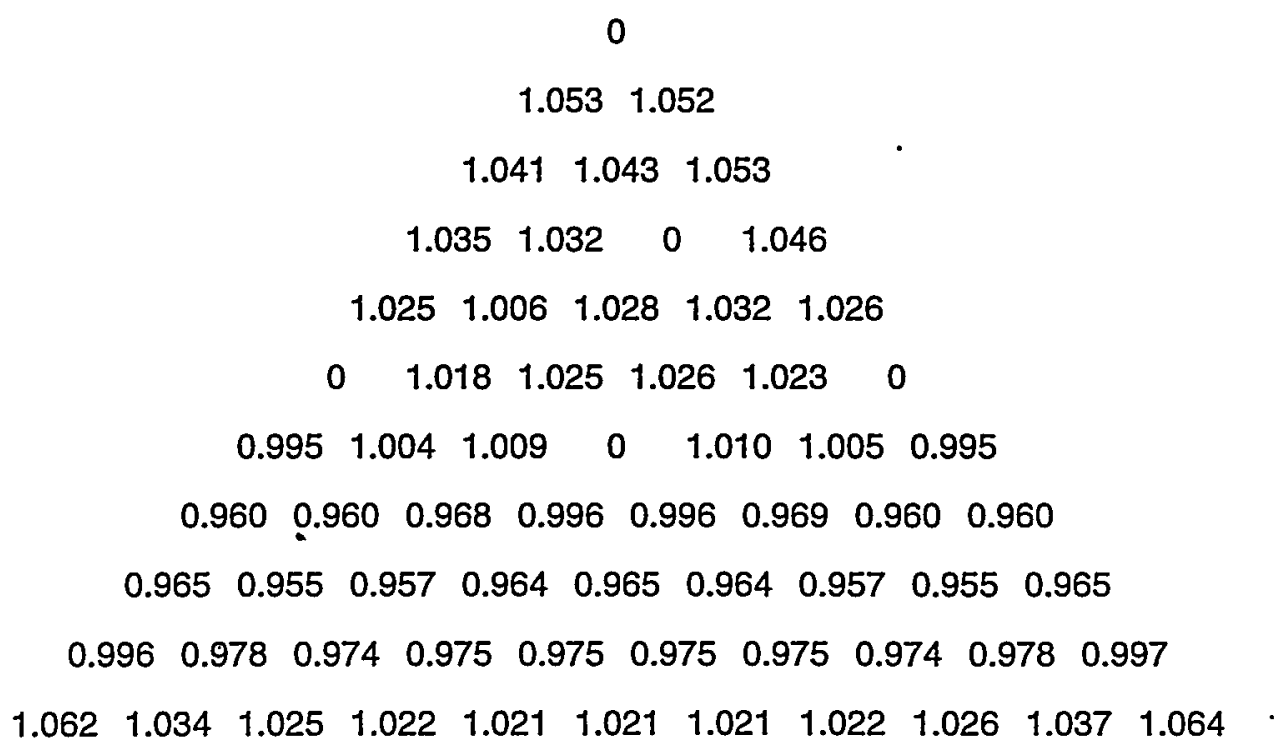

Fig. 4.2. Relative fission rate distribution for Variant 11 , State 1 , at $0 \mathrm{MWd} / \mathrm{kg}$. 


$$
\begin{aligned}
& 0 \\
& 1.0501 .049 \\
& 1.037 \quad 1.040 \quad 1.053 \\
& \begin{array}{llll}
1.031 & 1.030 & 0 & 1.048
\end{array} \\
& \begin{array}{lllll}
1.024 & 1.004 & 1.027 & 1.030 & 1.025
\end{array} \\
& \begin{array}{llllll}
0 & 1.017 & 1.023 & 1.024 & 1.021 & 0
\end{array} \\
& \begin{array}{lllllll}
0.997 & 1.004 & 1.009 & 0 & 1.010 & 1.005 & 0.997
\end{array} \\
& \begin{array}{llllllll}
0.964 & 0.962 & 0.970 & 0.997 & 0.997 & 0.971 & 0.962 & 0.964
\end{array} \\
& \begin{array}{lllllllll}
0.968 & 0.958 & 0.960 & 0.966 & 0.967 & 0.966 & 0.960 & 0.958 & 0.968
\end{array} \\
& \begin{array}{llllllllll}
0.996 & 0.978 & 0.975 & 0.975 & 0.976 & 0.976 & 0.975 & 0.975 & 0.978 & 0.997
\end{array}
\end{aligned}
$$

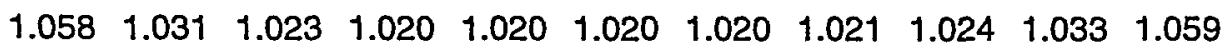

Fig. 4.3. Relative fission rate distribution for Variant 11, State 1 , at $10 \mathrm{MWd} / \mathrm{kg}$.

$$
\begin{aligned}
& 0 \\
& 1.0441 .043 \\
& 1.0311 .0351 .049 \\
& \begin{array}{llll}
1.026 & 1.027 & 0 & 1.044
\end{array} \\
& \begin{array}{lllll}
1.022 & 1.003 & 1.023 & 1.026 & 1.023
\end{array} \\
& \begin{array}{llllll}
0 & 1.015 & 1.020 & 1.021 & 1.018 & 0
\end{array} \\
& \begin{array}{lllllll}
0.999 & 1.004 & 1.008 & 0 & 1.009 & 1.005 & 0.999
\end{array} \\
& \begin{array}{llllllll}
0.969 & 0.967 & 0.974 & 0.998 & 0.998 & 0.974 & 0.967 & 0.969
\end{array} \\
& \begin{array}{lllllllll}
0.974 & 0.963 & 0.965 & 0.970 & 0.971 & 0.970 & 0.965 & 0.963 & 0.974
\end{array} \\
& \begin{array}{llllllllll}
0.996 & 0.980 & 0.977 & 0.977 & 0.978 & 0.978 & 0.978 & 0.977 & 0.980 & 0.997
\end{array} \\
& \begin{array}{lllllllllll}
1.050 & 1.026 & 1.020 & 1.018 & 1.017 & 1.017 & 1.018 & 1.018 & 1.021 & 1.028 & 1.051
\end{array}
\end{aligned}
$$

Fig. 4.4. Relative fission rate distribution for Variant 11, State 1 , at $20 \mathrm{MWd} / \mathrm{kg}$. 


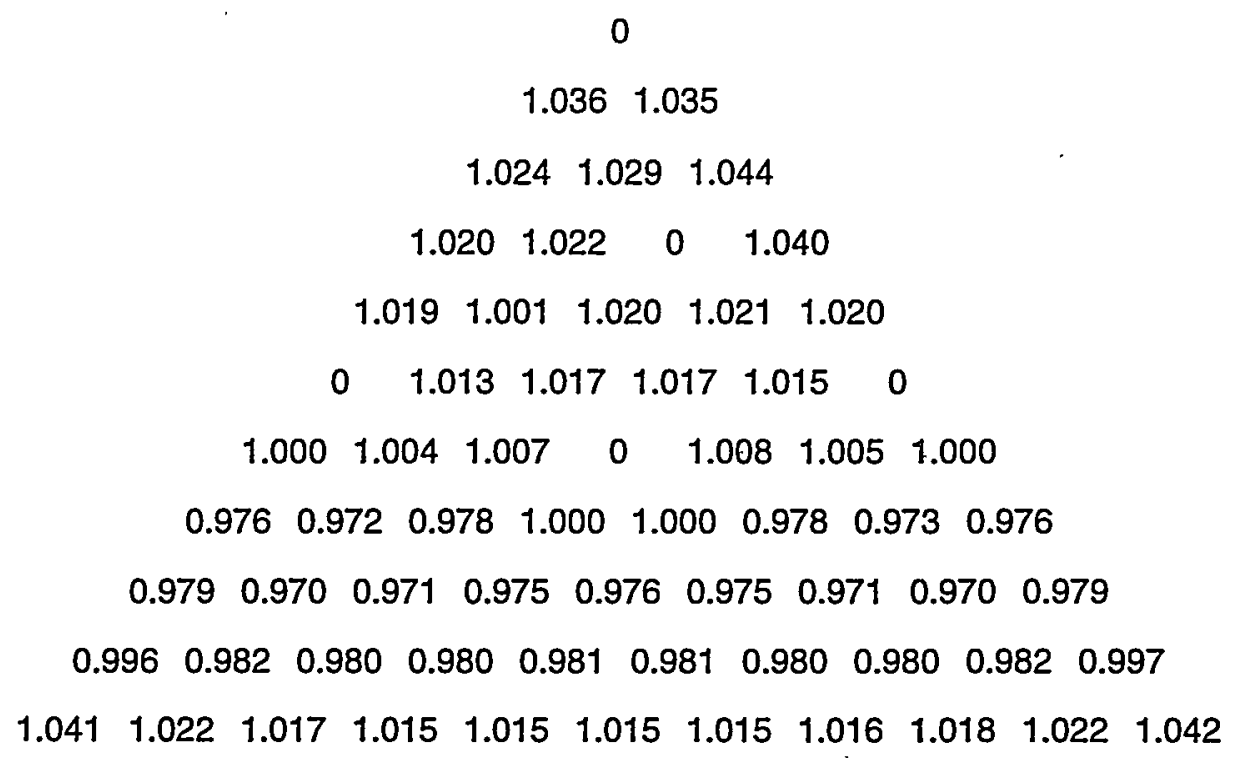

Fig. 4.5. Relative fission rate distribution for Variant 11, State 1 , at $30 \mathrm{MWd} / \mathrm{kg}$.

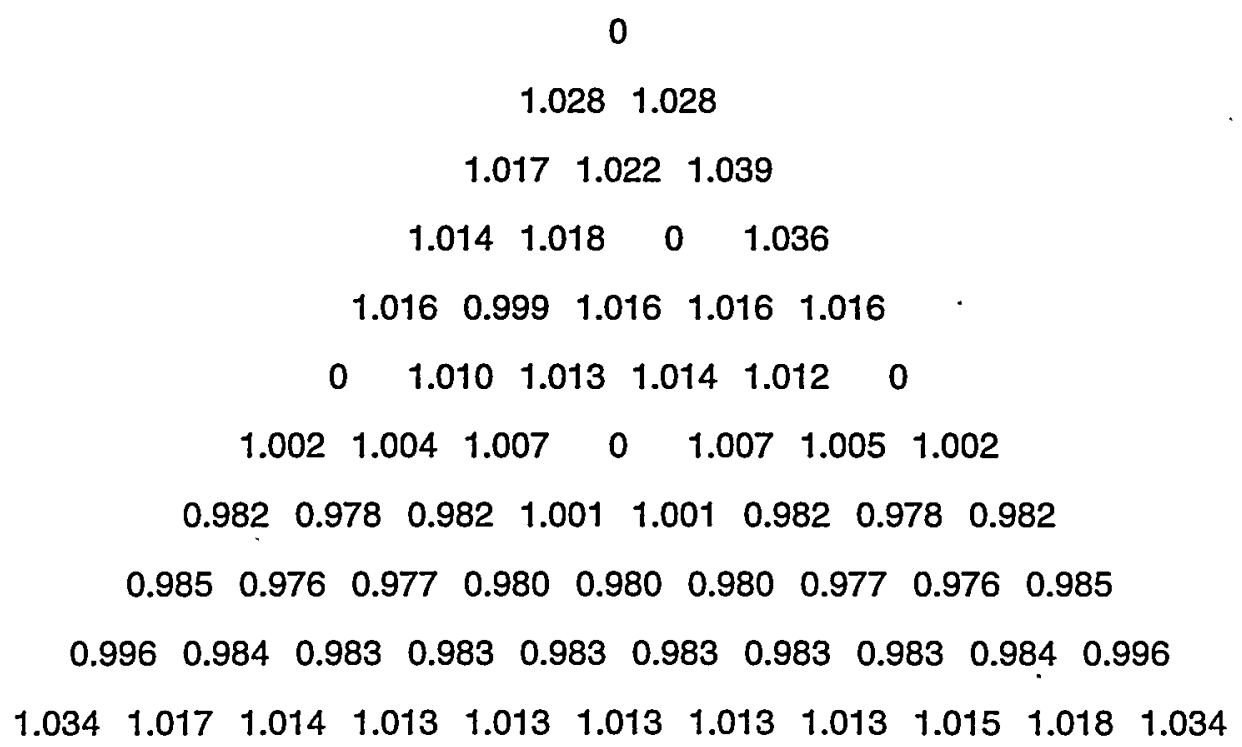

Fig. 4.6. Relative fission rate distribution for Variant 11 , State 1 , at $40 \mathrm{MWd} / \mathrm{kg}$. 


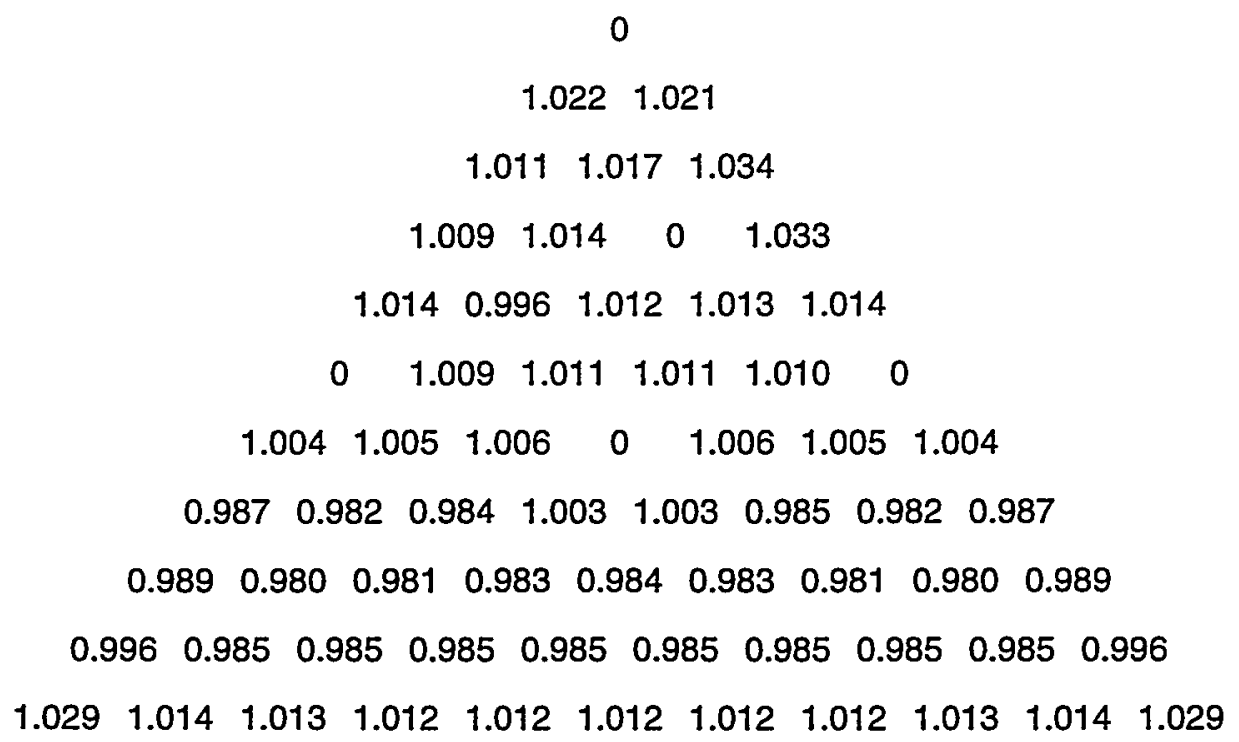

Fig. 4.7. Relative fission rate distribution for Variant 11, State 1 , at $50 \mathrm{MWd} / \mathrm{kg}$.

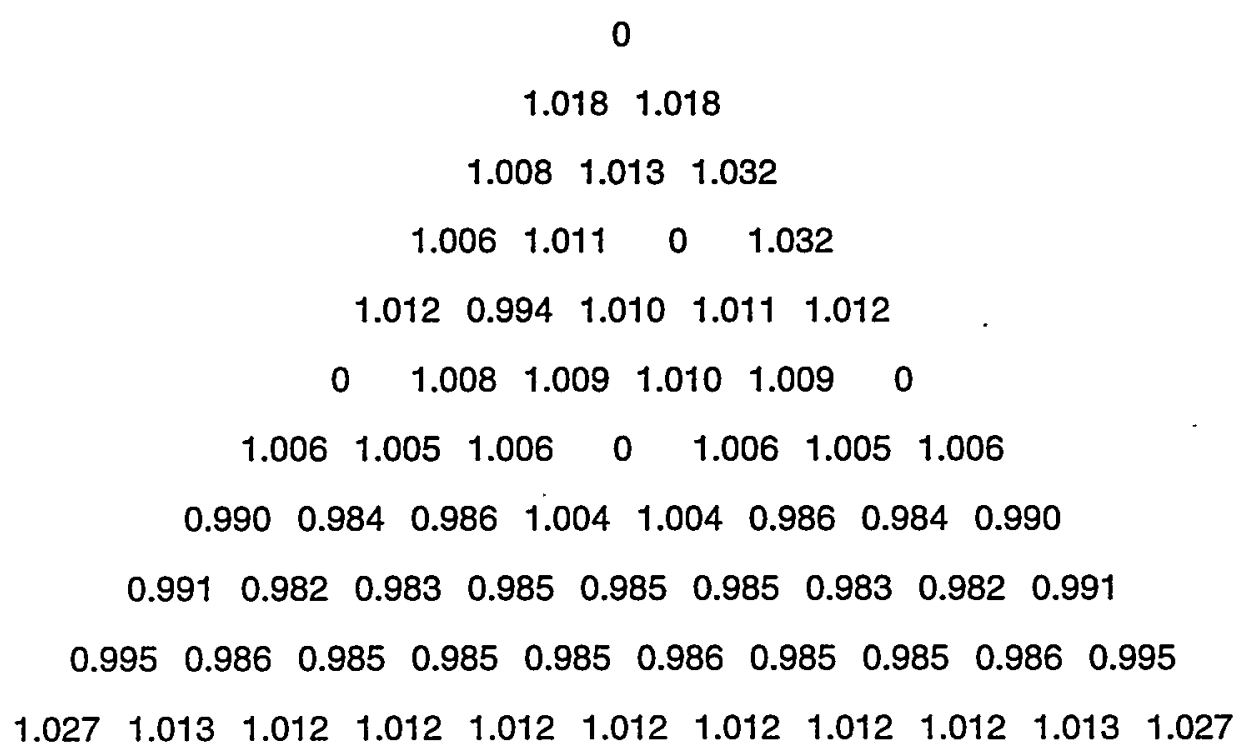

Fig. 4.8. Relative fission rate distribution for Variant 11, State 1 , at $60 \mathrm{MWd} / \mathrm{kg}$. 


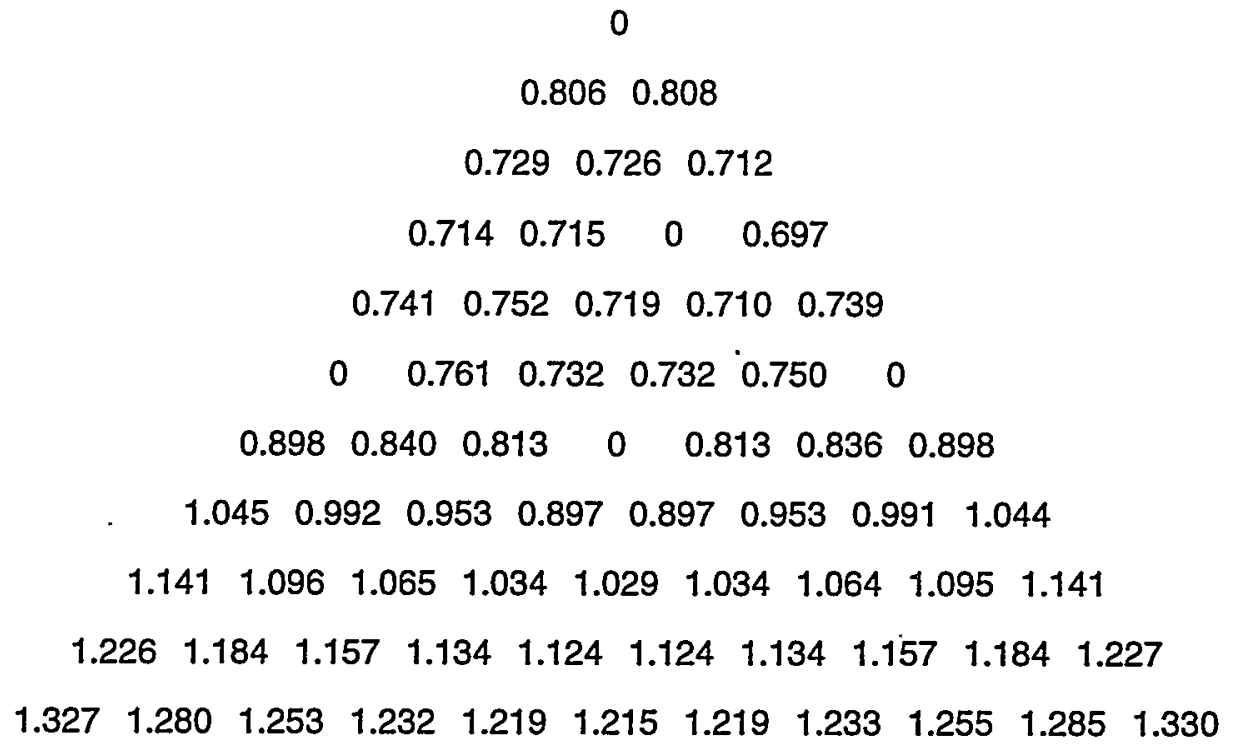

Fig. 4.9. Relative fission rate distribution for Variant 11, State 2, at $0 \mathrm{MWd} / \mathrm{kg}$.

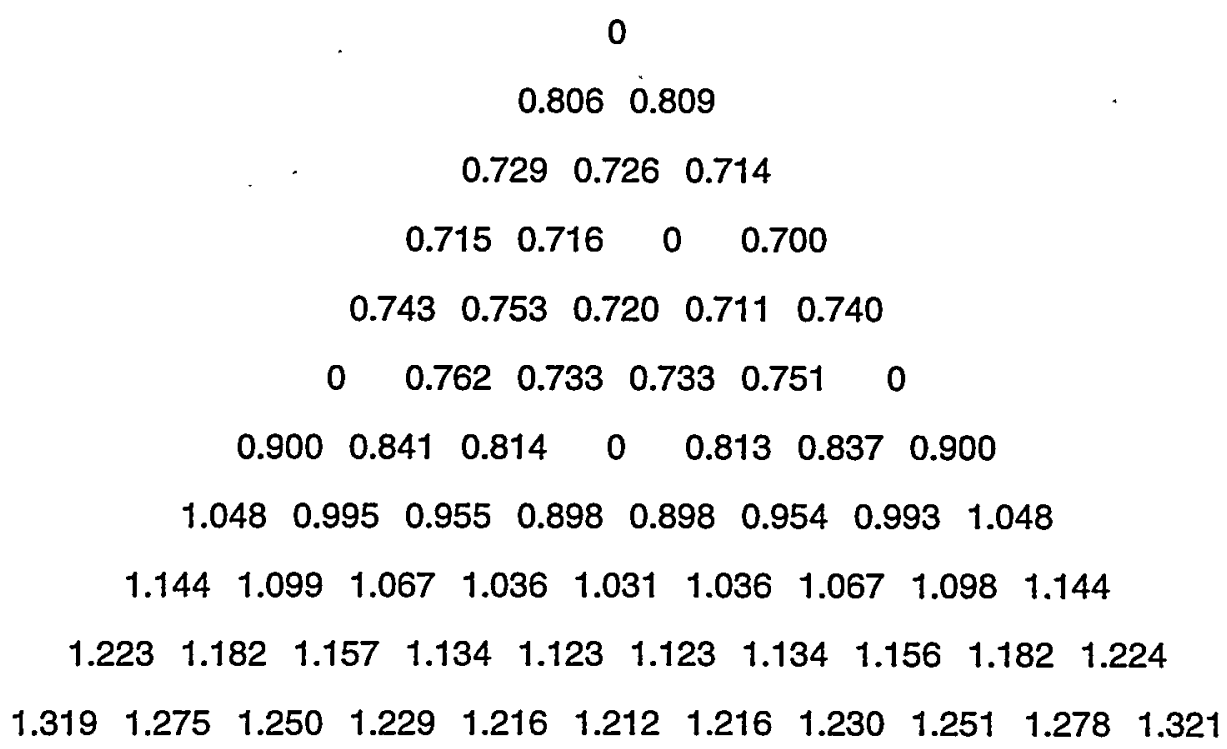

Fig. 4.10. Relative fission rate distribution for Variant 11, State 2, at $10 \mathrm{MWd} / \mathrm{kg}$. 


$$
\begin{aligned}
& 0 \\
& 0.8020 .804 \\
& \begin{array}{lll}
0.725 & 0.723 & 0.712
\end{array} \\
& \begin{array}{llll}
0.711 & 0.714 & 0 & 0.698
\end{array} \\
& \begin{array}{lllll}
0.741 & 0.752 & 0.718 & 0.708 & 0.738
\end{array} \\
& \begin{array}{llllll}
0 & 0.761 & 0.731 & 0.731 & 0.748 & 0
\end{array} \\
& \begin{array}{lllllll}
0.901 & 0.840 & 0.813 & 0 & 0.812 & 0.836 & 0.900
\end{array} \\
& \begin{array}{llllllll}
1.054 & 0.999 & 0.958 & 0.899 & 0.899 & 0.957 & 0.997 & 1.054
\end{array} \\
& \begin{array}{lllllllll}
1.150 & 1.104 & 1.073 & 1.040 & 1.035 & 1.040 & 1.073 & 1.104 & 1.150
\end{array} \\
& \begin{array}{llllllllll}
1.223 & 1.184 & 1.159 & 1.136 & 1.126 & 1.126 & 1.136 & 1.159 & 1.184 & 1.223
\end{array}
\end{aligned}
$$

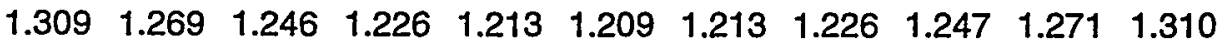

Fig. 4.11. Relative fission rate distribution for Variant 11, State 2, at $20 \mathrm{MWd} / \mathrm{kg}$.

$$
\begin{aligned}
& 0 \\
& 0.795 \quad 0.797 \\
& \begin{array}{lll}
0.719 & 0.718 & 0.708
\end{array} \\
& \begin{array}{llll}
0.706 & 0.710 & 0 & 0.694
\end{array} \\
& \begin{array}{lllll}
0.738 & 0.749 & 0.714 & 0.704 & 0.735
\end{array} \\
& \begin{array}{llllll}
0 & 0.758 & 0.728 & 0.728 & 0.745 & 0
\end{array}
\end{aligned}
$$

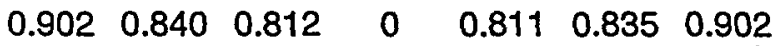

$$
\begin{aligned}
& \begin{array}{llllllll}
1.061 & 1.004 & 0.961 & 0.900 & 0.900 & 0.960 & 1.002 & 1.061
\end{array} \\
& \begin{array}{lllllllll}
1.157 & 1.112 & 1.079 & 1.045 & 1.039 & 1.045 & 1.079 & 1.111 & 1.157
\end{array}
\end{aligned}
$$

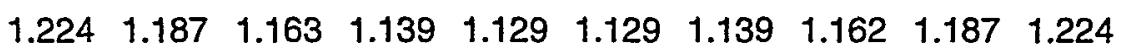

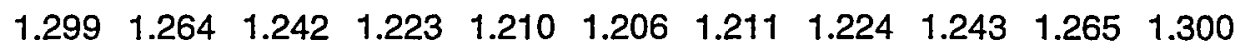

Fig. 4.12. Relative fission rate distribution for Variant 11, State 2, at $30 \mathrm{MWd} / \mathrm{kg}$. 


$$
\begin{aligned}
& 0 \\
& 0.7860 .788 \\
& \begin{array}{lll}
0.712 & 0.712 & 0.702
\end{array} \\
& \begin{array}{llll}
0.700 & 0.705 & 0 & 0.690
\end{array} \\
& \begin{array}{lllll}
0.734 & 0.745 & 0.710 & 0.700 & 0.731
\end{array} \\
& \begin{array}{llllll}
0 & 0.755 & 0.724 & 0.724 & 0.742 & 0
\end{array} \\
& \begin{array}{lllllll}
0.903 & 0.839 & 0.810 & 0 & 0.809 & 0.835 & 0.903
\end{array}
\end{aligned}
$$

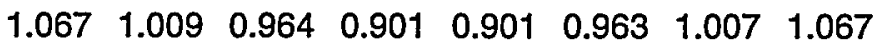

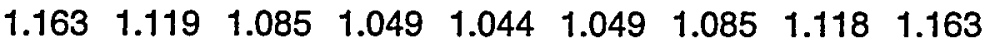

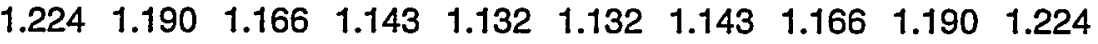

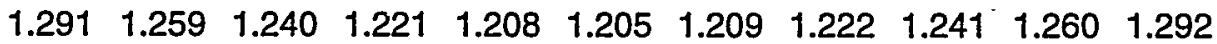

Fig. 4.13. Relative fission rate distribution for Variant 11, State 2, at $40 \mathrm{MWd} / \mathrm{kg}$.

$$
\begin{aligned}
& 0 \\
& 0.7780 .780 \\
& 0.706 \quad 0.706 \quad 0.698 \\
& \begin{array}{llll}
0.695 & 0.700 & 0 & 0.686
\end{array} \\
& \begin{array}{lllll}
0.731 & 0.741 & 0.706 & 0.696 & 0.728
\end{array} \\
& \begin{array}{llllll}
0 & 0.753 & 0.721 & 0.721 & 0.740 & 0
\end{array} \\
& \begin{array}{lllllll}
0.905 & 0.839 & 0.810 & 0 & 0.809 & 0.835 & 0.904
\end{array} \\
& \begin{array}{llllllll}
1.072 & 1.012 & 0.966 & 0.902 & 0.902 & 0.965 & 1.010 & 1.072
\end{array} \\
& \begin{array}{lllllllll}
1.168 & 1.124 & 1.090 & 1.053 & 1.047 & 1.052 & 1.089 & 1.123 & 1.168
\end{array}
\end{aligned}
$$

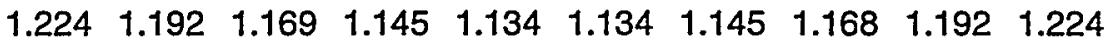

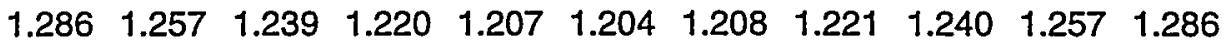

Fig. 4.14. Relative fission rate distribution for Variant 11, State 2 , at $50 \mathrm{MWd} / \mathrm{kg}$. 


$$
\begin{aligned}
& 0 \\
& 0.773 \quad 0.776 \\
& 0.703 \quad 0.703 \quad 0.696 \\
& \begin{array}{llll}
0.692 & 0.698 & 0 & 0.685
\end{array} \\
& \begin{array}{lllll}
0.729 & 0.738 & 0.704 & 0.694 & 0.726
\end{array} \\
& \begin{array}{llllll}
0 & 0.752 & 0.720 & 0.720 & 0.739 & 0
\end{array} \\
& \begin{array}{lllllll}
0.906 & 0.840 & 0.810 & 0 & 0.809 & 0.835 & 0.906
\end{array} \\
& \begin{array}{llllllll}
1.074 & 1.014 & 0.966 & 0.904 & 0.903 & 0.965 & 1.012 & 1.074
\end{array}
\end{aligned}
$$

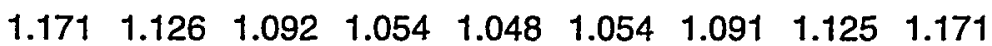

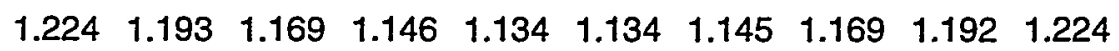

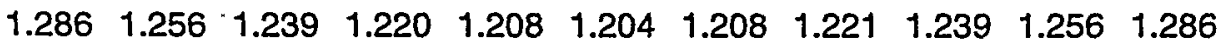

Fig. 4.15. Relative fission rate distribution for Variant 11, State 2, at $60 \mathrm{MWd} / \mathrm{kg}$.

$$
\begin{aligned}
& 0 \\
& 1.0701 .069 \\
& 1.0471 .0551 .082 \\
& \begin{array}{llll}
1.038 & 1.041 \quad 0 & 1.075
\end{array} \\
& \begin{array}{lllll}
1.036 & 0.993 & 1.039 & 1.044 & 1.039
\end{array} \\
& \begin{array}{llllll}
0 & 1.028 & 1.036 & 1.038 & 1.035 & 0
\end{array}
\end{aligned}
$$

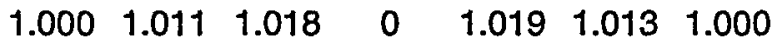

$$
\begin{aligned}
& \begin{array}{llllllll}
0.940 & 0.937 & 0.949 & 1.001 & 1.001 & 0.949 & 0.937 & 0.940
\end{array}
\end{aligned}
$$

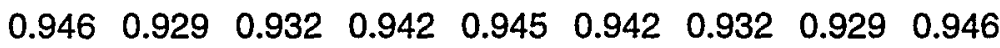

$$
\begin{aligned}
& \begin{array}{llllllllll}
0.989 & 0.960 & 0.956 & 0.957 & 0.959 & 0.959 & 0.958 & 0.956 & 0.961 & 0.991
\end{array} \\
& \begin{array}{lllllllllll}
1.105 & 1.057 & 1.046 & 1.043 & 1.043 & 1.043 & 1.043 & 1.044 & 1.048 & 1.061 & 1.108
\end{array}
\end{aligned}
$$

Fig. 4.16. Relative fission rate distribution for Variant 12, State 1 , at $0 \mathrm{MWd} / \mathrm{kg}$. 
0

1.0591 .058

$1.0421 .047 \quad 1.067$

$\begin{array}{llll}1.035 & 1.036 & 0 & 1.062\end{array}$

$\begin{array}{lllll}1.031 & 0.999 & 1.033 & 1.037 & 1.032\end{array}$

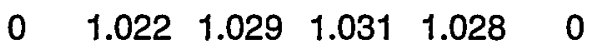

$\begin{array}{lllllll}0.999 & 1.008 & 1.014 & 0 & 1.015 & 1.009 & 0.999\end{array}$

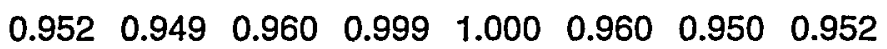

$\begin{array}{lllllllll}0.958 & 0.943 & 0.946 & 0.954 & 0.956 & 0.954 & 0.946 & 0.943 & 0.958\end{array}$

$\begin{array}{llllllllll}0.994 & 0.969 & 0.965 & 0.966 & 0.967 & 0.967 & 0.966 & 0.965 & 0.970 & 0.995\end{array}$

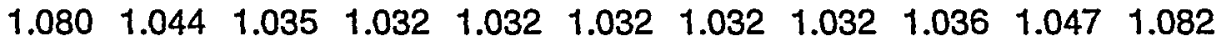

Fig. 4.17. Relative fission rate distribution for Variant 12, State 1 , at $10 \mathrm{MWd} / \mathrm{kg}$.

\author{
0 \\ $1.048 \quad 1.047$ \\ 1.0341 .0391 .056 \\ $\begin{array}{llll}1.028 & 1.030 \quad 0 & 1.050\end{array}$ \\ $\begin{array}{lllll}1.025 & 1.003 & 1.027 & 1.029 & 1.026\end{array}$

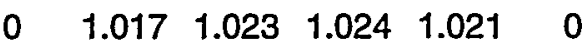 \\ $\begin{array}{lllllll}0.999 & 1.005 & 1.010 & 0 & 1.010 & 1.006 & 0.999\end{array}$ \\ $\begin{array}{llllllll}0.964 & 0.961 & 0.969 & 0.999 & 0.999 & 0.970 & 0.962 & 0.964\end{array}$ \\ $\begin{array}{lllllllll}0.970 & 0.957 & 0.959 & 0.965 & 0.967 & 0.965 & 0.959 & 0.957 & 0.970\end{array}$ \\ $\begin{array}{llllllllll}0.997 & 0.977 & 0.974 & 0.974 & 0.975 & 0.975 & 0.974 & 0.974 & 0.977 & 0.997\end{array}$

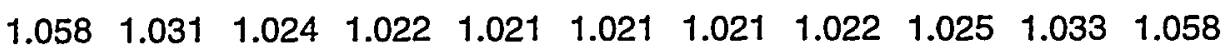

Fig. 4.18. Relative fission rate distribution for Variant 12, State 1 , at $20 \mathrm{MWd} / \mathrm{kg}$. 


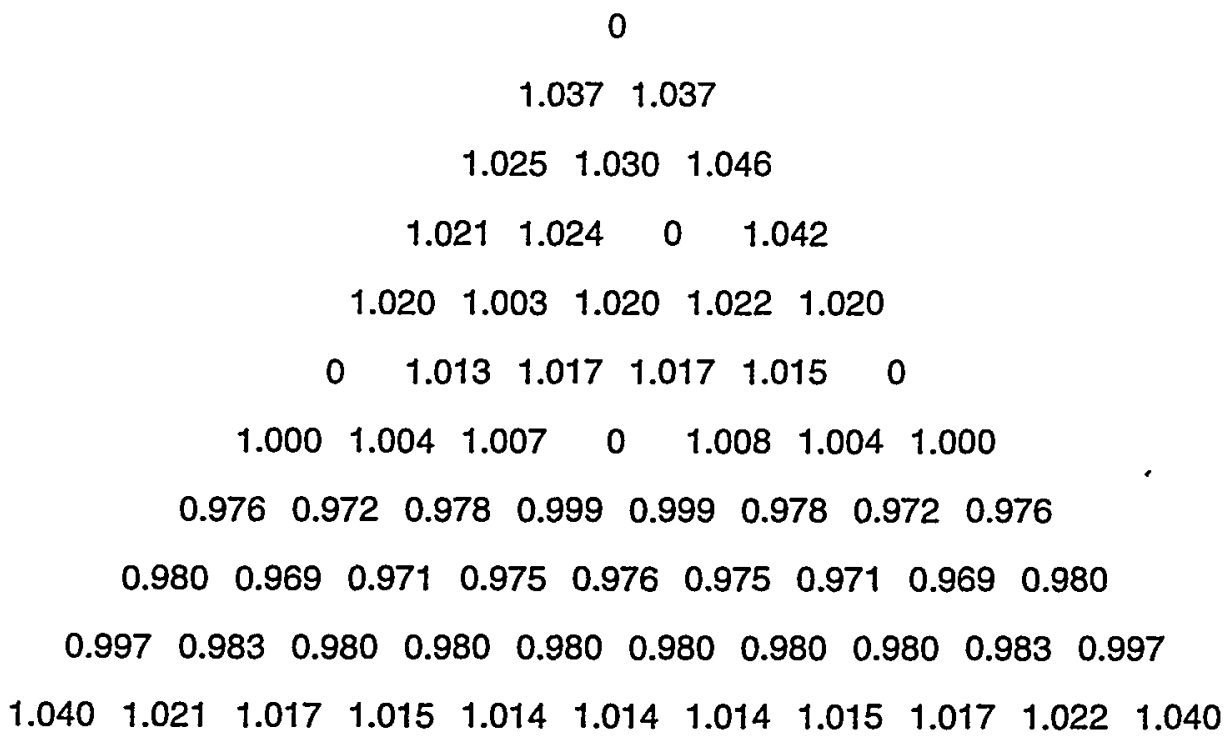

Fig. 4.19. Relative fission rate distribution for Variant 12 , State 1 , at $30 \mathrm{MWd} / \mathrm{kg}$.

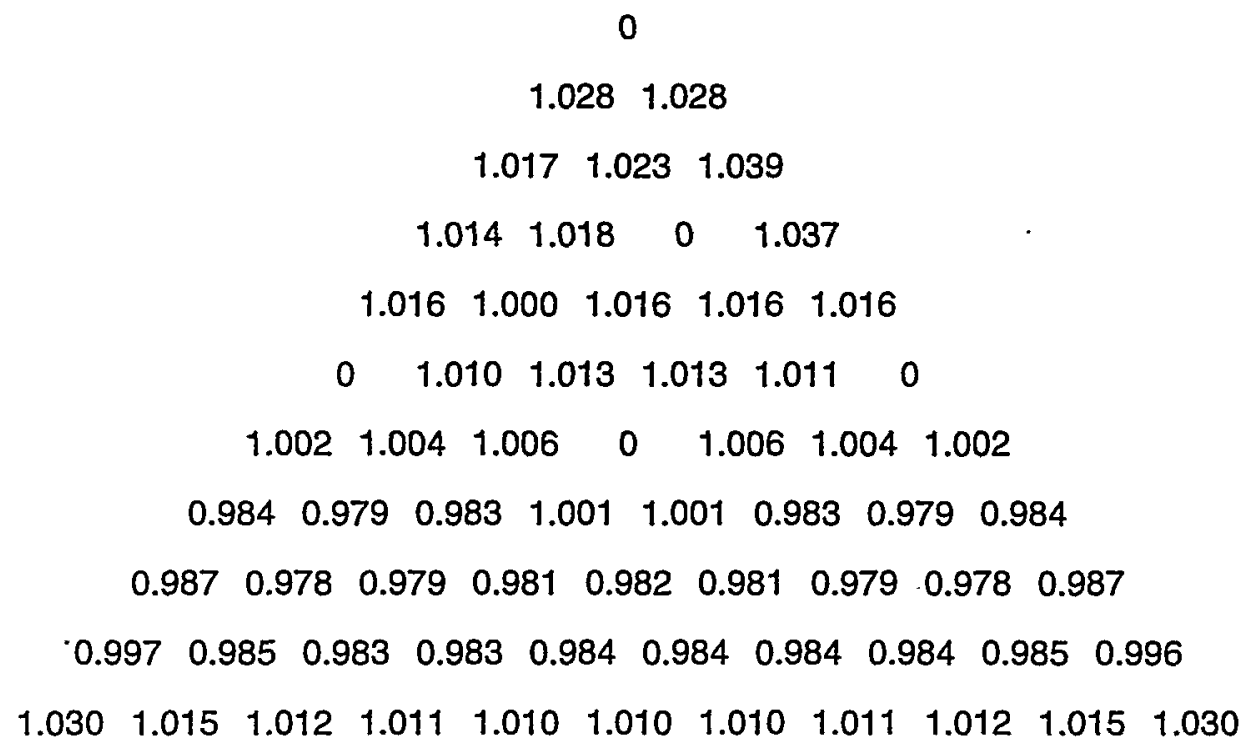

Fig. 4.20. Relative fission rate distribution for Variant 12, State 1 , at $40 \mathrm{MWd} / \mathrm{kg}$. 
0

1.0221 .022

$1.0121 .017 \quad 1.035$

$\begin{array}{llll}1.010 & 1.014 & 0 & 1.033\end{array}$

$\begin{array}{lllll}1.014 & 0.997 & 1.012 & 1.013 & 1.014\end{array}$

$\begin{array}{llllll}0 & 1.008 & 1.011 & 1.010 & 1.009 & 0\end{array}$

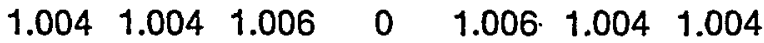

$\begin{array}{llllllll}0.989 & 0.983 & 0.986 & 1.002 & 1.002 & 0.986 & 0.983 & 0.989\end{array}$

$\begin{array}{lllllllll}0.991 & 0.982 & 0.983 & 0.985 & 0.985 & 0.985 & 0.983 & 0.982 & 0.991\end{array}$

$\begin{array}{llllllllll}0.996 & 0.986 & 0.985 & 0.985 & 0.985 & 0.985 & 0.985 & 0.985 & 0.986 & 0.995\end{array}$

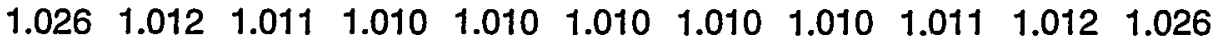

Fig. 4.21. Relative fission rate distribution for Variant 12, State 1 at $50 \mathrm{MWd} / \mathrm{kg}$.

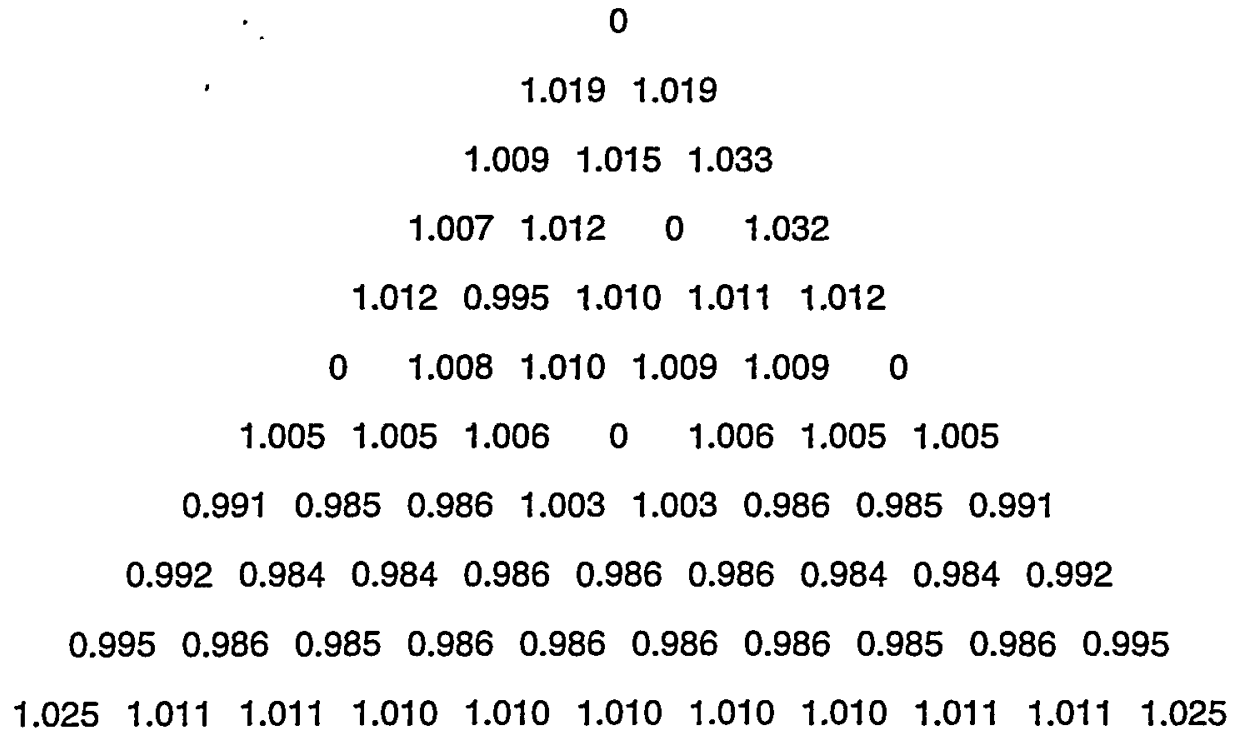

Fig. 4.22. Relative fission rate distribution for Variant 12, State 1 , at $60 \mathrm{MWd} / \mathrm{kg}$. 


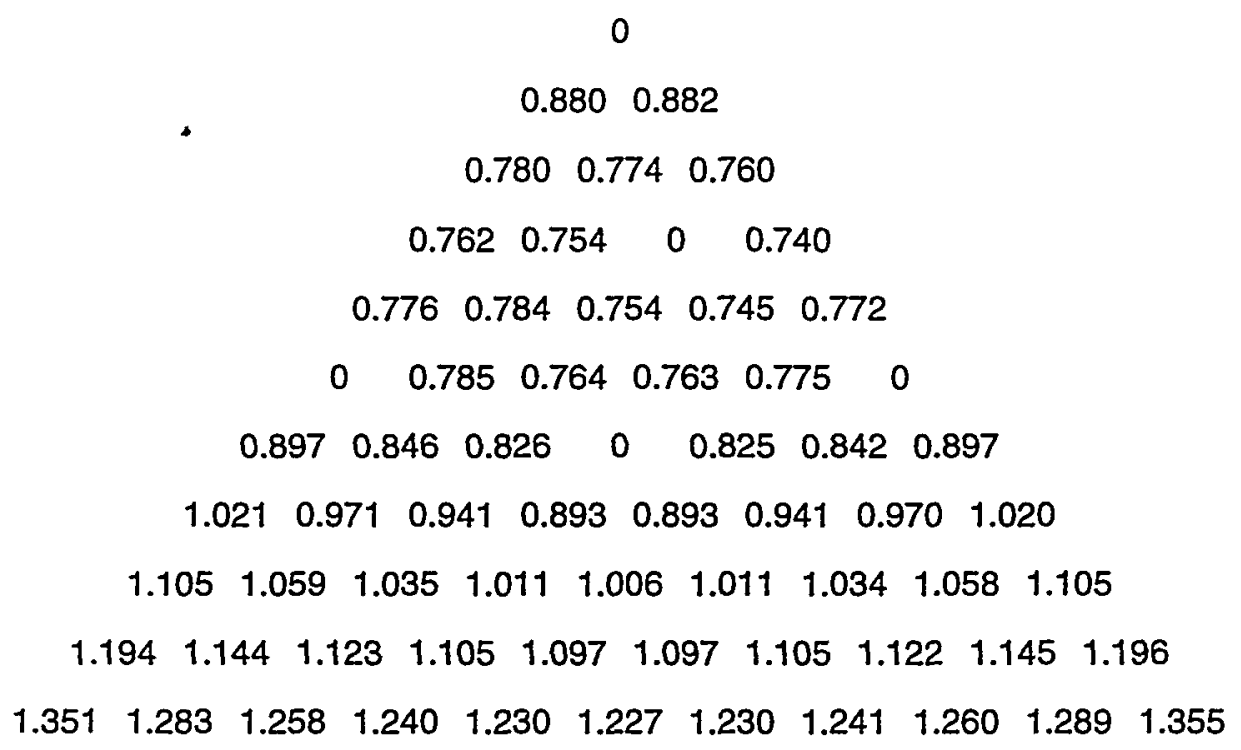

Fig. 4.23. Relative fission rate distribution for Variant 12, State 2 , at $0 \mathrm{MWd} / \mathrm{kg}$.

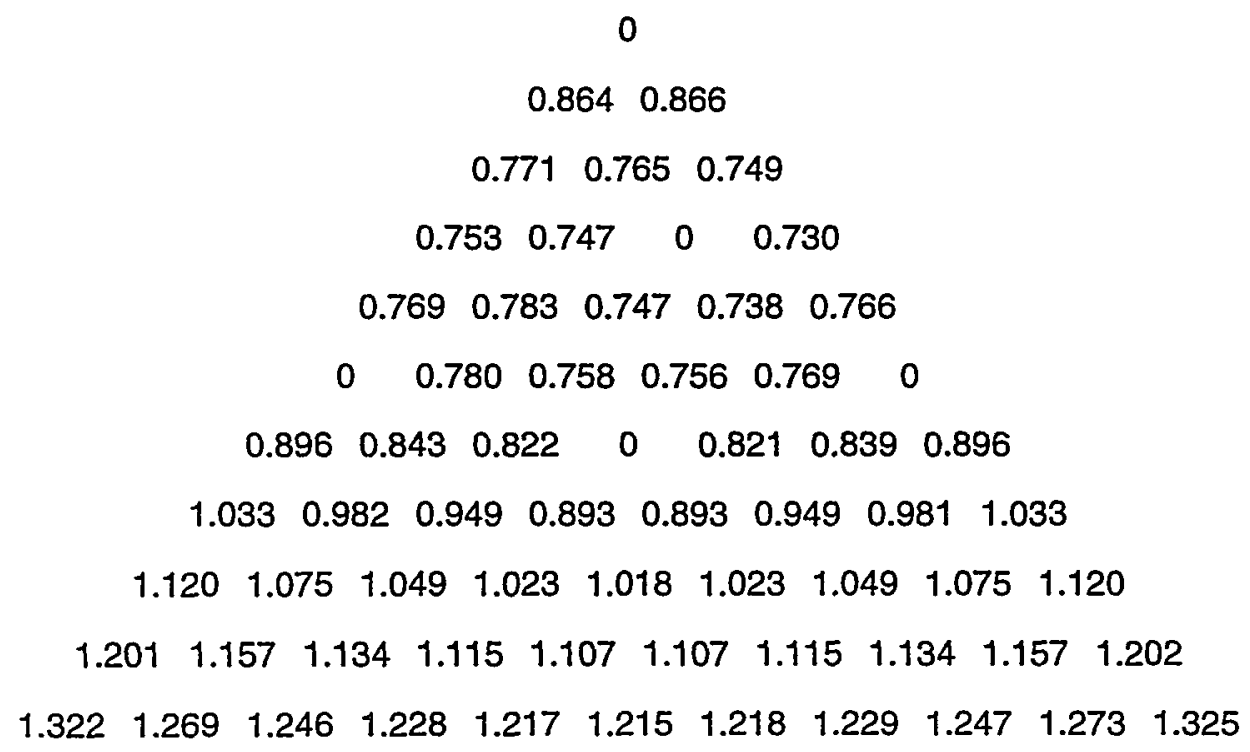

Fig. 4.24. Relative fission rate distribution for Variant 12, State 2, at $10 \mathrm{MWd} / \mathrm{kg}$. 


$$
\begin{aligned}
& 0 \\
& 0.845 \quad 0.847 \\
& \begin{array}{lll}
0.757 & 0.753 & 0.737
\end{array} \\
& \begin{array}{llll}
0.741 & 0.738 & 0 & 0.719
\end{array} \\
& \begin{array}{lllll}
0.761 & 0.778 & 0.738 & 0.728 & 0.757
\end{array} \\
& \begin{array}{llllll}
0 & 0.773 & 0.749 & 0.747 & 0.761 & 0
\end{array} \\
& \begin{array}{lllllll}
0.897 & 0.840 & 0.817 & 0 & 0.816 & 0.836 & 0.896
\end{array} \\
& \begin{array}{llllllll}
1.046 & 0.993 & 0.957 & 0.893 & 0.893 & 0.956 & 0.992 & 1.046
\end{array}
\end{aligned}
$$

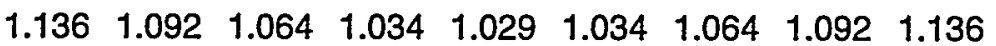

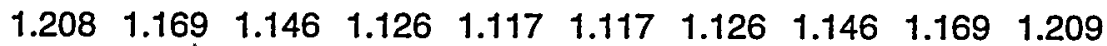

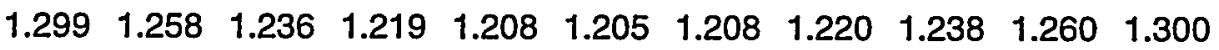

Fig. 4.25. Relative fission rate distribution for Variant 12, State 2, at $20 \mathrm{MWd} / \mathrm{kg}$.

$$
\begin{aligned}
& 0 \\
& 0.8260 .828 \\
& \begin{array}{lll}
0.743 & 0.740 & 0.727
\end{array} \\
& \begin{array}{llll}
0.728 & 0.728 & 0 & 0.710
\end{array} \\
& \begin{array}{lllll}
0.752 & 0.770 & 0.729 & 0.718 & 0.749
\end{array} \\
& \begin{array}{llllll}
0 & 0.767 & 0.741 & 0.739 & 0.754 & 0
\end{array} \\
& \begin{array}{lllllll}
0.898 & 0.838 & 0.814 & 0 & 0.813 & 0.834 & 0.897
\end{array} \\
& \begin{array}{llllllll}
1.058 & 1.003 & 0.963 & 0.894 & 0.894 & 0.962 & 1.001 & 1.058
\end{array}
\end{aligned}
$$

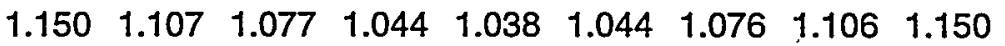

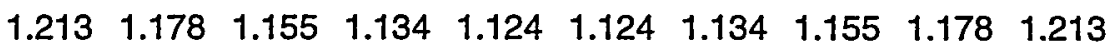

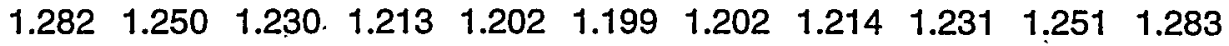

Fig. 4.26. Relative fission rate distribution for Variant 12, State 2, at $30 \mathrm{MWd} / \mathrm{kg}$. 


$$
\begin{aligned}
& 0 \\
& 0.809 \quad 0.812 \\
& \begin{array}{lll}
0.731 & 0.729 & 0.717
\end{array} \\
& \begin{array}{llll}
0.716 & 0.719 & 0 & 0.702
\end{array} \\
& \begin{array}{lllll}
0.745 & 0.761 & 0.721 & 0.710 & 0.742
\end{array} \\
& \begin{array}{llllll}
0 & 0.762 & 0.734 & 0.733 & 0.748 & 0
\end{array} \\
& \begin{array}{lllllll}
0.900 & 0.838 & 0.812 & 0 & 0.810 & 0.833 & 0.900
\end{array} \\
& \begin{array}{llllllll}
1.067 & 1.010 & 0.966 & 0.897 & 0.897 & 0.965 & 1.008 & 1.067
\end{array}
\end{aligned}
$$

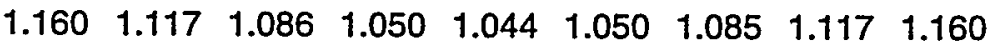

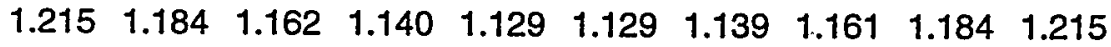

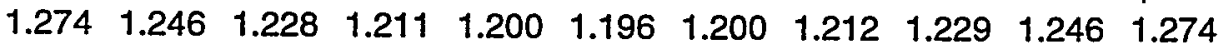

Fig. 4.27. Relative fission rate distribution for Variant 12, State 2, at $40 \mathrm{MWd} / \mathrm{kg}$.

$$
\begin{aligned}
& 0 \\
& 0.798 \quad 0.800 \\
& \begin{array}{lll}
0.722 & 0.720 & 0.711
\end{array} \\
& \begin{array}{llll}
0.708 & 0.712 & 0 & 0.697
\end{array} \\
& \begin{array}{lllll}
0.740 & 0.754 & 0.715 & 0.705 & 0.737
\end{array} \\
& \begin{array}{llllll}
0 & 0.759 & 0.729 & 0.728 & 0.745 & 0
\end{array} \\
& \begin{array}{lllllll}
0.902 & 0.838 & 0.811 & 0 & 0.809 & 0.833 & 0.902
\end{array}
\end{aligned}
$$

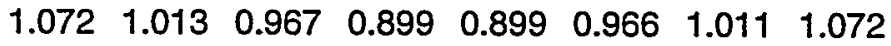

$$
\begin{aligned}
& \begin{array}{lllllllll}
1.166 & 1.123 & 1.090 & 1.053 & 1.047 & 1.053 & 1.090 & 1.122 & 1.166
\end{array}
\end{aligned}
$$

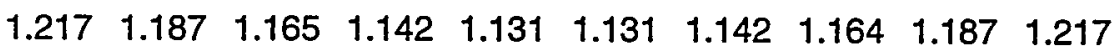

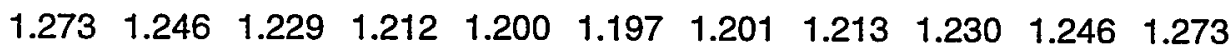

Fig. 4.28. Relative fission rate distribution for Variant 12, State 2, at $50 \mathrm{MWd} / \mathrm{kg}$. 


$$
\begin{aligned}
& 0 \\
& 0.788 \quad 0.790 \\
& 0.714 \quad 0.714 \quad 0.705 \\
& \begin{array}{llll}
0.702 & 0.706 & 0 & 0.692
\end{array} \\
& \begin{array}{lllll}
0.736 & 0.748 & 0.711 & 0.700 & 0.733
\end{array} \\
& \begin{array}{llllll}
0 & 0.756 & 0.726 & 0.725 & 0.742 & 0
\end{array} \\
& \begin{array}{lllllll}
0.904 & 0.839 & 0.810 & 0 & 0.809 & 0.834 & 0.903
\end{array} \\
& \begin{array}{llllllll}
1.075 & 1.014 & 0.967 & 0.901 & 0.900 & 0.966 & 1.012 & 1.074
\end{array}
\end{aligned}
$$

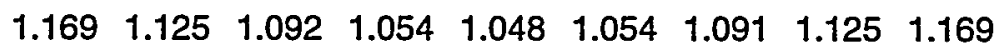

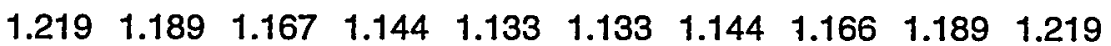

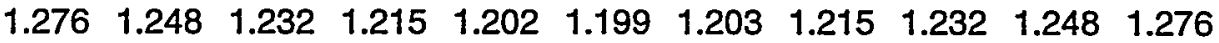

Fig. 4.29. Relative fission rate distribution for Variant 12 , State 2 , at $60 \mathrm{MWd} / \mathrm{kg}$. 


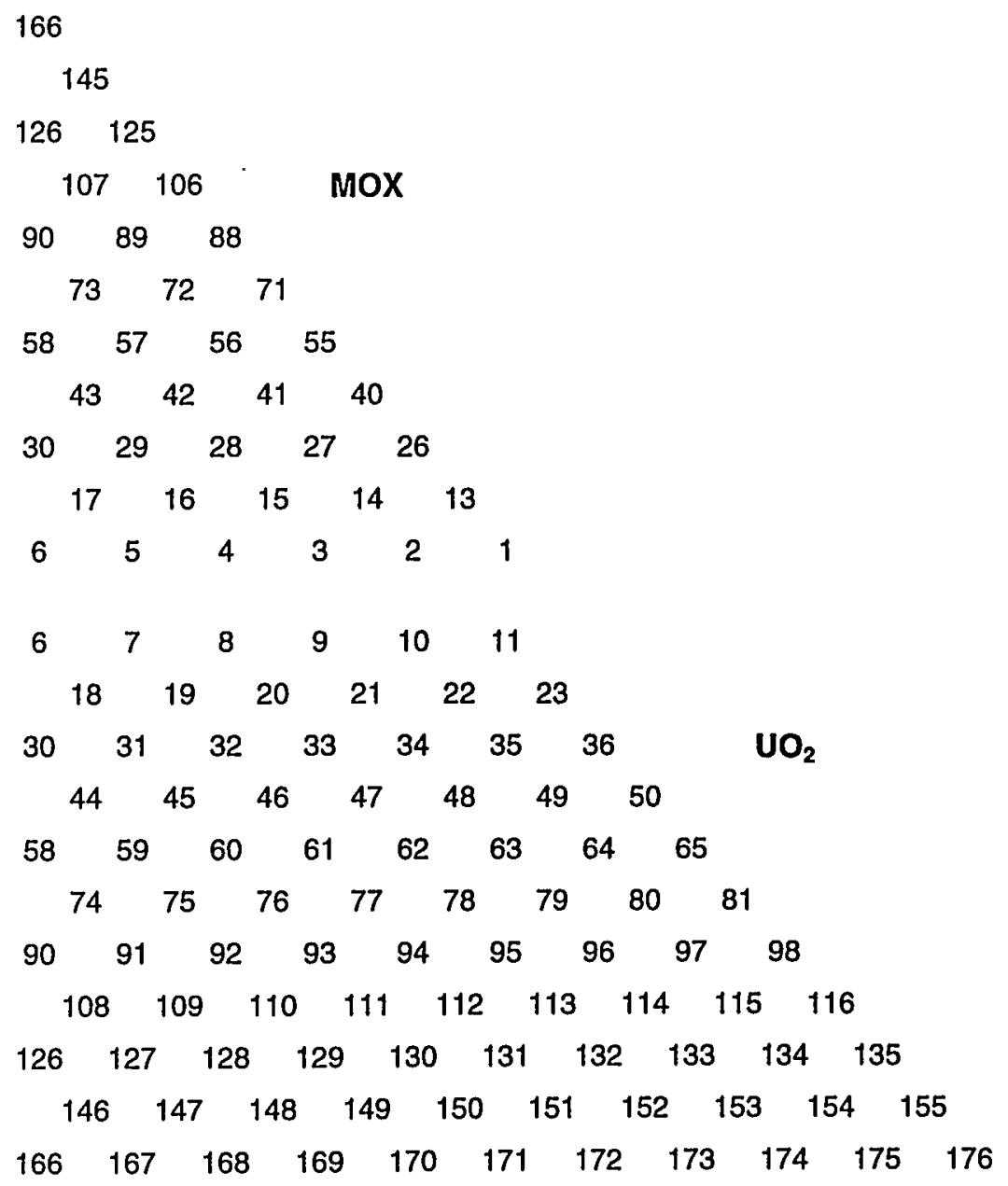

Fig. 4.30. Pin numbering for Variants 13 and 14.
0.856

0.836

0.8250 .838

0.8310 .842

$\begin{array}{lll}0.832 & 0.834 & 0.846\end{array}$

$\begin{array}{llll}0.841 & 0.840 & 0.853\end{array}$

$\begin{array}{llll}0.847 & 0.849 & 0.851 & 0.868\end{array}$

$\begin{array}{lllll}0.868 & 0.868 & 0.873 & 0.897\end{array}$

$\begin{array}{lllll}0.900 & 0.904 & 0.909 & 0.919 & 0.959\end{array}$

$\begin{array}{lllll}0.983 & 0.991 & 1.001 & 1.021 & 1.092\end{array}$

$\begin{array}{llllll}1.185 & 1.195 & 1.206 & 1.222 & 1.263 & 1.381\end{array}$

$\begin{array}{llllll}0.947 & 0.947 & 0.953 & 0.965 & 0.992 & 1.047\end{array}$

$\begin{array}{llllll}0.961 & 0.963 & 0.971 & 0.986 & 1.018 & 1.084\end{array}$

$\begin{array}{lllllll}0.972 & 0.973 & 0.977 & 0.986 & 1.003 & 1.038 & 1.100\end{array}$

$\begin{array}{lllllll}0.980 & 0.981 & 0.986 & 0.996 & 1.014 & 1.048 & 1.109\end{array}$

$\begin{array}{llllllll}0.982 & 0.983 & 0.986 & 0.992 & 1.002 & 1.020 & 1.053 & 1.113\end{array}$

$\begin{array}{llllllll}0.985 & 0.986 & 0.989 & 0.995 & 1.005 & 1.023 & 1.056 & 1.116\end{array}$

$\begin{array}{lllllllll}0.985 & 0.985 & 0.987 & 0.991 & 0.997 & 1.007 & 1.026 & 1.059 & 1.119\end{array}$

$\begin{array}{lllllllll}0.986 & 0.986 & 0.988 & 0.992 & 0.998 & 1.009 & 1.028 & 1.062 & 1.123\end{array}$

$\begin{array}{llllllllll}0.985 & 0.985 & 0.987 & 0.989 & 0.993 & 1.000 & 1.012 & 1.032 & 1.067 & 1.130\end{array}$

$\begin{array}{llllllllll}0.986 & 0.986 & 0.987 & 0.990 & 0.995 & 1.002 & 1.015 & 1.037 & 1.075 & 1.141\end{array}$

$\begin{array}{lllllllllll}0.993 & 0.991 & 0.992 & 0.994 & 0.997 & 1.003 & 1.013 & 1.029 & 1.055 & 1.097 & 1.171\end{array}$

Fig. 4.31. Relative fission rate distribution for Variant 13, State 1 , at $0 \mathrm{MWd} / \mathrm{kg}$. 


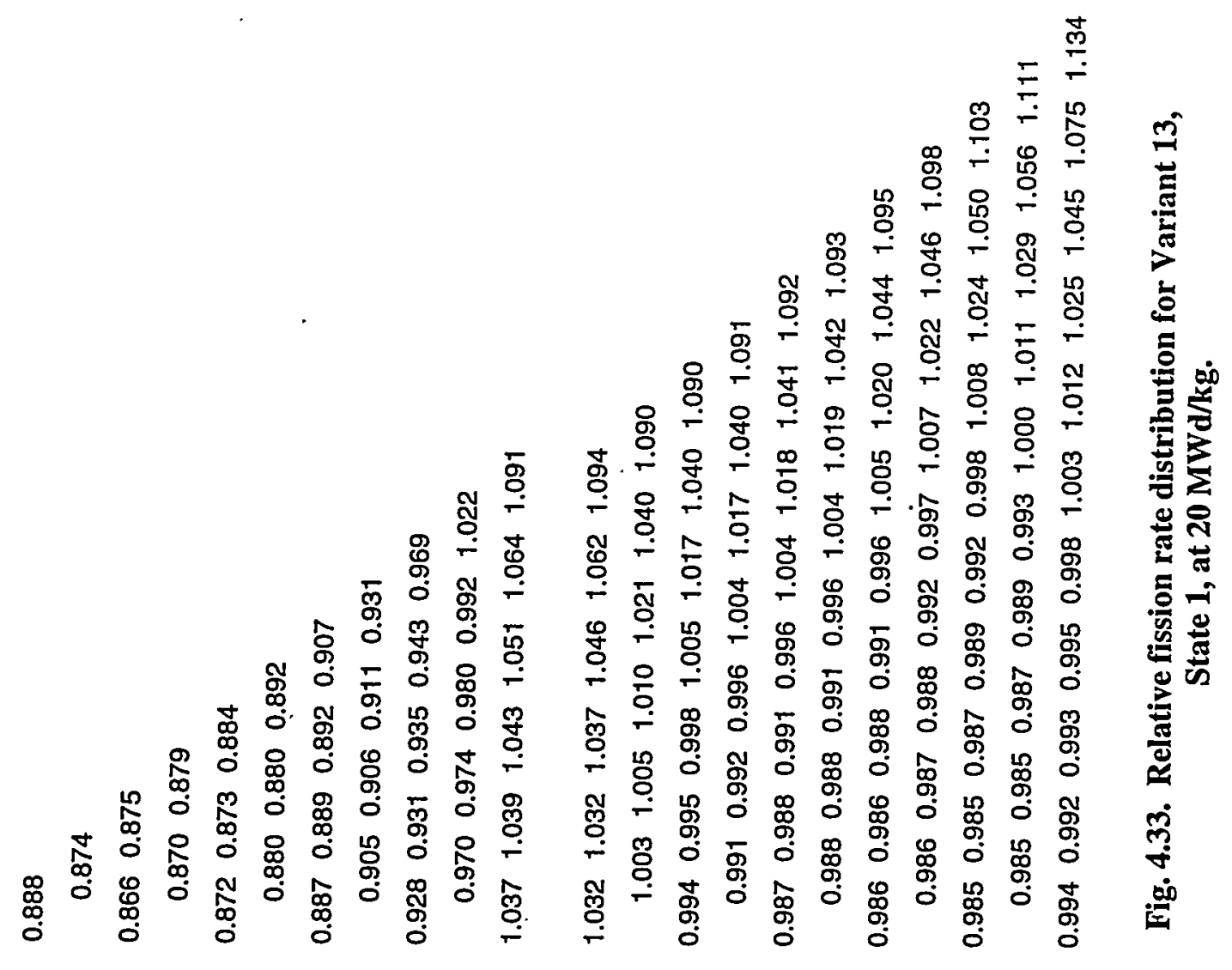

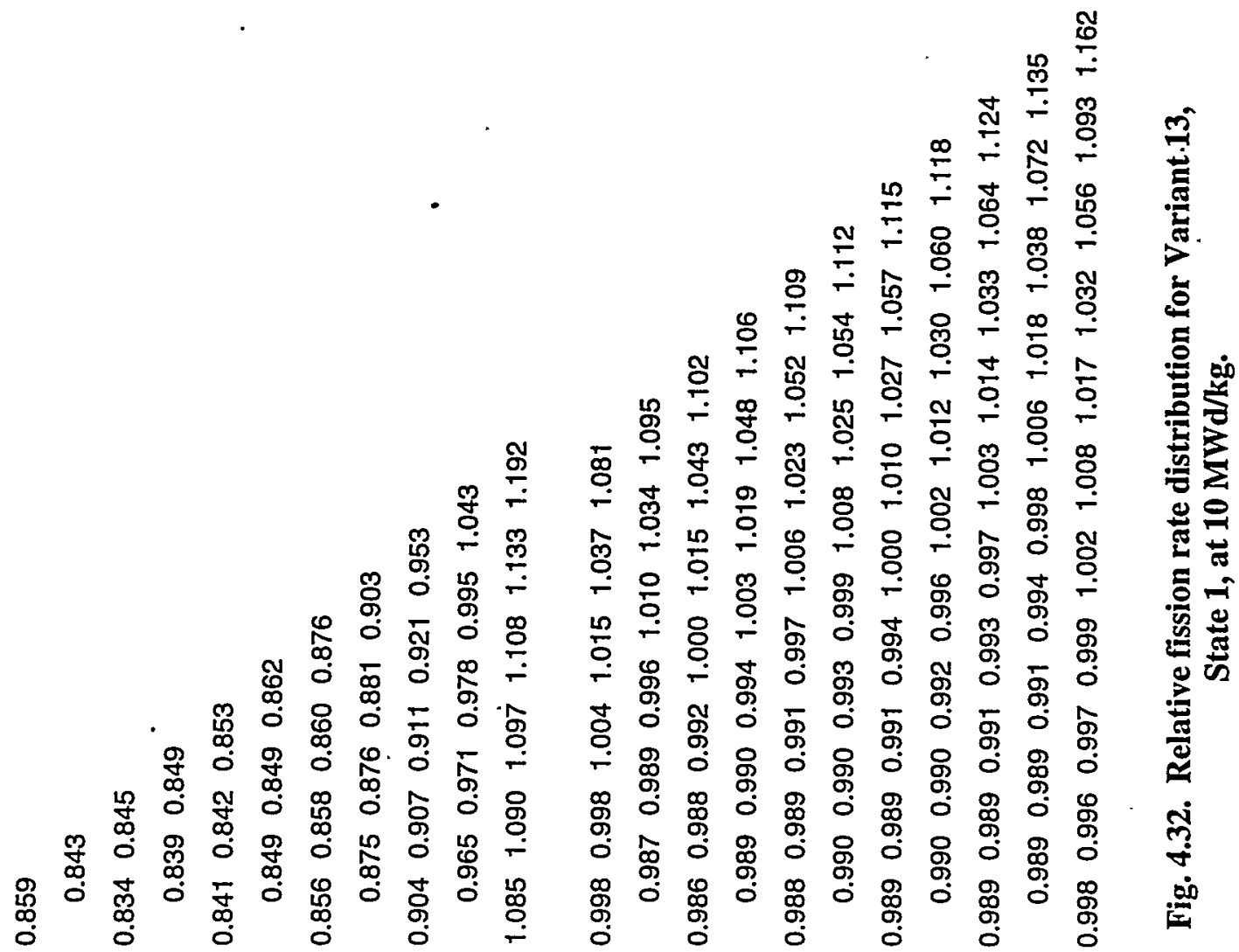


0.915

0.902

0.8940 .903

$0.898 \quad 0.907$

$\begin{array}{lll}0.901 & 0.902 & 0.912\end{array}$

$\begin{array}{lll}0.908 & 0.909 & 0.920\end{array}$

$\begin{array}{llll}0.915 & 0.916 & 0.919 & 0.933\end{array}$

$\begin{array}{llll}0.930 & 0.931 & 0.936 & 0.952\end{array}$

$\begin{array}{lllll}0.947 & 0.949 & 0.953 & 0.959 & 0.978\end{array}$

$\begin{array}{lllll}0.972 & 0.974 & 0.978 & 0.986 & 1.002\end{array}$

$\begin{array}{llllll}1.009 & 1.009 & 1.011 & 1.016 & 1.021 & 1.035\end{array}$

$\begin{array}{llllll}1.051 & 1.052 & 1.055 & 1.062 & 1.073 & 1.095\end{array}$ $\begin{array}{llllll}1.013 & 1.014 & 1.018 & 1.027 & 1.039 & 1.080\end{array}$ $\begin{array}{lllllll}0.993 & 0.994 & 0.998 & 1.004 & 1.015 & 1.032 & 1.075\end{array}$

$\begin{array}{llllllll}0.988 & 0.989 & 0.991 & 0.995 & 1.002 & 1.013 & 1.031 & 1.074\end{array}$ $\begin{array}{llllllll}0.987 & 0.988 & 0.990 & 0.994 & 1.002 & 1.013 & 1.031 & 1.075\end{array}$

$\begin{array}{lllllllll}0.985 & 0.986 & 0.987 & 0.990 & 0.994 & 1.002 & 1.014 & 1.032 & 1.076\end{array}$ $\begin{array}{lllllllll}0.985 & 0.985 & 0.987 & 0.990 & 0.995 & 1.003 & 1.015 & 1.034 & 1.079\end{array}$

$\begin{array}{llllllllll}0.983 & 0.984 & 0.985 & 0.987 & 0.990 & 0.996 & 1.004 & 1.017 & 1.037 & 1.082\end{array}$ $\begin{array}{llllllllll}0.984 & 0.984 & 0.985 & 0.987 & 0.991 & 0.997 & 1.007 & 1.021 & 1.042 & 1.088\end{array}$

$\begin{array}{lllllllllll}0.992 & 0.991 & 0.991 & 0.993 & 0.996 & 1.001 & 1.008 & 1.019 & 1.036 & 1.058 & 1.108\end{array}$

Fig. 4.34. Relative fission rate distribution for Variant 13, State 1 , at $30 \mathrm{MWd} / \mathrm{kg}$.
0.941

0.929

$0.922 \quad 0.930$

0.9250 .934

$\begin{array}{llll}0.928 & 0.929 & 0.938\end{array}$

$\begin{array}{lll}0.934 & 0.935 & 0.946\end{array}$

$\begin{array}{lllll}0.940 & 0.942 & 0.944 & 0.956\end{array}$

$\begin{array}{llll}0.952 & 0.953 & 0.957 & 0.970\end{array}$

$\begin{array}{lllll}0.963 & 0.964 & 0.967 & 0.971 & 0.985\end{array}$

$\begin{array}{lllll}0.975 & 0.976 & 0.978 & 0.982 & 0.992\end{array}$

$\begin{array}{llllll}0.998 & 0.998 & 1.000 & 1.002 & 1.004 & 1.014\end{array}$

$\begin{array}{llllll}1.059 & 1.060 & 1.063 & 1.067 & 1.072 & 1.087\end{array}$

$\begin{array}{llllll}1.017 & 1.018 & 1.021 & 1.026 & 1.033 & 1.068\end{array}$

$\begin{array}{lllllll}1.002 & 1.003 & 1.005 & 1.009 & 1.016 & 1.026 & 1.062\end{array}$

$\begin{array}{lllllll}0.995 & 0.996 & 0.998 & 1.003 & 1.011 & 1.022 & 1.059\end{array}$

$\begin{array}{llllllllll}0.989 & 0.990 & 0.992 & 0.995 & 1.000 & 1.009 & 1.021 & 1.058\end{array}$

$\begin{array}{llllllll}0.987 & 0.988 & 0.990 & 0.993 & 0.999 & 1.008 & 1.020 & 1.058\end{array}$

$\begin{array}{lllllllll}0.984 & 0.985 & 0.986 & 0.989 & 0.993 & 0.999 & 1.008 & 1.021 & 1.059\end{array}$

$\begin{array}{lllllllll}0.984 & 0.985 & 0.986 & 0.988 & 0.993 & 0.999 & 1.009 & 1.022 & 1.061\end{array}$

$\begin{array}{llllllllll}0.982 & 0.983 & 0.984 & 0.986 & 0.989 & 0.993 & 1.000 & 1.010 & 1.024 & 1.063\end{array}$

$\begin{array}{llllllllll}0.983 & 0.983 & 0.984 & 0.986 & 0.989 & 0.994 & 1.002 & 1.013 & 1.028 & 1.067\end{array}$

$\begin{array}{lllllllllll}0.991 & 0.990 & 0.991 & 0.992 & 0.994 & 0.998 & 1.005 & 1.013 & 1.026 & 1.041 & 1.083\end{array}$

Fig. 4.35. Relative fission rate distribution for Variant 13, State 1 , at $40 \mathrm{MWd} / \mathrm{kg}$. 
0.967

0.955

$0.948 \quad 0.957$

0.9510 .960

$0.953 \quad 0.9540 .963$

$0.958 \quad 0.9590 .969$

$\begin{array}{llll}0.963 & 0.964 & 0.966 & 0.977\end{array}$

$\begin{array}{llll}0.971 & 0.972 & 0.974 & 0.985\end{array}$

$\begin{array}{lllll}0.977 & 0.978 & 0.979 & 0.982 & 0.992\end{array}$

$\begin{array}{lllll}0.982 & 0.982 & 0.983 & 0.985 & 0.992\end{array}$

. 1.0021 .0021 .0021 .0041 .0051 .014

$\begin{array}{llllll}1.060 & 1.061 & 1.062 & 1.064 & 1.066 & 1.077\end{array}$

$\begin{array}{llllll}1.017 & 1.017 & 1.019 & 1.022 & 1.026 & 1.056\end{array}$ $\begin{array}{lllllll}0.995 & 0.996 & 0.998 & 1.001 & 1.006 & 1.013 & 1.047\end{array}$

$\begin{array}{lllllllll}0.988 & 0.990 & 0.991 & 0.994 & 0.997 & 1.003 & 1.011 & 1.045\end{array}$

$\begin{array}{llllllll}0.987 & 0.987 & 0.989 & 0.991 & 0.996 & 1.002 & 1.010 & 1.044\end{array}$

$\begin{array}{llllllllll}0.983 & 0.984 & 0.985 & 0.987 & 0.990 & 0.995 & 1.001 & 1.010 & 1.045\end{array}$

$\begin{array}{lllllllll}0.983 & 0.984 & 0.985 & 0.987 & 0.990 & 0.995 & 1.002 & 1.011 & 1.045\end{array}$

$\begin{array}{lllllllllllll}0.981 & 0.982 & 0.983 & 0.984 & 0.987 & 0.990 & 0.995 & 1.003 & 1.012 & 1.047\end{array}$

$\begin{array}{llllllllll}0.982 & 0.982 & 0.983 & 0.984 & 0.987 & 0.991 & 0.996 & 1.004 & 1.014 & 1.049\end{array}$

$\begin{array}{lllllllllll}0.989 & 0.989 & 0.989 & 0.991 & 0.993 & 0.996 & 1.000 & 1.007 & 1.016 & 1.025 & 1.064\end{array}$

Fig. 4.36. Relative fission rate distribution for Variant 13, State 1 , at $50 \mathrm{MWd} / \mathrm{kg}$.
0.990

0.978

0.9700 .980

$0.973 \quad 0.982$

$\begin{array}{lll}0.975 & 0.976 \quad 0.985\end{array}$

0.9790 .9790 .989

$\begin{array}{llll}0.981 & 0.983 & 0.984 & 0.994\end{array}$

$\begin{array}{llll}0.987 & 0.987 & 0.988 & 0.998\end{array}$

$\begin{array}{lllll}0.989 & 0.990 & 0.991 & 0.992 & 1.000\end{array}$

$\begin{array}{lllll}0.991 & 0.991 & 0.991 & 0.992 & 0.999\end{array}$

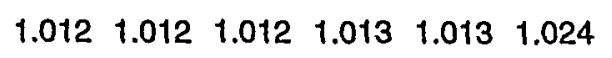

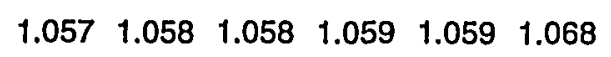

$\begin{array}{llllll}1.014 & 1.014 & 1.015 & 1.017 & 1.018 & 1.047\end{array}$

$\begin{array}{lllllll}1.000 & 1.002 & 1.002 & 1.004 & 1.007 & 1.010 & 1.041\end{array}$

$\begin{array}{lllllll}0.994 & 0.994 & 0.995 & 0.997 & 1.001 & 1.005 & 1.038\end{array}$

$\begin{array}{llllllll}0.987 & 0.988 & 0.989 & 0.991 & 0.994 & 0.998 & 1.003 & 1.036\end{array}$

$\begin{array}{llllllll}0.985 & 0.985 & 0.987 & 0.989 & 0.991 & 0.996 & 1.002 & 1.035\end{array}$

$\begin{array}{lllllllll}0.982 & 0.983 & 0.984 & 0.985 & 0.987 & 0.990 & 0.995 & 1.001 & 1.035\end{array}$

$\begin{array}{lllllllll}0.982 & 0.982 & 0.983 & 0.984 & 0.986 & 0.990 & 0.995 & 1.001 & 1.035\end{array}$

$\begin{array}{llllllllll}0.980 & 0.981 & 0.981 & 0.982 & 0.984 & 0.986 & 0.990 & 0.995 & 1.002 & 1.036\end{array}$

$\begin{array}{llllllllll}0.980 & 0.980 & 0.981 & 0.982 & 0.984 & 0.987 & 0.991 & 0.996 & 1.003 & 1.037\end{array}$

$\begin{array}{lllllllllll}0.988 & 0.988 & 0.988 & 0.989 & 0.990 & 0.993 & 0.996 & 1.001 & 1.007 & 1.014 & 1.052\end{array}$

Fig. 4.37. Relative fission rate distribution for Variant 13, State 1 , at $60 \mathrm{MWd} / \mathrm{kg}$. 
0.830

0.811

0.8010 .813

$0.807 \quad 0.818$

$\begin{array}{lll}0.809 & 0.810 & 0.823\end{array}$

$\begin{array}{lll}0.818 & 0.818 & 0.831\end{array}$

$\begin{array}{lllll}0.826 & 0.827 & 0.830 & 0.847\end{array}$

$\begin{array}{llll}0.847 & 0.848 & 0.853 & 0.879\end{array}$

$\begin{array}{lllll}0.882 & 0.885 & 0.891 & 0.902 & 0.943\end{array}$

$\begin{array}{lllll}0.966 & 0.975 & 0.985 & 1.006 & 1.078\end{array}$

$\begin{array}{llllll}1.167 & 1.178 & 1.189 & 1.207 & 1.248 & 1.367\end{array}$

$\begin{array}{llllll}0.941 & 0.941 & 0.947 & 0.961 & 0.989 & 1.044\end{array}$

$\begin{array}{llllll}0.959 & 0.961 & 0.969 & 0.986 & 1.018 & 1.085\end{array}$

$\begin{array}{llllllll}0.973 & 0.974 & 0.979 & 0.988 & 1.006 & 1.041 & 1.104\end{array}$

$\begin{array}{lllllll}0.983 & 0.984 & 0.990 & 1.000 & 1.019 & 1.054 & 1.114\end{array}$

$\begin{array}{llllllll}0.987 & 0.988 & 0.991 & 0.997 & 1.008 & 1.027 & 1.061 & 1.121\end{array}$

$\begin{array}{llllllll}0.991 & 0.992 & 0.995 & 1.002 & 1.013 & 1.032 & 1.066 & 1.125\end{array}$

$\begin{array}{lllllllll}0.992 & 0.992 & 0.994 & 0.998 & 1.005 & 1.016 & 1.036 & 1.069 & 1.129\end{array}$

$\begin{array}{lllllllll}0.994 & 0.994 & 0.996 & 1.001 & 1.008 & 1.019 & 1.039 & 1.073 & 1.134\end{array}$

$\begin{array}{llllllllll}0.993 & 0.994 & 0.995 & 0.998 & 1.002 & 1.010 & 1.022 & 1.043 & 1.079 & 1.141\end{array}$

$\begin{array}{llllllllll}0.994 & 0.994 & 0.996 & 0.999 & 1.004 & 1.013 & 1.027 & 1.049 & 1.088 & 1.153\end{array}$

$\begin{array}{lllllllllll}1.002 & 0.999 & 1.001 & 1.003 & 1.007 & 1.013 & 1.024 & 1.040 & 1.067 & 1.110 & 1.183\end{array}$

Fig. 4.38. Relative fission rate distribution for Variant 13, . State 4 , at $0 \mathrm{MWd} / \mathrm{kg}$.
0.850

0.834

0.8250 .836

$0.831 \quad 0.840$

$\begin{array}{lll}0.833 & 0.834 & 0.845\end{array}$

$\begin{array}{lll}0.841 & 0.841 & 0.854\end{array}$

$\begin{array}{llll}0.849 & 0.851 & 0.854 & 0.870\end{array}$

$\begin{array}{llll}0.870 & 0.871 & 0.876 & 0.899\end{array}$

$\begin{array}{lllll}0.900 & 0.902 & 0.908 & 0.918 & 0.951\end{array}$

$\begin{array}{lllll}0.962 & 0.968 & 0.976 & 0.993 & 1.042\end{array}$

$\begin{array}{lllllll}1.080 & 1.086 & 1.093 & 1.105 & 1.130 & 1.189\end{array}$

$\begin{array}{llllll}0.995 & 0.995 & 1.001 & 1.012 & 1.035 & 1.079\end{array}$

$\begin{array}{lllllll}0.986 & 0.988 & 0.995 & 1.009 & 1.034 & 1.094\end{array}$

$\begin{array}{lllllll}0.987 & 0.988 & 0.992 & 1.000 & 1.016 & 1.044 & 1.102\end{array}$

$\begin{array}{lllllll}0.989 & 0.991 & 0.995 & 1.005 & 1.021 & 1.050 & 1.107\end{array}$

$\begin{array}{llllllll}0.989 & 0.990 & 0.993 & 0.998 & 1.008 & 1.025 & 1.054 & 1.110\end{array}$

$\begin{array}{llllllll}0.991 & 0.992 & 0.995 & 1.001 & 1.011 & 1.028 & 1.057 & 1.113\end{array}$

$\begin{array}{lllllllll}0.990 & 0.991 & 0.993 & 0.997 & 1.003 & 1.013 & 1.030 & 1.060 & 1.117\end{array}$

$\begin{array}{lllllllll}0.992 & 0.992 & 0.994 & 0.998 & 1.005 & 1.015 & 1.033 & 1.063 & 1.121\end{array}$

$\begin{array}{llllllllll}0.991 & 0.992 & 0.993 & 0.995 & 1.000 & 1.006 & 1.018 & 1.037 & 1.068 & 1.127\end{array}$

$\begin{array}{llllllllll}0.992 & 0.992 & 0.993 & 0.996 & 1.001 & 1.009 & 1.022 & 1.042 & 1.076 & 1.137\end{array}$

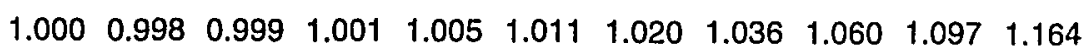

Fig. 4.39. Relative fission rate distribution for Variant 13, State 4 , at $10 \mathrm{MWd} / \mathrm{kg}$. 


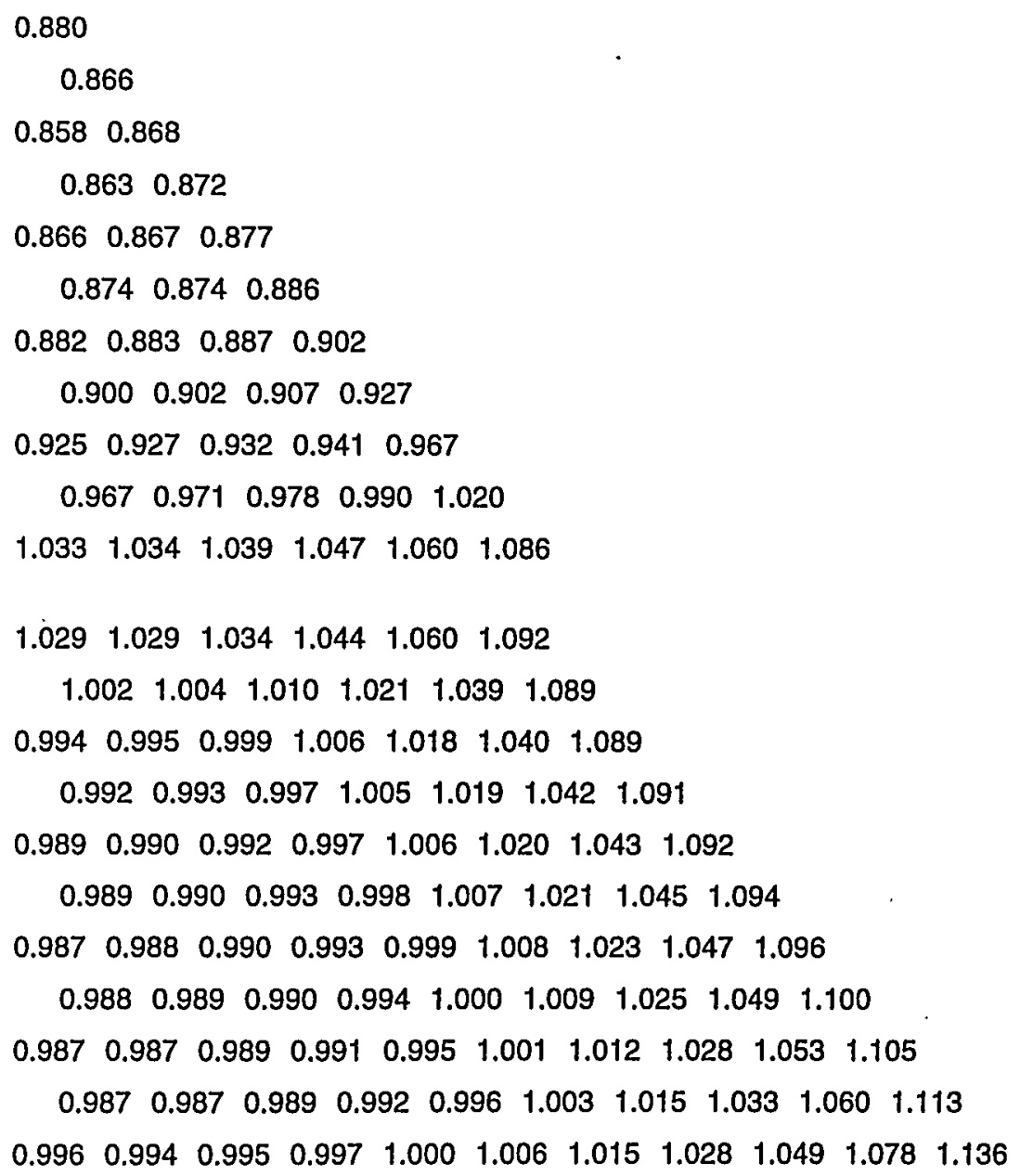

Fig. 4.40. Relative fission rate distribution for Variant 13, State 4, at $20 \mathrm{MWd} / \mathrm{kg}$.
0.908

0.895

$0.888 \quad 0.897$

0.8930 .901

$\begin{array}{lll}0.896 & 0.897 & 0.907\end{array}$ $\begin{array}{lll}0.903 & 0.904 \quad 0.916\end{array}$

$\begin{array}{llll}0.911 & 0.913 & 0.916 & 0.930\end{array}$ $\begin{array}{llll}0.927 & 0.928 & 0.933 & 0.950\end{array}$

$\begin{array}{llllll}0.945 & 0.947 & 0.951 & 0.958 & 0.976\end{array}$ $\begin{array}{lllll}0.970 & 0.972 & 0.976 & 0.984 & 1.000\end{array}$ $\begin{array}{llllll}1.004 & 1.005 & 1.007 & 1.012 & 1.018 & 1.030\end{array}$

$\begin{array}{llllll}1.048 & 1.049 & 1.053 & 1.060 & 1.070 & 1.092\end{array}$ $\begin{array}{llllll}1.012 & 1.014 & 1.018 & 1.026 & 1.039 & 1.078\end{array}$ $\begin{array}{lllllll}0.999 & 1.001 & 1.004 & 1.009 & 1.019 & 1.034 & 1.075\end{array}$ $\begin{array}{lllllll}0.994 & 0.995 & 0.999 & 1.005 & 1.016 & 1.033 & 1.074\end{array}$ $\begin{array}{llllllll}0.989 & 0.990 & 0.993 & 0.997 & 1.004 & 1.015 & 1.032 & 1.074\end{array}$ $\begin{array}{llllllll}0.989 & 0.989 & 0.992 & 0.996 & 1.004 & 1.016 & 1.033 & 1.075\end{array}$ $\begin{array}{lllllllll}0.986 & 0.987 & 0.989 & 0.992 & 0.996 & 1.004 & 1.016 & 1.034 & 1.077\end{array}$ $\begin{array}{lllllllll}0.987 & 0.987 & 0.989 & 0.992 & 0.997 & 1.005 & 1.018 & 1.036 & 1.079\end{array}$ $\begin{array}{llllllllll}0.985 & 0.986 & 0.987 & 0.989 & 0.992 & 0.998 & 1.007 & 1.020 & 1.039 & 1.083\end{array}$ $\begin{array}{llllllllll}0.985 & 0.985 & 0.987 & 0.989 & 0.993 & 1.000 & 1.010 & 1.024 & 1.044 & 1.089\end{array}$ $\begin{array}{lllllllllll}0.993 & 0.992 & 0.993 & 0.995 & 0.998 & 1.003 & 1.011 & 1.022 & 1.039 & 1.060 & 1.107\end{array}$

Fig. 4.41. Relative fission rate distribution for Variant 13, State 4, at $30 \mathrm{MWd} / \mathrm{kg}$. 
0.936

0.924

$0.918 \quad 0.926$

0.9220 .930

$\begin{array}{lll}0.924 & 0.925 & 0.935\end{array}$

$\begin{array}{lll}0.931 & 0.932 \quad 0.943\end{array}$

$\begin{array}{llll}0.938 & 0.939 & 0.942 & 0.954\end{array}$

$\begin{array}{llll}0.950 & 0.951 & 0.955 & 0.969\end{array}$

$\begin{array}{lllll}0.962 & 0.963 & 0.966 & 0.970 & 0.983\end{array}$

$\begin{array}{lllll}0.973 & 0.974 & 0.977 & 0.980 & 0.989\end{array}$

$\begin{array}{llllll}0.995 & 0.994 & 0.996 & 0.998 & 1.000 & 1.009\end{array}$

$\begin{array}{llllll}1.057 & 1.058 & 1.060 & 1.065 & 1.070 & 1.084\end{array}$

$\begin{array}{llllll}1.017 & 1.018 & 1.021 & 1.026 & 1.033 & 1.066\end{array}$

$\vec{A}$ $\begin{array}{lllllll}1.002 & 1.004 & 1.006 & 1.010 & 1.017 & 1.026 & 1.060\end{array}$

$\begin{array}{lllllll}0.996 & 0.997 & 1.000 & 1.004 & 1.012 & 1.023 & 1.058\end{array}$

$\begin{array}{llllllll}0.990 & 0.991 & 0.993 & 0.996 & 1.002 & 1.010 & 1.022 & 1.057\end{array}$

$\begin{array}{llllllll}0.988 & 0.989 & 0.991 & 0.995 & 1.001 & 1.010 & 1.021 & 1.058\end{array}$

$\begin{array}{lllllllll}0.986 & 0.987 & 0.988 & 0.990 & 0.994 & 1.001 & 1.010 & 1.022 & 1.058\end{array}$

$\begin{array}{lllllllll}0.986 & 0.986 & 0.988 & 0.990 & 0.995 & 1.001 & 1.011 & 1.023 & 1.060\end{array}$

$\begin{array}{llllllllll}0.984 & 0.985 & 0.986 & 0.988 & 0.991 & 0.995 & 1.002 & 1.012 & 1.025 & 1.063\end{array}$

$\begin{array}{llllllllll}0.984 & 0.984 & 0.986 & 0.988 & 0.991 & 0.997 & 1.004 & 1.015 & 1.029 & 1.066\end{array}$

$\begin{array}{lllllllllll}0.992 & 0.991 & 0.992 & 0.994 & 0.996 & 1.001 & 1.007 & 1.016 & 1.028 & 1.042 & 1.082\end{array}$

Fig. 4.42. Relative fission rate distribution for Variant 13, State 4 , at $40 \mathrm{MWd} / \mathrm{kg}$.
0.964

0.952

$0.946 \quad 0.954$

0.9490 .957

$\begin{array}{lll}0.951 & 0.952 & 0.962\end{array}$

$\begin{array}{lll}0.957 & 0.958 & 0.968\end{array}$

$\begin{array}{llll}0.962 & 0.963 & 0.965 & 0.976\end{array}$

$\begin{array}{llll}0.970 & 0.971 & 0.974 & 0.985\end{array}$

$\begin{array}{lllll}0.976 & 0.977 & 0.979 & 0.981 & 0.991\end{array}$

$\begin{array}{lllll}0.980 & 0.980 & 0.982 & 0.983 & 0.990\end{array}$

$\begin{array}{llllll}0.999 & 0.998 & 0.999 & 1.000 & 1.001 & 1.009\end{array}$

$\begin{array}{llllll}1.058 & 1.059 & 1.060 & 1.062 & 1.064 & 1.073\end{array}$

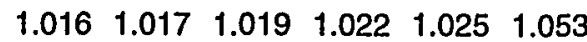

$\begin{array}{lllllll}1.003 & 1.004 & 1.005 & 1.008 & 1.012 & 1.017 & 1.048\end{array}$

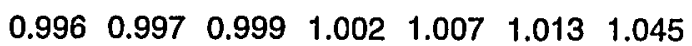

$\begin{array}{llllllll}0.990 & 0.991 & 0.992 & 0.995 & 0.999 & 1.004 & 1.011 & 1.043\end{array}$

$\begin{array}{llllllll}0.988 & 0.988 & 0.990 & 0.993 & 0.997 & 1.003 & 1.011 & 1.043\end{array}$

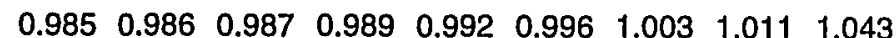

$\begin{array}{lllllllll}0.985 & 0.985 & 0.986 & 0.988 & 0.992 & 0.996 & 1.003 & 1.011 & 1.044\end{array}$

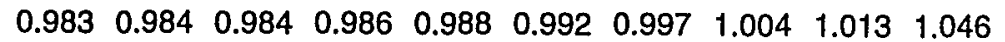

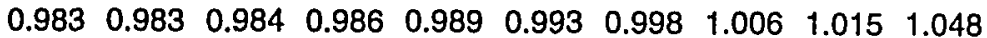

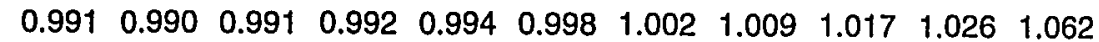

Fig. 4.43. Relative fission rate distribution for Variant 13, State 4, at $50 \mathrm{MWd} / \mathrm{kg}$. 
0.989

0.977

$0.970 \quad 0.979$

$0.9730 .982^{\circ}$

$\begin{array}{lll}0.975 & 0.976 & 0.985\end{array}$

$\begin{array}{lll}0.979 & 0.980 & 0.989\end{array}$

$\begin{array}{llll}0.982 & 0.983 & 0.984 & 0.994\end{array}$

$\begin{array}{llll}0.987 & 0.988 & 0.989 & 0.998\end{array}$

$\begin{array}{lllll}0.989 & 0.990 & 0.991 & 0.992 & 1.000\end{array}$

$\begin{array}{lllll}0.990 & 0.990 & 0.990 & 0.991 & 0.997\end{array}$

$\begin{array}{llllll}1.009 & 1.009 & 1.009 & 1.010 & 1.010 & 1.019\end{array}$

$\begin{array}{llllll}1.055 & 1.056 & 1.056 & 1.057 & 1.056 & 1.065\end{array}$

$\begin{array}{llllll}1.014 & 1.014 & 1.015 & 1.016 & 1.016 & 1.044\end{array}$

$\begin{array}{lllllll}1.001 & 1.002 & 1.003 & 1.004 & 1.007 & 1.009 & 1.039\end{array}$

$\begin{array}{lllllll}0.994 & 0.995 & 0.996 & 0.998 & 1.001 & 1.005 & 1.036\end{array}$

$\begin{array}{llllllll}0.988 & 0.989 & 0.990 & 0.992 & 0.995 & 0.998 & 1.003 & 1.034\end{array}$

$\begin{array}{llllllll}0.986 & 0.987 & 0.988 & 0.990 & 0.993 & 0.997 & 1.001 & 1.033\end{array}$

$\begin{array}{lllllllll}0.983 & 0.984 & 0.985 & 0.986 & 0.988 & 0.992 & 0.996 & 1.001 & 1.033\end{array}$

$\begin{array}{llllllllll}0.983 & 0.983 & 0.984 & 0.986 & 0.988 & 0.991 & 0.996 & 1.001 & 1.033\end{array}$

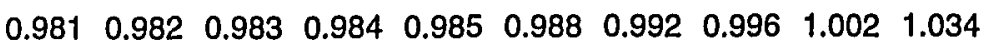

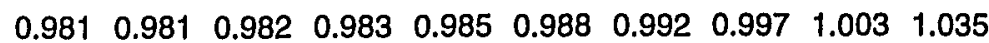

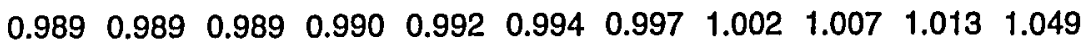

Fig. 4.44. Relative fission rate distribution for Variant 13, State 4 , at $60 \mathrm{MWd} / \mathrm{kg}$.
0.887

0.872

$0.860 \quad 0.870$

$0.868 \quad 0.876$

$\begin{array}{lll}0.870 & 0.871 & 0.883\end{array}$

$\begin{array}{lll}0.884 & 0.883 & 0.899\end{array}$

$\begin{array}{llll}0.901 & 0.902 & 0.907 & 0.932\end{array}$

$\begin{array}{llll}0.951 & 0.952 & 0.962 & 1.006\end{array}$

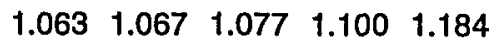

$\begin{array}{lllll}0.861 & 0.868 & 0.879 & 0.905 & 0.985\end{array}$

$\begin{array}{llllll}0.933 & 0.939 & 0.948 & 0.963 & 0.998 & 1.079\end{array}$

$\begin{array}{llllll}0.990 & 0.991 & 0.997 & 1.010 & 1.037 & 1.085\end{array}$

$\begin{array}{llllll}0.985 & 0.987 & 0.995 & 1.010 & 1.039 & 1.101\end{array}$

$\begin{array}{lllllll}0.983 & 0.985 & 0.989 & 0.998 & 1.014 & 1.047 & 1.108\end{array}$

$\begin{array}{llllllll}0.986 & 0.987 & 0.992 & 1.001 & 1.018 & 1.051 & 1.111\end{array}$

$\begin{array}{llllllll}0.984 & 0.985 & 0.988 & 0.993 & 1.003 & 1.021 & 1.054 & 1.113\end{array}$

$\begin{array}{llllllll}0.985 & 0.986 & 0.989 & 0.994 & 1.005 & 1.023 & 1.056 & 1.115\end{array}$

$\begin{array}{lllllllll}0.983 & 0.984 & 0.986 & 0.989 & 0.995 & 1.006 & 1.024 & 1.057 & 1.118\end{array}$

$\begin{array}{lllllllll}0.984 & 0.984 & 0.986 & 0.990 & 0.996 & 1.007 & 1.026 & 1.060 & 1.121\end{array}$

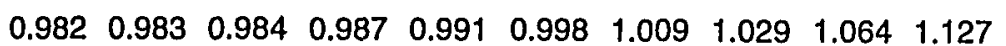

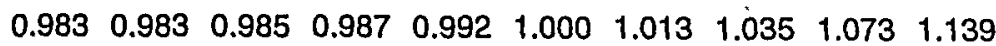

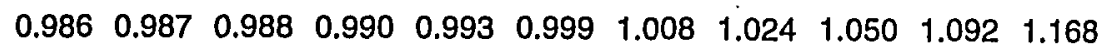

Fig. 4.45. Relative fission rate distribution for Variant 14, State 1 , at $0 \mathrm{MWd} / \mathrm{kg}$. 


\subsection{1}

0.887

0.8760 .885

0.8840 .892

$\begin{array}{lll}0.887 & 0.887 & 0.899\end{array}$

$\begin{array}{lll}0.901 & 0.900 & 0.915\end{array}$

$\begin{array}{llll}0.918 & 0.919 & 0.925 & 0.949\end{array}$

$\begin{array}{llll}0.966 & 0.967 & 0.978 & 1.018\end{array}$

$\begin{array}{lllll}1.060 & 1.063 & 1.073 & 1.094 & 1.160\end{array}$

$\begin{array}{lllll}0.835 & 0.839 & 0.848 & 0.867 & 0.915\end{array}$

$\begin{array}{lllllll}0.849 & 0.852 & 0.858 & 0.867 & 0.886 & 0.924\end{array}$

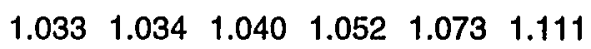

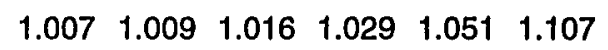

$\begin{array}{lllllll}0.996 & 0.998 & 1.002 & 1.009 & 1.023 & 1.051 & 1.106\end{array}$

$\begin{array}{lllllll}0.993 & 0.994 & 0.999 & 1.007 & 1.022 & 1.051 & 1.107\end{array}$

$\begin{array}{llllllll}0.989 & 0.990 & 0.993 & 0.998 & 1.007 & 1.023 & 1.051 & 1.108\end{array}$

$\begin{array}{llllllll}0.989 & 0.989 & 0.992 & 0.998 & 1.007 & 1.024 & 1.053 & 1.109\end{array}$

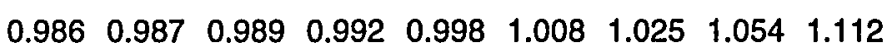

$\begin{array}{lllllllll}0.988 & 0.988 & 0.990 & 0.993 & 0.999 & 1.009 & 1.027 & 1.057 & 1.115\end{array}$

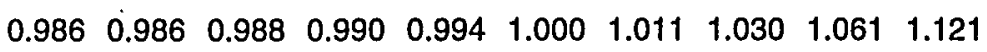

$\begin{array}{llllllllll}0.987 & 0.987 & 0.988 & 0.991 & 0.995 & 1.002 & 1.015 & 1.035 & 1.069 & 1.130\end{array}$

$\begin{array}{lllllllllll}0.991 & 0.991 & 0.992 & 0.994 & 0.997 & 1.003 & 1.012 & 1.027 & 1.051 & 1.088 & 1.158\end{array}$

Fig. 4.46. Relative fission rate distribution for Variant 14, State 1 , at $10 \mathrm{MWd} / \mathrm{kg}$.
0.939

0.926

0.9160 .924

0.9230 .931

$\begin{array}{lll}0.926 & 0.927 & 0.939\end{array}$

0.9410 .9410 .956

$\begin{array}{llll}0.959 & 0.960 & 0.966 & 0.989\end{array}$

1.0021 .0041 .0151 .050

$\begin{array}{llll}1.073 & 1.076 \quad 1.085 & 1.102 & 1.147\end{array}$

$\begin{array}{lllll}0.837 & 0.840 & 0.846 & 0.859 & 0.885\end{array}$

$\begin{array}{llllll}0.830 & 0.832 & 0.835 & 0.842 & 0.852 & 0.873\end{array}$

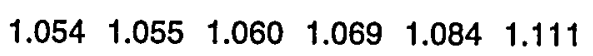

$\begin{array}{llllll}1.017 & 1.018 & 1.024 & 1.034 & 1.050 & 1.096\end{array}$

$\begin{array}{lllllll}1.000 & 1.001 & 1.005 & 1.011 & 1.023 & 1.044 & 1.091\end{array}$

$\begin{array}{lllllll}0.994 & 0.994 & 0.998 & 1.006 & 1.018 & 1.041 & 1.089\end{array}$

$\begin{array}{llllllll}0.987 & 0.988 & 0.991 & 0.995 & 1.003 & 1.017 & 1.040 & 1.088\end{array}$

$\begin{array}{lllllllll}0.986 & 0.986 & 0.989 & 0.994 & 1.002 & 1.016 & 1.040 & 1.089\end{array}$

$\begin{array}{lllllllll}0.983 & 0.983 & 0.985 & 0.988 & 0.993 & 1.002 & 1.017 & 1.041 & 1.090\end{array}$

$\begin{array}{lllllllll}0.984 & 0.983 & 0.985 & 0.988 & 0.994 & 1.003 & 1.018 & 1.043 & 1.093\end{array}$

$\begin{array}{llllllllll}0.981 & 0.981 & 0.983 & 0.985 & 0.989 & 0.995 & 1.004 & 1.020 & 1.046 & 1.097\end{array}$

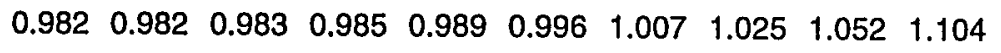

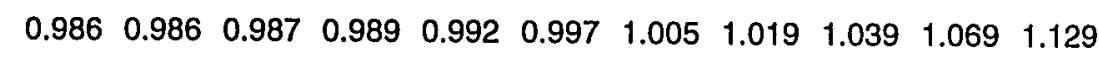

Fig. 4.47. Relative fission rate distribution for Variant 14, State 1, at $20 \mathrm{MWd} / \mathrm{kg}$. 


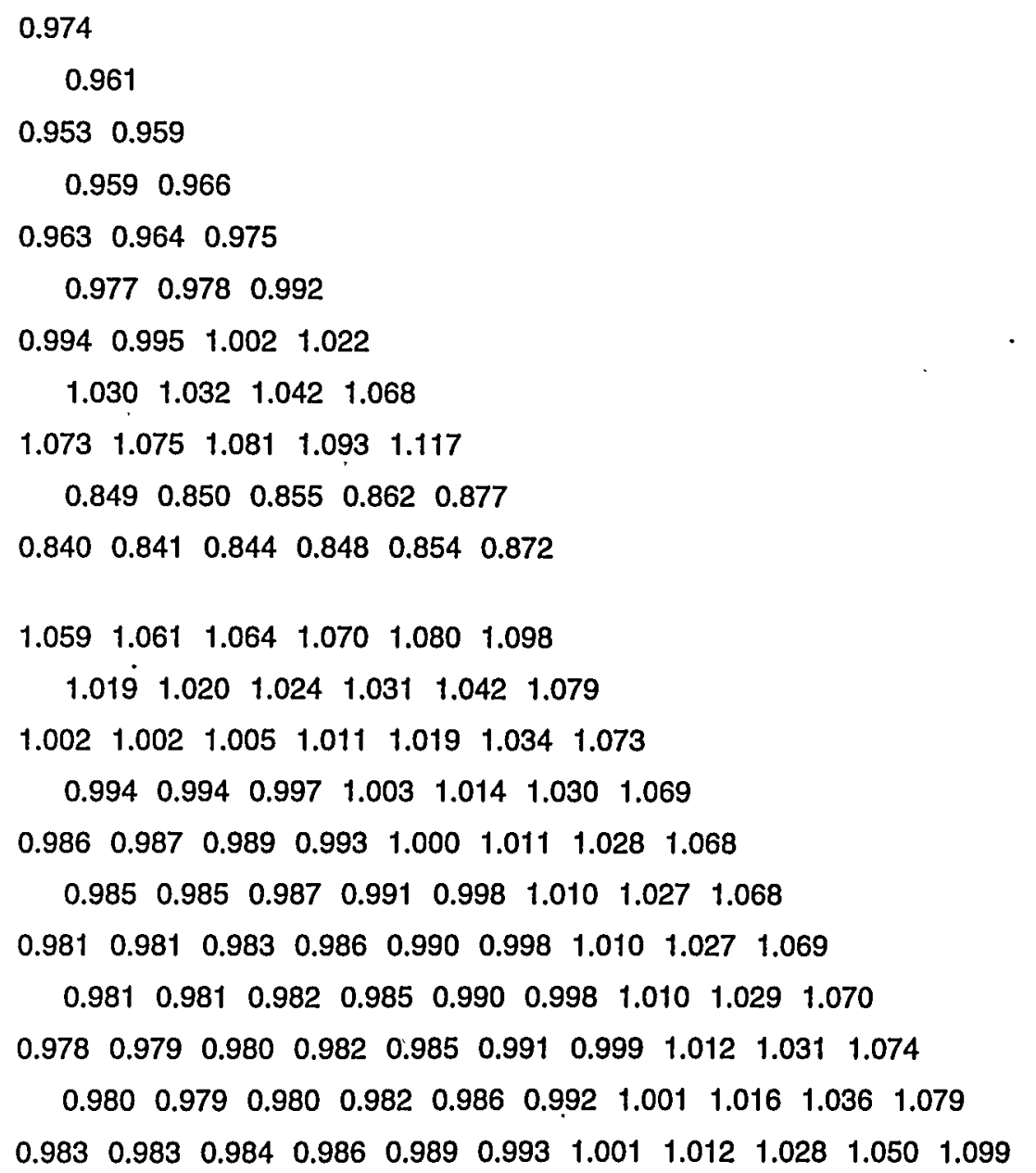

Fig. 4.48. Relative fission rate distribution for Variant 14, State 1 , at $30 \mathrm{MWd} / \mathrm{kg}$.

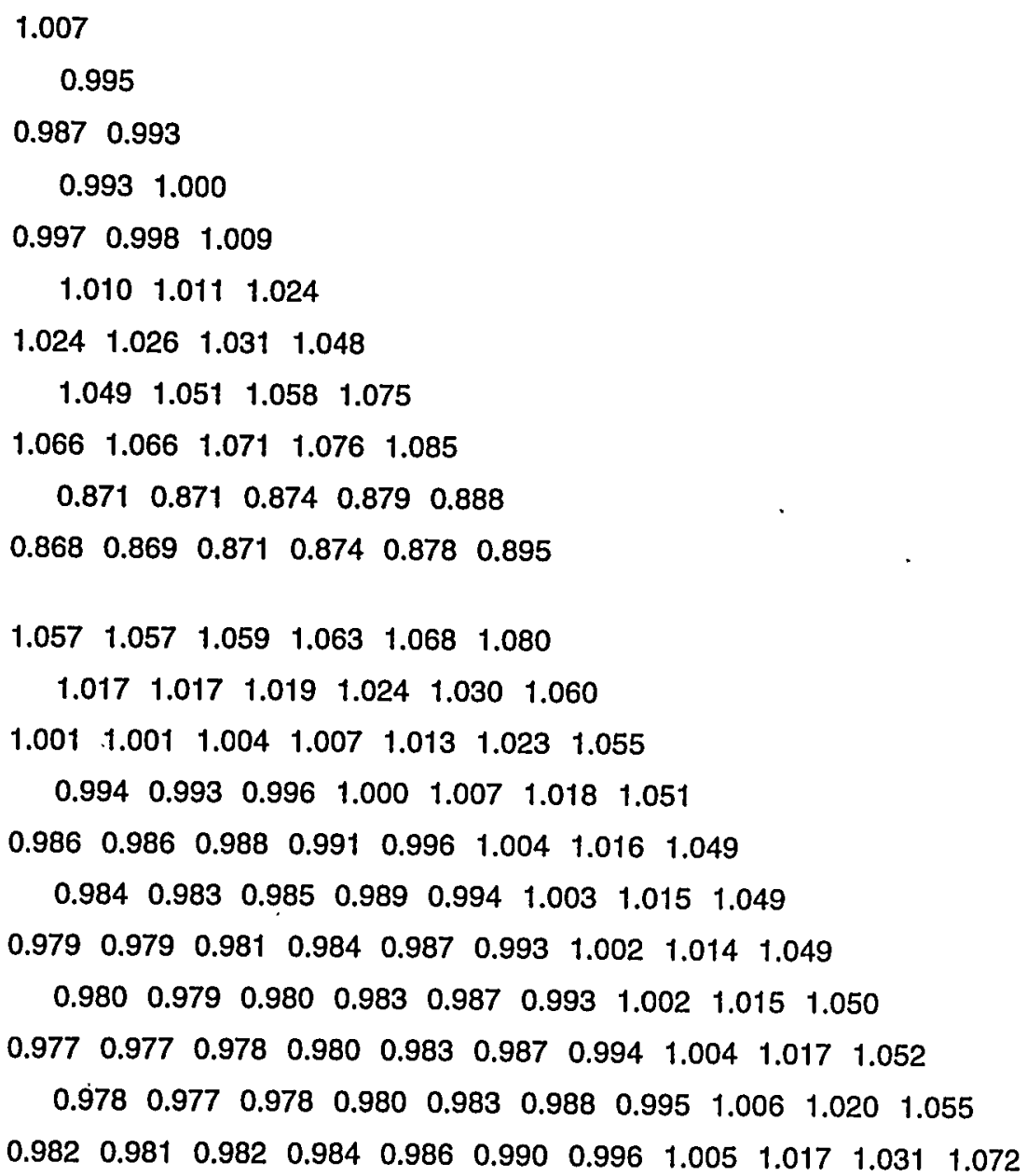

Fig. 4.49. Relative fission rate distribution for Variant 14, State 1, at $40 \mathrm{MWd} / \mathrm{kg}$. 


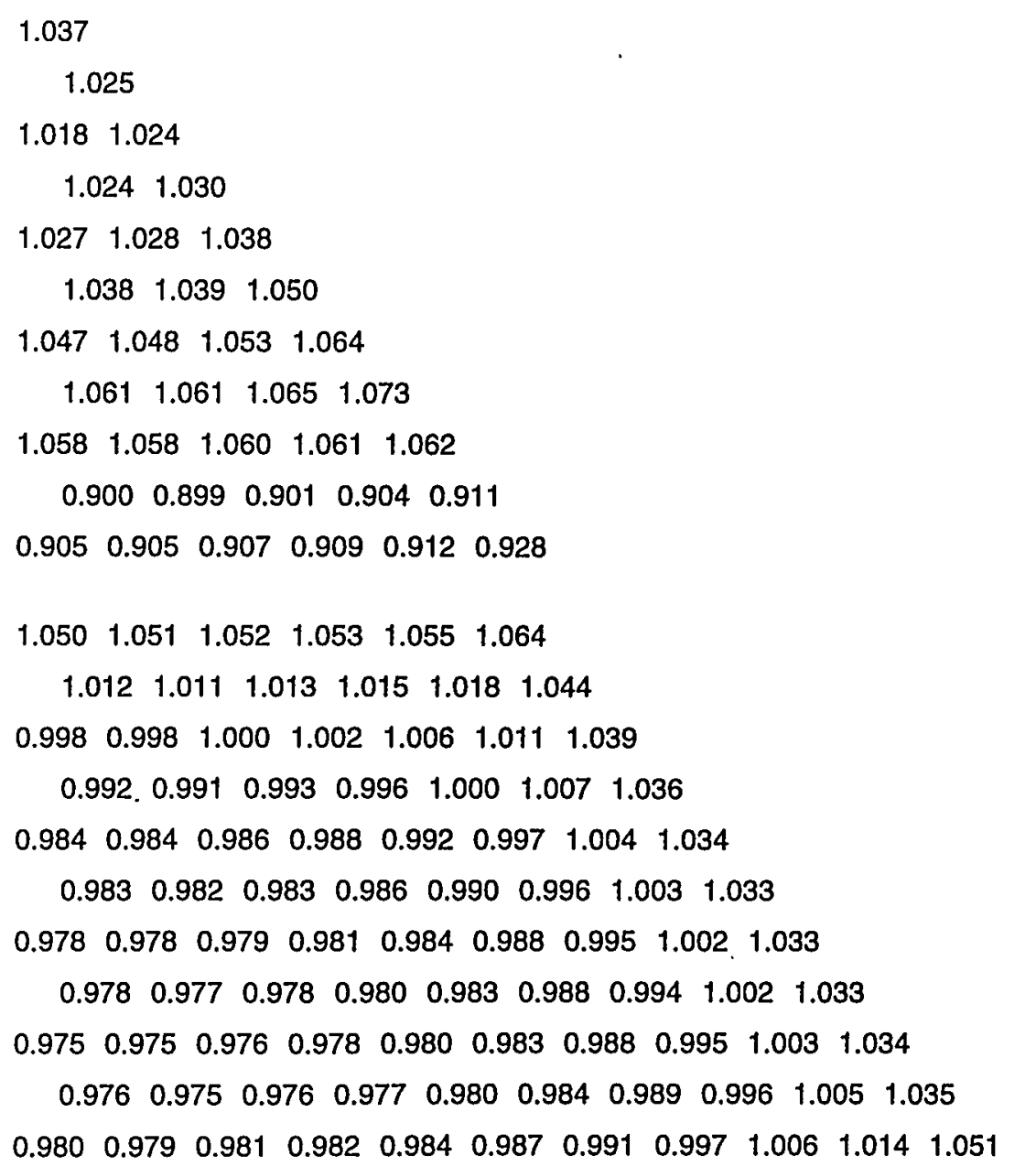

1.061

1.049

1.0421 .048

1.0471 .053

1.0501 .0501 .059

$\begin{array}{lll}1.058 & 1.058 & 1.066\end{array}$

$\begin{array}{llll}1.061 & 1.062 & 1.064 & 1.072\end{array}$

$\begin{array}{llll}1.065 & 1.064 & 1.065 & 1.068\end{array}$

$\begin{array}{lllll}1.051 & 1.051 & 1.051 & 1.050 & 1.048\end{array}$

$\begin{array}{lllll}0.930 & 0.929 & 0.930 & 0.932 & 0.938\end{array}$

$\begin{array}{lllllll}0.942 & 0.942 & 0.943 & 0.945 & 0.948 & 0.965\end{array}$

$\begin{array}{llllll}1.045 & 1.045 \quad 1.045 \quad 1.045 \quad 1.045 \quad 1.053\end{array}$

$\begin{array}{llllll}1.007 & 1.005 & 1.006 & 1.007 & 1.008 & 1.034\end{array}$

$\begin{array}{lllllll}0.994 & 0.994 & 0.996 & 0.997 & 0.999 & 1.002 & 1.029\end{array}$

$\begin{array}{lllllll}0.989 & 0.988 & 0.989 & 0.991 & 0.994 & 0.998 & 1.026\end{array}$

$\begin{array}{llllllll}0.982 & 0.982 & 0.983 & 0.985 & 0.987 & 0.991 & 0.995 & 1.024\end{array}$

$\begin{array}{llllllll}0.981 & 0.979 & 0.980 & 0.982 & 0.985 & 0.989 & 0.994 & 1.023\end{array}$

$\begin{array}{lllllllll}0.976 & 0.976 & 0.977 & 0.979 & 0.980 & 0.983 & 0.988 & 0.993 & 1.022\end{array}$

$\begin{array}{lllllllll}0.977 & 0.975 & 0.976 & 0.977 & 0.979 & 0.983 & 0.987 & 0.992 & 1.022\end{array}$

$\begin{array}{llllllllll}0.973 & 0.973 & 0.974 & 0.975 & 0.977 & 0.979 & 0.982 & 0.987 & 0.993 & 1.022\end{array}$

$\begin{array}{llllllllll}0.974 & 0.973 & 0.974 & 0.975 & 0.976 & 0.979 & 0.983 & 0.988 & 0.994 & 1.023\end{array}$.

$\begin{array}{lllllllllll}0.978 & 0.978 & 0.979 & 0.980 & 0.981 & 0.983 & 0.986 & 0.990 & 0.996 & 1.002 & 1.039\end{array}$
Fig. 4.50. Relative fission rate distribution for Variant 14, State 1 , at $50 \mathrm{MWd} / \mathrm{kg}$.
Fig. 4.51. Relative fission rate distribution for Variant 14, State 1 , at $60 \mathrm{MWd} / \mathrm{kg}$. 
0.891

0.881

0.8720 .879

0.8770 .883

$\begin{array}{llll}0.878 & 0.878 & 0.886\end{array}$

$\begin{array}{lll}0.886 & 0.885 & 0.895\end{array}$

$\begin{array}{llll}0.896 & 0.898 & 0.900 & 0.917\end{array}$

$\begin{array}{llll}0.935 & 0.935 & 0.942 & 0.973\end{array}$

$\begin{array}{lllll}1.035 & 1.038 & 1.043 & 1.059 & 1.127\end{array}$

$\begin{array}{llllll}0.832 & 0.835 & 0.841 & 0.861 & 0.926\end{array}$

$\begin{array}{lllllll}0.882 & 0.880 & 0.882 & 0.892 & 0.922 & 0.994\end{array}$

$\begin{array}{llllll}1.047 & 1.039 & 1.038 & 1.049 & 1.078 & 1.142\end{array}$

$\begin{array}{llllll}1.008 & 1.007 & 1.012 & 1.025 & 1.054 & 1.125\end{array}$ $\begin{array}{lllllll}0.996 & 0.997 & 0.999 & 1.005 & 1.018 & 1.047 & 1.112\end{array}$

$\begin{array}{llllllll}0.994 & 0.996 & 0.997 & 1.000 & 1.006 & 1.019 & 1.047 & 1.113\end{array}$

$\begin{array}{llllllll}0.996 & 0.996 & 0.998 & 1.000 & 1.007 & 1.020 & 1.048 & 1.114\end{array}$

$\begin{array}{lllllllll}0.996 & 0.997 & 0.997 & 0.999 & 1.002 & 1.008 & 1.021 & 1.050 & 1.116\end{array}$

$\begin{array}{lllllllll}0.998 & 0.998 & 0.998 & 1.000 & 1.003 & 1.009 & 1.022 & 1.052 & 1.119\end{array}$

$\begin{array}{llllllllll}0.997 & 0.998 & 0.998 & 0.999 & 1.001 & 1.004 & 1.010 & 1.024 & 1.055 & 1.125\end{array}$

$\begin{array}{llllllllll}0.998 & 0.999 & 0.999 & 1.000 & 1.001 & 1.005 & 1.012 & 1.028 & 1.063 & 1.139\end{array}$

$\begin{array}{lllllllllll}1.001 & 1.001 & 1.001 & 1.001 & 1.003 & 1.005 & 1.010 & 1.020 & 1.041 & 1.085 & 1.182\end{array}$

Fig. 4.52. Relative fission rate distribution for Variant 14, State 6, at $0 \mathrm{MWd} / \mathrm{kg}$.
0.913

0.904

0.8970 .903

0.9010 .906

$0.902 \quad 0.902 \quad 0.909$

$0.909 \quad 0.909 \quad 0.918$

$\begin{array}{llll}0.920 & 0.922 & 0.925 & 0.940\end{array}$

$\begin{array}{llll}0.957 & 0.957 & 0.964 & 0.993\end{array}$

$\begin{array}{lllll}1.040 & 1.042 & 1.047 \quad 1.062 & 1.115\end{array}$

$\begin{array}{lllll}0.810 & 0.810 & 0.815 & 0.829 & 0.867\end{array}$

$\begin{array}{llllll}0.812 & 0.807 & 0.807 & 0.812 & 0.829 & 0.865\end{array}$

$\begin{array}{llllll}1.077 & 1.069 & 1.068 & 1.077 & 1.101 & 1.153\end{array}$

$\begin{array}{llllll}1.021 & 1.021 & 1.025 & 1.036 & 1.059 & 1.119\end{array}$

$\begin{array}{lllllll}1.004 & 1.005 & 1.007 & 1.012 & 1.023 & 1.048 & 1.105\end{array}$

$\begin{array}{lllllll}1.000 & 1.001 & 1.003 & 1.008 & 1.019 & 1.043 & 1.101\end{array}$

$\begin{array}{llllllll}0.998 & 0.999 & 1.000 & 1.002 & 1.008 & 1.019 & 1.043 & 1.101\end{array}$

$\begin{array}{llllllll}0.999 & 1.000 & 1.001 & 1.003 & 1.008 & 1.020 & 1.044 & 1.103\end{array}$

$\begin{array}{lllllllll}0.999 & 1.000 & 1.001 & 1.002 & 1.004 & 1.010 & 1.021 & 1.046 & 1.104\end{array}$

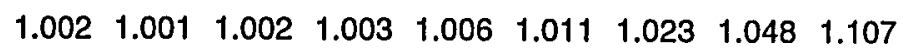

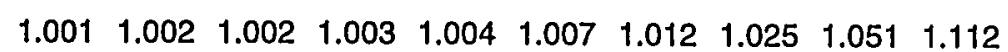

$\begin{array}{llllllllll}1.003 & 1.002 & 1.003 & 1.003 & 1.004 & 1.008 & 1.014 & 1.028 & 1.058 & 1.124\end{array}$

$\begin{array}{lllllllllll}1.005 & 1.005 & 1.005 & 1.006 & 1.006 & 1.008 & 1.012 & 1.021 & 1.040 & 1.078 & 1.164\end{array}$

Fig. 4.53. Relative fission rate distribution for Variant 14, State 6 , at $10 \mathrm{MWd} / \mathrm{kg}$. 
0.954

0.945

0.9390 .944

0.9420 .946

$\begin{array}{lll}0.942 & 0.943 \quad 0.949\end{array}$

$\begin{array}{lll}0.949 & 0.949 & 0.957\end{array}$

$\begin{array}{llll}0.959 & 0.961 & 0.964 & 0.977\end{array}$

$\begin{array}{llll}0.991 & 0.992 & 0.998 & 1.021\end{array}$

$\begin{array}{llllll}1.051 & 1.052 & 1.056 & 1.067 & 1.100\end{array}$

$\begin{array}{lllll}0.811 & 0.810 & 0.812 & 0.820 & 0.839\end{array}$

$\begin{array}{llllll}0.797 & 0.792 & 0.789 & 0.792 & 0.801 & 0.824\end{array}$

$\begin{array}{llllll}1.088 & 1.081 & 1.079 & 1.086 & 1.104 & 1.143\end{array}$

$\begin{array}{llllll}1.027 & 1.026 & 1.029 & 1.038 & 1.055 & 1.103\end{array}$ $\begin{array}{lllllll}1.027 & 1.026 & 1.029 & 1.038 & 1.055 & 1.103 \\ .007 & 1.008 & 1.009 & 1.013 & 1.022 & 1.040 & 1.086\end{array}$

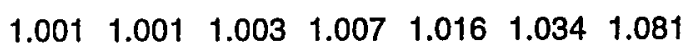

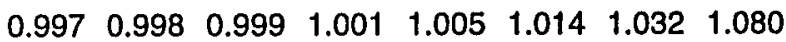

$\begin{array}{llllllll}0.998 & 0.998 & 0.999 & 1.001 & 1.005 & 1.014 & 1.032 & 1.080^{\circ}\end{array}$

$\begin{array}{llllllllll}0.998 & 0.998 & 0.999 & 1.000 & 1.001 & 1.006 & 1.015 & 1.033 & 1.081\end{array}$

$\begin{array}{lllllllll}1.000 & 0.999 & 1.000 & 1.000 & 1.002 & 1.006 & 1.016 & 1.035 & 1.083\end{array}$

$\begin{array}{llllllllll}0.999 & 1.000 & 1.000 & 1.000 & 1.001 & 1.003 & 1.007 & 1.017 & 1.037 & 1.088\end{array}$

$\begin{array}{llllllllll}1.001 & 1.000 & 1.000 & 1.000 & 1.001 & 1.003 & 1.008 & 1.020 & 1.043 & 1.097\end{array}$

$\begin{array}{lllllllllll}1.003 & 1.003 & 1.003 & 1.003 & 1.003 & 1.005 & 1.008 & 1.015 & 1.030 & 1.060 & 1.131\end{array}$

Fig. 4.54. Relative fission rate distribution for Variant 14, State 6, at $20 \mathrm{MWd} / \mathrm{kg}$.
0.997

0.989

0.9830 .987

0.9850 .988

$\begin{array}{lll}0.985 & 0.985 & 0.990\end{array}$

$\begin{array}{lll}0.990 & 0.990 & 0.997\end{array}$

$\begin{array}{llll}0.998 & 0.999 & 1.002 & 1.012\end{array}$

$\begin{array}{llll}1.021 & 1.021 & 1.026 & 1.041\end{array}$

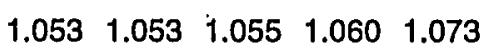

$\begin{array}{lllll}0.825 & 0.822 & 0.822 & 0.826 & 0.834\end{array}$

$\begin{array}{llllll}0.811 & 0.806 & 0.803 & 0.803 & 0.809 & 0.829\end{array}$

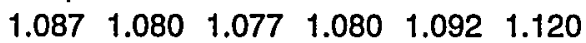

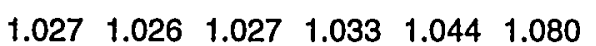

$\begin{array}{lllllll}1.007 & 1.008 & 1.009 & 1.011 & 1.017 & 1.028 & 1.064\end{array}$

$\begin{array}{lllllll}1.001 & 1.000 & 1.001 & 1.004 & 1.010 & 1.022 & 1.058\end{array}$

$\begin{array}{llllllll}0.997 & 0.997 & 0.998 & 0.999 & 1.001 & 1.007 & 1.019 & 1.056\end{array}$

$\begin{array}{llllllll}0.997 & 0.997 & 0.997 & 0.998 & 1.001 & 1.007 & 1.019 & 1.056\end{array}$

$\begin{array}{lllllllll}0.997 & 0.997 & 0.997 & 0.997 & 0.998 & 1.001 & 1.007 & 1.019 & 1.056\end{array}$

$\begin{array}{lllllllll}0.999 & 0.998 & 0.998 & 0.998 & 0.998 & 1.001 & 1.007 & 1.019 & 1.058\end{array}$

$\begin{array}{llllllllll}0.998 & 0.998 & 0.998 & 0.998 & 0.998 & 0.999 & 1.001 & 1.008 & 1.021 & 1.060\end{array}$

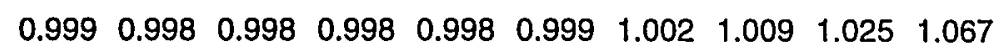

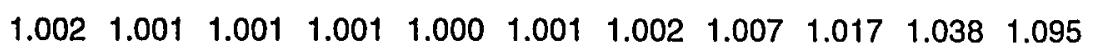

Fig. 4.55. Relative fission rate distribution for Variant 14, State 6 , at $30 \mathrm{MWd} / \mathrm{kg}$. 


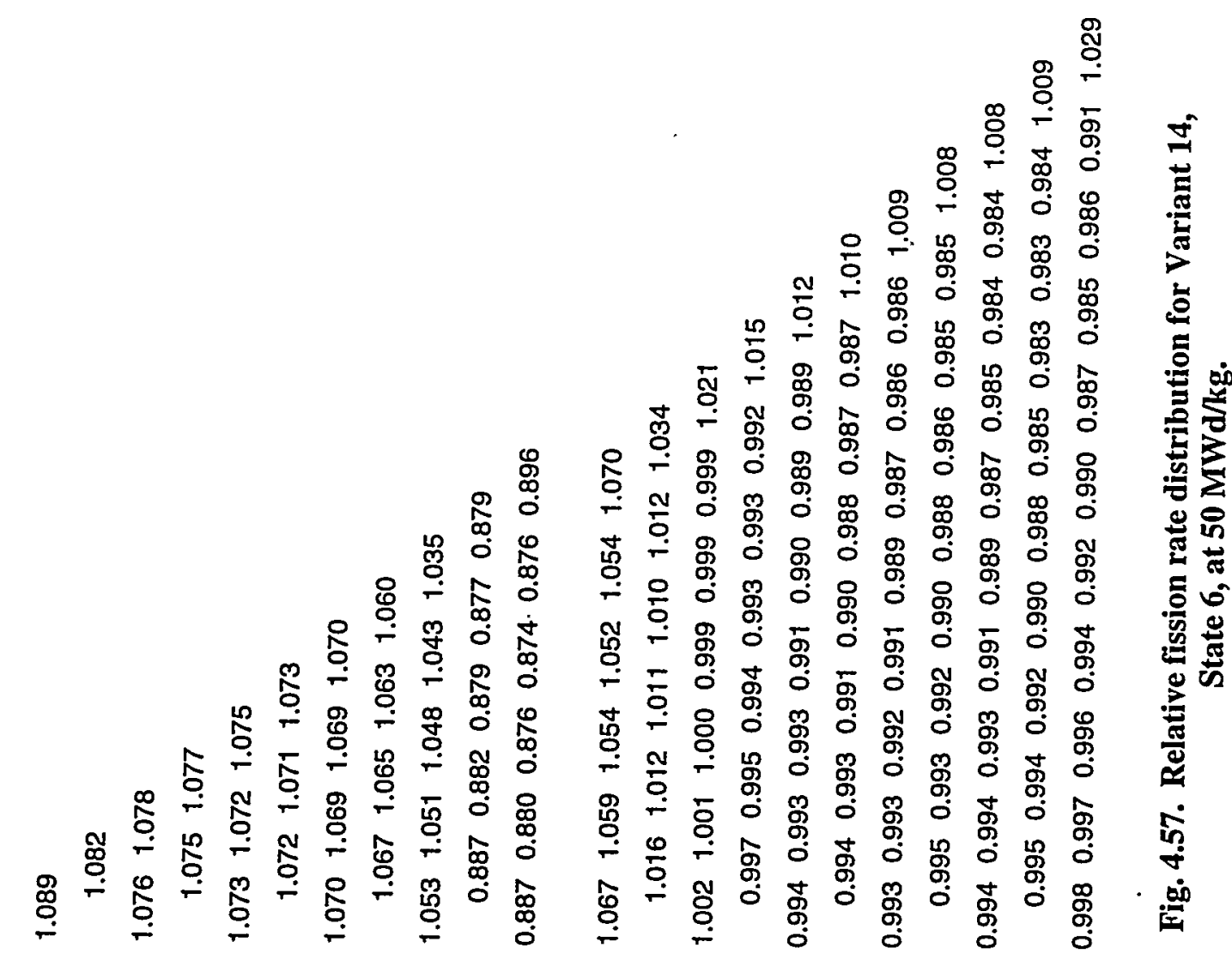

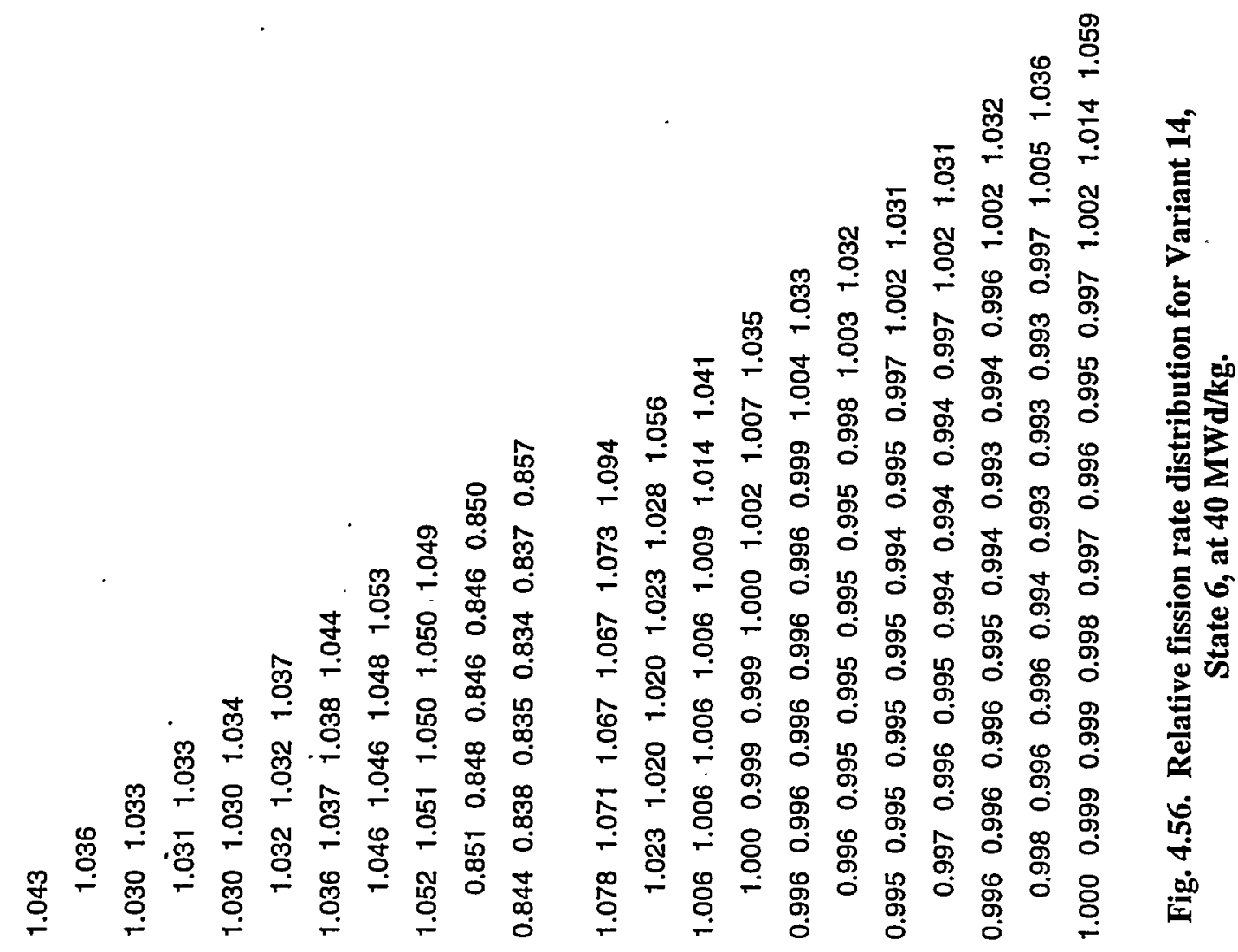


1.129

1.120

1.1141 .116

1.1121 .113

$\begin{array}{lll}1.107 & 1.107 & 1.107\end{array}$

$\begin{array}{lll}1.103 & 1.100 & 1.099\end{array}$

$\begin{array}{llll}1.094 & 1.093 & 1.090 & 1.087\end{array}$

$\begin{array}{llll}1.081 & 1.078 & 1.073 & 1.065\end{array}$

$\begin{array}{lllll}1.056 & 1.054 & 1.049 & 1.042 & 1.031\end{array}$

$\begin{array}{lllll}0.924 & 0.918 & 0.914 & 0.911 & 0.912\end{array}$

$\begin{array}{llllll}0.931 & 0.924 & 0.919 & 0.917 & 0.918 & 0.939\end{array}$

$\begin{array}{llllll}1.057 & 1.049 & 1.043 & 1.039 & 1.038 & 1.053\end{array}$

禺

$\begin{array}{llllll}1.008 & 1.004 & 1.001 & 0.999 & 0.998 & 1.019\end{array}$

$\begin{array}{lllllll}0.997 & 0.996 & 0.994 & 0.991 & 0.989 & 0.986 & 1.006\end{array}$

$\begin{array}{lllllll}0.993 & 0.991 & 0.989 & 0.986 & 0.983 & 0.980 & 1.000\end{array}$

$\begin{array}{llllllll}0.990 & 0.989 & 0.988 & 0.986 & 0.983 & 0.980 & 0.976 & 0.997\end{array}$

$\begin{array}{llllllll}0.991 & 0.989 & 0.987 & 0.984 & 0.981 & 0.977 & 0.974 & 0.995\end{array}$

$\begin{array}{lllllllll}0.990 & 0.989 & 0.989 & 0.986 & 0.983 & 0.979 & 0.975 & 0.972 & 0.993\end{array}$

$\begin{array}{lllllllll}0.992 & 0.990 & 0.988 & 0.985 & 0.982 & 0.978 & 0.974 & 0.970 & 0.991\end{array}$

$\begin{array}{llllllllll}0.991 & 0.990 & 0.989 & 0.987 & 0.984 & 0.980 & 0.976 & 0.971 & 0.968 & 0.990\end{array}$

$\begin{array}{llllllllll}0.992 & 0.990 & 0.988 & 0.986 & 0.982 & 0.978 & 0.973 & 0.969 & 0.967 & 0.990\end{array}$

$\begin{array}{lllllllllll}0.995 & 0.994 & 0.993 & 0.990 & 0.987 & 0.983 & 0.979 & 0.974 & 0.972 & 0.973 & 1.010\end{array}$

Fig. 4.58. Relative fission rate distribution for Variant 14, State 6 , at $60 \mathrm{MWd} / \mathrm{kg}$. 


\section{KENO CALCULATIONS}

An additional, preliminary analysis of Variant 14 was performed with the KENO-VI ${ }^{6}$ Monte Carlo code using cross sections from HELIOS calculations. HELIOS cross sections were used so that calculations could be performed at all desired burnups.

A computer code was written to transfer the cross-section data from a ZENITH output file into AMPX working libraries for State 1 at 0-, 10-, 20-, 30-, 40-, 50-, and 60-MWd/kg burnup. The preliminary analysis was based on the HELIOS 34-group cross-section libraries because of limits in editing cross sections using ZENITH. The memory requirements were too large for the 89- or 190-group libraries. Note that at the time these calculations were being performed, Scandpower was in the process of developing an official code for the transfer of cross-section data into AMPX working library format. This code, however, was not available for this analysis.

The KENO model includes an exact representation of the problem (pins in an array of hexagonal cells). The results of the calculations along with the HELIOS results are presented in Table 5.1. The differences between the HELIOS and KENO-VI results have not been completely investigated. The differences may be attributed to differences between the HELIOS and KENO-VI models of Variant 14, differences in the representation of the scattering approximation (KENO-VI uses a "pure" P1 approximation, while HELIOS uses a transport-corrected P0 approximation), or unidentified errors in the HELIOS-to-AMPX library conversion.

Table 5.1. Comparison of HELIOS and KENO-VI $k_{\text {eff }}$ results for Variant 14, State 1

\begin{tabular}{ccc}
\hline $\begin{array}{c}\text { Burnup } \\
\text { (MWd/kg) }\end{array}$ & \multicolumn{3}{c}{$\mathrm{k}_{\text {eff }}$} \\
\cline { 2 - 3 } & HELIOS & KENO-VI $(1 \sigma)$ \\
\hline & 1.0632 & $1.0494(0.0025)$ \\
20 & 0.9757 & $0.9612(0.0023)$ \\
30 & 0.9067 & $0.8932(0.0021)$ \\
40 & 0.8487 & $0.8376(0.0019)$ \\
50 & 0.7974 & $0.7848(0.0017)$ \\
60 & 0.7519 & $0.7422(0.0016)$ \\
\hline
\end{tabular}


Page Intentionally Blank 


\section{DISCUSSION AND CONCLUSIONS}

The series of VVER-1000 benchmark calculations were calculated with the HELIOS fuel assembly analysis code and the results documented. In calculating these benchmarks, we found the HELIOS system to be very flexible, allowing detailed modeling of complex systems.

In the preliminary analysis discussed in Chap. 3, it was shown that the 34-group library should be avoided for MOX systems based on a comparison of pin-cell $k_{\text {inf }}$ values with the results from the 190-group library. The 89-group library compares favorably with the 190-group library and is therefore recommended for the analysis of MOX systems. Note that adequate convergence with the 89- and 190-group libraries may require reducing the $\omega 3$ and $\omega 4$ relaxation parameters to 1.0.

The fission distributions from the multiassembly variants (V13 and V14) also show an unusual behavior in that there appears to be a slight peak in the fission rate in the pin at the center of the MOX assembly. Fig. 6.1 shows a radial traverse through the $\mathrm{MOX}$ and $\mathrm{UO}_{2}$ assemblies which demonstrates the peak at the central fuel pin for Variant 13 at $0 \mathrm{MWd} / \mathrm{kg}$. Based on the system's geometry and material compositions, the current, and hence the derivative of the neutron flux, should vanish at this central point (given the uniform composition in the center of the assembly), resulting in a flat fission density profile. Several other calculational tests were performed, and other slight anomalies were noted. These results were provided to Studsvik Scandpower, Inc., for further investigation.

These benchmarks have been analyzed with calculational systems used in Russia at the Russian Research Center, "Kurchatov Institute," in Moscow and at the Institute of Physics and Power Engineering in Obninsk. The calculational results are given in Ref. 1 for Variants 1-13. Preliminary comparisons of the multiplication factor results show good agreement with values typically within $0.2-0.5 \%$. The results of all of the calculations will be compared and will be the topic of a separate report. 


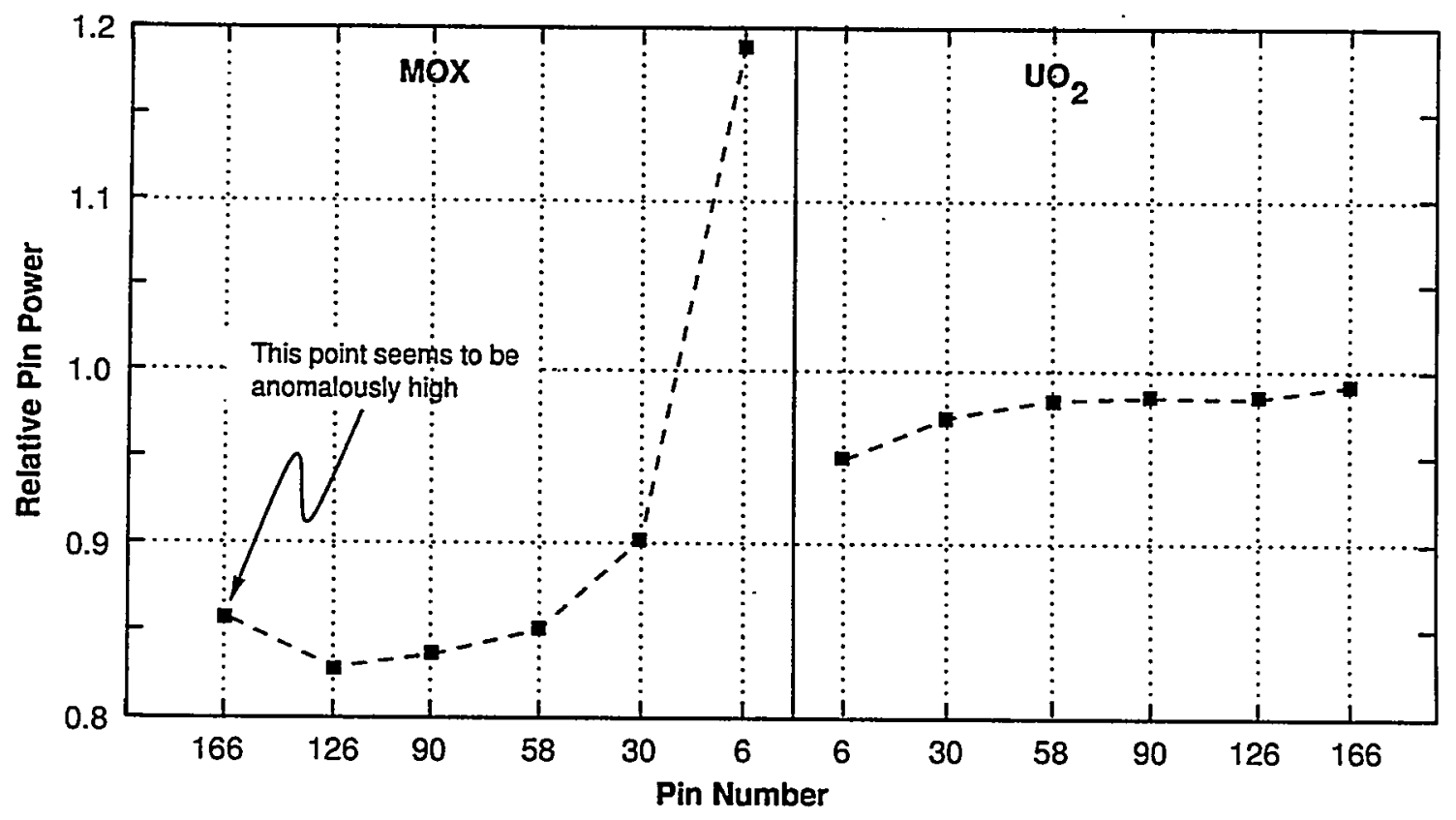

Fig. 6.1. Variant 14 relative pin power along assembly axis. 


\section{REFERENCES}

1. Neutronics Benchmarks for the Utilization of Mixed-Oxide Fuel: Joint U.S./Russian Progress Report for Fiscal Year 1997, Volume 3-Calculations Performed in Russia, ORNL/

TM-13603/V3, Lockheed Martin Energy Research Corp., Oak Ridge Natl. Lab., June 1998.

2. FMS-The Scandpower Fuel Management System, HELIOS Documentation, Scandpower, Inc., Dec. 1995.

3. J. J. Casal et al., "HELIOS: Geometric Capabilities of a New Fuel-Assembly Program," p. 10.2 1-1 in Proc. Int. Topl. Mtg. Advances in Mathematics, Computation, and Reactor Physics, Pittsburgh, Pennsylvania, April 28-May 2, 1991.

4. D. F. Hollenbach, L. M. Petrie, and N. F. Landers, "KENO-VI: A General Quadratic Version of the KENO Program," Vol. 2, Sect. F17, of SCALE, A Modular Code System for Performing Standardized Analyses for Licensing Evaluation, NUREG/CR-0200, Rev. 6 (ORNL/NUREG/CSD-2/V2/R6), September 1998.

5. A. P. Lazarenko, Russian Research Center, "Kurchatov Institute," personal communication, September 1997.

6. D. R. Vondy, The BOLD VENTURE Computation System for Nuclear Reactor Core Analysis, Version III, ORNL-5711, Union Carbide Corp., Nucl. Div., Oak Ridge Natl. Lab., June 1981. 
Page Intentionally Blank 


\section{ORNL/TM-1999/78}

\section{INTERNAL DISTRIBUTION}
1. B. B. Bevard
2. J. J. Carbajo
3. E. D. Collins
4. B. S. Cowell
5. M. D. DeHart
6. F. C. Difilippo
7. R. J. Ellis
8. M. B. Emmett
9-13. J. C. Gehin
14. S. R. Greene
15. T.W. Horning
16. D. T. Ingersoll

17. H. T. Kerr

18. R. A. Lillie

19. M. A. Kuliasha

20. G. E. Michaels

21. B. D. Murphy

22. D. L. Moses

23. C. V. Parks

24. R. T. Primm, III

25. C. C. Southmayd

26. Central Research Library

27-28. ORNL Laboratory Records (OSTT)

29. ORNL Laboratory Records-RC

\section{EXTERNAL DISTRIBUTION}

30. N. Abdurrahman, College of Engineering, Dept. of Mechanical Engineering, University of Texas, Austin, Texas 78712

31. M. L. Adams, Department of Nuclear Engineering, Texas A\&M University, Zachry 129, College Station, TX 77843

32. H. Akkurt, College of Engineering, Dept. of Mechanical Engineering, University of Texas, Austin, Texas 78712

33. D. Alberstein, Los Alamos National Laboratory, MS-E502, P.O. Box 1663, Los Alamos, NM 87545

34. J. Baker, Office of Fissile Materials Disposition, U.S. Department of Energy, MD-3, 1000 Independence Avenue SW, Washington, DC 20585

35. J. B. Briggs, Idaho National Environmental and Engineering Laboratory, P.O. Box 1625-3855, Idaho Falls, ID 83415-3855

36. L. Holgate, Office of Fissile Materials Disposition, U.S. Department of Energy, MD-1/2, 1000 Independence Avenue SW, Washington, DC 20585

37. A. Caponiti, Office of Fissile Materials Disposition, U.S. Department of Energy, MD-3, 1000 Independence Avenue SW, Washington, DC 20585

38. K. Chidester, Los Alamos National Laboratory, MS-E502, P.O. Box 1663, Los Alamos, NM 87545

39. W. Danker, U.S. Department of Energy, MD-3, 1000 Independence Avenue SW, Washington, DC 20585

40. C. Dourougie, 5 Allee Andre Malraux, 91330 Yerres, France

41. T. Gould, Lawrence Livermore National Laboratory, P.O. Box 808, MS-L186, Livermore, CA 94551

42. L. Jardine, Lawrence Livermore National Laboratory, P.O. Box 808, MS-L166, Livermore, CA 94551

43. Dr. A. Kalashnikov, Institute of Physics and Power Engineering, 1 Bondarenko Square, Obninsk, Kaluga Region, Russia 249020

44. D. E. Klein, Associate Vice Chancellor for Special Engineering Programs, The University of Texas System, 210 West Sixth Street, Austin, TX 78701 
45. J. O. Nulton, Office of Fissile Materials Disposition, U.S. Department of Energy, MD-3, 1000 Independence Avenue SW, Washington, DC 20585

46. S. L. Passman, Sandia National Laboratories, Suite 110, 950 L'Enfant Plaza S.W., Washington, DC 20024-2123

47. Dr. A. Pavlovitchev, Russian Research Center "Kurchatov Institute," Institute of Nuclear Reactors VVER Division, VVER Physics Department, 123182, Kurchatov Square, 1, Moscow, Russia

48. K. L. Peddicord, Associate Vice Chancellor, Texas A\&M University, 120 Zachry, College Station, TX 77843-3133

49. G. Radulescu, Framatom Cogema Fuels, 1261 Town Center Drive, MS-423, Las Vegas, Nevada 89143

50. W. D. Reece, Texas A\&M University, Department of Nuclear Engineering, Zachry 129, College Station, TX 77843-3133

51. P. T. Rhoads, Office of Fissile Materials Disposition, U.S. Department of Energy, MD-4, 1000 Independence Avenue SW, Washington, DC 20585

52. J. Thompson, Office of Fissile Materials Disposition, U.S. Department of Energy, MD-4, 1000 Independence Avenue SW, Washington, DC 20585

53. F. Trumble, Westinghouse Savannah River Company, Building 730R, Room 3402, WSRC, Aiken, SC 29808 